\title{
Rursus
}

Russus

Poiétique, réception et réécriture des textes antiques

$11 \mid 2017$

Nature et morale : sources, et postérité homilétique, des encyclopédies du XIIle siècle

\section{Le savoir astronomique et ses sources dans le De mundo et corporibus celestibus de Barthélemy l'Anglais}

Astronomical Knowledge and Its Sources in the De mundo et corporibus celestibus of Bartholomeus Anglicus

Isabelle Draelants et Eduard Frunzeanu

\section{(2)enEdition}

Journals

Édition électronique

URL : http://journals.openedition.org/rursus/1352

DOI : $10.4000 /$ rursus. 1352

ISSN : 1951-669X

Éditeur

Université Nice-Sophia Antipolis

Référence électronique

Isabelle Draelants et Eduard Frunzeanu, «Le savoir astronomique et ses sources dans le De mundo et corporibus celestibus de Barthélemy l'Anglais », Rursus [En ligne], 11 | 2017, mis en ligne le 22 décembre 2017, consulté le 03 mai 2019. URL : http://journals.openedition.org/rursus/1352 ; DOI : $10.4000 /$ rursus. 1352

Ce document a été généré automatiquement le 3 mai 2019.

Rursus 


\title{
Le savoir astronomique et ses sources dans le De mundo et corporibus celestibus de Barthélemy l'Anglais ${ }^{1}$
}

\author{
Astronomical Knowledge and Its Sources in the De mundo et corporibus \\ celestibus of Bartholomeus Anglicus
}

Isabelle Draelants et Eduard Frunzeanu

\section{NOTE DE L'AUTEUR}

Bibliographie. Les extraits du livre VIII, du De proprietatibus rerum, cités dans cet article, sont tirés de l'édition critique qui paraîtra sous peu chez Brepols. Elle a été réalisée d'après les manuscrits suivants, choisis par les coordinateurs de l'édition, Chr. MeierStaubah, H. Meyer, B. Van den Abeele : A : Paris, Bibl. nationale de France, lat. 16098 ; B : Paris, B.n.F., lat. 16099 ; C : København, Kongelike Bibl., Gl. kgl. S. 213 ; D : Cambridge, Fitzwilliam Museum, CFM 15 (L022) ; E : Paris, Bibliothèque de la Sorbonne, 123 (L133).

\section{Introduction}

Dans la plupart des manuscrits, le livre VIII du De proprietatibus rerum porte le titre De mundo et corporibus celestibus ${ }^{2}$. En son début, une brève description sémantique d'inspiration platonicienne met en évidence une des structures organisatrices de la compilation élaborée par Barthélemy l'Anglais ${ }^{3}$. Il s'agit des trois sens du terme mundus : l'archétype, le monde sensible et l'homme-microcosme, associés dans une relation transitive qui pose le premier monde en modèle du deuxième et le troisième comme l'image du précédent. Un critère temporel vient les hiérachiser : le premier est éternel, le 
deuxième perpétuel, le troisième est sous certains aspects perpétuel, sous d'autres, éphémère ${ }^{4}$ :

Le premier monde est éternel et demeure éternellement dans l'esprit divin.

Le second monde est perpétuel, tirant par la volonté divine son être perpétuel et son origine de rien.

Le troisième est quelque chose d'à la fois perpétuel et caduque, portant en lui la ressemblance de toutes choses.

Ce passage ressemble d'assez près à un extrait de la Philosophia mundi de Daniel de Morley, lui-même inspiré de l'Asclepius hermétique ${ }^{5}$ :

primus mundus fuit in mente, secundus in opere, tercius in imitatione.

Primus nec visibilis nec corporeus est, sed eternus,

secundus corporeus, visibilis, nec in hoc statu eternus,

tertius qui dicitur microchosmus, corporeus visibilis, sed in parte eternus.

Primus mundus est in eternitate figuratus, secundus cum tempore creatus, tercius in tempore formatus ${ }^{6}$

Au monde archétypal et à l'homme microcosme, Barthélemy dit avoir consacré les livres précédents, de I à VII, laissant entendre qu'il entame un nouveau volet réservé au monde sensible et au macrocosme. À ce sujet, dit-il, aliquid dicendum est, façon bien modeste de caractériser le contenu des douze livres suivants, de VIII à XIX, qui portent grosso modo sur le ciel et les réalités corrélatives à chacun des quatres éléments (feu, air, eau et terre).

Dans la lignée d'une tradition exégétique bien établie depuis l'apôtre Paul, et relayée par le souhait exprimé par Augustin dans le De doctrina christiana ${ }^{7}$, le prologue du De mundo annonce que l'exposé des propriétés des res du monde sensible a pour finalité la compréhension du sens spirituel et mystique de l'Écriture sainte, qui, comme le sens tropologique, n'est toutefois jamais abordé dans le texte-même du DPR. Ces significations trouveront place dans les notes marginales qui accompagnent le texte dans de nombreux manuscrits et qui mettent en valeur les correspondances entre l'ici-bas et l'au-delà que recelait, aux yeux des glossateurs, le texte de l'encyclopédiste.

Après avoir terminé avec l'aide de Dieu le traité sur les noms divins, les propriétés des anges, et aussi [celui] sur l'homme, sur les membres et sur les conditions accidentelles de celui-ci, il nous reste à nous occuper, autant qu'il nous sera possible, des propriétés du monde sensible, afin de pouvoir tirer des propriétés des opérations du Createur la matière de la louange divine. En effet, comme dit l'apôtre, 'les attributs invisibles de Dieu se laissent voir à l'intelligence à travers les choses créées' (Rom. 1.20). Et c'est pourquoi nous proposons d'intégrer dans cet opuscule quelques propriétés de ce monde et de ses contenus, sous la forme d'un bref compendium, pour que, par la similitude des propriétés des choses corporelles, nous puissions comprendre plus facilement le sens spirituel et mystique dans les écritures divines. Il faut donc en premier lieu commencer par les propriétés du monde. ${ }^{8}$

5 Dans le préambule au livre IX qui ménage la transition des propriétés du ciel à leurs effets, Barthélemy précise qu'il a traité précédemment des propriétés et parties célestes dont l'Écriture fait mention: Postquam autem diximus de proprietatibus celi et eius partibus de quibus sacra pagina facit mentionem. L'évocation de la finalité allégorique reste une justification nécessaire, mais - et c'est le cas de tous les compilateurs - la volonté de systématiser le plus possible les réalités créées et de mettre à profit la "bibliographie » compulsée va bien au-delà du périmètre exégétique: elle témoigne d'une véritable curiosité pour la nature. 


\section{Sources du livre VIII du De proprietatibus rerum}

6 S'agissant d'accompagner l'édition d'une compilation encyclopédique, notre commentaire s'intéresse au premier chef aux sources de celle-ci, car elles constituent la matière première du texte et peuvent seules livrer les informations sur le contexte intellectuel de la rédaction du texte.

\section{A. Aperçu général}

7 D'emblée, l'examen des sources du savoir astronomique de Barthélemy l'Anglais impose un constat décevant. En effet, les connaissances de l'encyclopédiste apparaissent peu approfondies, et ne sont le plus souvent pas à jour, malgré la présence de certaines informations puisées chez des philosophes naturalistes contemporains, elles-mêmes entachées d'incompréhensions. Les sources du De mundo, quand elles sont anciennes, sont rarement antiques, car elles datent de la période patristique - Basile de Césarée, Ambroise de Milan, Macrobe, Martianus Capella, Isidore de Séville, Bède le Vénérable. Puisées dans la tradition, elles sont le plus fréquemment transmises via un intermédiaire médiéval. Quand elles sont modernes et/ou contemporaines, ce qui est fréquent, elles ne sont pas avouées : Robert Grosseteste et d'autres philosophes contemporains se cachent sous des discussions anonymes relatives à la nature du ciel et à la lumière. Enfin, quand elles font état d'un savoir astrologique nourri de l'astrologie arabe, elles sont déguisées sous des noms difficiles à identifier comme celui de Misael, et proviennent de compilations dont on trouve des traces dans la tradition parallèle, véhiculée par Raymond de Marseille.

8 Ce constat d'un savoir peu spécialisé et transmis en partie de seconde main ne saurait surprendre, car il a été déjà souligné par les éditeurs d'autres livres du DPR. Il doit cependant être complété par une attention portée à la discrète mais incontestable nouveauté de certaines sources naturalistes, restées dissimulées jusqu'ici sous d'obscures références.

\section{Des marqueurs de source peu fiables, de nombreuses médiations insoupçonnées}

Dans la tradition patristique, Barthélemy privilégie l'autorité de Basile de Césarée et d'Ambroise de Milan qui ne paraissent ni l'un ni l'autre connus de première main. Ambroise n'est cité nommément qu'à trois reprises, la première à travers l'Hexaëmeron de Robert Grosseteste, tandis que les deux autres citations - on peut le supposer - circulaient déjà sous cette forme dans les multiples instruments lexicologiques disponibles. En effet, elles juxtaposent plusieurs groupes nominaux de l'Hexaëmeron d'Ambroise pour donner des définitions du Soleil et de la Lune aux sonorités versifiées 9

10 Le livre III des Étymologies d'Isidore, De mathematica, est peu utilisé. Lorsque c'est le cas, il s'agit de notions élémentaires reformulées profondément et transmises par des intermédiaires variés, ce que trahit un vocabulaire technique différent et de trop rares reprises des explications étymologico-ontologiques d'Isidore. Qui plus est, les citations associées au marqueur de source ${ }^{10}$ "Isidore » dans le livre VIII ne se retrouvent qu'à quelques exceptions dans la source alléguée, contrairement à ce qu'on peut remarquer dans d'autres livres du DPR pour les références dotées de renvois explicites à tel ou tel 
livre des Étymologies. D'un livre à l'autre, Barthélemy ne suit pas les mêmes «normes bibliographiques »; en outre, là où la référence est incomplète, la citation ne correspond pas au texte d'Isidore. Dès lors, on peut supposer qu'un intermédiaire a servi de filtre. En l'occurrence, ce pourrait être Guillaume de Conches et sa Philosophia mundi, que l'encyclopédiste contemporain de Barthélemy, Thomas de Cantimpré, utilise abondamment aussi dans le domaine astronomique. Ainsi la définition de l'horizon (Unde et circulus, ubi finitur, orizon, id est visus terminatio, nuncupatur, ut dicit Isidorus, DPR, VIII.6, 1. 44-46) se trouve presque dans les mêmes termes dans la Philosophia (II.7.19), alors qu'Isidore n'en fait mention nulle part. Tout le chapitre consacré aux cercles célestes est également mis sous l'autorité d'Isidore (hucusque Isidorus, DPR, VIII.7, 1.37). Lorsqu'il aborde ces notions dans le De natura rerum aussi bien que dans les livres III.44 et XIII.6 de ses Étymologies, Isidore reprend à ce sujet presque à la lettre les définitions données par Hygin dans son De astronomia; or chez Barthélemy, le texte compilé sous le nom d'Isidore est largement redevable à Macrobe et à Martianus Capella. L'imbrication de ces deux autorités pour traiter des cercles célestes est présente dans la Philosophia de Guillaume de Conches. Cependant, entre Guillaume et Barthélemy, un autre compilateur a dû ajouter quelques compléments à l'exposé. Cette compilation intermédiaire, dont nous discuterons plus bas, a été désignée par son éditrice comme l'Experimentator; c'est sa version que reprend de toute vraisemblance Barthélemy. Le nom de Guillaume de Conches, resté implicite sous le marqueur Isidorus à travers la compilation utilisée par Barthélemy, figure en revanche dans la table des auteurs qui accompagne bon nombre de manuscrits du DPR.

Ce type de filtrage caractérise également les citations mises sous le nom de Martianus Capella et de Bède, qui sont elles aussi puisées à la compilation dudit Experimentator. Pour ne donner qu'un exemple, la terminologie employée pour qualifier et décrire les phases de la Lune remonte en dernier lieu au livre VIII du De nuptiis Philologiae et Mercurii de Martianus Capella, dont l'exposé a probablement été décanté par l'entremise successive de Rémi d'Auxerre, de Guillaume de Conches et de l'Experimentator. A cet égard, il convient de rappeler que la compilation de Barthélemy voit le jour à un moment où le livre VIII du De nuptiis, consacré à l'astronomie, figure toujours dans les « introductions à la philosophie " produites en milieu universitaire ${ }^{11}$.

Les sources astronomiques du De mundo restent assez élémentaires et présentent une dominante astrologique; elles sont peu diversifiées et non spécialisées. Par exemple, Barthélemy recourt peu à l'œuvre de Ptolémée, qu'il ne connaît pas via ses grandes œuvres comme l'Almageste ou le Quadripartitum, et de même ne connaît Albumasar que d'une manière fragmentaire, via des intermédiaires récents ou des miscellanées astrologiques d'un niveau scientifique médiocre.

Quand ses sources sont modernes, elles sont victimes d'un usage parcimonieux et peu approfondi, souvent mal comprises, la plupart du temps sans que soient levées les contradictions théoriques qu'elles entraînent. La préséance que donne Barthélemy l'Anglais aux traités naturalistes ou d'astrologie en circulation au XII ${ }^{\mathrm{e}}$ siècle, comme la Philosophia de Guillaume de Conches ou l'Experimentator, plutôt qu'aux traités d'astronomie plus techniques récemment traduits comme ceux d'al-Battāni ${ }^{12}$ ou d'alBitrūji $1^{13}$, est le signe probable de la faible diffusion de ce type de littérature astronomique spécialisée dans le premier tiers du XIII ${ }^{e}$ siècle. L'enseignement, la compréhension et l'appropriation de ces traités augmentent en effet graduellement à partir de 1250. Même si, sous des graphies parfois hésitantes, les manuscrits du DPR comportent le nom 
d'Alfraganus (al-Farghāni), nous n'avons pu faire que deux rapprochements vagues entre les Differentie d'al-Farghāni ${ }^{14}$ et le De mundo.

Barthélemy n'a pas eu recours non plus à la Sphera de Jean de Sacrobosco qui, à partir de son époque et jusqu'au XVII ${ }^{e}$ siècle, devient l'introduction canonique au savoir astronomique. A fortiori, il n'utilise pas le commentaire de la Sphera attribué à Michel Scot. Il ne connait pas davantage la Theorica planetarum attribuée à Gérard de Crémone, qu'emploie pour sa part Michel Scot. Bien que le DPR présente des sources communes avec le Liber introductorius de Michel Scot (nous le montrerons plus loin), Barthélemy ne le cite pas, contrairement à ce qu'on aurait pourtant pu attendre en raison de la présence du nom de Michael Scotus dans la table des auteurs annexée à la fin de plusieurs manuscrits.

Dans l'ensemble, Barthélemy apparaît moins bien renseigné dans le domaine astrologique et astronomique que certains de ses contemporains comme Michel Scot, Guillaume d'Auvergne, Robert Grosseteste. Ce dernier, dont Barthélemy utilise l'Hexaëmeron et le De colore, connaît Ibn al-Haytham (Alhacen) et fait bon cas d'al-Bitrūji ${ }^{15}$, dont la théorie des sphères homocentriques est évoquée et confrontée au système de Ptolémée autant dans le De sphera que dans le De motu supercelestium ${ }^{16}$. Contemporains de Barthélemy, l'évêque parisien Guillaume d'Auvergne et le dominicain Albert le Grand connaissent, de première main ou par l'entremise d'abrégés, certaines thèses défendues par le De scientia stellarum d'al-Battāni et le De motibus celorum d'al-Bitrūji ${ }^{17}$.

$\mathrm{Vu}$ le petit nombre de références précises, les sources de Barthélemy apparaissent très rarement tirées d'originalia, mais le plus souvent issues de compilations, parfois récentes comme celles de l'Experimentator, ou encore d'Arnold de Saxe, comme on le verra cidessous. Elles pourraient aussi ne pas être seulement livresques. Lecteur sententiaire, il a en effet pu recourir à un arsenal de gloses peu connues aujourd'hui, parfois déjà empruntées par les auctoritates dont il se sert. Dès lors, il est difficile de rapporter tous les aspects qu'il a abordés à des œuvres connues, actuellement éditées. L'hypothèse d'un savoir partagé, issu de l'enseignement et des échanges contemporains, est soutenue par quelques indices, par exemple au sein de la section portant sur la lumière et la couleur. Pour certains textes, il est clair que Barthélemy l'Anglais a eu aussi accès à des commentaires, dont nous n'avons pas toujours réussi à retrouver l'origine. C'est le cas du Timée de Platon, comme en témoigne la section sur la matière primordiale où chacune des propriétés de la matière énumérées par Platon est reprise et commentée ${ }^{18}$. Il en va de même pour un extrait du De divinis nominibus du Pseudo-Denys l'Aréopagite, placé dans le chapitre De sole (VIII.17, 1. 58-195) ${ }^{19}$.

Habitant tous les esprits des prédicateurs, la hiérarchie des auctoritates, exposée entre autres dans le décret du Pseudo-Gélase ${ }^{20}$, imposait qu'on donnât plus de poids aux Pères de l'Eglise et aux auteurs anciens qu'aux recentiores, au point que la présence de ces derniers, effective, disparaissait derrière un jalonnement parcimonieux, et de moins en moins significatif, au profit d'autorités mieux reconnues. Les références aux sources utilisées sont donc très trompeuses pour la majorité d'entre elles et ne permettent pas, à partir des seuls "marqueurs", d'inférer une chronologie de la documentation ou de générer une liste d'auteurs et d'œuvres que Barthélemy aurait réellement eus à sa disposition. Une comparaison systématique et minutieuse des sources s'impose afin de déceler les différents faisceaux rassemblés par le compilateur. 


\section{Quid de la table des auteurs?}

18 Le rapide aperçu que nous venons de donner montre que le tableau réel de la documentation que Barthélemy a compulsée ne correspond que partiellement, d'une part, aux marqueurs de sources qui parsèment sa compilation et, d'autre part, à la liste des autorités insérée à la fin du livre XIX. Cette liste, qui ne comporte pas la même série de noms dans tous les manuscrits, est divisée, comme l'annonce le compilateur, entre sancti-theologi et philosophi, deux groupes qui réunissent les autorités que Barthélemy aurait citées en matière de théologie et de philosophie naturelle. Elle contient des noms qui ne sont pas mentionnés au fil du texte, comme ceux de Guillaume de Conches, Michel Scot, Robert Grosseteste (indexé sous Robertus Lincolnensis). Dans la tradition manuscrite telle qu'elle subsiste, la table et les marqueurs de sources ne concordent pas. Par exemple, au livre VIII, on voit apparaître à plusieurs reprises le marqueur Rabanus, accompagnant toujours des citations tirées en réalité de l'Hexaëmeron de Robert Grosseteste. Raban Maur quant à lui est bel et bien cité dans d'autres livres du DPR comme source directe ou par le biais de la glose ordinaire. Il y eut donc un travestissement ou une confusion très précoce dans les manuscrits de Barthélemy l'Anglais entre "Rab.» et " Rob. », confusion peutêtre déjà présente dans le texte de l'Experimentator qui a servi d'intermédiaire pour nombre d'extraits du livre VIII ${ }^{21}$.

Par ailleurs, la référence à un certain commentator au sujet d'une définition de l'axe du monde attribuée à Aristote ${ }^{22}$ invite à chercher du côté des commentaires d'Averroès, dont le nom est également présent dans la table. On sait que Robert Grosseteste est un des premiers à avoir utilisé les traductions latines d'Averroès. Les études de R. A. Gauthier ont montré que les commentaires d'Averroès à la Métaphysique et au De anima traduits par Michel Scot étaient connus à la Faculté des arts à Paris vers 1225. C'est à ce moment que Grosseteste aurait commencé à les découvrir, et plusieurs de ses écrits des années 1228-1235 en sont nourris ${ }^{23}$, ce qui n'est apparemment pas le cas de Barthélemy.

Il existe en revanche des noms que Barthélemy cite dans le corps du texte, mais qui n'apparaissent pas dans la table récapitulative - celle-ci, il est vrai, ne se veut pas exhaustive, le syntagme istorum et multorum aliorum laissant supposer une bibliographie plus large. Ainsi en est-il du contemporain franciscain Alexandre de Halès, dont l'apparition explicite peut être interprétée comme une signature franciscaine de la compilation. Les renvois à répétition dans le livre VIII à un certain Misael ainsi qu'à Messahalla, dont les graphies varient considérablement dans les manuscrits, laissent croire à l'existence de deux auteurs, alors que dans la liste finale ne figure qu'un Miselat astrologus. Des repérages que nous avons pu faire, il résulte que les passages associés à la forme Mischalath (Mischalach, Misalac) proviennent d'un traité de Messahalla, alors que le marqueur Misael (Misa, Mesael, Misali) est probablement une déformation du nom de Sahl ibn Bisr, dont nous examinons plus bas l'intermédiaire de transmission. 


\title{
B. Sources contemporaines directes, indirectes, inconnues ou implicites
}

\author{
Le problème de la tradition parallèle : l'exemple de la compilation appelée \\ Experimentator
}

21 Comme nous l'avons indiqué, plusieurs auteurs (Ambroise de Milan, Martianus Capella, Isidore de Séville, Bède, Robert Grosseteste) furent connus de Barthélemy via une compilation qui, comme d'autres, visait à présenter le Créateur et sa création. Il s'agit d'une œuvre anonyme associée au nom d'Experimentator depuis la thèse de Janine Deus, qui en a identifié deux versions longues et une abrégée ${ }^{24}$. Les recoupements entre cette compilation et les passages mis sous le nom d'un experimentator dans le Liber de natura rerum de Thomas de Cantimpré ont porté J. Deus à attribuer à cet auteur anonyme le recueil des proprietates rerum qu'elle a étudié. C'est aussi l'argument sur lequel elle a appuyé la datation supposée de la version originelle, perdue : entre 1220 et 1225/1226. Par ailleurs, dans ses diverses rédactions, ce texte comporte une structure similaire et des préambules qui sont identiques à l'encyclopédie naturelle de Barthélemy l'Anglais. C'est à partir de ces similitudes que Chr. Hünemörder et J. Deus ont soutenu l'hypothèse de l'utilisation de cette compilation par Barthélemy.

$22 \mathrm{~J}$. Deus voyait dans une version contenue dans quatre manuscrits la rédaction I de l' Experimentator ${ }^{25}$. Elle en a, en outre, isolé une deuxième version, conservée dans six manuscrits ${ }^{26}$, ainsi qu'une version abrégée de la version I, contenue dans un seul manuscrit ${ }^{27}$. Sans souscrire à la démonstration de J. Deus, H. Meyer et B. Van den Abeele ont considéré que le texte du soi-disant Experimentator, dans ses différentes formes, n'est autre que le témoin d'une révision en treize livres, largement remaniée, du De proprietatibus rerum de Barthélemy, la «13-Bücher-Fassung ${ }^{28}$. Reprenant plus récemment le dossier des relations entre l'Experimentator, Thomas de Cantimpré et Barthélemy l'Anglais, B. Van den Abeele ${ }^{29}$ arrive aux conclusions suivantes : 1) un texte de l'Experimentator a existé, mais ne subsiste plus, dans l'état qui a été connu par Thomas de Cantimpré ; ce serait la « version 0 »; 2) cette « version 0 » n'est pas le texte que J. Deus a identifié comme étant l'Experimentator; 3) ce que J. Deus appelle l'Experimentator est en fait la version abrégée en 13 livres et remaniée du DPR (qui connait les trois rédactions départagées par J. Deus dans sa thèse), texte dans lequel ont été insérés des passages tirés de la " version 0 ", présents chez Thomas de Cantimpré également. L'Experimentator édité par J. Deus se serait donc servi de Barthélemy l'Anglais et pas l'inverse, tout en exploitant une source commune avec Thomas de Cantimpré.

Il y aurait lieu d'affiner davantage les relations entre les différentes versions du texte de l' Experimentator, puisque le manuscrit de Stuttgart comporte, dans le livre consacré aux anges, un long extrait de la Hierarchia d'Alain de Lille ${ }^{30}$ qui n'est pas présent dans le manuscrit Berlin, Hamilton 277 que Deus range pourtant sous la même version que Stuttgart. Ce n'est toutefois pas ici le lieu de reprendre toutes ces questions d'embranchement. À la différence des comparaisons précédemment menées sur les livres consacrés aux mondes minéral, végétal et animal, nous allons focaliser notre discussion de la relation entre l'Experimentator et Barthélemy l'Anglais sur la section des réalités célestes, afin de montrer qu'il existe des éléments susceptibles de conforter l'hypothèse, avancée déjà par Deus, que Barthélemy a utilisé le texte de l'Experimentator et non pas l'inverse, comme le soutiennent $\mathrm{H}$. Meyer et B. Van den Abeele. 
24 Pour constituer sa compilation, l'Experimentator a repris à plusieurs endroits des gloses et des commentaires qui accompagnaient différents textes classiques et patristiques. Dans le livre sur les anges par exemple, les œuvres de Pseudo-Denys l'Aréopagite sont souvent appropriées par l'entremise d'Hugues de Saint-Victor. Chaque fois qu'une même citation apparaît autant chez Barthélemy que chez l'Experimentator, on constate chez ce dernier une plus grande fidélité envers le texte-source. Dans l'exemple suivant, on pourra remarquer que Barthélemy perd une phrase en cours de route (que nous avons soulignée), condense la syntaxe et change quelques termes (que nous avons mis en gras) :

\begin{tabular}{|c|c|c|}
\hline $\begin{array}{l}\text { Experimentator, } \\
\text { Stuttgart, Württembergische } \\
\text { Landesbibliothek, med. et phys. } \\
2^{\circ} 24, \text { f. } 9 \mathrm{~V}\end{array}$ & $\begin{array}{l}\text { Barthélemy l'Anglais, } \\
\text { DPR, I, cap. 7, p. 114, } 1 . \\
60-73\end{array}$ & $\begin{array}{l}\text { Hugues de Saint-Victor, In } \\
\text { hierarchiam celestem, PL } 175\end{array}$ \\
\hline
\end{tabular}




\begin{tabular}{|c|c|c|}
\hline 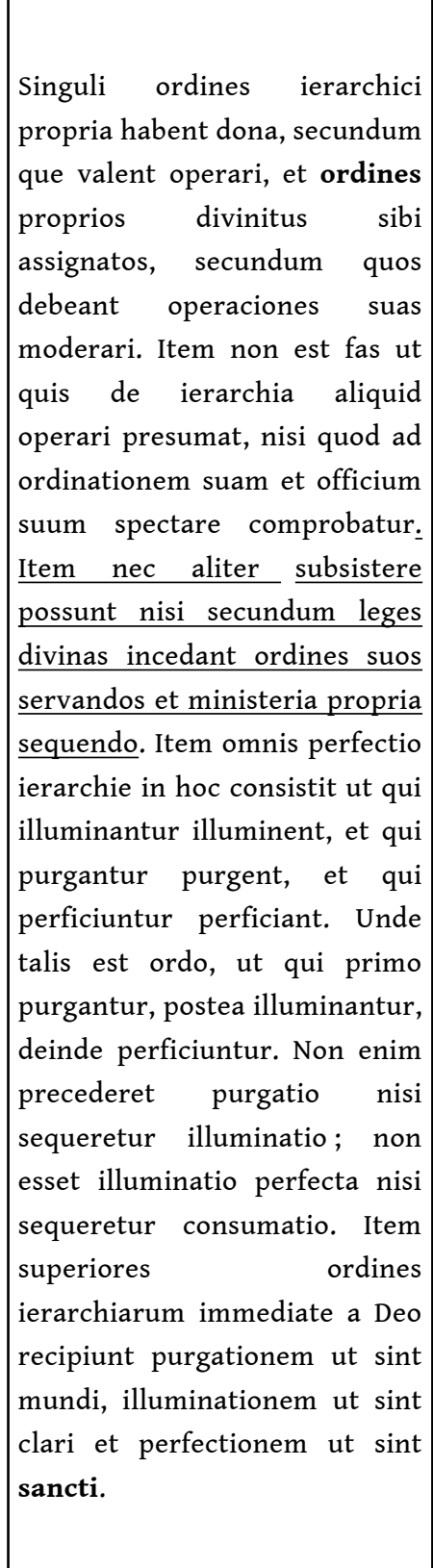 & 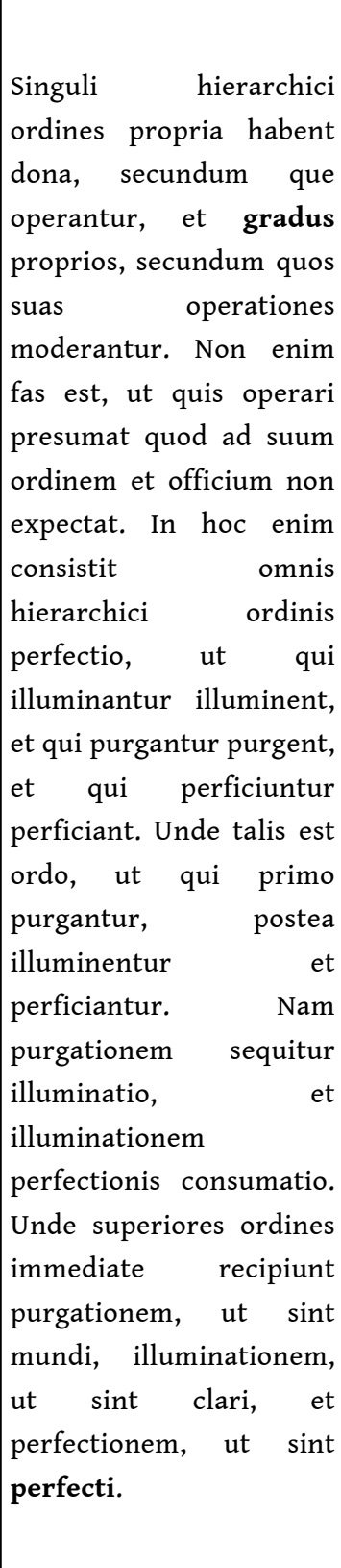 & $\begin{array}{l}\text { (996a) Habent namque singuli } \\
\text { propria dona, secundum que } \\
\text { valeant operari, et ordines } \\
\text { proprios secundum quos debeant } \\
\text { operationem suam moderari. } \\
\text { (996d) Neque fas est etiam sive his } \\
\text { sive illis aliquid operari preter } \\
\text { sacras ordinationes suas, hoc est } \\
\text { preter id quod ad sacras } \\
\text { ordinationes suas spectat, ut } \\
\text { videlicet id solum unusquisque } \\
\text { operari presumat, quod ad } \\
\text { ordinem et officium sibi assignatos } \\
\text { spectare probatur. (997a-b) Aliter } \\
\text { nullatenus subsistere possunt in } \\
\text { eo bono quod appetunt, nisi } \\
\text { secundum leges divinas incedant, } \\
\text { ordinationes suas servando et } \\
\text { ministeria propria exsequendo. } \\
\text { (998b-c) Sic ergo perfectio constat } \\
\text { hierarchie, ut qui purgantur } \\
\text { purgent, et qui illuminantur } \\
\text { illuminent, et qui perficiuntur } \\
\text { perficiant. [...] Primum purgantur, } \\
\text { postea illuminantur, deinde } \\
\text { perficiuntur. Nisi enim precederet } \\
\text { purgatio, non sequeretur } \\
\text { illuminatio; et nisi esset } \\
\text { illuminatio, non veniret } \\
\text { consummatio. [...] Sed sunt } \\
\text { superiores et sublimes et ipsi } \\
\text { appropinquantes divinitati } \\
\text { immediate ab ipsa accipientes et } \\
\text { purgationem ut sint mundi, et } \\
\text { illuminationem ut sint clari, et } \\
\text { perfectionem ut sint sancti. }\end{array}$ \\
\hline
\end{tabular}

On retrouve cet état de choses également dans le livre de l'Experimentator consacré aux corps célestes, avec lequel Barthélemy a en commun de nombreux passages. Pour le texte de l'Experimentator, nous avons collationné deux manuscrits de la rédaction I (Berlin, Deutsche Staatsbibl., Hamilton 277 et Stuttgart, Württembergische Landesbibliothek, med. et phys. $2^{\circ} 24$ ), deux manuscrits de la rédaction II (Chambéry, Bibl. Mun., 30 et Soissons, Bibl. Mun., 26 (28)) et, pour la partie commune, la version abrégée du manuscrit de Wolfenbüttel, éditée par J. Deus. La version I comme la version II comportent le même texte pour le livre réservé aux celestia. Aucun manuscrit de l'Experimentator n'a pourtant conservé la totalité des marqueurs qui sont mentionnés par Barthélemy, ce qui pourrait être dû au fait que, placés en marge dans la version primitive, ils ont été ensuite perdus.

26 La comparaison des deux compilations montre que l'Experimentator a toujours un texte plus correct, plus complet et plus fidèle au texte-source que ne le conserve Barthélemy 
l'Anglais. Pour illustrer cette distance, nous avons sélectionné quelques phrases provenant du commentaire de Rémi d'Auxerre du De nuptiis Philologice et Mercurii de Martianus Capella, des Meteorologica d'Aristote et de l'Hexaëmeron de Robert Grosseteste ${ }^{31}$ :

DPR VIII.17, 1. 162 : Solis constellatione homines pulchros et agiles esse.

Exper. III.26, p. 306 : Dicunt et mathematici Solis constellatione afflatos, pulchros et agiles esse.

R. Aux. 28.13 : ferunt mathematici solis constellatione afflatos, pulchros et celeres

fore.

DPR VIII.27, 1. 9 : Sol in mense Iunio per Pliades iter facit.

Exper. III.19, p. 305 : Sol in mense Maio in Tauro positus per Pliades iter facit.

R. Aux. 22.13 : Sol cum mense Maio in Tauro positus per Pliadas iter facit.

DPR VIII.8, 1. 25-31 : Dicit ergo Aristoteles sic: Ignis propinquus orbi est inflammatus et lucidus et in locis, ubi videtur galaxias, sunt stelle multe parve et luminose, et in illis relucet fulgor ille, et ideo apparet locus ille magis radiosus nec recedit ab uno loco orbis. Hucusque Aristoteles in libro I Metheororum, capitulo II. Exper. Ch f. 53va, St 62r : Dicit ergo Aristoteles quod galaxias sic fit quia ignis purus propinquus orbi est inflammatus et lucidus et in locis in quibus videtur galaxias sunt stelle multe, parve et spisse, propinque et luminose. Cum ergo procedit lumen earum ex illo loco inflammato et ignito videtur in eo lumen oblongum et quia iste stelle sunt fixe et sunt suscipientes splendorem ex Sole continuatur lumen quarundam earum cum quibusdam. Propter illud ergo videtur galaxias in uno loco orbis non recedens ab eo, ut dicit Aristoteles in libro Methaurorum capitulo secundo.

Arist. Meteor. I.4. p. 20-22 : Nos autem dicimus quod esse galaxiae est hoc modo quod est quia ignis purus propinquus orbi est inflammatus lucidus, et in locis in quibus videtur galaxia orbis sunt stellae multae parvae spissae, et magnae propinquae luminosae. Cum ergo procedit lumen earum ex illo loco inflammato ignito, videtur in eo lumen oblongum. Et istae quidem stellae sunt stellae fixae, quarum quaedam tangunt alias, et sunt suscipientes splendorem ex sole. Quare continuatur lumen quarundam earum cum quibusdam. Propter illud ergo videtur galaxia in loco uno orbis non recedens ab eo.

DPR VIII.29, 1. 179-183 : Lux enim multiplicat se gignendo. Gignit namque lux lucem, et lux genita simul gignitur et generat lucem sibi succedentem, et sic in uno instanti unus punctus replet lumine totum orbem.

Exper. Ch f. 58ra, St f. $67 \mathrm{r}$ : Naturaliter enim lux se multiplicat gignendo, et, simul cum est, generat. Quapropter replet circumstantem locum subito. Lux prior secundum locum gignit lucem sequentem; et lux genita simul gignitur et gignit lucem sibi proximo succedentem et illa succedens adhuc ulterius succedentem. Et sic in uno instanti unus lucis punctus replere potest orbem lumine.

R. Gros. Hex. II.10.1, p. 97, 1.28 - p. 98, 1.1: Naturaliter enim lux undique se multiplicat gignendo, et simul cum est generat. Quapropter replet circumstantem locum subito; lux enim prior secundum locum gignit lucem sequentem; et lux genita simul gignitur et est et gignit lucem sibi proximo succedentem; et illa succedens adhuc succedentem ulterius; et ita consequenter. Unde in instanti uno unus lucis punctus replere potest orbem lumine.

DPR VIII.31, 1. 25-29 : Lumen ad superficie polite materie pertingens in angulis equalibus se reflectit et omnium subiectorum formas ibi imprimit, unde quod extra est per substantiam in superficie speculi collocat per imaginem et figuram. VIII, 29, 1. 196-199: Hec, ut dicit Basilius, idem est angelorum et sanctorum supra celum quietissima habitatio. Hec summe Trinitatis per exemplum est demonstratio manifesta.

Exper. Ch f. 58ra-b, St f. 67r: Lux quoque ad corporum politorum superficies pertingens in angulis reflectitur equalibus omniumque corporalium formas obiectas ibi representat. Et quod extra est per substantiam in corpore speculari collocat per ymaginem et figuram. Hec est, ut dicit Basilius, angelorum et sanctorum habitantium supra celum primum diffusa habitatio quietissima. Hec in omnibus 
corporalibus summe Trinitatis ymaginis per exemplum demonstratum manifesta est.

R. Gros. Hex. II.10.4, p. 100, 1. 8-14 : Lux quoque ad corporum politorum superficies in angulis reflectitur equalibus, omniumque corporalium formas et ymagines ubique representat, et quod in uno loco est per substanciam in omni loco dinumerat et collocat per ymaginem et formam. Hec est angelorum et sanctorum, ut testatur Basilius, supra celum primum diffusa habitacio quietissima; hec in rebus corporalibus summe Trinitatis per exemplum demonstracio manifestissima. dans les quatre manuscrits de l'Experimentator que nous avons choisis, montre que le manuscrit de Stuttgart, bien qu'assez fautif, contient une phrase qui est absente des trois autres, mais présente dans le DPR de Barthélemy ${ }^{32}$. En comparant les extraits de l' Hexaëmeron moissonnés par l'Experimentator avec l'édition critique du texte, on constate qu'ils se rapprochent le plus souvent des leçons du manuscrit Oxford, Bodl. Library th. c. 17, qui, sans être le manuscrit autographe, comporte, de l'avis des éditeurs, de nombreuses corrections et annotations de la main de Grosseteste lui-même. L' Experimentator porte la trace de ces corrections à deux endroits au moins. Ainsi, dans le chapitre consacré à la couleur, la relation entre l'action de la lumière et la formation de la couleur est formulée dans ces termes: lux colori superfusa movet eum in generationem sue speciei. Chez Grosseteste on lit: lux colori superfusa movet eum in generacionis sue speciei actum $^{33}$, où superfusa constitue une correction de la main de Grosseteste pour superflua. Sans exclure la possibilité que certains passages du texte de Grosseteste aient circulé, avant ou immédiatement après sa rédaction, sous forme d'extraits ou de reportationes, on peut avancer l'hypothèse que la version utilisée par l'Experimentator est dépendante du manuscrit de l'Hexaëmeron conservé à la Bodleian Library.

es transformations subies par les textes d'Aristote, Rémi d'Auxerre et Robert Grosseteste sous la plume de Barthélemy, il est peu probable que ce dernier ait servi de source à la compilation originale de l'Experimentator, qui, par ailleurs, a emprunté à Rémi d'Auxerre un nombre beaucoup plus grand de citations qu'on ne le constate chez Barthélemy. Il est également peu probable que Barthélemy ait pu utiliser un autre corpus d'extraits que celui de l'Experimentator, puisque les passages communs aux deux compilateurs sont très nombreux et s'étalent sur plusieurs livres. On pourrait donc considérer que c'est par ce biais que Barthélemy cite Martianus Capella, dont le nom cache en réalité celui de Rémi d'Auxerre. Plus d'une trentaine de citations de Rémi d'Auxerre sont ainsi tirées du commentaire des deux premiers livres du De nuptiis, qui ont souvent circulé séparément ${ }^{34}$.

D'autres citations rubriquées Martianus ont été moissonnées par Barthélemy à partir de la compilation d'un autre contemporain, Arnold de Saxe, qui lui a fourni trois citations du livre VIII du De nuptiis, traditionnellement connu comme Liber de astrologia/ astronomia. Nous verrons plus bas à propos d'Arnold de Saxe que la compilation attribuée à l' Experimentator partage également avec lui et Barthélemy des citations du De motibus/ iudiciis planetarum attribue à Ptolémée.

Dans le cas d'Isidore, la formulation de l'Experimentator, qui se retrouve presque à la lettre chez Barthélemy, s'éloigne du texte original, ce qui laisse supposer, comme pour Martianus Capella, l'utilisation d'une version glosée des Étymologies. C'est ce qui pourrait expliquer que le chapitre sur les cercles de la sphère mis sous l'autorité d'Isidore a une grande ressemblance avec la Philosophia de Guillaume de Conches. Par ailleurs, les passages étymologiques concernant les signes du Zodiaque ${ }^{35}$ n'ont pas de correspondant 
dans l'œuvre d'Isidore, même si quelques liens peuvent être tracés avec le contenu du livre III, c. 71 des Étymologies. Ce type d'étymologie fondée sur l'imaginaire mythographique est attesté depuis l'Antiquité et se retrouve dans de nombreux textes tout au long du Moyen Âge, que ce soit le Computus d'Helperic d'Auxerre, la Sphera de Jean de Sacrobosco, le Liber introductorius de Michel Scot ou le petit compendium du manuscrit Città del Vaticano, Vat. lat. $4082\left(\mathrm{~s} . \mathrm{XV}^{\mathrm{in}}\right)$ édité par L. Thorndike ${ }^{36}$. Néanmoins, aucun de ces textes ne se rapproche des définitions recueillies par l'Experimentator et par Barthélemy l'Anglais. C'est dans un corpus de gloses sur le De natura rerum de Bède le Vénérable que nous avons retrouvé quelques formulations étymologiques identiques à celles présentes dans les compilations du XIII ${ }^{\mathrm{e}}$ siècle. Rédigées au début du $\mathrm{X}^{\mathrm{e}}$ siècle, ces gloses ont été intégrées plus tard dans un autre corpus que l'on a attribué à Byrhtferth de Ramsey. Pour les signes du Zodiaque, seule la description du Verseau correspond au texte cité par l'Experimentator et par Barthélemy. En voici la teneur :

DPR VIII.10, 1. 226-228: Aquarius secundum fabulas pincerna deorum manibus eorum aquam infundens, unde tenet urnam.

Exper., III.8, p. 303 : Aquarius secundum fabulas dicitur pincerna deorum manibus eorum aquam infundens. Unde urnam tenet.

Gloses DNR: Aquarius dicitur Ganimedes qui est pincerna deorum et in eius manibus aquam fundit. Unde et urnam tenet.

C'est parmi ces gloses que nous avons également identifié une définition de l'étoile polaire que les encyclopédistes attribuent à Bède.

DPR VIII.23, 1. 2-3 : Polus, ut dicit Beda, est stella parvissima, a qua tota pars celi superior est sic dicta.

Exper., III.15, p. 304 : Polus est stella parvissima, a qua tota illa superior pars celi dicta est.

Gloses DNR: Polus dicitur stella parvissima in vertice septentrionis, a qua tota illa pars celi polus vocatur.

Tout comme le texte de Martianus Capella est cité à travers son commentateur, les passages associés au marqueur Beda proviennent probablement d'un fonds de gloses placées en marge du De natura rerum. Que ce soit pour Martianus Capella cité via Rémi d'Auxerre, pour Denys l'Aréopagite cité via Hugues de Saint-Victor, pour Bède cité via des gloses anonymes, l'Experimentator fait un usage plus large de ces corpus de commentaires que Barthélemy. Si les comparaisons que nous avons effectuées et discutées ci-dessus - et qui pourraient être multipliées - étayent l'hypothèse d'une utilisation de la compilation dudit Experimentator par Barthélemy l'Anglais, la relation entre les deux textes doit être davantage étudiée. À partir des sondages que nous avons menés sur l'ensemble des deux encyclopédies, nous avons constaté que le nombre de citations communes n'est plus du tout aussi important dans les sections consacrées aux «règnes " minéral, végétal et animal. Cela supposerait, soit que Barthélemy ait eu accès à une version encore en chantier du texte de l'Experimentator, soit que, pour les livres sur les « règnes naturels », il lui ait préféré d'autres textes, soit encore qu'en quittant la bibliothèque où il a compulsé le recueil de l'Experimentator, il n'ait pas pu emmener une copie avec lui.

Par ailleurs, au sujet du lieu de rédaction de ce recueil, J. Deus n'a avancé aucune hypothèse. Des références à Guillaume d'Auxerre, la présence de citations extraites de l' Hexaëmeron de Robert Grosseteste et de plusieurs traités d'Aristote, qui sont toujours plus fidèles à l'original que celles fournies par Barthélemy l'Anglais, appuyeraient l'hypothèse que l'Experimentator aurait élaboré sa compilation au tout début des années 1230. Mais, pour l'instant, les données sont insuffisantes pour nous permettre de localiser sa 
rédaction. Tout au plus peut-on dire qu'elle vit le jour quelque part en France du Nord ou en Angleterre.

\section{Un intermédiaire saxon : Arnoldus Luca/Saxo}

Un autre auteur qui a servi à Barthélemy d'intermédiaire est Arnold de Saxe ${ }^{37}$. Son nom n'est jamais mentionné dans le De proprietatibus rerum ni dans la table des auteurs. Pourtant, son De floribus rerum naturalium, écrit vraisemblablement autour de 1225-1235, a été la source directe de Barthélemy pour nombre d'auteurs cités sous forme de marqueurs de référence au livre VIII ${ }^{38}$. Toutes ces citations empruntées à la lettre, mais souvent simplifiées quant aux termes techniques, sont tirées des livres I, II, III du De celo et mundo, qui constitue la première des cinq parties du De floribus rerum naturalium d'Arnold de Saxe, une partie malheureusement conservée seulement dans l'unique manuscrit complet du De floribus, Erfurt, Wissenschaftl. Allgemeinbibl., Ampl. oct. 77 et partiellement dans les miscellanées astronomiques du manuscrit Basel, Univ. bibl., O.IV.4. C'est le cas par exemple des trois citations du Liber celi et mundi, paraphrase d'un livre de la Physique du Shifā d'Avicenne, attribuée à Aristote. À travers ces passages copiés sur Arnold de Saxe, Barthélemy l'Anglais cite également le De sensu d'Aristote ${ }^{39}$. Sept passages sont également empruntés à travers Arnold de Saxe au De celo et mundo "secundum novam translationem » d'Aristote, détail qui fait référence à la traduction de Gérard de Crémone. C'est aussi le cas des cinq citations du De proprietatibus elementorum pseudo-aristotélicien, dont le découpage a le même périmètre que dans le De floribus. Deux autres citations éloignées $d u$ texte $d u$ Pseudo-Aristote semblent être tirées elles aussi du même intermédiaire, sans doute un état du texte d'Arnold de Saxe qui n'a pas été conservé.

Des textes d'astronomie plus classiques proviennent également du De floribus rerum naturalium : trois citations du livre VIII du De nuptiis de Martianus Capella, quatre du Liber Ciceronis (Commentarii in Somnium Scipionis) de Macrobe, et une citation du Liber Thymei de Platon dans la traduction de Chalcidius.

De la compilation d'Arnold sont aussi empruntés, simplifiés et abrégés les quinze passages mis sous le nom d'Albumasar, De motibus astrorum / planetarum (un seul présent chez Arnold de Saxe n'a pas été repris par Barthélemy) et les quatorze citations d'un De motibus et iudiciis planetarum, attribué à Ptolémée, qui apparaît chez Barthélemy sous le titre De iudiciis astrorum. Nous avons déjà retracé ailleurs la diffusion et offert une édition de cette dernière source, dont Arnold de Saxe est le meilleur témoin connu ${ }^{40}$. Au fil des recherches menées pour l'édition du livre de Barthélemy, nous nous sommes aperçus que des citations du Pseudo-Ptolémée sont également présentes dans la compilation dudit Experimentator, où les informations ne sont pas aussi complètes que chez Arnold de Saxe. Quelques mots supplémentaires et un détail terminologique singularisent pourtant la tradition de l'Experimentator. Il s'agit du terme Chelae utilisé pour désigner le signe de chute de la Lune, Chelae qui dans la tradition astronomique aratéenne représente l'astérisme des pinces du Scorpion. Ce terme est remplacé dans la version présente chez Arnold de Saxe par celui de Libra ${ }^{41}$, ce qui laisse croire que l'Experimentator, en employant un archaïsme, n'est pas dépendant de la compilation d'Arnold de Saxe. De plus, le chapitre sur la Lune tiré du Pseudo-Ptolémée comporte chez l'Experimentator un ajout relatif aux phases de la Lune qui se retrouve à la lettre dans les Proportiones competentes in astrorum industria $a^{42}$. Malgré ces différences, il est certain que plusieurs textes sont communs à l'Experimentator, Arnold de Saxe et, par leur entremise, à Barthélemy l'Anglais. Si notre hypothèse d'une utilisation de la compilation de l'Experimentator par 
Barthélemy se confirme, il faut imaginer que Barthélemy a dû retrouver dans le texte de l' Experimentator des citations du Pseudo-Ptolémée qu'il avait aussi lues chez Arnold de Saxe.

Nos remarques sur l'utilisation de la compilation de l'Experimentator s'appliquent aussi aux textes empruntés à Arnold de Saxe, que Barthélemy ne conserve pas toujours à l'identique. Lorsqu'il reprend le De motibus astrorum associé au nom d'Albumasar, texte qui s'apparente à la littérature des parapegmata, il ne sélectionne pas toute l'information concernant l'influence de telle ou telle constellation, et découpe de façon inappropriée les phrases, ce qui a pour effet de transposer les propriétés d'une constellation sur telle autre. Il en va de même pour les citations provenant d'Aristote. Barthélemy suit en grande partie l'assemblage constitué par Arnold de Saxe, mais prend parfois l'initiative de modifications. Par exemple, au sujet de la vie dans le ciel, Barthélemy change la place d'une glose enchâssée par Arnold dans le texte d'Aristote, perd en cours de route un mot et remplace un adjectif pour donner un sens plus théologique à l'idée exprimée par le texte :

DPR, VIII.2, 1.104-106: Unde illic est vita fixa, scilicet extra ultimum, et est sempiterna que neque deficit neque finitur, et illa est vita vera.

Ar. Saxo, I.11, cit. 2: Vita ergo illic, id est extra ultimum incessum, est fixa, sempiterna. Non finitur nec deficit, et est melior vita.

Arist. De celo 279a : Vita ergo illic est fixa sempiterna in saecula saeculorum, quae non finitur neque deficit, et est melior vita.

Ailleurs, Barthélemy met sous un seul marqueur, Liber de celo et mundo, des citations qu'Arnold de Saxe départage et rubrique avec les marqueurs respectifs liber de celo et mundo secundum novam translationem, qui correspond à la traduction du De celo faite par Gérard de Crémone, et Liber de celo et mundo secundum veterem translationem, qui cache en fait le traité Liber celi et mundi de Pseudo-Avicenne ${ }^{43}$. En outre, en utilisant des compilations déjà disponibles, Barthélemy introduit parfois un même passage à deux reprises, comme il arrive pour une phrase sur la musique céleste, tirée du Songe de Scipion de Macrobe, qui conserve à un endroit le même découpage que chez Arnold de Saxe, alors qu'à un autre, elle est formulée un peu différemment ${ }^{44}$.

39 Le De floribus rerum naturalium, ou bien le florilège qu'Arnold de Saxe dit avoir rédigé antérieurement, mais qui n'a pas été retrouvée ${ }^{45}$, a par ailleurs été exploité par Barthélemy à plusieurs autres reprises en-dehors des questions astronomiques, que ce soit dans le domaine des pierres ou celui des animaux ${ }^{46}$.

Cela implique une hypothèse de localisation de la rédaction du livre VIII du De proprietatibus rerum: le séjour de Barthélemy à Magdebourg au début des années 1230 comme frère franciscain enseignant au nouveau studium ${ }^{47}$ de Theutonia aurait été l'occasion de puiser à la compilation d'Arnold de Saxe, état qui se complète grâce aux sources découvertes à Paris, qu'il s'agisse de l'Experimentator, d'Alexandre de Halès ou des questiones concernant la lumière dont il sera question ci-dessous. Quelques années plus tard, Vincent de Beauvais et Albert le Grand, qui ont tous deux utilisé la compilation d'Arnold de Saxe, n'ont quant à eux pas eu à se déplacer à Magdebourg pour connaître une partie du De floribus rerum naturalium ${ }^{48}$. On peut supposer même que Barthélemy soit le vecteur du transfert du De floribus entre Magdebourg, où a probablement enseigné Arnold de Saxe ${ }^{49}$, et Paris. Compte tenu du contexte socio-politique parisien, Barthélemy serait parti à Magdebourg lors de la grève universitaire (1229-1231) et serait rentré à Paris lorsque la situation s'est apaisée suite aux effets de la bulle Parens scientiarum de 
Grégoire IX (1231), mais il a semble-t-il repris ensuite sa carrière en Saxe, où il aurait terminé son encyclopédie ${ }^{50}$.

\section{L'influence des théologiens anglais}

Robert Grosseteste (1175-1253) et Alexandre de Halès (1180-1245)

41 Comme il a été déjà souligné par les éditeurs d'autres livres du DPR, quelques sources inviteraient à considérer attentivement l'influence anglaise sur l'encyclopédie de Barthélemy. Dans le livre VIII, nous avons repéré quelques indices supplémentaires qui appuient l'hypothèse d'emprunts faits aux écrits de maîtres anglais. Ainsi, sans le citer nommément, et par le biais de la compilation de l'Experimentator, Barthélemy a utilisé l' Hexaëmeron de Grosseteste ${ }^{51}$. Ce commentaire fut rédigé, selon les conclusions des éditeurs, à la fin de la carrière professionnelle de Grosseteste, entre 1232 et $1235^{52}$, ce qui imposerait ce terminus post quem à l'Experimentator. Comme le concluent d'autres études ${ }^{53}$, Barthélemy a aussi repris à la lettre presque la totalité du De colore de Grosseteste lorsqu'il a traité de ce sujet au premier chapitre du livre XIX. Pour mémoire, l'Hexaëmeron serait de peu postérieur au De luce et au De colore. Le De luce aurait été écrit vers 1225-1228 (c. 1235-1240 d'après R. Southern), et les traités De iride, De colore, De calore solis, De natura locorum auraient été rédigés c.1230-1235, d'après les avis confirmés de J. McEvoy et R. C. Dales ${ }^{54}$.

Les silences de Barthélemy à propos de diverses notions scientifiques abordées par Grosseteste tendent à conclure que notre auteur n'a pas eu accès, pour la rédaction du livre VIII, à d'autres œuvres de son contemporain. Cependant, il est possible de tracer quelques rapprochements avec certaines d'entre elles (De luce, De natura locorum par exemple) ou avec des discussions issues de l'enseignement de Grosseteste, sans qu'on puisse améliorer la chronologie relative de Grosseteste grâce à celle de Barthélemy, ou vice versa. Nous revenons ci-dessous sur ces similitudes dans la partie consacrée au contenu du De mundo à propos du diaphane, de la couleur et de la lumière.

En effet, la diffusion des traités de Grosseteste reste un sujet débattu. On sait qu'il s'était lié avec la plupart des Anglais qui étudiaient à Paris, ainsi qu'avec Guillaume d'Auvergne. On garde notamment plusieurs lettres où l'évêque de Paris s'adresse à lui comme amicus carissimus et leurs vues philosophiques se ressemblent sur divers points, entre autres sur la théorie de la lumière ${ }^{55}$. Le chroniqueur franciscain Salimbene de Adam, en dressant la liste des amis d'Hugues de Digne (ministre provincial des Franciscains en Provence), y mentionne Grosseteste aux côtés d'Adam Marsh et d'Alexandre de Halès, qui fut maître régent à Paris entre 1220 et 1245 , année de sa mort ${ }^{56}$. Tous ces contacts remontent probablement à la décennie 1220-1230, même si aucun document de la pratique n'a été retrouvé pour attester le passage de Grosseteste à Paris pendant cette période ${ }^{57}$. On a généralement considéré qu'il avait enseigné à Oxford dans les années 1220, mais il n'y a aucune preuve qu'il y ait enseigné la théologie avant $1230^{58}$. Il se trouvait en tous cas à Oxford lors de la visite de Jourdain de Saxe en 1229, et il y enseignait en 1231 et en 1234. Il existe également des indices montrant qu'il a enseigné la théologie aux Franciscains d'Oxford dès 1229 et jusqu'en $1235^{59}$. D'autre part, si l'on admet l'attribution à Grosseteste du De anima du manuscrit Oxford, Bodl. Libr., Digby 104, écrit semble-t-il après 1230, on admet du même coup des liens doctrinaux avec Philippe le Chancelier et l'influence de la Summa de bono de ce dernier, écrite entre 1225 et $1228^{60}$. 

l'autre de ses traités ou des traductions qu'il a réalisées. La présence de citations provenant de ses œuvres dans les compilations de l'Experimentator et de Barthélemy l'Anglais ainsi que dans la Summa 'Alexandri de Hales' constitue en effet un indice de leur réception immédiate. Vu les liens entretenus par Robert Grosseteste avec les écoles parisiennes, on peut faire l'économie de l'hypothèse que l'Experimentator et Barthélemy aient connu son œuvre en Angleterre.

Quant à la Somme théologique dite d'Alexandre de Halès, elle a été écrite collectivement à Paris à partir de 1235, notamment avec la collaboration de Jean de la Rochelle et d'Eudes Rigaud $^{61}$. Vingt ans plus tard, l'ouvrage était toujours en chantier, lorsqu'une bulle d'Alexandre IV presse le ministre de la province franciscaine de France (Francia) d'envoyer auprès de Guillaume de Méliton des frères afin de l'aider à achever la somme d'Alexandre, mort en $1245^{62}$. Puisque le De proprietatibus rerum cite à la lettre certains passages de la Summa, il est probable que Barthélemy l'ait connue lors d'un séjour à Paris, dans le milieu franciscain.

\section{Richard Fishacre ( $\$ 1248)$, Richard Rufus (fl. 1238-1259)}

La question de l'influence du milieu anglais devient encore plus complexe lorsqu'on constate les emprunts faits par Barthélemy à la question De luce de Richard Fishacre, emprunts déjà brièvement signalés par les éditeurs de ce texte ${ }^{63}$. Rédigée probablement après 1245 , cette question a une place assez problématique dans la tradition des écrits de Fishacre. Conservée dans deux manuscrits seulement ${ }^{64}$, elle aurait été écrite après la rédaction du commentaire des Sentences, commentaire qui par ailleurs ne semble pas avoir connu une diffusion importante sur le continent. Pourtant, la questio de luce est compilée par Vincent de Beauvais dans son Speculum maius. Elle figure déjà dans la version bifaria du Speculum naturale, terminée vers 1244/124765. Sans être rapporté à un nom d'auteur, le texte est placé sous le marqueur Memoriale rerum difficilium, qui réunit en réalité trois chapitres provenant du De intelligentiis attribué à Adam de Bellefemme et deux autres qui représentent des passages tirés du commentaire des Sentences de Fishacre, y compris de la questio de luce ${ }^{66}$. La présence de ces extraits dans les encyclopédies de Barthélemy l'Anglais et de Vincent de Beauvais permet de supposer que la question traitée par Fishacre a été connue à une date assez précoce et qu'elle était déjà intégrée à son commentaire des Sentences, qui porte la marque de l'influence de Robert Grosseteste ${ }^{67}$.

Cependant, les arguments communs avec la questio de luce apparaissent chez Barthélemy dans une démonstration plus longue. Cela invite à penser que Barthélemy a repris un texte qui, tout en intégrant les éléments abordés par Fishacre, a développé davantage la question de la nature de la lumière, comme l'a fait par exemple Richard Rufus dans son commentaire des Sentences ${ }^{68}$.

En tous les cas, ces emplois contemporains prouvent des passages rapides de l'information philosophique entre les cercles anglais et parisiens, entre franciscains et dominicains, qui pourraient sans doute être mieux documentés par une étude des autres questions traitant de la lumière. En guise de première piste, nous avons constaté que certains manuscrits témoignent, par leur Mitüberlieferung, des connivences entre ces auteurs $^{69}$. Ainsi, le manuscrit Assisi, Bibl. comunale 138 contient une collection de questions disputées (surtout théologiques) qui reflètent l'enseignement franciscain à 
Paris dans le second quart du XIII ${ }^{\mathrm{e}}$ siècle. Il a été composé autour de 1250 et porte dans les marges les traces de notes faites par Bonaventure ${ }^{70}$. Aux f. 249r-261v, il contient des reportationes de questions philosophiques qui proviennent probablement d'un seul auteur -qui n'est pas Richard Rufus- et qui ont été rédigées entre 1230 environ et 1257 par un maître ès arts parisien ou oxonien. Ces questions anonymes sont suivies d'autres questions attribuées à Robert Grosseteste, Richard Rufus et peut-être Richard Fishacre, copiées de la même main. Par ailleurs, une série de questions traitant de lucis natura, de perspicuo, de radio, de calore solis composées dans la première moitié du XIII ${ }^{\mathrm{e}}$ siècle et influencées par les théories de Robert Grosseteste se trouve insérées dans le commentaire de la Metaphysique de Richard Rufus conservé dans le manuscrit Salamanca, Bibl. universitaria $2322^{71}$.

Les ressemblances, que nous signalons plus loin, entre les arguments évoqués au sujet de la lumière par Barthélemy l'Anglais, Richard Fishacre et Bonaventure invitent à mener des recherches plus poussées dans la littérature scolastique franciscaine des années 1230-1250 afin de retrouver les textes que Barthélemy a pu explorer.

\section{L'apport des nouveaux outils d'exégèse et d'indexation : la glose ordinaire et la concordance biblique}

Invention majeure du XIII ${ }^{e}$ siècle, la concordance biblique facilita le repérage rapide des occurrences d'un mot dans les livres de l'Ecriture sainte. Structuré selon l'ordre alphabétique, cet index associait chaque mot à un verset biblique identifié par le sigle du livre biblique, par le chapitre et par une lettre qui positionnait le verset à l'intérieur du chapitre. Ce dernier élément d'indexation se fondait sur une division du chapitre en sept parties, numérotées de a à g. De la sorte, tout mot répertorié dans les concordances pouvait être retrouvé à l'intérieur d'un chapitre avec une certaine précision. Après la division en chapitres du texte biblique proposée au tout début du XIII siècle probablement par Étienne Langton - et adoptée sous une forme modifiée à Paris

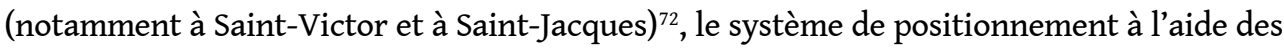
sept lettres facilitait davantage encore l'accès au texte. Il sera consacré par les diverses concordances qui ont vu le jour au XIII ${ }^{\mathrm{e}}$ siècle au couvent Saint-Jacques ${ }^{73}$.

51 Quelques passages de la compilation de Barthélemy l'Anglais laissent croire qu'il a utilisé la postille d'Hugues de Saint-Cher et une des concordances bibliques rédigées à l'époque ${ }^{74}$. Hormis quelques très rares exceptions, la division par lettres n'apparaît pas dans les manuscrits de la Bible qui subsistent aujourd'hui, ce qui pousserait à croire que les occurrences de ce système que nous avons repérées chez Barthélemy attestent de l'usage d'une concordance ${ }^{75}$ : était-ce celle attribuée à Hugues de Saint-Cher ou celle de Richard de Stavensby?

52 Les quelques rapprochements avec la postille hugonienne nous portent à croire que Barthélemy a eu accès aux outils élaborés au couvent dominicain de Saint-Jacques. Ainsi, au livre VIII du DPR, une citation du Deutéronome est le lieu d'une digression sur la typologie des cieux qui est, de l'aveu de Barthélemy, empruntée à la glose ${ }^{76}$. L'enchaînement apparaissant à la fin de cette glose met en relation le verset du Deutéronome et un verset de Matthieu (13.4 ${ }^{77}$, rapprochement que nous n'avons repéré que dans la postille d'Hugues de Saint-Cher ${ }^{78}$. Le texte censé reprendre la glose ne correspond pourtant ni à la glose ordinaire ni à la postille. Le commentaire qui chez Barthélemy explicite la typologie des cieux, ne place plus les oiseaux et les démons dans 
le ciel aéré comme le faisait traditionnellement l'exégèse, mais bâtit sa description sur la physique des vapeurs.

La rédaction de la concordance que l'on a souvent associée au nom d'Hugues de SaintCher était probablement en cours autour de 1235, l'utilisation de cet outil d'indexation étant attestée dans la postille aux Epîtres de Paul ${ }^{79}$. Récemment, Paul Saenger a cherché à montrer que cet outil se situe dans la lignée d'autres procédés d'ordonnancement développés dans les milieux anglais. Saenger met Thomas Gallus à l'origine du système des lettres de l'alphabet et tente de montrer que ce sont les Dominicains anglais qui se trouvaient parmi les inventeurs des premières concordances bibliques ${ }^{80}$. Les récents travaux que Declan Lawell a menés sur les écrits de Thomas Gallus vont dans le même sens. Le plus ancien manuscrit des commentaires de l'ensemble du corpus dyonisien (Città del Vaticano, B.A.V., Arch. Cap. S. Pietro D 181, daté de 1270), qu'il a achevés en 1244, conserve toutes les références au système alphabétique, tout comme les Glose super Angelica Ierarchia rédigées en $1224^{81}$. Bien plus, Thomas Gallus applique ce même procédé aux traités de Pseudo-Denys l'Aréopagite ${ }^{82}$. Un autre théologien des années 1230, Roland de Crémone, utilise lui aussi constamment dans sa Summa ce système d'indexation ${ }^{83}$. Ces divers témoignages prouvent que le système était en usage au moment où Barthélemy écrivait le $D P R$.

L'hypothèse que les copistes de ces traités auraient introduit ultérieurement les lettres de l'alphabet n'est pas à écarter. Dans ce cas, en ce qui concerne le DPR, on ne s'explique pas pourquoi le système n'a pas été appliqué d'un bout à l'autre de la compilation. En revanche, si les lettres ont été insérées dans le texte dès le départ, on comprend mal pourquoi Barthélemy ne les a lui-même adoptées qu'à quelques endroits. Il est certain par ailleurs que plusieurs citations bibliques ont été importées par Barthélemy par source interposée, ce qui expliquerait dans ces cas l'absence du système alphabétique. Barthélemy aurait moissonné lui-même un certain nombre d'autres versets afin d'illustrer son propos; dans ces cas, il a, selon toute apparence, fait usage d'une concordance et intégré les références dotées des lettres de l'alphabet. La présence dans le DPR d'autres textes d'Hugues de Saint-Cher rend plausible l'hypothèse que Barthélemy a été parmi les premiers à avoir mis à profit les nouveaux outils d'indexation élaborés à Paris.

\section{Auteurs astrologiques énigmatiques : Ptolémée et Misael, de Raymond de Marseille à Michel Scot}

\section{Michel Scot (c. 1175 - post 1232)}

55 A la recherche des sources de Barthélemy l'Anglais, nous avons rencontré des marqueurs de référence au sujet desquels la bibliographie scientifique n'a pas été d'un grand secours. Il faut dire que, pour certains d'entre eux, le DPR est le seul témoin, aucun manuscrit ne leur ayant été associé par les catalographes médiévaux ou contemporains. Ainsi, dans le chapitre 10 consacré aux planètes, plusieurs citations sont mises sous le nom de Misael. Lynn Thorndike, dans le résumé qu'il a donné de l'encyclopédie de Barthélemy, faisait l'hypothèse que ce nom soit une déformation graphique de celui de Messahalla, et non de celui de Michel $\mathrm{Scot}^{84}$. Toutefois, l'index des autorités qui figure à la fin des manuscrits du DPR porte la mention du nom de Michael Scotus, qui n'apparaît nulle part tel quel parmi les marqueurs de citation, alors que la forme Misael, citée à répétition au cours de l'exposé, ne figure pas dans cette liste. Qui se cache alors derrière ce Misael ? 
Les éditeurs des deux premiers livres du DPR ont signalé un certain nombre de parallélismes avec le prologue du Liber introductorius de Michel Scot, qui pourraient indiquer que Barthélemy l'Anglais a eu accès à cette introduction à l'astrologie philosophique. Voici, pour compléter le répérage antérieur, la liste des passages qui se recoupent souvent mot pour mot chez les deux auteurs, comparaison faite à partir de l'édition critique récente des livres I et II du DPR et du prologue de Michel Scot édité par G. Edwards ${ }^{85}$ :

\begin{tabular}{|c|c|}
\hline Barthélemy l'Anglais & Michel Scot \\
\hline I. $1,69,1.2-16$ & $105,1.5-20$ \\
\hline I. $2,69,1.3-5$ & $106,1.2-4$ \\
\hline I. $2,70,1.14-25$ & $107,1.3-108,1.3$ \\
\hline I. $4,71,1.2-15$ & $87,1.21-88,1.15$ \\
\hline I. $16,77,1.61-69$ & $182,1.7-13$ \\
\hline II. $1,105,1.2-7$ & $38,1.15-19$ et $21-24$ \\
\hline II. $2,105,1.2-5$ & $113,1.18-21$ \\
\hline II. $2,105,1.2-5$ & $113,1.18-21$ \\
\hline II. $2,106,1.44-50,53-60$ & $129,1.8-11,17-23 ; 129,1.23-1301.7 ; 131,1.7-9$ \\
\hline II.3, 109, 1. 8-25 & $157,1.18-158,1.9$ \\
\hline II.7, $112,1.2-113,1.31$ & $131,1.1-132,1.19 ; 131,1.22 ; 132,1.19-133,1.2$ \\
\hline II.7, $114,1.80-84$ & $133,1.14-19$ \\
\hline II. $8,114,1.4-115,1.14$ & $135,1.12-21,136,1.13-15$ \\
\hline II.9, $117,1.2-6$ & $137,1.3-4,14-17$ \\
\hline II.10, $118,1.2-5$ & $138,1.1-4$ \\
\hline II.12, $120,1.2-5,1.15-17$ & $139,1.1-4,1.6-7$ \\
\hline II.13, $121,1.2-13$ & $140,1.6-14$ \\
\hline II.15, $122,1.2-9$ & $141,1.23-142,1.9$ \\
\hline II. $16,124,1.40-43$ & $143,1.9-11$ \\
\hline II.17, 124, 1. $2-125,1.19$ & $143,1.19-144,1.2 ; 144,1.18-145,1.15$ \\
\hline II.17, $125,1.27-31$ & $145,1.22-146,1.1$ \\
\hline
\end{tabular}




\begin{tabular}{|l|l|}
\hline II.18, 126, 1. 13-27 & $146,1.20-147,1.6 ; 147,1.9-12$ \\
\hline II.19, 129, 1. $66-130,1.87$ & $162,1.11-21 ; 163,1.2-9$ \\
\hline II.19, 130, 1. $95-131,1.106$ & $164,1.20-24$ \\
\hline II.20, 131, 1. $-132,1.18$ & $165,1.5-19$ \\
\hline II.20,132, 1. $41-133,1.43$ & $165,1.21-23$ \\
\hline II.20,133, 1. $53-55$ & $165,1.23-166,1.1$ \\
\hline
\end{tabular}

Souvent, il s'agit de citations tirées des Étymologies d'Isidore, ce qui, vu la place de cette œuvre dans le paysage lexicographique et encyclopédique médiéval, peut s'expliquer par le fait que Barthélemy comme Michel Scot ont utilisé directement cette source. Par contre, les citations qui sont marquées chez Barthélemy comme provenant de Dionysius ne se retrouvent telles quelles dans aucune œuvre du Pseudo-Aréopagite, alors qu'elles sont présentes presque à la lettre chez Michel Scot et, ce qui complique davantage les choses, chez l'Experimentator. Une citation commune avec la Summa 'Alexandri de Hales' nous interpelle elle aussi. Sa présence chez Michel Scot et chez Barthélemy devrait avoir pour conséquence de reculer la datation de l'ouvrage de Michel Scot dans les années 1240.

D'autre part, autant Michel Scot que Barthélemy dans le livre I du DPR font un assez large extrait du Liber extra dans la section consacrée à la Trinitée ${ }^{86}$, ce qui nous conduirait à situer le terminus a quo de leurs œuvres en 1234, date de l'élaboration du recueil des Extravagantes. Cette nouvelle compilation canonique mise au point par le dominicain Raymond de Peñafort à la demande du pape Grégoire IX fut destinée premièrement aux universités de Paris ${ }^{87}$ et de Bologne ${ }^{88}$. Alors que Michel Scot a extrait des Décrétales des passages plus larges des lettres d'Innocent III que ne l'a fait Barthélemy, ce dernier a cependant un texte qui se rapproche, davantage que celui de Michel Scot, de celui des Décrétales.

Les nombreux lieux parallèles entre les œuvres de l'Experimentator, de Barthélemy l'Anglais et de Michel Scot ouvrent la voie à de multiples hypothèses concernant la connaissance qu'ils ont eue les uns des autres. Si le prologue du Liber introductorius est bien de la plume de Michel Scot, trois explications sont envisageables : soit Barthélemy et l'Experimentator l'ont utilisé à plusieurs endroits, soit l'inverse, soit les trois ont exploité des sources communes. Quant à la première hypothèse, il convient de noter que Barthélemy fournit plus fréquemment que Michel Scot l'origine de ses citations, allant jusqu'à indiquer le chapitre concerné. On pourrait supposer, comme nous l'avons fait pour l'Experimentator, que le livre de Michel Scot comportait à l'origine dans les marges toutes ces indications que Barthélemy aurait reprises dans le corps du texte. Si, dans l'hypothèse inverse, on admet que Michel Scot a puisé chez l'Experimentator ou chez Barthélemy, on s'explique mal pourquoi son nom figure dans la table d'autorités citées à la fin du DPR. On pourrait donc supposer que Michel Scot a envoyé son traité dans les milieux parisiens ou qu'il est venu lui-même à Paris, et cela à une date postérieure à 1234, vu l'utilisation qu'il fait du Liber extra.

Pour ajouter un fil de plus à cet entrelacs d'hypothèses, rappelons que Barthélemy l'Anglais a constitué le dossier trinitaire du livre I du DPR non seulement à l'aide des 
citations du Liber extra, mais également en intégrant un long développement emprunté au prologue mis par Hugues de Saint-Cher en tête de son commentaire des Sentences ${ }^{89}$. Cela rend l'exposé de la Trinité par Barthélemy plus «moderne » que celui de Michel Scot et de l'Experimentator, alors qu'en matière d'angélologie, Barthélemy reprend la doxa dyonisienne telle qu'elle est présente chez l'Experimentator et partagée aussi par Michel Scot.

61 Quoiqu'il en soit des éventuels échanges entre ces trois auteurs sur les sujets abordés dans les livres I et II du DPR, nous n'avons réussi à repérer aucune citation du Liber introductorius de Michel Scot, que ce soit dans sa version brève ou longue, dans le livre VIII du DPR, ce qui n'appuie guère l'hypothèse de l'identité entre Misael et Michel Scot.

\section{Misael : Zael et ses intermédiaires}

Sur les traces du mystérieux Misael, nous avons exploré d'autres pistes. En arabe, l'expression "min Sahl» signifie «d'après Zael», ce qui pourrait expliquer la translittération latine Misael ${ }^{90}$. L'homonymie avec l'un des trois compagnons du prophète Daniel y fut peut-être pour quelque chose. Zael (Abū 'Utmān Sahl ibn Bisr ibn Habīb ibn Hāni' al-Isrāâîī) est un des astrologues les plus renommés du IX ${ }^{e}$ siècle ${ }^{91}$. Son nom est décliné, selon les sources, en Zael, Zahel, Tethel ${ }^{92}$, Sehel.

C'est certainement à une de ses œuvres que Barthélemy emprunte, directement ou via un intermédiaire, la définition des notions astrologiques de triplicitas, exaltatio, domus, facies, angulus ${ }^{93}$. Il devrait logiquement s'agir de son Liber iudiciorum (ou Iudicia) dans la traduction anonyme, conservée entre autres dans le manuscrit Paris, B.n.F. lat. 16204 (c. 1250) ${ }^{94}$. Aux pages 433a-500a, on peut lire le texte sous les titres suivants ${ }^{95}$ : Introductorius ad scienciam iudiciorum astrorum in interrogacionibus, De .L. preceptis, De interrogacionibus qui dicitur liber iudiciorum arabum, De significatore temporis in interrogacionibus, Liber electionum. Certains passages d'ordre assez général présents au livre I seulement (appelé Introductorius) sont indéniablement à rapprocher de citations du chapitre 9 du livre VIII du DPR (de zodiaco), où Barthélemy cite précisément « Misael » ou répète « idem auctor »; en outre, Barthélemy y fait référence à une « figura Misaelis » que nous ne retrouvons toutefois pas dans les manuscrits de la traduction latine de Zael. Nous avons comparé ces citations avec des éditions incunables de l'Introductorius, qui présentent un texte par endroits éloigné de celui que conservent les manuscrits du XIII ${ }^{\mathrm{e}}$ siècle ${ }^{96}$. Il apparaît que Barthélemy a dû passer par un intermédiaire qui reste indéterminé pour connaître Misael-Zael. La tradition parallèle nous permet cependant de lancer quelques pistes.

Les citations attribuées à Misael par Barthélemy dans les chapitres 9 (Zodiaque) et 10 (signes) présentent de fortes similitudes avec le Liber iudiciorum de Raymond de Marseille (écrit en 1141), qui a fondé sa compilation en deux parties sur le traité d'introduction à l'astrologie de Zael et sur les Iudicia attribués à Ptolémée ${ }^{97}$. Un manuscrit, que nous avons identifié comme un nouveau témoin du traité de Raymond de Marseille, fait un rapprochement intéressant entre le nom de Messahalla et celui de Zael, tout en ignorant le nom de Raymond. Il s'agit du codex München, B.S.B. clm 5198, f. 61r-69r, daté du dernier quart du XVe siècle, qui porte le titre : Incipit liber Messahala iudiciorum astrorum utilissimus quem dicit Cammeracensis fore Introductorium Messahala dicti Zahell. De la même façon, il serait envisageable que la forme Misael 
attestée chez Barthélemy soit le résultat d'une déformation graphique de Messahalla, que Pierre d'Ailly (Cameracensis) identifiait à Zael.

Le nom de Messahalla présent dans la liste d'autorités figurant à la fin du DPR n'est pas exempt de variations de graphie. Dans cette liste, on le trouve sous la forme Miselat astrologus, alors qu'il est cité dans le texte du livre VIII sous la forme Mischalach, en concurrence avec des citations différentes mises sous le nom de Misael. Quant au Liber iudiciorum de Raymond de Marseille, il évoque explicitement tantôt Zael, tantôt Messahalla au titre de source de tel ou tel chapitre sur les iudicia ${ }^{99}$. En conséquence, on peut supposer que la confusion entre Zael et Messahalla n'était pas du fait de Raymond de Marseille, mais qu'elle s'est opérée au fil de la transmission des textes.

Les Iudicia Ptolomei et le Liber iudiciorum de Raymond de Marseille ont été compilés par plusieurs auteurs tout au long du Moyen Âge, ce qui rend difficile l'identification de l'état primitif. Dans le tableau ci-dessous, nous avons comparé les caractéristiques physiognomoniques du visage du natif, induites par les signes du Zodiaque, avec les passages de ce type qui se trouvent dans le chapitre 10 du livre VIII de Barthélemy l'Anglais, dans le Liber astronomicus de Guido Bonatti, astrologue de la fin du XIII ${ }^{\mathrm{e}}$ siècle ${ }^{100}$, et dans le De figura seu imagine mundi de l'aragonais Louis de l'Angle (qui a effectué à Lyon des traductions de traités astrologiques dans les années 1440 et dédié cette œuvre au roi René d'Anjou en 1456). On constate que le texte de Barthélemy est plus proche de celui de Raymond de Marseille que de celui des Iudicia Ptolomei :

\begin{tabular}{|c|c|c|c|c|}
\hline $\begin{array}{l}\text { Iudicia Ptolomei } \\
\text { (London, B.L. Harley } \\
5402 \text { ) }\end{array}$ & $\begin{array}{l}\text { Raymond de } \\
\text { Marseille } \\
\text { (München, B.S.B. } \\
\text { clm 51, Paris B.n.F. } \\
\text { lat. 16208) }\end{array}$ & \begin{tabular}{|l} 
Barthélemy \\
l'Anglais, DPR \\
VIII.10
\end{tabular} & $\begin{array}{l}\text { Guido Bonatti, } \\
\text { De astronomia, } \\
\text { tract. III, pars I, } \\
\text { cap. 12, f. } 58 \mathrm{v}\end{array}$ & $\begin{array}{l}\text { Louis de l'Angle } \\
\text { (Paris B.n.F. lat. } 6561 \\
\text { St-Gall, Vadianiche } \\
\text { Sammlung, 427) }\end{array}$ \\
\hline $\begin{array}{l}\text { Aries itaque multos } \\
\text { capillos, curvum } \\
\text { corpus, faciem } \\
\text { longam habet, oculi } \\
\text { sunt graves ei, aures } \\
\text { parvas habet, longo } \\
\text { collo non indiget. }\end{array}$ & $\begin{array}{l}\text { Aries multos facit } \\
\text { capillos, corpus vero } \\
\text { curvum, corpus } \\
\text { oblongum, oculos } \\
\text { quandoque graves et } \\
\text { aures mediocres, } \\
\text { collum longum. }\end{array}$ & $\begin{array}{l}\text { [Misael] Aries ... } \\
\text { multos facit in } \\
\text { corpore capillos, } \\
\text { corpus curvum, } \\
\text { faciem obliquam, } \\
\text { oculos graves, } \\
\text { aures breves, } \\
\text { collum longum. }\end{array}$ & $\begin{array}{l}\text { Aries facit } \\
\text { hominem } \\
\text { habentem collum } \\
\text { longum et faciem } \\
\text { longam, multos } \\
\text { capillos, oculos } \\
\text { interdum graves, } \\
\text { aures parvas et } \\
\text { corpus ut multum } \\
\text { curvum. }\end{array}$ & $\begin{array}{l}\text { Aries ... Natus sub hoc } \\
\text { signo habet multos } \\
\text { capillos in corpore, } \\
\text { corpus curvum, faciem } \\
\text { obliquam, oculos } \\
\text { graves, aures breves, } \\
\text { collum longum. }\end{array}$ \\
\hline
\end{tabular}




\begin{tabular}{|c|c|c|c|c|}
\hline $\begin{array}{l}\text { Taurus frontem } \\
\text { amplam habet et } \\
\text { curvam semper et si } \\
\text { ampla non sit, } \\
\text { longum nasum gerit, } \\
\text { nares ample et oculi } \\
\text { graves sunt ei, } \\
\text { capilli elevati, } \\
\text { collum crassum. } \\
\text { Capillos nigros gerit. } \\
\text { Verecundus terram } \\
\text { inspicit. } \\
\text { honeste, Vadit } \\
\text { verumtamen vanus. }\end{array}$ & $\begin{array}{l}\text { Taurus tribuit } \\
\text { amplam faciem et } \\
\text { curvam, et si non } \\
\text { amplam dat nasum } \\
\text { grandem et longum, } \\
\text { nares amplos, oculos } \\
\text { graves, capillos } \\
\text { irsutos vel aliter } \\
\text { elevatos, collum } \\
\text { grossum et pingue, } \\
\text { capillos nigros, } \\
\text { verecundum } \\
\text { hominem reddit, } \\
\text { terram } \\
\text { prospicientem, } \\
\text { honeste incedentem, } \\
\text { tamen vanum. }\end{array}$ & $\begin{array}{l}\text { [Misael] Taurus } \\
\ldots \quad \text { dat faciem } \\
\text { amplam } \\
\text { curvam, nasum } \\
\text { dat gravem et } \\
\text { longum, amplas } \\
\text { nares, oculos } \\
\text { graves, capillos } \\
\text { elevatos et nigros, } \\
\text { collum grossum. } \\
\text { Verecundum } \\
\text { efficit hominem } \\
\text { honeste } \\
\text { incedentem, et } \\
\text { tamen vanum. }\end{array}$ & $\begin{array}{l}\text { Taurus facit } \\
\text { hominem } \\
\text { habentem faciem } \\
\text { amplam, } \\
\text { quandoque } \\
\text { curvam et si non } \\
\text { habuerit faciem } \\
\text { amplam, habebit } \\
\text { nasum grandem } \\
\text { et si non habebit } \\
\text { naturaliter } \\
\text { grandem, tractu } \\
\text { tamen temporis } \\
\text { habebit r per } \\
\text { accidens, nares } \\
\text { longas, oculos } \\
\text { nigros et graves, } \\
\text { capillos hirsutos } \\
\text { seu elevatos, } \\
\text { collum grossum } \\
\text { et pinguem, quasi } \\
\text { plus quam deceat, } \\
\text { verecundum, qui } \\
\text { terram prospicit } \\
\text { in eundo, honeste } \\
\text { incedentem, non } \\
\text { stabilem quasi } \\
\text { vagum, sed non } \\
\text { credas ei uxorem } \\
\text { tuam in secreto. }\end{array}$ & $\begin{array}{l}\text { Taurus ... Natus sub } \\
\text { hoc signo habet faciem } \\
\text { amplam, nasum } \\
\text { curvum et gravem et } \\
\text { longum, amplas nares, } \\
\text { oculos graves, capillos } \\
\text { elevatos et nigros, } \\
\text { collum grossum. } \\
\text { Verecundus est et } \\
\text { honeste incedens. }\end{array}$ \\
\hline $\begin{array}{l}\text { Gemini nec longi } \\
\text { nec curti sunt, sed } \\
\text { mediocrem gerunt } \\
\text { figuram, pectora } \\
\text { ampla, donant } \\
\text { pulcre persone, } \\
<\text { leviter> credens et } \\
\text { si Mercurius } \\
\text { prosperatur, erit } \\
\text { scriptor. }\end{array}$ & $\begin{array}{l}\text { Gemini mediocris } \\
\text { stature hominem } \\
\text { facit, pectora dat } \\
\text { ampla, pulcram et } \\
\text { convenientem } \\
\text { personam. Quod si } \\
\text { Mercurius dominus } \\
\text { Orientis tunc fuerit, } \\
\text { tunc fortunatus erit, } \\
\text { scriba nascitur. }\end{array}$ & $\begin{array}{l}\text { [Misael] Gemini } \\
\ldots \quad \text { mediocrem } \\
\text { efficit staturam, } \\
\text { hominem } \\
\text { pulchrum et } \\
\text { convenientem. Cui } \\
\text { coniunctus } \\
\text { Mercurius, } \\
\text { fuerit fortunatus, } \\
\text { disponit hominem } \\
\text { et habilem facit ad } \\
\text { literaturam et } \\
\text { scripturam. }\end{array}$ & $\begin{array}{l}\text { Gemini facit } \\
\text { hominem } \\
\text { habentem } \\
\text { mediocrem } \\
\text { staturam, pectus } \\
\text { amplum, } \\
\text { personam } \\
\text { convenientem. }\end{array}$ & $\begin{array}{l}\text { Gemini ... Natus sub } \\
\text { isto signo est } \\
\text { mediocris stature, } \\
\text { persona conveniens et } \\
\text { pulchra, hylaris est et } \\
\text { litteratus. }\end{array}$ \\
\hline
\end{tabular}




\begin{tabular}{|c|c|c|c|c|}
\hline $\begin{array}{l}\text { Cancer corpus habet } \\
\text { crossum, grossa est } \\
\text { ei pellis corporis, a } \\
\text { medietate superius } \\
\text { subtilis, dentes } \\
\text { torti; parvi sunt ei } \\
\text { oculi. }\end{array}$ & $\begin{array}{l}\text { Cancer dat corpus } \\
\text { haberer grossum } \\
\text { totiusque corporis } \\
\text { pellem grossam; a } \\
\text { medio inferius facit } \\
\text { hominem grossum } \\
\text { et incompositum, } \\
\text { superius rero } \\
\text { subtilem, [texte rayé } \\
\text { qui constituait les } \\
\text { lignes afférentes au } \\
\text { Lion et à la Vierge] } \\
\text { dentes curvos, tortos } \\
\text { modicos oculos. }\end{array}$ & $\begin{array}{l}\text { [pas de } \\
\text { marqueur] } \\
\text { Cancer ... facit in } \\
\text { corpore } \\
\text { grossitiem, nam a } \\
\text { medio eius facit } \\
\text { hominem r } \\
\text { grossum, r } \\
\text { incompositum } \\
\text { atque rudem, } \\
\text { superius vero } \\
\text { subtilem, dentes } \\
\text { curtos et tortos. }\end{array}$ & $\begin{array}{lr}\text { Cancer } & \text { facit } \\
\text { hominem } & \\
\text { habentem } & \text { corpus } \\
\text { incompositum et } \\
\text { cutim r totius } \\
\text { corporis } & \text { grossam } \\
\text { et } & \text { reddit } \\
\text { hominem r } & \\
\text { superius r } & \\
\text { subtilem, } & \text { inferius } \\
\text { grossum, } & \\
\text { habentem } & \text { dentes } \\
\text { tortos, r oculos } \\
\text { parvos. }\end{array}$ & $\begin{array}{lr}\text { Natus sub } & \text { Cancro } \\
\text { generaliter a } & \text { medio } \\
\text { corporis eius } & \text { est } \\
\text { grossus } & \text { et } \\
\text { incompositus atque } \\
\text { rudis, superbus tamen } \\
\text { et subtilis, habens } \\
\text { dentes tortos. }\end{array}$ \\
\hline $\begin{array}{l}\text { Leo ergo ab omnibus } \\
\text { cognoscitur, a } \\
\text { medietate superius } \\
\text { est grossus, a } \\
\text { medietate inferius } \\
\text { subtilis, magne est } \\
\text { voluntatis et crura } \\
\text { habet subtilia, id est } \\
\text { longa. }\end{array}$ & 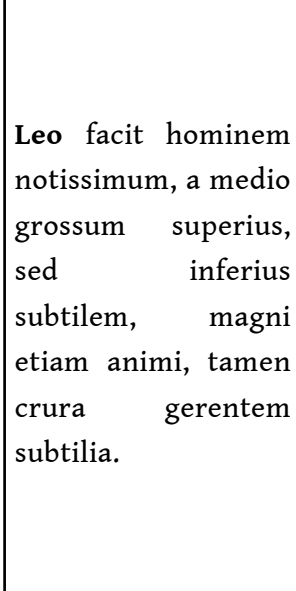 & 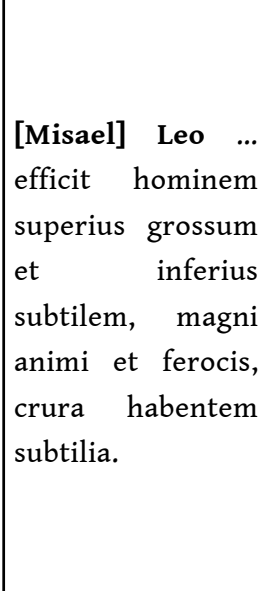 & $\begin{array}{l}\text { Leo facit } \\
\text { hominem } \\
\text { industrem, et } \\
\text { notum valde } \\
\text { atque } \\
\text { intelligentem, } \\
\text { superius } \\
\text { grossum, inferius } \\
\text { subtilem, nec } \\
\text { efficitur inde } \\
\text { indecens, } \\
\text { magnanimum, } \\
\text { crura subtilia. }\end{array}$ & $\begin{array}{l}\text { Leo ... generaliter est } \\
\text { superius grossus et } \\
\text { inferius subtilis, magni } \\
\text { animi et ferocis, crura } \\
\text { habet subtilia. }\end{array}$ \\
\hline $\begin{array}{l}\text { Virgo pulcram } \\
\text { personam habet, est } \\
\text { ipsa quoque bone } \\
\text { voluntatis, pulcri } \\
\text { inspiciuntur } \\
\text { occuli, ei faciem } \\
\text { pulcram habet. }\end{array}$ & $\begin{array}{l}\text { Virgo pulcram dat } \\
\text { personam bonamque } \\
\text { voluntatem, } \\
\text { pulchros oculos } \\
\text { faciemque } \\
\text { decentem. }\end{array}$ & \begin{tabular}{|lr} 
[pas r & de \\
marqueur] & Virgo \\
.. & personam \\
pulchram & facit, \\
pulchros & oculos \\
faciemque & \\
decoram, r & et \\
mitigat & \\
voluntatem.
\end{tabular} & $\begin{array}{l}\text { Virgo facit } \\
\text { hominem } \\
\text { habentem } \\
\text { pulchram } \\
\text { personam et } \\
\text { bonam } \\
\text { voluntatem, } \\
\text { pulchros oculos } \\
\text { et raciem } \\
\text { decentem. }\end{array}$ & $\begin{array}{l}\text { Virgo ... generaliter } \\
\text { erit persona pulcra et } \\
\text { pulcros habebit oculos } \\
\text { et faciem decoram et } \\
\text { mitigabit voluntatem. }\end{array}$ \\
\hline $\begin{array}{l}\text { Libra pulcra facies } \\
\text { est ei, caro illi } \\
\text { mediocris et est } \\
\text { amatrix mulierum. }\end{array}$ & $\begin{array}{l}\text { Libra convenientem } \\
\text { tribuit faciem, } \\
\text { carnem mediocrem, } \\
\text { mulierum constituit } \\
\text { amatorem. }\end{array}$ & $\begin{array}{l}\text { Libra } \\
\text { [description } \\
\text { absente] }\end{array}$ & $\begin{array}{l}\text { Libra facit } \\
\text { hominem } \\
\text { habentem faciem } \\
\text { convenientem, } \\
\text { mediocriter } \\
\text { carnosum, qui } \\
\text { etiam mulieres } \\
\text { amabit. }\end{array}$ & \begin{tabular}{ll} 
Libra $\quad .$. & \multicolumn{2}{c}{ hanchas } \\
habebit grossas et \\
renes debiles. Et est \\
amator r mulierum \\
naturaliter.
\end{tabular} \\
\hline
\end{tabular}




\begin{tabular}{|c|c|c|c|c|}
\hline $\begin{array}{l}\text { Scorpius facies est } \\
\text { ei rubicunda, multi } \\
\text { capilli, parvi sunt ei } \\
\text { oculi, faciem parvam } \\
\text { habens, crura longa } \\
\text { gerens, magni sunt } \\
\text { ei pedes. Leviter } \\
\text { movent, mendacium } \\
\text { ori eius non deest, } \\
\text { irascitur cum } \\
\text { omnibus leviter. }\end{array}$ & 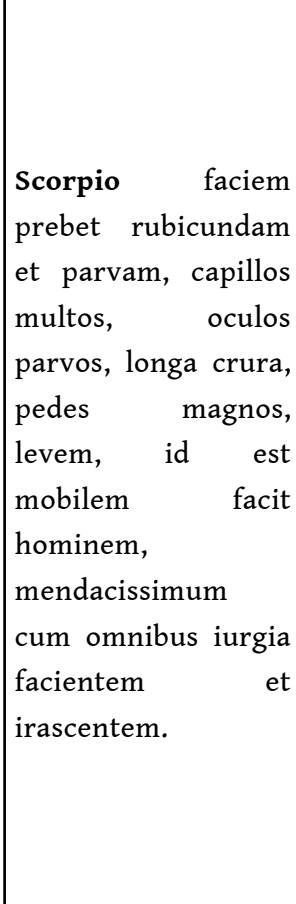 & $\begin{array}{l}\text { [Misael] Scorpius } \\
\ldots \quad \text { faciem dat } \\
\text { rubicundam et } \\
\text { parvam, capillos } \\
\text { multos, oculos } \\
\text { parvos, crura } \\
\text { longa, pedes } \\
\text { magnos et levem } \\
\text { facit hominem et } \\
\text { instabilem, } \\
\text { irascentem } \\
\text { mendacem. }\end{array}$ & $\begin{array}{l}\text { Scorpio facit } \\
\text { hominem } \\
\text { habentem faciem } \\
\text { rubicundam et } \\
\text { parvam, multos } \\
\text { capillos, oculos } \\
\text { parvos, longa } \\
\text { crura, pedes } \\
\text { magnos, cito } \\
\text { mutabilem, in } \\
\text { cuius ore vix aut } \\
\text { nunquam } \\
\text { invenietur } \\
\text { veritas, } \\
\text { irascentem, } \\
\text { litigatorem } \\
\text { cum ot } \\
\text { litem et rixam et } \\
\text { quasi pro nihilo } \\
\text { facientem. }\end{array}$ & $\begin{array}{lr}\text { Scorpio ... } & \text { Natus sub } \\
\text { eo habet } & \text { faciem } \\
\text { rubicundam } & \text { et } \\
\text { parvam, r capillos } \\
\text { multos et oculos } \\
\text { parvos, crura longa, } \\
\text { magnos pedes, levis } \\
\text { erit et instabilis, } \\
\text { irascens et mendax. }\end{array}$ \\
\hline $\begin{array}{l}\text { Sagittarius longas } \\
\text { coxas, crura habet } \\
\text { grossa, longam } \\
\text { faciem, milia faciei } \\
\text { ampla, mentum } \\
\text { subtile habet et } \\
\text { pulcrior retro quam } \\
\text { ante est, capillos } \\
\text { subtiles habet, } \\
\text { ventrem habet } \\
\text { grandem, equitare } \\
\text { amat. }\end{array}$ & $\begin{array}{l}\text { Sagittarius longas } \\
\text { facit coxas et crura, } \\
\text { faciem oblongam, } \\
\text { mila faciei } r \text { ampla } \\
\text { mentumque } \\
\text { a tergtile, } \\
\text { aliquem } \\
\text { pulchriorem quam } \\
\text { ab antecedentibus } \\
\text { esse, capillos dat } \\
\text { subtiles, ventrem } \\
\text { quandoque r } \\
\text { magnum, r in } \\
\text { equitando r facit } \\
\text { hominem delectari. }\end{array}$ & $\begin{array}{lr}\text { [Misael] } \\
\text { Sagittarius } \\
\text { longas facit coxas } \\
\text { et crura, faciem } \\
\text { oblongam r et } \\
\text { mentum subtile, } \\
\text { hominem } r \text { facit } \\
\text { pulchriorem retro } \\
\text { quam r ante, } \\
\text { capillos rat } \\
\text { subtiles, ventrem } \\
\text { magnum et in } \\
\text { motu r facit } \\
\text { hominem rr } \\
\text { delectari. }\end{array}$ & $\begin{array}{l}\text { Sagittarius facit } \\
\text { hominem } \\
\text { habentem longas } \\
\text { coxas et longam } \\
\text { faciem et milam } \\
\text { faciei amplam, } \\
\text { mentum subtile } \\
\text { et ut multum } \\
\text { pulchriorem a } \\
\text { tergo r quam } \\
\text { anterius, capillos } \\
\text { subtiles r et } \\
\text { aliquando r } \\
\text { ventrem maiorem } \\
\text { debito r et } \\
\text { delectatur } \\
\text { equitando. }\end{array}$ & $\begin{array}{l}\text { Sagittarius ... Natus } \\
\text { sub eo habet longas } \\
\text { coxas et crura, faciem } \\
\text { oblongam, caput } \\
\text { subtile, pulchrior est } \\
\text { retro quam ante, } \\
\text { capillos habet subtiles, } \\
\text { ventrem magnum et in } \\
\text { motu delectatur } \\
\text { naturaliter. }\end{array}$ \\
\hline $\begin{array}{l}\text { Capricornus subtilia } \\
\text { crura habet, siccum } \\
\text { corpus, facies similis } \\
\text { hirco, capillos } \\
\text { multos habet. }\end{array}$ & $\begin{array}{lr}\text { Capricornus subtilia } \\
\text { prebet crura, corpus } \\
\text { veror siccum } \\
\text { faciemque } \\
\text { multosque capillos. }\end{array}$ & $\begin{array}{l}\text { [Misael] } \\
\text { Capricornus } \\
\text { subtilia dat crura, } \\
\text { corpus siccum, } \\
\text { faciem hispidam } \\
\text { et irsutam. }\end{array}$ & $\begin{array}{l}\text { Capricornus facit } \\
\text { hominem } \\
\text { habentem crura } \\
\text { subtilia, corpus } \\
\text { siccum, faciem } \\
\text { hircinam, multos } \\
\text { capillos. }\end{array}$ & $\begin{array}{l}\text { Capricornus ... Natus } \\
\text { sub eo habet crura } \\
\text { subtilia, corpus siccum } \\
\text { et faciem, hispidus est } \\
\text { et hirsutus. }\end{array}$ \\
\hline
\end{tabular}




\begin{tabular}{|c|c|c|c|c|}
\hline $\begin{array}{l}\text { Aquarius tenet se } \\
\text { altum nimis est } \\
\text { pulcre artis, satis } \\
\text { expendit atque } \\
\text { perdit et devastat, } \\
\text { unus crus grossior } \\
\text { altero est, facies ei } \\
\text { pulcra et colorata. }\end{array}$ & 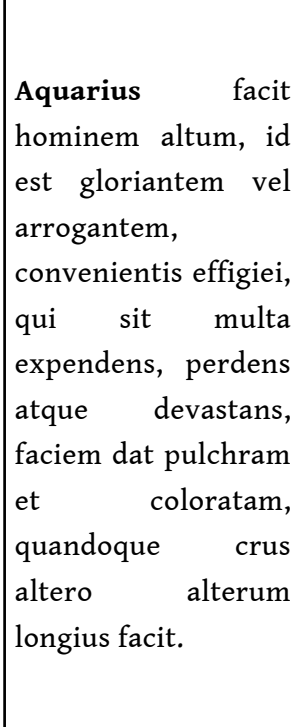 & $\begin{array}{l}\text { [Misael] } \\
\text { Aquarius ... } \\
\text { hominem } \\
\text { gloriantem, multa } \\
\text { devastantem, } \\
\text { pulchram racit } \\
\text { faciem } \\
\text { coloratam } \\
\text { tibiam } r \\
\text { alicuius } \\
\text { longiorem. }\end{array}$ & $\begin{array}{l}\text { Aquarius facit } \\
\text { hominem altum } \\
\text { et vana gloriosum } \\
\text { et arrogantem, } \\
\text { qui etiam erit } \\
\text { magnus expositor } \\
\text { et multum fit } \\
\text { prodigus r et } \\
\text { devastator } \\
\text { bonorum, } \\
\text { pulchram } \\
\text { habentem faciem } \\
\text { et coloratam et } \\
\text { aliquando unum } \\
\text { crus longius } \\
\text { altero. }\end{array}$ & $\begin{array}{l}\text { Aquarius ... Natus sub } \\
\text { isto signo est glorians, } \\
\text { multa devastans, } \\
\text { pulchram habebit } \\
\text { faciem et coloratam et } \\
\text { tibiam habebit altera } \\
\text { longiorem. }\end{array}$ \\
\hline $\begin{array}{l}\text { Pisces amplum } \\
\text { pectus habent, } \\
\text { parvum caput inest, } \\
\text { pulcra barba floret, } \\
\text { milia faciei sunt } \\
\text { grandia, albus est, } \\
\text { magne similiter } \\
\text { voluntatis, oculos } \\
\text { gerit ipse rotundos. }\end{array}$ & $\begin{array}{l}\text { Pisces amplum dat } \\
\text { pectus parvumque } \\
\text { caput, florentem } \\
\text { barbam, mila faciei } \\
\text { ampla, album facit } \\
\text { hominem, magni } \\
\text { animi, rotundis } \\
\text { oculis. }\end{array}$ & $\begin{array}{lr}\text { [Misael] } & \text { Pisces ... } \\
\text { amplum r } & \text { dat } \\
\text { pectus, parvum } \\
\text { caput, florentem } \\
\text { barbam r et } \\
\text { pulchram, album } \\
\text { facit hominem, } \\
\text { magni } \\
\text { rotundisque } \\
\text { oculis. }\end{array}$ & 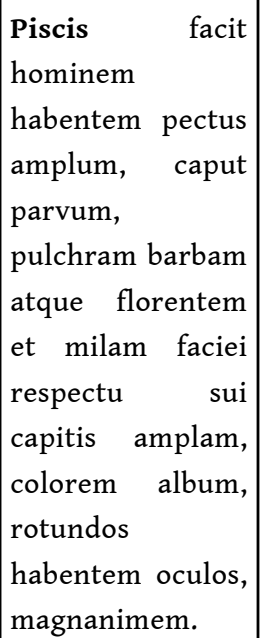 & $\begin{array}{l}\text { Pisces ... Natus sub } \\
\text { Piscibus habet amplum } \\
\text { pectus, parvum caput, } \\
\text { florens barbam, } \\
\text { pulchram, albus est et } \\
\text { magni animi } \\
\text { rotundosque habet } \\
\text { oculos. }\end{array}$ \\
\hline
\end{tabular}

\section{Les divers ludicia Ptolomei via des emprunts à Arnold de Saxe et peut-être à Raymond de Marseille}

En-dehors des citations du chapitre 10 attribuées à Misael et comparables à un type de Iudicia attribués à Ptolémée, d'autres citations sont mises par Barthélemy sous le marqueur Ptolomeus in libro de iudiciis astrorum. Elles sont à diviser en deux groupes. Le premier peut être rapproché d'un bref traité De motibus et iudiciis planetarum (inc. Sub Saturno sunt hec signa Capricornus et Aquarius et sunt eius domus) auquel nous avons consacré un article où sont mises en évidence quelques voies de sa réception jusqu'à la fin du Moyen Âge. Présents dans huit citations chez Barthélemy, ces iudicia ont pu être empruntés à Arnold de Saxe (son De floribus rerum naturalium les conserve en sept passages référencés sous le titre De motibus et iudiciis planetarum). Ils se retrouvent aussi dans des miscellanées astrologiques du $\mathrm{XV}^{\mathrm{e}}$ siècle réparties en deux traditions, l'une contenant le De signis et imaginibus celi de Michel Scot, l'autre une partie du livre III du De astronomia d'Hygin. Nous avons donné une édition critique de ce court texte relatif aux significations caractérielles (colère, sévérité, tristesse, richesse...), aux couleurs et aux qualités (chaude, humide...) des planètes ainsi qu'aux signes de leur exaltation et de leur 
chute ${ }^{101}$. Comme nous l'avons indiqué plus haut, une version de ce texte était connue par l'Experimentator également.

Le deuxième groupe de citations attribuées par Barthélemy à un De iudiciis Ptolomei contribue à la description des planètes (DPR, VIII.12-18). Il s'agit des Iudicia Ptolomei (Ms. London, British Libr., Harley 5402, f. 2r-2v), aussi appelés Iudicia ad Aristonem ou Liber iudiciorum - Liber ad Alconem regem - Doctrina Ptolomei data filio suo, inc. : Signorum alia sunt masculini generis, alia feminini. Feminini, ut Taurus. Ce texte a été utilisé par Raymond de Marseille dans son Liber iudiciorum, et c'est précisément de la version de Raymond de Marseille que les extraits cités par Barthélemy se rapprochent le plus. Un autre traité de iudicia attribué tant à Ptolémée qu'à Aristote (inc. : Signorum alia sunt masculini generis, alia feminini. Masculini generis sunt ista : Aries, Gemini, Ms. Paris, B.n.F., lat. 16208, f. 76ra-83vb) remonte au plus tard au XII siècle $^{102}$. Les similitudes entre ces versions des iudicia et la compilation de Barthélemy apparaissent dans le tableau ci-dessous ${ }^{103}$.

\begin{tabular}{|c|c|c|c|}
\hline $\begin{array}{l}\text { Pseudo-Ptolémée, } \\
\text { Iudicia, London, B.L. } \\
\text { Harley 5402, f. 2v-3r }\end{array}$ & $\begin{array}{l}\text { Pseudo-Aristote, } \\
\text { Iudicia, Paris, B.n.F., } \\
\text { lat. 16208, f. 77vb }\end{array}$ & $\begin{array}{lr}\text { Raymond } & \text { de } \\
\text { Marseille, } & \text { Liber } \\
\text { iudiciorum, } & \text { Paris, } \\
\text { B.n.F., lat. } & 16208, \\
\text { f. 13rb } & \end{array}$ & $\begin{array}{l}\text { Barthélemy } \\
\text { l'Anglais, } \\
\text { VIII.12 }\end{array}$ \\
\hline $\begin{array}{l}\text { Saturnus itaque est } \\
\text { homo fuscus, } \\
\text { paucos pilos in barba } \\
\text { habet, non est } \\
\text { pulcher, } \\
\text { operatur iniqua, } \\
\text { gravis, piger, } \\
\text { non ridet } \\
\text { et ragadias quod sepe } \\
\text { habet in calcaneo, } \\
\text { color est glaucus, } \\
\text { pectus quoque inest } \\
\text { ei subtile, } \\
\text { capilli sunt ei asperi, } \\
\text { fetida sunt ei } \\
\text { vestimenta et nigra } \\
\text { libentius induit } \\
\text { et similis est habenti } \\
\text { malam voluntatem }\end{array}$ & $\begin{array}{l}\text { Saturnus itaque est } \\
\text { homo fuscus, id est nec } \\
\text { albus nec niger, sed } \\
\text { glaucus, } \\
\text { operatur numquam, } \\
\text { non ridet } \\
\text { et si vadit discalciatus } \\
\text { habet scissuras sepe in } \\
\text { calcaneo, nigra } \\
\text { libentius induit et } \\
\text { similis habenti malam } \\
\text { voluntatem (generalia } \\
\text { sunt); } \\
\text { paucos pilos habet in } \\
\text { barba, non est pulcer, } \\
\text { non ridet, pectus inest } \\
\text { ei subtile, } \\
\text { capilli sui sunt asperi, } \\
\text { vestimenta fetida sunt } \\
\text { ei, gravis, piger } \\
\text { (particularia sunt). }\end{array}$ & 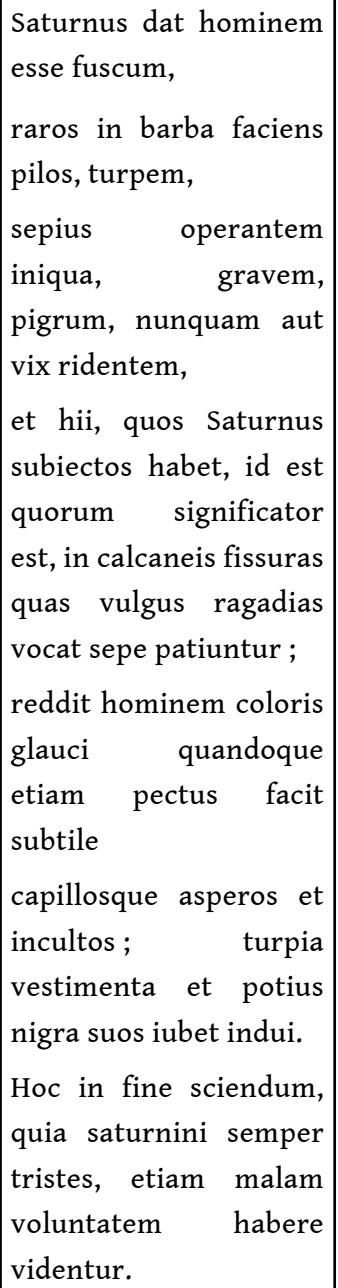 & $\begin{array}{l}\text { Saturnus [...] dat } \\
\text { hominem } \\
\text { fuscum, turpem, } \\
\text { iniqua operantem, } \\
\text { pigrum, gravem, } \\
\text { tristem, raro hilarem } \\
\text { seu ridentem. } \\
\text { Unde dicit idem } \\
\text { Ptolomeus: Subiecti } \\
\text { Saturno ragadias, id } \\
\text { est fissuras siccas, in } \\
\text { calcaneis sepius } \\
\text { patiuntur, } \\
\text { glauci sunt coloris et } \\
\text { lividi, } \\
\text { in capillis et in toto } \\
\text { corpore asperi et } \\
\text { inculti, turpia et } \\
\text { fetida non abhorrent } \\
\text { vestimenta, animalia } \\
\text { diligunt fetida et } \\
\text { immunda, } \\
\text { res diligunt acidas et } \\
\text { stipticas, quia in } \\
\text { eorum complexione } \\
\text { humor melancolicus } \\
\text { dominatur104. }\end{array}$ \\
\hline
\end{tabular}


69

comparaison nous permet de constater que Barthélemy a puisé la version des iudicia qu'il a compilée à une source très proche, sinon identique à celle utilisée par Raymond de Marseille. Cependant, les quelques phrases qui figurent chez Barthélemy à la fin de l'extrait et qui mettent en rapport la complexion mélancolique et l'influence de Saturne sont absentes des autres iudicia, ce qui nous conduit à conclure qu'il a eu accès à une version réécrite du traité de Raymond.

\section{Sources « arabes »}

Nous avons déjà souligné le fait que Barthélemy ne semble pas avoir utilisé les œuvres d'astronomes comme al-Bitrūjī et al-Battānī. Les citations qui sont associées en différents endroits aux noms d'Alfraganus (al-Farghānī) et d'Algazel (al-Ghazzāli) ont été importées via un intermédiaire que nous n'avons pas réussi à identifier. C'est aussi à travers des intermédiaires que certaines doctrines et principes généraux de l'astrologie arabe se retrouvent dans le livre VIII du DPR. Ainsi, nous avons pu établir une vingtaine de lieux parallèles avec l'Introductorius d'al-Qabīsī, qui constitue un des premiers textes arabes à avoir été traduits en latin en Espagne au XII ${ }^{\mathrm{e}}$ siècle, en l'occurrence par Jean de Séville avant $1135^{105}$. Parmi ces lieux parallèles, quatre se retrouvent aussi dans l'Introductorium de Zael (Sahl b. Bishr) qui fut par ailleurs une des sources d'al-Qabīsī, une douzaine d'autres étant communs avec l'Introductorius maior d'Albumasar (Abū Ma'shar).

71 Le nom d'Albumasar revient à plusieurs reprises dans le livre VIII. On compte au total 23 occurrences, dont trois sont explicitement associées à des titres: une au De motibus astrorum, une au De motibus planetarum et une autre au De iudiciis astrorum. Ces titres indiquent-ils des œuvres distinctes? La citation qui renvoie au De iudiciis astrorum et trois autres ont pu être repérées dans l'Introductorius maior. Les extraits qui sont attachés aux marqueurs De motibus astrorum et De motibus planetarum apparaissent dans la compilation d'Arnold de Saxe, où ils se trouvent sous une forme plus riche et plus correcte. La citation du De motibus planetarum vise à expliquer le phénomène des éclipses, alors que les extraits du De motibus astrorum concernent les étoiles fixes seulement; ces deux thèmes astronomiques pourraient renvoyer à des traités distincts ${ }^{106}$.

Le De motibus astrorum s'inscrit dans la longue tradition des textes astrométéorologiques ( parapegmata) qui mettent en rapport le lever et le coucher des constellations et les changements climatiques affectant la Terre. La nomenclature des constellations est tributaire autant de l'astronomie gréco-latine que de l'arabe. À la différence de tous les témoins de cette littérature étudiés entre autres par P. Kunitzsch, Ch. Burnett et D. Lehoux ${ }^{107}$, le texte attribué à Albumasar introduit une division des signes du zodiaque par 30 degrés destinée à situer l'apparition de telle ou telle constellation, division qui se substitue au calendrier mensuel utilisé communément. De ce fait, le texte ne présente plus les caractéristiques d'un calendrier et implique une lecture astrologique. Ce type de division par degrés, qui témoigne de l'importance prise par la notion de gradus dans la littérature astronomique latine à partir $\mathrm{du} \mathrm{X}^{\mathrm{e}}$ siècle, n'est que la conversion en degrés zodiacaux du calendrier mensuel, de telle sorte que le premier jour du mois est en général identifié au $17^{\mathrm{e}}$ ou $18^{\mathrm{e}}$ degré du signe zodiacal corrélatif. Voici quelques exemples de correspondances entre le parapegma (De mutatione aeris) intégré aux Iudicia Ptolomei et le De motibus astrorum compilé par Arnold de Saxe et, à travers ce dernier, par Barthélemy l'Anglais. 


\begin{tabular}{|c|c|c|}
\hline $\begin{array}{l}\text { Jour du mois (Iudicia } \\
\text { Ptolomei) }\end{array}$ & $\begin{array}{l}\text { Degré du signe (Arnold de } \\
\text { Saxe, DFRN) }\end{array}$ & $\begin{array}{l}\text { Constellation (Iudicia Ptolomei et } \\
\text { Arnold de Saxe DFRN) }\end{array}$ \\
\hline 1 septembre & $18^{\circ}$ Virginis & Icarus, custos plaustri \\
\hline 7 septembre & $23^{\circ}$ Virginis & Vespertinus \\
\hline 14 septembre & $30^{\circ}$ Virginis & Arcturus \\
\hline 19 septembre & $5^{\circ}$ Libre & Sichis \\
\hline 25 septembre & & Alferat \\
\hline 6 octobre & $22^{\circ}$ Libre & Stephanos, id est Corona \\
\hline 7 octobre & $23^{\circ}$ Libre & Erifi, id est Edi Vespertini \\
\hline 6 novembre & $18^{\circ}$ Scorpionis & Lapsidis, id est Lucidus \\
\hline 13 novembre & $30^{\circ}$ Scorpionis & Lira \\
\hline 21 novembre & $8^{\circ}$ Sagittarii & Yades \\
\hline 27 novembre & $13^{\circ}$ Sagittarii & Stephanos, id est Corona \\
\hline 1 décembre & $18^{\circ}$ Sagittarii & Cion, id est Canis \\
\hline 10 décembre & $27^{\circ}$ Sagittarii & Erifi, id est Edi \\
\hline 21 décembre & & $\begin{array}{l}\text { Ectos, id est Aquila, et Esion, id est } \\
\text { Eridanus }\end{array}$ \\
\hline 2 juin & $18^{\circ}$ Geminorum & Ectos, id est Aquila \\
\hline 9 juin & $25^{\circ}$ Geminorum & Vespertinus \\
\hline 28 août & & Antevendemor (Antevindemiator) \\
\hline
\end{tabular}

Les Iudicia Ptolomei constituent le texte avec lequel le De motibus astrorum partage le plus grand nombre d'astronymes de souche gréco-latine. Mais, à l'exception des constellations provenant de la tradition antique, le De motibus astrorum comporte une quantité importante d'astronymes connus grâce aux traductions faites de l'arabe à partir du $\mathrm{X}^{\mathrm{e}}$ siècle, comme alramech, alfeka, aldebaran, calbalazeda, alferat, alrif. Les traités sur l'astrolabe ${ }^{108}$ et les nombreuses tables d'étoiles ${ }^{109}$ attestent la connaissance de ces constellations. Pourtant, aucun des textes astronomiques que nous avons consultés n'offre une liste complètement identique à celle du traité attribué chez Arnold de Saxe et Barthélemy à Albumasar. Une bonne partie des noms se retrouvent mentionnés dans les tables III et VIII de l'index de P. Kunitzsch, mais certaines constellations sont associées de façon assez curieuse aux signes du zodiaque. Ainsi, alramech et alchimech sont placées 
dans le signe du Bélier, alors que la littérature astronomique les rapporte au signe de la Balance.

L'auteur à l'origine de ce bref traité appelé « De motibus astrorum/ planetarum » par Arnold de Saxe et Barthélemy est probablement parti d'un texte similaire aux Iudicia Ptolomei, sur lequel il a greffé la nomenclature arabe de plusieurs constellations. Il est d'ailleurs possible que l'on ait cherché à insérer les astronymes arabes positionnés sur l'araignée des astrolabes dans le parapegma gréco-latin. La corruption de la graphie des arabismes dans les manuscrits d'Arnold de Saxe, que l'on constate pour les termes grecs et latins également, ne facilite d'ailleurs pas le repérage du modèle qui aurait pu servir à composer ce parapegma sans équivalent. Barthélemy l'Anglais, qui ne reprend les termes arabes que pour les constellations du signe du Bélier pour abandonner ensuite toute autre occurrence, retient principalement les constellations dont il traite à la fin du livre VIII, à savoir Arcturus, Orion, les Hyades, les Pléiades, la Canicule, les seules qu'Isidore avait lui aussi retenues dans ses Étymologies.

\section{E. Le milieu et la datation du livre VIII}

L'utilisation de diverses sources du De mundo que nous avons essayé de cerner dans ces pages, de même que les œuvres citées ailleurs par Barthélemy l'Anglais permettent de fixer un terminus a quo pour la rédaction du livre VIII du DPR. Ainsi, le prologue à l'Opus sententiarum d'Hugues de Saint-Cher rédigé vers 1236, les extraits sur la Trinité tirés du Liber extra publié en 1234, les citations littérales du De floribus rerum naturalium d'Arnold de Saxe, dont la collecte des sources doit être située entre 1225 et 1235, de l'Hexaëmeron de Robert Grosseteste, écrit entre 1228-1235, mais très probablement connu via la compilation de l'Experimentator, les passages de la Summa 'Alexandri de Hales', dont plusieurs parties étaient rédigées avant 1245, de la Questio de luce de Richard Fishacre, que l'on date des années 1245-1248, nécessitent de placer la rédaction du De mundo après cette dernière date. Par ailleurs, ces sources, connues dans le milieu parisien dès les années 1240, pourraient nous faire conclure que Barthélemy avait encore des liens avec Paris après son installation dans le couvent franciscain de Magdebourg en 1230 ou qu'il est retourné à Paris après y avoir séjourné quelques années. L'emprunt extensif de la glose anglicane du De vegetabilibus pseudo-aristotélicien dans le livre XVII vient compliquer les choses, puisqu'il a été dit jusqu'ici qu'elle n'était pas connue sur le continent, à moins de supposer qu'elle ait circulé dans les milieux anglais de Paris. Ces divers éléments fournissent des indices pour situer la chronologie relative de rédaction du DPR, dont il faut souligner que les livres diffèrent, tant par les sources utilisées, que par le moment de rédaction.

Le fait que Barthélemy exploite peu et de seconde main le corpus aristotélicien consacré à la physique céleste, qu'il ne connaisse directement ni le Liber introductorius de Michel Scot, ni ses traductions d'Averroès, qu'il n'ait pas une plus ample connaissance des écrits de Robert Grosseteste, relativise toute tentative de rattacher Barthélemy à un studium ou à un milieu universitaire. En attendant les conclusions des autres éditeurs du DPR, on ne peut que pointer vers le faisceau d'influences qui converge à partir des livres déjà édités. Cependant, si les auteurs-sources que nous avons identifiés peuvent être associés à des centres d'études comme Oxford, Paris et Magdebourg, cela ne signifie pas nécessairement que Barthélemy les a fréquentés assidûment ou qu'il n'a été actif qu'à ces endroits. Des compilateurs comme l'Experimentator et Arnold de Saxe ont été les véhicules d'une 
matière déjà rassemblée, qu'il a complétée afin de fournir une synthèse du savoir relatif aux réalités célestes. Cette connaissance, comme on va le voir, présentait des incohérences et des lacunes considérables qui ne font de Barthélemy ni un spécialiste ni un connaisseur.

\section{Savoir cosmologique et effets d'érudition ${ }^{110}$}

Les données astronomiques abordées dans le De mundo touchent pour la plupart à l'astrologie, c'est-à-dire à l'influence céleste sur le monde sublunaire. Astronomie et astrologie ne sont pas différenciées par Barthélemy, qui n'emprunte pas à Isidore de Séville la célèbre distinction entre connaissance des astres et divination, condamnant l'astrologie superstitieuse des mathematici qui « font même correspondre les douze signes célestes à chacune des parties du corps et de l'âme, et qui entreprennent de prédire la géniture et le caractère des hommes d'après le cours des étoiles ${ }^{111}$. À cet égard, Barthélemy l'Anglais adopte un ton neutre; il se limite à livrer des renseignements, annonçant que son but est d'expliciter les «noms » (nomina) des planètes afin de mieux comprendre l'Écriture sainte, alors que le contenu du livre VIII ne contient qu'une dizaine de références bibliques explicites ${ }^{112}$. L'action déterminante des astres sur la vie humaine ne constitue pas pour Barthélemy un sujet de controverse ou une thèse contre laquelle il faudrait prémunir les lecteurs, contrairement à certains de ses contemporains qui ont compilé les mêmes sources. Qu'il suffise de rappeler à ce propos les chapitres d'Hélinand de Froidmond sur les doctrines du Libellus de efficacia astrologiae d'Eudes de Champagne, où l'astrologue Zael est cité à plusieurs reprises ${ }^{113}$, la critique formulée par Robert Grosseteste dans l'Hexaëmeron, les mises en garde exprimées par Vincent de Beauvais dans le Speculum naturale (XV.50). Barthélemy bâtit son exposé en s'appuyant sur des autorités de l'astrologie grecque et arabe, évoquant fréquemment les influences bénéfiques ou maléfiques des planètes sur la terre, ou l'effet pernicieux de la Lune (dont il parle aussi dans le livre XVII du DPR sans citer les mêmes auctoritates).

Les sujets traités s'inscrivent dans un cadre cosmologique général où sont abordés des aspects relatifs à l'astrologie, à l'astro-météorologie, à la mélothésie, à la physiognomonie, à la médecine, mais qui ne portent pas, sauf exception, sur des questions d'astronomie et de physique céleste, telles que les distances entre planètes, la nature de leurs orbites, leur vitesse de déplacement, le vide dans le monde des sphères, l'existence d'une octava sphera.

L'ensemble du livre est structuré en trois parties : l'une dédiée à la nature des cieux, une deuxième à la description des planètes et des constellations et pour finir une section consacrée à la lumière. Le schéma des notices qui composent les chapitres de la deuxième partie paraît relativement répétitif. Les propriétés des signes du Zodiaque et des planètes sont systématisées selon les éléments suivants: une phrase de type étymologique, généralement attribuée à Isidore de Séville (sans que, le plus souvent, il s'agisse des Étymologies), un résumé astrologique portant sur les maisons, les triplicités et les faces, une éventuelle description physique de la planète, des considérations de type physiognomonique qui spécifient l'influence de telle ou telle composante céleste sur l'homme (mélothésie) et son caractère ou encore sur le développement du fœtus, des indices d'astro-météorologie. 
80 Les composantes de chaque étage cosmique ainsi que leurs relations entre elles exercent une influence, que ce soit sur une partie du ciel, pour les aspects (aspectus), ou sur la totalité des corps de l'univers, pour la lumière qui continet omnia corpora (DPR, VIII.29, 1.132). C'est l'ensemble de ces influences et les rapports entre le tout et les parties qui intéresse Barthélemy, car il a probablement trouvé dans l'astrologie les éléments aptes à constituer l'image d'un univers hiérarchisé, cohérent, homogène, interactif.

81 En construisant son exposé théorique, Barthélemy l'Anglais remplace l'ordre narratif du récit biblique par un ordre philosophique qui structure et hiérarchise la Création en fonction du degré de noblesse, de pureté de chaque composante. À cette hiérarchie cosmique qui donne sens à la narration philosophique répondent les degrés de la société ecclésiastique, souvent évoqués dans les notes marginales, dans un constant aller-retour entre le visible et l'invisible, le concret et l'abstrait, le corporel et l'incorporel. Autant le livre consacré aux anges que celui voué aux réalités célestes édifient ce type de hiérarchisation, tout en privilégiant la communication entre les différents étages, et dessinent une harmonie des opérations, déléguées d'un étage à l'autre. Ainsi, Barthélemy range les corps célestes à la suite des anges, de l'âme et de la nature de l'homme pour faire ensuite place aux réalités terrestres (habitat et habitants). Le livre astronomique comme celui dédié aux anges relèvent d'une vision dyonisienne, où la hiérarchie des créatures va de Dieu aux natures les plus obscures. Les hiérarchies des anges et des cieux, dont les domaines d'influence sont séparés, se complètent par les actions qui leur sont imparties: les anges «trans-portent » la lumière éternelle jusqu'à l'intellect humain (DPR, II.2, 1. 83-86); les planètes et les signes zodiacaux sont groupés en réseaux d'opération dotés d'effets sur la matière des corps (de l'homme et du reste des créatures) aussi bien que sur la nature de la terre toute entière, ce qui rend leurs propriétés interdépendantes. Avec la lumière, les entités célestes gouvernent les réalités inférieures, exercent leur influence sur la génération et la corruption, contribuent aux différentes étapes de la formation de l'embryon et du fotus. En revanche, les planètes ne sont plus dotées d'une âme comme elles l'étaient chez Macrobe par exemple. L'idée de l'anima mundi, si présente au XII siècle, disparait aussi chez Barthélemy, qui construit l'harmonie du monde par l'entremise de la doctrine de la participation : toutes les choses prennent part au dessein divin en fonction de leurs propriétés, selon le degré de lumière qu'elles comportent.

\section{A. Hiérarchies des cieux}

82 La synthèse que Barthélemy l'Anglais présente sous le titre neutre De mundo et corporibus celestibus est résumée par un renvoi inséré à la fin du prologue du livre $\mathrm{X}$ comme liber de bonitate mundi $i^{114}$. Cette deuxième appellation confère une portée théologique à l'exposé censé réunir les principaux éléments relatifs aux corps célestes et au cosmos.

La distinction platonicienne énoncée au premier chapitre, entre le caractère éternel du monde-archétype et le caractère perpétuel du monde sensible, préside de façon assez considérable à l'exposé des réalités célestes. Bien qu'il fasse mention, comme Arnold de Saxe, du titre De mundi eternitate cité en tête d'un chapitre du Liber celi et mundi de PseudoAvicenne (VIII.1, 1. 104), Barthélemy n'aborde jamais le thème de l'éternité du monde, pourtant déjà présent dans les débats de la scolastique naissante. Qu'il s'agisse de la nature du monde, du firmament ou de la lumière du Soleil, ce sont les termes appariés à perpetuus et sempiternus qui font office d'éléments qualificatifs ${ }^{115}$. Il n'est pourtant pas 
clair si cette temporalité affecte au même degré toutes les strates du cosmos, depuis la terre jusqu'à l'empyrée. Les 54 occurrences des adverbes signifiant un état ou un processus permanent (44 pour semper, 10 pour numquam), dont il faut soustraire les quelques formulations restrictives ( 7 fois non semper, 1 fois semper fere), renvoient à l'idée de régularité de certains phénomènes, manifestes surtout dans l'espace en-deçà du firmament. Elles n'évoquent toutefois pas la même idée de durée que les adjectifs perpetuus et sempiternus.

84 Le monde céleste, avec lequel Barthélemy entame l'exposé du monde sensible, est décrit de façon synthétique en tant que sphère, et de manière analytique comme succession de sphères dotées des res corrélatives. Des définitions mises sous l'autorité de Martianus Capella et d'Isidore de Séville expriment dans les mêmes termes le sens de mundus et de sphera, espace de forme sphérique ayant la terre pour centre ${ }^{116}$. La sphéricité est déduite d'un raisonnement métaphysique et théologique, car la sphère est censée être la forme la plus apte à contenir l'ensemble de la Création et la plus semblable à Dieu, car elle n'a ni début ni fin. Malgré ce rapprochement sémantique entre le monde et la sphère, d'autres données réunies au chapitre 6 identifient la sphère avec le ciel des étoiles fixes, le firmament, dont la vitesse de mouvement est contrecarrée par le mouvement des sept planètes.

C'est par rapport à cette surface sphérique que Barthélemy définit l'axe de rotation passant par les deux pôles, de même que les onze cercles célestes ${ }^{117}$, parmi lesquels deux cercles visibles, à savoir la Voie Lactée ${ }^{118}$ et le Zodiaque, et neufs autres invisibles : les cinq parallèles (l'équateur, le tropique du Cancer, le cercle arctique, le tropique du Capricorne et le cercle antarctique), les deux colures (solsticial et équinoxial), l'horizon (plus précisément l'horizon sensible) et le méridien. Au sujet de ces deux derniers cercles, Barthélemy, en suivant sa source ${ }^{119}$, tient à rappeler qu'ils ne sont pas figurés sur la sphère en raison de la position variable qu'ils ont selon le point d'observation ${ }^{120}$. Cette référence à la sphère armillaire remonte à Macrobe (Commentaire au Songe de Scipion, I.15.15); en la reprenant par le biais de l'Experimentator, Barthélemy savait-il de quel instrument il était question? Difficile de le dire, même si son usage est bien attesté pour le XIII ${ }^{\mathrm{e}}$ siècle. Toujours est-il que le point de vue de l'observateur, que traduisent les 43 références à notre position ici-bas (nos 8 occurrences, nobis 16, noster 19), structure l'exposé des réalités célestes, rangées globalement selon qu'elles sont visibles ou invisibles.

La différenciation des sphères entre elles réside surtout dans leur constitution matérielle, le degré auquel elles possèdent une matière simple, pure et, par là-même, noble. Cette représentation métaphysique est articulée sur une relation directement proportionnelle entre la pureté et la simplicité de la matière et sa disposition à revêtir une forme noble ; elle a pour fonction la classification de toute la création, des entités incorporelles jusqu'aux entités corporelles inanimées. Ainsi, en vertu de leur matière, les couches célestes et élémentaires sont plus ou moins aptes à obéir, comme le répète Robert Grosseteste, à la force motrice du firmament ${ }^{121}$. Densité et pesanteur rendent donc la Terre inapte à suivre le mouvement du firmament. Et, comme le rappelle ailleurs une citation de Pseudo-Avicenne, plus on s'éloigne de la source du mouvement, plus la chaleur et la mobilité diminuent ${ }^{122}$. Ces raisonnements s'appuyent sur la production du mouvement des sphères célestes et sa transmission de l'une à l'autre ainsi que sur la nature de la matière à la base de chacune des couches. Ainsi, une série de processus physiques explique l'immobilité de la Terre ; cela nuance la théorie qui faisait de la Terre 
une entité immobile comme seule condition physique apte à expliquer le mouvement de la sphère des fixes ${ }^{123}$.

Les cieux sont hiérarchisés selon plusieurs critères. Selon la tradition exégétique, ils sont sept, par oppostion aux théories des philosophes partisans du ciel unique ${ }^{124}$, rayées d'un trait moqueur par Basile de Césarée ${ }^{125}$. D'abord, une distinction globale sépare les cieux visibles (l'aérien, l'éthéré, l'olympien, l'igné et le firmament) des cieux invisibles (l'aqueux - ou cristallin - et l'empyrée). La diversité des interprétations patristiques réunies par Barthélemy n'attribue pas un statut nettement défini au ciel olympien et entraîne également une certaine confusion dans la définition du ciel éthéré et du ciel igné. L'éthéré est placé dans la proximité immédiate de la sphère du feu, alors que le ciel igné détient, lui, certaines propriétés nobles grâce au voisinage des sphères des planètes, qui ne sont rapportées à aucun ciel.

Selon Grégoire le Grand, dont le texte rapporté est glosé, l'éthéré s'étendrait de la Lune jusqu'au firmament et contiendrait les sphères des sept planètes ${ }^{126}$. Une référence ${ }^{127}$ renvoie le lecteur au chapitre 5, où Barthélemy abonde dans le sens de ce "Grégoire ». L'imbrication de citations extraites des Météorologiques d'Aristote, du Liber celi et mundi de Pseudo-Avicenne et du commentaire au De nuptiis Philologiae et Mercurii de Rémi d'Auxerre confère à l'éther le statut de substance distincte des quatre éléments, qui, par la vitesse de son mouvement, contrebalance celui du firmament et produit la sphère du feu. C'est dans l'éther que seraient contenues les sphères des sept planètes. En conséquence de cette théorie, l'ordre des cieux se trouverait réaménagé, car l'éthéré viendrait prendre place entre l'igné et le firmament.

Cependant, par l'entremise d'un développement emprunté à Alexandre de Halès, Barthélemy propose un ordre des cieux selon qu'ils sont source (dativum) ou réceptacle ( receptivum) de la lumière, selon qu'ils sont uniformes ou non, selon qu'ils sont mobiles ou non $^{128}$. Ainsi l'empyrée, qui est source première de la lumière, est uniforme et immobile ; le ciel cristallin est aussi source de lumière, uniforme, mais mobile ; le firmament est un ciel mobile et source de lumière, mais sans être uniforme ; l'olympien et l'igné, qui sont associés à l'élément feu, sont des réceptacles de la lumière, l'un la recevant sans production de chaleur (l'olympien), l'autre avec (l'igné). Les deux derniers étages, l'éthéré et l'aérien, qui relèvent de l'air, sont aussi des réceptacles de la lumière. Ils sont distingués selon qu'ils sont conjoints à la partie supérieure (l'éthéré) ou inférieure (l'aérien) de l'air. De l'ordre proposé par la Summa 'Alexandri', qui finit par intervertir les positions de l'olympien et de l'igné par rapport à la tradition exégétique, Barthélemy conclut que l'olympien est le ciel des sphères planétaires. Cette interversion est adoptée par Bonaventure ${ }^{129}$ également, qui choisit cependant d'ajouter le ciel des planètes, alors qu'Albert le Grand ${ }^{130}$, dont le raisonnement ressemble fortement à celui d'Alexandre de Halès, et Thomas d'Aquin ${ }^{131}$ vont suivre la hiérarchie des cieux héritée de Raban Maur et de la glose ordinaire.

Barthélemy répertorie donc trois dispositions des cieux et ne semble pas se prononcer pour l'une ou l'autre. Le parcours ascendant qu'il adopte pour son exposé en partant des cieux inférieurs pour traiter ensuite du firmament (DPR, VIII.2), du cristallin (DPR, VIII.3) et de l'empyrée (DPR, VIII.4) pour revenir, une fois de plus, à l'éther (DPR, VIII.5) laisserait croire que, dans l'économie des interactions célestes, il privilégie l'éther comme sphère des planètes. Cela permettait de dissocier le monde sublunaire, soumis à la génération et à la corruption, du monde supralunaire incorruptible et de greffer cette dichotomie sur la 
conception d'un univers dont les parties, de par leur constitution élémentaire, comportent des degrés différents de simplicité et de pureté matérielle.

Un glissement sémantique a permis d'articuler l'exposé cosmologique d'Aristote à l'exégèse biblique, car là où Aristote parle d'orbis comme étant le cinquième élément, les sources de Barthélemy ont écrit ether (DPR, VIII.5, 1. 17), restreignant de la sorte la portée du sens visé par Aristote. Néanmoins, l'absence de toute autre référence à la nature de l'espace des orbes planétaires ou à la composition des planètes limite notre compréhension des choix de Barthélemy à ce sujet. Si l'éther était l'élément propre aux cieux supralunaires, on pourrait difficilement expliquer la différence que Barthélemy institue entre le ciel cristallin et le firmament, les propriétés diverses attribuées aux planètes ou la définition de l'étoile en tant que " concentration de lumière " (stella est lux aggregata). Mais cette impasse n'est pas signalée par Barthélemy, ce qui est loin d'être l'exception à la règle ${ }^{132}$.

Les interactions thermiques que l'on imaginait entre les couches de l'univers sont abordés autant par binômes (cristallin - firmament, firmament - ciel des planètes, ciel des planètes - terre) qu'à l'échelle de l'ensemble de la machina mundi pour montrer qu'ils fonctionnent de manière à empêcher une combustion globale.

Le souhait de joindre exégèse biblique et théories cosmologiques entraîne des incohérences dans l'exposé des cieux invisibles également. Firmament, cristallin, empyrée, sont autant de couches célestes absentes des traités de Platon et d'Aristote, qui ont chacune leur place dans l'édifice de l'univers chrétien. L'ambiguïté sémantique du mot celum fait en sorte que le firmament est identifié au ciel conçu comme le cinquième élément, équivalence importée par le biais d'un passage du De causis et proprietatibus elementorum de Pseudo-Aristote ${ }^{133}$, alors que ce même statut est attribué, comme nous venons de le montrer, à l'éther par l'entremise des Météorologiques d'Aristote. Ailleurs, le firmament est qualifié de ciel à la suite immédiate d'une définition tirée du De celo et mundo d'Aristote qui exclut l'existence de plusieurs cieux ${ }^{134}$. Quelques aménagements ponctuels de la citation en cause pointent néanmoins vers l'existence du ciel empyrée :

DPR, VIII.2, 1. 104-106: Unde illic est vita fixa, scilicet extra ultimum, et est sempiterna que neque deficit neque finitur, et illa est vita vera ${ }^{135}$.

Alors que la définition d'Aristote affirmait qu'il n'existe ni lieu, ni corps, ni vide, ni plein, ni temps en dehors du ciel, et que la vie sempiternelle se déroule au sein de l'unique ciel, la glose intercalée par Arnold de Saxe, source de Barthélemy, fait référence à un espace situé au-delà de ce ciel où est la vraie vie. Ensuite, en remplaçant le comparatif melior par vera, Barthélemy met l'accent sur la différence séparant la condition existentielle de la vie terrestre de celle de la vie éternelle.

95 Au moyen de l'équivalence qu'il pose entre les deux termes, Barthélemy transfère toutes les propriétés physiques du celum aristotélicien au firmamentum chrétien, qui détient au plus haut degré une nature simple, uniforme, pure, incorruptible. De par son site, il est aussi le plus éloigné de la terre ${ }^{136}$, position qui selon la logique exégétique devait revenir au ciel empyrée. Quant à sa matière, l'identité posée par Pseudo-Aristote entre le ciel et le cinquième élément ${ }^{137}$ alloue au firmament une constitution distincte de celle des corps formés à partir des quatre éléments. Et lorsque Barthélemy, en reprenant l'opinion d'Augustin, le considère solidum et firmum, ce n'est pas tant pour souligner sa composition compacte et lourde que pour pointer vers sa fonction de séparateur des eaux célestes et des eaux inférieures, vers son caractère immuable et incorruptible. 

Barthélemy raille au passage ceux qui veulent prouver la nature froide de ces eaux par l'influence qu'elles exerceraient sur Saturne et qui soutiennent que la nature froide de cette planète située dans la proximité immédiate du firmament ne pourrait pas être expliquée autrement. Cet argument, recensé dans l'exégèse du passage biblique depuis Augustin, contrarie les ratione utentes et les philosophantes, qui mettent en cause le fait qu'il peut y avoir une relation de concorde entre deux éléments aussi contraires que l'eau et le feu, même s'il est écrit au livre de Job (XXV.2) que « Dieu fait régner la paix dans les hauteurs qu'il habite ». Cette objection fait écho aux arguments avancés par Guillaume de Conches dans sa Philosophia pour nier l'existence des eaux au-dessus du firmament. C'est à l'encontre d'un tel argument que Barthélemy introduit l'opinion des moderni, en l'occurrence Alexandre de Halès.

99 Sans privilégier l'état « gazeux » ou solide de l'eau (sous forme de vapeur ou de glace), la solution empruntée aux «modernes », qui ont pensé le problème autrement et avec davantage d'acuité ${ }^{141}$, met l'accent sur d'autres propriétés. En laissant de côté les qualités sublunaires de l'eau, notamment sa pesanteur et sa complexion froide et humide, Alexandre exploite ses caractéristiques formelles par un raisonnement analogique : la fluidité, mais surtout la transparence, qualité que le ciel aqueux partage tant avec le firmament qu'avec l'empyrée. Si les eaux au-dessus du firmament ne sont pas déterminantes pour les processus de génération et de corruption comme l'est l'eau du monde sublunaire, elles sont jugées nécessaires à la préservation de l'univers. En simplifiant l'exposé de la Summa 'Alexandri' qui faisait la distinction entre l'utilité et la nécessité du ciel cristallin ${ }^{142}$, Barthélemy laisse entendre que ce ciel est nécessaire, d'une part, à cause de sa nature transparente qui, tel le cristal, permet la diffusion de la lumière 
du ciel empyrée vers les étages inférieurs et, de l'autre, à cause de la mobilité partagée avec l'élément eau, mobilité qui déclenche et perpétue le mouvement des autres cieux.

Ces arguments concordent avec la hiérarchie des cieux que Barthélemy avait énoncée auparavant en prenant appui sur l'autorité d'Alexandre de Halès. Avec ce dernier, il identifiait l'empyrée à la première sphère de l'univers, immobile et source de lumière. Cette lumière se répandait sur le ciel cristallin pour traverser ensuite les autres cieux dont la capacité de diffusion diminuait au fur et à mesure qu'elle s'approchait de la terre.

\section{B. Complexion astrologique des planètes et des signes}

A partir du chapitre 8, Barthélemy focalise son exposé sur les réalités visibles propres au ciel firmament et aux sphères des planètes, ce qui n'est pas sans entraîner, une fois de plus, quelques incohérences. Le choix, d'une part, de séparer la présentation du Zodiaque et des constellations, qui pourrait laisser l'impression que les étoiles dites fixes ne sont pas localisées dans le même ciel que les signes zodiacaux, et, d'autre part, d'intercaler entre ces deux parties la série des planètes, est subordonné à une perspective astrologique des corps célestes qui ne tire des interprétations que de la nature des douze constellations zodiacales et des planètes. En puisant au legs de l'astrologie gréco-latine et arabe, l'exposé des signes (DPR, VIII.9-10) met en évidence les multiples regroupements possibles en fonction de leur complexion et de leurs rapports aux mouvements des planètes. Associés aux quatre éléments, les signes forment quatre réseaux d'affinités nommés triplicités : celle du feu (Bélier, Lion, Sagittaire), de la terre (Taureau, Vierge, Capricorne), de l'air (Gémeaux, Balance, Verseau), de l'eau (Cancer, Scorpion, Poissons). Chaque triplicité est rapportée à un des points cardinaux : l'ignée à l'Est, la terreuse au Sud, l'aérienne à l'Ouest et l'aqueuse au Nord. Le raisonnement analogique se poursuit et, selon la noblesse des planètes qui dominent chaque triplicité, rend les effets des signes orientaux plus forts que ceux des signes occidentaux, et les effets des septentrionaux plus forts que ceux des méridionaux. Néanmoins, la série des planètes regroupées sous les quatre triplicités au chapitre 9 diverge de celle qui est introduite au chapitre 10 pour décrire les propriétés de chaque signe zodiacal :

\begin{tabular}{|c|c|c|c|c|}
\hline $\begin{array}{l}\text { Triplicité } \\
\text { dominante }\end{array}$ & Planète & Jour & Nuit & Jour et nuit \\
\hline \multirow{2}{*}{ Orientale } & c. 9 & Sol & Luna & Saturnus \\
\hline & c. 10 & Sol & Iuppiter & Saturnus \\
\hline \multirow{2}{*}{ Occidentale } & c. 9 & Saturnus & Mercurius & Iuppiter \\
\hline & c. 10 & Saturnus & Mercurius & Iuppiter \\
\hline \multirow{2}{*}{ Septentrionale } & c. 9 & Venus & Luna & Mars \\
\hline & c. 10 & Venus & Mars & Luna \\
\hline \multirow{2}{*}{ Méridionale } & c. 9 & Saturnus & Mercurius & Iuppiter \\
\hline & c. 10 & Venus & Luna & Mars \\
\hline
\end{tabular}


La tradition astrologique grecque, latine et arabe illustrée par Ptolémée, Firmicus Maternus, Sahl b. Bisr et al-Qābisī concorde avec la série des triplicités mentionnée par Barthélemy au chapitre 10, ce qui laisse entendre que l'exposé du chapitre 9 est marqué par la corruption du texte-source.

103 Au sujet de l'exaltation des planètes, on constate d'autres incohérences, tant entre les informations disséminées à l'intérieur du livre VIII que par rapport à la tradition astrologique attestée entre autres par l'Introductorius d'al-Qābisī et par un tableau accompagnant les tables tolédanes dans nombre de manuscrits ${ }^{143}$.

\begin{tabular}{|c|c|c|c|}
\hline Signum & $\begin{array}{l}\text { Exaltationes secundum Alcab. et } \\
\text { Toletanas }\end{array}$ & DPR VIII.9 & DPR VIII.10 \\
\hline Aries & Sol in 190 & Sol in 180 & Sol in 190 \\
\hline Taurus & Luna in 30 & $\begin{array}{l}\text { Luna in } 30 \\
\text { Saturnus in } 210\end{array}$ & Luna in 40 \\
\hline Gemini & Caput Draconis in 30 & $\begin{array}{l}\text { Caput Draconis in } \\
30\end{array}$ & $\begin{array}{l}\text { Cauda Draconis in } \\
30\end{array}$ \\
\hline Cancer & Iupiter in 150 & & Iupiter in 150 \\
\hline \multicolumn{4}{|l|}{ Leo } \\
\hline Virgo & Mercurius in 150 & Mercurius in 150 & Mercurius in 150 \\
\hline Libra & Saturnus in 210 & & Saturnus in 210 \\
\hline \multicolumn{4}{|l|}{ Scorpio } \\
\hline Sagittarius & Cauda Draconis in 30 & $\begin{array}{l}\text { Cauda Draconis in } \\
30\end{array}$ & $\begin{array}{l}\text { Cauda Draconis in } \\
30\end{array}$ \\
\hline Capricornus & Mars in 280 & Mars in 270 & Mars in 280 \\
\hline \multicolumn{4}{|l|}{ Aquarius } \\
\hline Pisces & Venus in 270 & Venus in 280 & Venus in 270 \\
\hline
\end{tabular}

104 Si certaines parmi ces divergences (notamment les degrés des exaltations) peuvent être mises sur le compte de la corruption des manuscrits du DPR à une date assez haute, d'autres, comme celle que nous avons remarquée dans le cas des rapports entre planètes dominantes et triplicités, semblent remonter à des abrégés astrologiques utilisés pour la rédaction du chapitre 9 , dont les informations n'ont pas été confrontées aux sources employées pour le chapitre 10. L'identification erronée que Barthélemy établit entre les signes et les maisons entraine une confusion de plus, les propriétés de chacune des douze maisons étant conférées aux douze signes du zodiaque.

Quoi qu'il en soit de ces erreurs, il demeure que, par le biais de notions astrologiques comme les divisions ternaire ou quaternaire du zodiaque (respectivement en signes 
mutables, fixes et communs/cardinaux et en signes d'eau, de feu, de terre et d'air), les maisons (angulaires, succédentes et cadentes) et les aspects (trigone, carré et sextile), des notions que le corpus des Alchandreana par exemple n'utilisait pas encore ${ }^{144}$, Barthélemy se montre réceptif à un modèle épistémologique fondé sur l'influence que les signes et les planètes peuvent exercer sur le monde terrestre. Les relations géométriques qui fédèrent les constellations et les planètes en triplicités, faces, maisons, aspects confèrent un caractère objectif à leurs propriétés. Elles laissent apparaître que la disposition des astres n'est pas sans effet sur la Terre et que les diverses conjonctions ou positions planétaires peuvent entraîner des effets fastes ou néfastes dont il faut tenir compte, que ce soit dans la pratique médicale ou dans l'agriculture. Le savoir astrologique de Barthélemy l'Anglais se résume toutefois à ces considérations générales, sans faire d'allusion explicite à la question des nativités ou des interrogations.

\section{L'ordre des planètes et la durée de leur révolution}

Contrairement à la tradition antique ${ }^{145}$, l'espace planétaire décrit par Barthélemy est dédivinisé et dépourvu d'une nature éternelle. C'est dans le ciel empyrée qu'on trouve les créatures spirituelles, anges et béatifiés (DPR II.1-7 et VIII.6), alors que les démons peuplent l'air caligineux sous-lunaire où ils furent relégués après leur chute (DPR II.19-20). Bons ou mauvais, ces êtres ne revêtent pas le rôle d'intelligences motrices et n'influencent pas le cours des astres. Leur pouvoir est mis par Barthélemy en rapport avec l'axe Dieu-hommes et non pas avec le monde physique.

De la tradition astronomique latine, Barthélemy l'Anglais récupère l'image d'un univers à sphères concentriques plaçant au centre la terre, immobile et sphérique ${ }^{146}$. Des cercles intermédiaires constituent les orbites des sept planètes. L'extrémité est constituée, via une transposition théologique, par le ciel empyrée; lui aussi immobile, il fait ainsi pendant à la Terre.

Depuis l'Antiquité, astronomes et astrologues ont rangé les planètes selon différents critères. Les divergences entre les ordres proposés concernent surtout les planètes situées en-deçà de Mars, à savoir le Soleil, Vénus et Mercure. Selon Ptolémée, qui recourt à une série d'analogies, la succession des planètes serait la suivante: Saturne, Jupiter, Mars, Soleil, Vénus, Mercure, Lune, leur tempérament étant déterminé par la distance qui les éloigne de la chaleur du Soleil et de l'humidité exhalée par la Terre ${ }^{147}$. Le tempérament (surtout le degré d'humidité qui les caractérise) dicte le sexe attribué aux planètes, les quatre supérieures étant masculines, Vénus et la Lune féminines, alors que Mercure participe aux deux genres. Les planètes revêtent aussi ces attributs selon qu'elles précèdent le lever du Soleil (masculines) ou suivent son coucher (féminines) ${ }^{148}$. Le même ordre se retrouve dans la répartition planétaire des décans.

109 À l'encontre de cet ordre appelé chaldéen, Macrobe se montra partisan de la série platonicienne-égyptienne qui disposait les planètes de la façon suivante : Saturne, Jupiter, Mars, Vénus, Mercure, Soleil, Lune ${ }^{149}$. Sous l'influence de l'autorité de Pline, le système chaldéen constitua l'ordre planétaire largement adopté du IX ${ }^{e}$ au XII ${ }^{e}$ siècle ${ }^{150}$. Tous les diagrammes qui accompagnent les textes de Macrobe et Martianus Capella suivent cette disposition ${ }^{151}$. Mais le livre VIII du De nuptiis de Martianus Capella, qui plaçait Mercure et Vénus comme satellites du Soleil, rendait valides autant le modèle égyptien que le modèle chaldéen, puisque, selon que les deux planètes se situaient sur la partie supérieure ou 
inférieure de leur orbite, on pouvait définir différemment leur ordre par rapport au Soleil.

Résultat de l'utilisation de plusieurs sources, l'ordre des planètes dans l'exposé de Barthélemy l'Anglais varie d'un contexte à l'autre. La série des chapitres consacrés aux planètes (DPR, VIII.12-18) établit la succession suivante, qui correspond au modèle dit «égyptien»: Saturne, Jupiter, Mars, Vénus, Mercure, Soleil, Lune. La succession est confortée par quelques phrases des chapitres sur Mars et sur Mercure, où il est dit que Mars précède Vénus (DPR, VIII.14, 1. 7) et que Mercure se trouve entre l'orbite de Vénus et du Soleil ${ }^{152}$. Ce même ordre gouverne l'énumération des maisons des planètes ${ }^{153}$. Lui répond aussi le système qui associe la Lune et le Soleil à un seul signe zodiacal, les cinq autres planètes ayant chacune deux domiciles (qualifiés de premier et de secondaire dans le DPR). L'ensemble de ce dispositif est déjà soutenu par Macrobe, qui le reliait au thema mundi (la disposition des planètes lors de la création du monde), pour justifier la validité de l'ordre proposé par Platon ${ }^{154}$. C'est par rapport à cette disposition que les cinq planètes effectuent un mouvement rétrograde entre les deux maisons qui leur sont associées (Mercure de la Vierge aux Gémeaux et ensuite des Gémeaux à la Vierge, etc.).

111 Cependant, lorsqu'il est question de rappeler les influences de chaque planète sur le développement du fotus, l'ordre débute avec Saturne pour continuer avec Jupiter, Mars, le Soleil, Vénus, Mercure, la Lune et revenir à Saturne et Jupiter pour le huitième et le neuvième mois ${ }^{155}$. Se réclamant de l'autorité de Galien et de certains astrologues, cet ordre correspond au modèle chaldéen. On le retrouve chez Barthélemy dans le passage relatif aux décans également ${ }^{156}$. En revanche, dans la section réservée à l'exaltation des planètes ${ }^{157}$, l'ordre de présentation semble complètement aléatoire: Soleil, Vénus, Mercure, Lune, Saturne, Mars, Jupiter.

Dans le sillage de l'astronomie arabe, Barthélemy introduit deux autres corps célestes, la Tête et la Queue du Dragon (DPR, VIII.20), auxquels il n'attribue le statut de planète qu'en raison de leur nature et de leur effet; à la différence des autres planètes, ces " étoiles » suivent le mouvement du firmament passant du Lion au Cancer, du Cancer aux Gémeaux et ainsi de suite. Ce sont en réalité les nœuds lunaires qui gagnent de l'importance dans l'astrologie latine avec le recueil des Alchandreana composé vers le $\mathrm{X}^{\mathrm{e}}$ siècle ${ }^{158}$. Mais bien qu'il ne les tienne pas pour des planètes, Barthélemy leur impartit des signes d'exaltation et de chute et une durée de révolution. Il les décrit aussi comme des corps qui causent les éclipses de Soleil et de Lune : l'éclipse de Soleil se produit quand le Soleil et la Lune se trouvent en conjonction à moins de douze degrés de distance de la Tête ou de la Queue du Dragon ; l'éclipse de Lune a lieu lorsque la Lune côtoie la Tête ou la Queue du Dragon et que le Soleil est en opposition ${ }^{159}$.

113 Entre la partie dédiée aux planètes et celle qui traite des étoiles fixes, Barthélemy insère un chapitre consacré aux comètes qui semblent être parfois mobiles, parfois immobiles. Fondé sur la description traditionnelle de Bède le Vénérable, le chapitre ne fait pas état des nouvelles explications proposées par Grosseteste dans le De sphera et dans le De cometis $^{160}$. Barthélemy se limite à dire que les comètes ne traversent pas le Zodiaque comme le font les planètes, mais qu'elles se situent dans la voie lactée (galaxia). Une autre caractéristique, qui pouvait servir à l'exégèse biblique de l'épisode de l'étoile ayant guidé les mages lors de la naissance du Christ, est le cantonnement de l'apparition des comètes dans la partie septentrionale du firmament, alors que l'étoile des mages se déplaçait de l'est vers le sud-ouest. Cela rendait indéfendable l'objection qui tenait l'étoile des mages pour une comète. Sans avoir une identité astronomique claire, ni un mouvement régulier, 
les comètes se forment et apparaissent de façon aléatoire. Si le mouvement régulier des planètes et des étoiles fixes permet d'effectuer des calculs et des jugements astrologiques, le mouvement aléatoire des comètes leur attribue un statut d'exception, de signe, de prodige qui annonce des changements ${ }^{161}$.

\section{Interaction des sphères}

\section{Le mouvement}

114 Entre la terre et l'empyrée, les planètes effectuent deux mouvements, un mouvement diurne, entraîné par le firmament, d'Est en Ouest, et un mouvement inverse, dit naturel, d'Ouest en Est, contraire à la sphère des étoiles fixes ${ }^{162}$. Ces deux mouvements opposés sont censés empêcher le réchauffement de l'ensemble de l'univers et garantir de la sorte son équilibre énergétique, idée qui surgit à plusieurs endroits du De mundo et corporibus celestibus ${ }^{163}$.

Quelle est la force qui déclenche et maintient ces mouvements? Une citation du De proprietatibus elementorum de Pseudo-Aristote attribue le mouvement du firmament à un moteur d'une puissance infinie qu'une glose vient identifier à Dieu ${ }^{164}$. Plus loin dans le même chapitre, cette fois par l'entremise du De celo d'Aristote, il est dit que le mouvement du ciel est causé par la volonté d'un esprit (spiritus) ${ }^{165}$. Ce spiritus, qui joue selon Barthélemy le rôle de moteur, se rapproche-t-il des anges qui représentaient pour Averroès les moteurs célestes ${ }^{166}$ ? Impossible de répondre, en raison des découpages réalisés par Barthélemy et ses intermédiaires. On peut seulement dire que l'idée d'un principe moteur extrinsèque domine, ce qui évacue la théorie de l'anima mundi présente au XII ${ }^{\mathrm{e}}$ siècle, et attribue au ciel un mouvement circulaire et uniforme.

116 Dans cette mécanique des deux mouvements contraires, le ciel des planètes a pour fonction de contrer le mouvement inverse du firmament. Alors que le mouvement du firmament est tenu pour être le plus rapide de tous ${ }^{167}$, la vitesse de rotation de chaque planète n'est pas clairement déterminée par Barthélemy. De façon générale, les étoiles sont définies comme étant de nature très rapide ${ }^{168}$, les deux pôles y compris ${ }^{169}$. La longueur de l'orbite que décrivent les planètes fait en sorte que certaines parmi elles paraissent accomplir leur révolution plus vite que d'autres ${ }^{170}$. Si la distance qui nous sépare du corps céleste influence l'évaluation de sa vitesse de déplacement, un autre élément peut fausser l'estimation: la splendeur du corps. C'est le cas du Soleil qui se déplace "plus vite qu'une flèche", mais dont l'intensité lumineuse nous empêche d'évaluer la vitesse réelle ${ }^{171}$. Un passage associé à l'autorité de Macrobe sur les mouvements de Vénus et de Mercure laisse entendre qu'elles ont des vitesses différentes en fonction du positionnement de l'une par rapport à l'autre ${ }^{172}$. Les deux adjectifs censés exprimer cette variation de la vitesse (segnior, celerior) sont associés au verbe être, alors qu'il est plus logique de comprendre que la vitesse de Vénus semble être plus lente ou plus rapide en fonction de sa position par rapport à Mercure. Quoiqu'il en soit, la vitesse est présentée comme un élément caractéristique des corps célestes. Par ailleurs, elle se trouve parmi les facteurs à l'origine des effets de la Lune sur le corps humain, tout comme le sont la proximité de la Lune par rapport à la Terre et sa vertu occulte ${ }^{173}$.

117 Du point de vue de l'emboîtement et du mouvement des planètes, le chapitre De duplici motu planetarum montre que Barthélemy connaît la théorie des épicycles et des excentriques $^{174}$ - qu'il résume maladroitement (DPR, VIII.11, 1. 118-171). Chaque planète 
est dotée d'un mouvement qui lui est propre et qu'elle effectuerait sur son orbite dont le centre n'est pas la terre. Barthélemy identifie ce cercle avec l'excentrique, alors qu'il s'agit de l'épicycle. Un autre mouvement porte la planète sous le Zodiaque, dont elle parcourt les signes dans des périodes toujours variables. La définition, elle aussi erronée, de l'excentrique en tant que « cercle qui traverse le centre de la terre tout en ayant son propre centre au-delà du centre de la terre » est complétée par une typologie qui confond plusieurs notions astronomiques ${ }^{175}$. D'une part, l'excentrique est distinguée en cercle déférent et cercle équant, d'une autre, Barthélemy assigne aux planètes, "selon Ptolémée ", trois cercles : l'équant, le déférent et l'épicycle. À partir de la définition du déférent, il n'est pas clair si Barthélemy traite du déférent géocentré ou de l'excentrique. L'équant, quant à lui, classé incorrectement par Barthélemy parmi les cercles plutôt que comme un point, relève d'un modèle dans lequel le déférent excentrique a un autre centre que la Terre et l'équant est un point distant du centre de la terre par rapport auquel Ptolémée avait défini le mouvement uniforme de l'épicycle des planètes ${ }^{176}$. Grâce à ces modèles, Ptolémée avait remplacé le système des sphères concentriques tout en préservant le géocentrisme et l'idée de mouvement circulaire uniforme des planètes. Barthélemy l'Anglais, qui adopte le système des sphères concentriques également, ne saisit pourtant pas la divergence qui le sépare du modèle des excentriques et des épicycles. Il ne souligne pas non plus l'importance de ces modèles pour comprendre par exemple l'inégalité des saisons.

118 Pour expliquer le mouvement apparent des planètes, le modèle des épicycles situait sur la partie supérieure de l'épicycle le mouvement direct de la planète, la portant d'Ouest en Est, mouvement qui devenait retrograde lorsqu'elle pénétrait à l'intérieur du déférent en parcourant la partie inférieure de l'épicycle. Lorsque la planète passait d'un mouvement à l'autre, elle semblait s'arrêter, moment qui fut nommé station. Les définitions que reprend Barthélemy rapportent ces trois étapes du mouvement de la planète au début et à la fin de l'espace du signe zodiacal et non pas à la circonférence de l'épicycle comme le fait par exemple l'auteur de la Sphera cité par Vincent de Beauvais ${ }^{177}$. À la différence de ce dernier, qui laisse entendre qu'à l'exception du Soleil toutes les planètes connaissent la station et le mouvement rétrograde, Barthélemy attribue cette exception autant au Soleil qu'à la Lune. Bien plus, selon une théorie qu'il associe au nom d'Alfraganus, les mouvements rétrogrades et stationnaires sont le résultat de la force de répulsion et d'attraction exercée par les rayons du Soleil. Barthélemy s'empresse de noter que Ptolémée détermine d'autres causes pour ces phénomènes, mais il n'en donne pas les détails ${ }^{178}$.

Barthélemy aborde à deux reprises la durée de révolution des planètes ${ }^{179}$, la première fois dans un résumé intégré à l'aperçu astrologique du chapitre $11^{180}$, ensuite dans les chapitres consacrés à chaque planète en particulier (DPR, VIII.12-18). Dans l'aperçu astrologique, Vénus est placée entre Mercure et la Lune (conformément à l'ordre proposé dans le Timée et, plus tard, dans les versions du Liber Alchandrei), alors que, dans l'exposé réservé à chaque planète, Barthélemy, sous l'autorité de Ptolémée ${ }^{181}$, range Vénus entre Mercure et Mars, ce qui correspond à la durée respective de leurs révolutions, 348 jours pour Vénus et 338 pour Mercure. Les informations concernant la station de Mars dans chaque signe divergent elles aussi d'un endroit à l'autre: dans l'aperçu, Barthélemy indique 45 jours (durée que l'on trouve entre autres dans le Liber Alchandrei), ce qui implique en fait un cycle de 540 jours et non de deux ans comme l'indique la suite du texte. Au chapitre $14^{182}$, il parle de 60 jours et 21 heures (selon le texte de Pseudo- 
Ptolémée, connu via Arnold de Saxe), ce qui équivaut à un cycle de deux ans. Même état de choses pour la Lune, dont la station est fixée dans le chapitre 11 (1. 22-25) à « 2 jours et demi et 6 heures et deux tiers d'heure » et à un cycle de 32 jours et 8 heures, alors qu'au chapitre $18^{183}$ elle est de " 2 jours et 6 heures et deux tiers d'heure » pour un cycle de 28 jours. Les deux valeurs données pour la durée du cycle sont erronées, car on aurait dû avoir dans le premier cas un cycle de 33 jours et 8 heures, dans le deuxième un cycle de 27 jours et 8 heures, qui se rapproche de la valeur acceptée de nos jours pour la révolution sidérale de la Lune (27 jours $7 \mathrm{~h} 43 \mathrm{mn} 11,5 \mathrm{~s})^{184}$.

Ces incohérences font supposer que, pour l'aperçu du mouvement des planètes inséré au chapitre 11, Barthélemy a puisé dans un texte différent (qui pourrait être proche du Liber Alchandrei) de celui qu'il a employé pour la partie consacrée à chaque planète en particulier (dans ce cas Arnold de Saxe ou un texte apparenté à ce dernier pourrait en être la source). Par ailleurs, les incohérences marquaient déjà les sources utilisées par Barthélemy : le traité de Pseudo-Ptolémée spécifie par exemple les domiciles des planètes selon l'ordre platonicien (nommé « égyptien » par Macrobe), alors que la durée des cycles de chaque planète rapproche le texte de l'ordre chaldéen. D'autres chiffres sont donnés par l'entremise du De proprietatibus elementorum de Pseudo-Aristote ${ }^{185}$; celui-ci, en adoptant la série Lune, Mercure, Vénus, Soleil, Mars, Jupiter, Saturne, indique pour Mercure et Vénus une durée identique de 10 mois.

La révolution des étoiles et des planètes est à l'origine des principales catégories temporelles (l'an, le mois, le jour). Barthélemy rappelle cet effet entraîné par la périodicité des mouvements célestes. Mais, en juxtaposant, via Arnold de Saxe, une citation tirée du De proprietatibus elementorum de Pseudo-Aristote et une autre de Macrobe ${ }^{186}$, il confond la précession des équinoxes (cycle d'environ 26000 ans dans lequel les étoiles fixes se déplacent imperceptiblement pour revenir à la même position, que Ptolémée avait évalué à 36000 ans) avec l'annus magnus (intervalle dans lequel les planètes reviennent aux positions qu'elles occupaient à la formation de l'univers, ou bien intervalle dans lequel les planètes s'alignent parfaitement par rapport à un point de la sphère des étoiles fixes) ${ }^{187}$. Barthélemy, qui reprend des passages déjà décontextualisés et assemblés par Arnold de Saxe, se soucie moins de concilier les deux valeurs données par ses sources (36 000 selon Pseudo-Aristote, 15000 selon Macrobe) que de mettre en garde contre toute tentative de conjecturer la fin du monde à partir des mouvements des astres 188 .

Les relations entre les planètes se traduisent aussi en rapports harmoniques que Platon avait déjà définis dans le Timée ${ }^{189}$. De toute la terminologie musicale présente dans l'œuvre de Martianus Capella, Barthélemy l'Anglais ne conserve la trace que des termes communs stridor, harmonia, concentus; seul le rapport harmonique entre Vénus et le Soleil recouvre une notion plus technique: tribus semitoniis. Cette donnée, connue via le commentaire du De nuptiis de Rémi d'Auxerre, remonte à Pline et reflète une disposition chaldéenne des planètes. Mais il n'est pas certain que les compilateurs aient eu pleinement conscience de la position héliocentrique de Mercure et de Vénus défendue par Martianus Capella. C'était sur la base de ces rapports numériques définis entre le Soleil et la Lune, d'une part, et toutes les autres planètes, de l'autre, que Ptolémée, Macrobe et d'autres ont démontré le caractère bénéfique de Jupiter et de Vénus et la nature maléfique de Saturne et de Mars. S'il est difficile de se prononcer sur l'intérêt de Barthélemy pour les rapports harmoniques des planètes dans la mesure où sa connaissance des œuvres de Martianus Capella et de Macrobe est de seconde main, il reste 
qu'il répète à plusieurs reprises, autant dans le livre VIII que dans le livre XIX, l'idée de l'existence d'une musique des sphères.

Le mouvement des cieux, la position des planètes et leurs conjonctions, les phases de la Lune sont tous censés produire des effets sur la Terre. Ainsi, le firmament est la cause effective du processus de génération et de corruption par la vertu de la lumière qu'il renvoie vers le centre de l'univers, la Terre. C'est pour limiter la puissance de ses rayons qu'ont été intercalées les sphères des planètes, qui, différentes par leurs dimensions, font varier la chaleur et le froid à la surface de la Terre et assurent donc une sorte d'équilibre thermique au sein du mundus. Le mouvement du firmament se répercute aussi dans d'autres phénomènes, comme les marées ${ }^{190}$.

L'observation des phases de la Lune et son importance pour la pratique médicale est un lieu commun depuis l'Antiquité. En reprenant des sources astrologiques et médicales, Barthélemy glisse dans les chapitres sur la Lune quelques considérations sur les influences que tout médecin se doit de prendre en compte. Un extrait tiré du De iudiciis infirmitatis attribué à Hippocrate, plus couramment connu sous le titre Astronomia ou Astrologia Hippocratis ${ }^{191}$, vient rappeler brièvement les effets de la Lune ${ }^{192}$. Jouxtées à d'autres passages empruntés aux Pronostics d'Hippocrate et au De creticis diebus de Galien, ces considérations dressent un petit tableau des principales thèses d'astrologie médicale qui circulaient au début du XIII siècle. En revanche, il est plus difficile d'évaluer la portée pratique que leur prêtait Barthélemy.

Le lever et le coucher des constellations sont également des phénomènes sur lesquels reposent les prédictions météorologiques, que ce soit au sujet des vents, des tempêtes ou des pluies. Barthélemy inventorie ainsi par l'entremise de Bède et d'Albumasar différents éléments prédictifs, rapportés au Soleil et à la Lune ou encore à la position des constellations. Les comètes entrent aussi dans cette catégorie des phénomènes annonciateurs de changements climatiques et même de bouleversements politiques ${ }^{193}$. Comme le rappelle Barthélemy, agriculteurs ${ }^{194}$, navigateurs ${ }^{195}$, médecins ${ }^{196}$ pouvaient tous tirer profit de ce type d'observations astronomiques.

De l'Empyrée à la Lune, les sphères et les astres interagissent les uns avec les autres et exercent leur influence sur la Terre. Barthélemy se montre en somme réceptif aux différents types d'interactions à l'aide desquelles il construit l'image d'un univers dont les strates ne sont pas séparées. Régularité et périodicité des mouvements célestes, trajet aléatoire des comètes, influence géocentrée, sont les caractéristiques qui se dégagent de la compilation de Barthélemy, malgré la nature erronée ou lacunaire des informations. Dotées de vertus dont la force change en fonction des déplacements et des conjonctions des astres les uns par rapport aux autres, les réalités célestes sont intégrées à un système aux significations multiples. Si Barthélemy ne qualifie plus d'infernum ce monde ici-bas comme le faisaient au XII ${ }^{\mathrm{e}}$ siècle Bernard Silvestre et Hugues de Saint-Victor, il continue cependant à décrire la terre comme un corps sis au niveau le plus bas du cosmos et dépourvu de noblesse, qui, par rapport à la grandeur et à la beauté du ciel, ne laisse apparaitre que faiblement la force créatrice de Dieu (DPR XIV.1). En lui attribuant ce statut, Barthélemy perpétue le thème du contemptus mundi qui est explicitement exprimé dans le premier chapitre du livre VIII ${ }^{197}$. 


\section{La lumière} prend le relais : lorsqu'elle traverse l'air ou un corps transparent, cela suppose que deux corps se retrouvent au même endroit ${ }^{203}$. Quelques exemples viennent démontrer la possibilité de la coexistence de deux corps dans un même espace sans que leur corporéité, leur forme substantielle, disparaisse: le mélange d'eau et de cendres, la fusion de plusieurs faisceaux lumineux, la présence au même endroit d'un corps glorifié et d'un autre non-glorifié. Au sujet de ce dernier exemple, il convient de signaler que Barthélemy, en soutenant que deux corps glorifiés ne peuvent pas se trouver au même endroit, contredit Richard Fishacre ${ }^{204}$ dont les arguments formulés dans la questio de luce apparaissent pourtant à plusieurs endroits presque à la lettre dans le texte du DPR. On retrouve une partie des exemples et des arguments résumés par Barthélemy dans le commentaire des Sentences rédigé par Bonaventure dans les années $1250^{205}$. En somme, le développement de la question de la lumière compilé par Barthélemy se ressent des thèmes qui alimentent à la même époque l'exposé de Fishacre et le commentaire de Bonaventure.

131 La lumière-lux part du ciel empyrée et se diffuse jusqu'au centre du monde ${ }^{206}$. Pour gloser le texte de la Genèse, Barthélemy reprend la doctrine de Basile de Césarée et identifie cette lumière à la lumière primordiale du Fiat lux qui a été ensuite conférée aux astres du ciel formés au quatrième jour. Depuis lors, ces astres transmettent la lumière primordiale. Par sa diffusion à l'échelle de l'univers, elle participe à la formation de tout elementatum, corps composé d'éléments contraires ${ }^{207}$. Comme il a déjà été dit dans le chapitre consacré 
au firmament ${ }^{208}$, c'est la lumière du firmament qui, par la concentration des rayons favorisée par la forme sphérique du monde, participe à la génération de toutes les res. On pourrait conclure que la matière de la lumière est constituée par les particules primordiales créées par Dieu et que la forme lui est donnée par le ciel empyrée, alors que la lumière des autres cieux et du Soleil est synonyme plutôt de lumen - luminosité - que de lux. Une telle interprétation semble appuyée aussi par la définition de l'action du firmament qui sui luminis virtutem [...] ad terram tamquam ad proprium centrum dirigit (DPR, VIII.2, 1.160-162), du Soleil en tant que fons totius luminis, cuius irradiatione superiora et inferiora illustrantur (VIII.17, 1.3-4), de la lux qui continet omnia corpora, respectu quorum penitus formalis est, licet in se sit materialis et situalis (VIII.29, 1.132-133). Comme on le souligne plus bas, chez Grosseteste, le lumen est une substance quasi-spirituelle émise de même par les sphères célestes, et "incorporée " dans les corps physiques, où elle est source de divers phénomènes comme la couleur, la chaleur, ou un autre type de perception par les sens ${ }^{209}$.

La réécriture d'un passage tiré du Fons vite transfère à la lumière la vertu qu'Avicebron (Ibn Gabirol) associait à l'âme ${ }^{210}$. De la sorte, la virtus lucis prend la place de l'actio anime sensibilis, mais opère le même effet dans le corps des animaux, à savoir la sensation et le mouvement. L'action de la lumière est nécessaire à tel point que sans elle tout corps animé serait anéanti ${ }^{211}$. Substituer la lumière à l'âme n'est peut-être pas le résultat d'un glissement sémantique aléatoire, puisque plusieurs théologiens de la première moitié du XIII ${ }^{e}$ siècle ont discuté des ressemblances entre la nature de la lumière et celle de l'âme ${ }^{212}$. Avec ses multiples propriétés, la lumière, telle que la décrit Barthélemy, revêt le statut de cause universelle, ce qui n'est pas sans rappeler l'anima mundi que les théologiens du XII siècle ont évoquée pour rendre compte de la cohésion de l'univers.

Dans le chapitre 30, De splendore, Barthélemy se limite, à partir d'Augustin et de Basile, à dire que la "splendeur » provient comme un flux de la substance de la lumière, sans mélange avec une autre nature, et lui est coéternelle en durée, à l'image de la luminosité qui coexiste au feu ${ }^{213}$. C'est le sens qui est donné à la splendeur irradiée par le ciel empyrée, qui diffuse splendeur et lumière vers le ciel cristallin ${ }^{214}$. Mais cette splendeur est-elle de même nature tout au long de la procession de la lumière vers la Terre ? Est-elle identique à l'éther, qui selon l'étymologie reprise d'Isidore, signifie splendeur ${ }^{215}$ ? Ou encore à celle du Soleil qui illumine le ciel et tout ce qui se trouve en-dessous ${ }^{216}$ ? La distinction entre lux et lumen suppose de distinguer aussi entre splendor luminis et splendor lucis, et l'on soupçonne l'embarras devant cette nuance lorsque l'éther est mis en rapport avec perpetuus splendor luminis sive lucis (DPR, VIII.5, 1. 4-5). La diversité des sources citées vient compliquer elle aussi toute tentative de tirer des conclusions nettes au sujet de cette terminologie.

\section{Le diaphane}

Ce qui conditionne le passage de la lumière du ciel empyrée vers les cieux inférieurs est leur nature transparente, nature que vient souvent qualifier la famille du mot diaphanus ${ }^{217}$ . Utilisés à propos du ciel (DPR, VIII.2, trois fois) et de la lumière (DPR, VIII.29, une fois ; DPR, VIII.31, deux fois; DPR, VIII.32, une fois), ces termes sont adjoints à des synonymes explicatifs qui trahissent la nouveauté du mot: claritas, transparentia - natura transparens, perspicuitas - perspicuus. Au sujet de la lumière, le binôme diaphanus - transparens répond à l'emploi conjoint de deux adjectifs diaphanus - perspicuus par Barthélemy au livre XIX, cette fois à propos de la couleur. 
En ce qui concerne le livre VIII, l'adjectif diaphanus apparaît dès son début, au chapitre 2 d'abord, où, après avoir énuméré les sept ciels, de l'aérien à celui des anges (l'empyrée), Barthélemy décrit l'aérien comme un ciel «interstitiel », car il n'est affecté ni par les lourdes vapeurs d'eau, ni par celles de la terre. C'est un ciel plus imperméable aux qualités du monde inférieur, appelé «ciel » en vertu de sa pureté et de son caractère "diaphane », c'est-à-dire de sa "nature transparente ». Quelques lignes plus loin, à la suite de la citation attribuée au De sensu et sensato, d'après une fausse référence du Liber celi et mundi, Barthélemy utilise à nouveau l'adjectif. Le ciel, dit-il, est d'une nature resplendissante, mais différente d'une à l'autre de ses parties. C'est en effet dans les étoiles que se trouve la plus grande concentration de lumière. Suit un passage où sont énumérées différentes propriétés du ciel compris dans son ensemble, parmi lesquelles sa nature diaphane (dyaphanitas). Deux termes placés à sa suite, sive transparentiam et perspicuitatem, sont censés compléter l'image, sans que l'on puisse dire si la synonymie annoncée par sive se reporte aux deux termes ou à la transparentia uniquement ${ }^{218}$. Dans cet extrait, Barthélemy recourt aussi au terme classique pour expliquer le caractère transparent, la claritas.

Dans le même chapitre ${ }^{219}$, Barthélemy cite à nouveau le Liber celi et mundi d'Aristote à travers Arnold de Saxe, puis un autre passage au vocabulaire redondant à propos de la propriété diaphane du ciel, où l'emploi des catégories aristotéliciennes prédomine comme c'était déjà le cas dans les extraits examinés plus haut. Bien que nous n'ayons pas pu identifier l'origine de ces deux passages cités dans le sillage du Liber celi et mundi, il est probable qu'ils soient issus du même ensemble ou du même enseignement sur la nature du ciel que les phrases tirées du commentaire à l'Hexaëmeron de Grosseteste.

L'adjectif diaphanus refait surface dans le chapitre sur la lumière et ses propriétés ${ }^{220}$, au sein d'une discussion dont la thématique et l'argumentation sont proches de la Questio de luce de Richard Fishacre, qui, lui, n'emploie pourtant pas ce terme.

L'utilisation des mots "diaphane » et "transparence " témoigne d'un changement de terminologie, accompagnant d'une part la vivacité des discussions sur les facultés de l'âme, et d'autre part les progrès de l'optique, la question de la vue et du regard reliant les deux thématiques. En effet, le concept de diaphane est lié, au XIII ${ }^{e}$ siècle, à l'exposé des facultés de l'âme et en particulier la vue, dans le livre II et le début du livre III du De anima

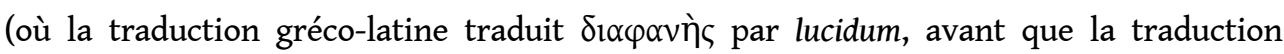
arabo-latine diffusée avec les traductions du commentaire d'Averroès donne diaphanum), et à la théorie aristotélicienne de la vision telle qu'on la trouve exposée dans le De sensu (III, 439b11-12).

Comme l'adjectif diaphanus, le terme transparentia - transparens est d'un usage récent puisqu'il n'apparaît que dans la seconde moitié du XII siècle. A. Vasiliu note que Dominique Gundissalvi utilisait encore l'adjectif classique translucens pour traduire le grec

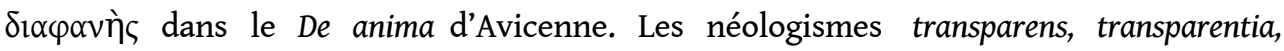
transpareo, associés à la lumière, à la vue et aux matières transparentes comme l'air, l'eau ou le verre, ont été probablement introduits en latin par Burgundio de Pise qui en fait usage dans la traduction du De generatione et corruptione d'Aristote et du De natura hominis de Nemesius d'Emèse ${ }^{221}$. Nos propres investigations confirment que l'usage des familles des termes diaphanus et transparens est précoce chez Barthélemy l'Anglais ${ }^{222}$. La plus ancienne occurrence de diaphanus dont nous avons trouvé trace apparaît dans la traduction du traité d'optique d'Ibn al-Haytham (Alhacen) entreprise vers 1200 sous le titre De aspectibus ${ }^{223}$. Le terme se diffuse ensuite de plus en plus. Au début du XIII ${ }^{e}$ siècle, la 
traduction des commentaires d'Averroès ${ }^{224}$ en atteste et les traités de Robert Grosseteste figurent parmi les plus anciens textes témoignant de son utilisation: le De iride, le De cometis comptent parmi ces témoins, mais Grosseteste préfère en général le traditionnel perspicuus.

Le calque latin du terme grec était-il le résultat d'un besoin terminologique entraîné par les débats en matière d'optique? Comportait-il pour ceux qui l'ont préféré aux plus traditionnels pervius, perspicuus, translucens un degré de précision supplémentaire ? Il est intéressant de constater que d'une traduction à l'autre du De anima d'Aristote, par exemple, le terme grec a fini par s'imposer. Ainsi la définition du transparent (lucidum) donnée par Aristote est traduite par Jacques de Venise dans les termes suivants :

Lucidum autem dico quod est quidem visibile, non autem secundum se visibile ut simpliciter est dicere, sed propter extraneum colorem. Huiusmodi autem est aer et aqua et multa planorum. Non enim secundum quod aqua est neque secundum quod est aer, lucidum est, sed quoniam est natura eadem in his utrisque et in perpetuo superius corpore. Lumen autem est huius actus lucidi secundum quod est lucidum ( De anima, II.7, 418b).

141 Cette définition connaît quelques ajouts intéressants dans le Speculum naturale de Vincent de Beauvais et le De proprietatibus rerum de Barthélemy l'Anglais :

Lucidum autem sive diaphanum dico [...]. Huiusmodi sunt aer et aqua multaque planorum id est perspicuorum. [...] Lumen autem sive lux est actus lucidi sive diaphani secundum quod est lucidum (SN, 25.39).

Lux est actus et perfectio lucidi seu perspicui ( DPR, XIX proem., éd. Frankfurt : 1134).

Chez les deux compilateurs, des synonymes terminologiques sont introduits pour le mot lucidum, ce qui rappelle les distinctions qu'Albert le Grand abordait dans son De homine entre transparens et perspicuus ${ }^{225}$. La révision de la traduction de Jacques de Venise faite par Guillaume de Moerbeke avant 1270 a eu pour effet que diaphanum supplante lucidum sans que l'on puisse déterminer si ce choix fut dicté par une pensée plus raffinée des phénomènes optiques ou simplement par une préférence donnée aux hellénismes par Guillaume de Moerbeke.

143 Exposée dans le cadre de la théorie du passage de la puissance à l'acte de la vue, la notion de diaphane est corrélée par Aristote à la lumière, à la vue, au visible, à la couleur et correspond à un «milieu ", à un espace interstitiel où l'organe de la vue peut recevoir l'image des corps qui s'actualise. Dans le De anima, le diaphane est donc présenté comme la manifestation de la lumière en tant qu'acte pur. Comme le dit A. Vasiliu, « le diaphane... désigne une rencontre particulière de la lumière et des corps et un certain mouvement ... qui accompagne l'action de la lumière, c'est-à-dire son énergie illuminative ${ }^{226}$. En tant qu'intervalle, le diaphane serait une entité non-corporelle, et «consisterait [...] dans l'installation au sein d'un milieu quelconque, comme l'air ou l'eau, d'une structure hétérogène, d'une disposition commune à tout milieu de devenir milieu-réceptacle-dequelque-chose, comme la vue et comme la visibilité de tout objet, sous l'action d'un agent séparé [...] mais en fait invisible en soi, comme la lumière $»^{227}$. Le diaphane serait donc une « nature propre à tous les corps et en même temps 'matière' immatérielle à même de recevoir en elle la lumière $»^{228}$. En ce sens, le diaphane est une qualité qui donne à tout corps la capacité de retenir et de transmettre la lumière.

L'exposé sur la couleur dans lequel Barthélemy l'Anglais traite de la notion de diaphane pourrait s'être inspiré des commentaires du De anima comme du De sensu, mais le terme lui-même apparaît ou non selon la traduction médiévale utilisée. L'adjectif se trouve 
appliqué à la définition de la couleur dans la version du De sensu qui est accompagnée du compendium d'Averroès traduit par Michel Scot, alors que la translatio vetus du De sensu utilisait encore perspicuus ${ }^{22}$. Barthélemy ne se réfère cependant pas au De sensu dans les chapitres du DPR qu'il consacre aux facultés des sens (DPR, III.17-21) et aux organes sensoriels (DPR, V.5-8, V.12-13, V.21-22). En revanche, il cite nommément ce traité au livre VIII.2, De celorum distinctione: Dicit enim Aristoteles in libro De sensu et sensato : Celum ex sui natura splendidum est, sed in partibus eius est differentia. Nam in stellis est maior aggregatio luminis quam in aliis partibus ipsius celi. Comme l'a montré G. Galle ${ }^{230}$, cette référence suivie d'une occurrence du mot dyaphanitas - ne vient pourtant pas du De sensu, mais du Liber celi et mundi de Pseudo-Avicenne (c. 14), qui renvoie par erreur au De sensu. Il est donc a priori étonnant de trouver ailleurs dans le DPR des citations, réelles cette fois, du De sensu : au livre XIX, dans le chapitre sur la couleur, ainsi que dans le chapitre sur le goût ${ }^{231}$.

Plutôt qu'un indice du recours direct à la traduction du De anima ou du De sensu et sensato d'Aristote, l'utilisation du terme diaphanus chez Barthélemy pourrait être le témoignage de l'influence de Robert Grosseteste sur les débats contemporains. Le De colore de Grosseteste aurait été écrit vers 1225 ou entre 1230 et $1233^{232}$, ce qui signifierait que le $D P R$ a connu une rédaction lente, à partir d'une documentation très diverse, répartie selon les livres, puisque c'est seulement au début du livre XIX que Barthélemy copie

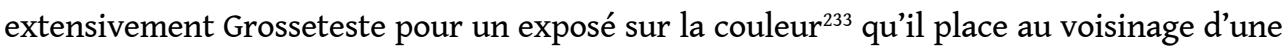
citation tirée du De sensu et sensato dans la translatio vetus ${ }^{234}$ et d'un passage sur le même sujet fondé sur le De anima d'Aristote :

Rei enim corporalis visibilis superficies et terminus colorum differentias, quas recipit ex natura mixtionis elementorum, et dominio in corpore composito dicit, quia mixtio perspicuo et diaphano per actualem unionem lucis radiantis super ipsam terminabilem et perspicui corporis superficiem visui representat. Et propter hoc dicit Aristoteles in secundo libro de anima, quod color est motivus vel immutativus visus secundum actum lucidi, nam lux est actus et perfectio lucidi seu perspicui. Naturam enim colorum in corpore mixto ex alicuius elementi dominio essentialiter existentis producit lux ad actum immutationis visus (DPR XIX, prooemium, éd. Frankfurt : 1133).

146 En ce qui concerne le livre VIII, l'exposé de Barthélemy emprunte beaucoup au commentaire à l'Hexaëmeron, transmis, comme nous l'avons dit ci-dessus, à travers la compilation de l'Experimentator. Par exemple, la définition de la couleur en tant que "lumière incorporée ", lux incorporata in superficie humidi perspicui (DPR, VIII.29, 1. 185) est proche de celle donnée par Grosseteste qui l'attribue comme Barthélemy à Augustin. Ludwig Baur a déjà fait le lien entre les doctrines professées par Grosseteste sur la lumière " donatrice de forme » et les chapitres 29 (De luce et eius proprietatibus, 1. 114-139, en particulier 1. $123 \mathrm{sq}$. pour les échos du commentaire à l'Hexaemeron où il est aussi question de la lux formalis) et 31 (De lumine) du livre VIII de Barthélemy ${ }^{235}$.

A l'époque de Barthélemy, la notion de « diaphane » est incontestablement répandue dans les milieux anglais, et en particulier franciscains. Ainsi, le mot diaffonum se trouve dans l' abbreviatio d'Oxford des traités aristotéliciens, toujours dans la partie concernant le $D e$ sensu, et en lien avec le concept de lux incorporata cher à Grosseteste. On y retrouve la question posée chez Barthélemy et Richard Fishacre, de la coprésence en un même lieu de deux corps, dont l'un est la lumière ; cette rencontre entre lumière et un corps diaphane est précisément ici l'explication du phénomène de la couleur tel qu'il est défini dans le $D e$ $s^{s e n s}{ }^{236}$. De telles abbreviationes du corpus vetustius (ou corpus de summulae) sont contenues 
dans un certain nombre de manuscrits qui illustrent le développement de l'aristotélisme dans la première moitié du XIII siècle à la Faculté des arts d'Oxford ${ }^{237}$.

Du livre VIII au livre XIX, le diaphane a-t-il la même fonction théorique, recouvre-t-il les mêmes propriétés? Barthélemy ne donne pas de détails à ce sujet. On peut déduire des différents contextes où le terme apparaît que, dans l'espace céleste, la transparence est une propriété des cieux nécessaire à la transmission de la lumière sans qu'aucun obstacle ne s'interpose. Les traités d'Aristote ne s'intéressent pas à ce diaphane, mais à celui qui participe au processus de la vision et à la perception de la couleur dans le monde sublunaire. Cette dernière acception du terme est évoquée dans les livres III.17 et XIX du DPR. Il demeure néanmoins que le terme diaphanus ne s'impose pas dans tous les contextes où il est question de transparence, et que Barthélemy, à travers les traductions et les commentaires qu'il compile, continue à utiliser perspicuus et autres synonymes à ses côtés.

Qu'il s'agisse des hiérarchies célestes, de la complexion des constellations, des interactions entre les sphères, de leur mouvement, de leur ordre, de la durée des révolutions célestes, l'examen approfondi du traitement de toutes ces questions par Barthélemy conduit à un même constat : le livre sur le cosmos et les corps célestes est une œuvre de compilation de notions astronomiques et astrologiques élémentaires, souvent incomplètes ou insuffisamment définies, qui n'évite ni les répétitions, ni les contradictions, ni les à-peu-près, et dont les références aux sources visent à créer un effet d'érudition en masquant les véritables véhicules de la documentation. Au-delà de ce bilan peu flatteur, les intérêts du livre apparaissent. Son objectif n'étant pas d'ordre scientifique, dans sa préoccupation à collecter le maximum de propriétés caractéristiques de tel élément du cosmos, sa finalité doit être trouvée ailleurs : dans le rôle de support à l'interprétation allégorique que jouent les excerpta d'autorités; c'est là que les notes marginales dévoilent toute leur importance. Accompagné de cet apparat théologicomoral, le livre VIII témoigne d'un monde de correspondances fondées sur les proprietates du cosmos, qui dépasse la multiplicité de l'information des originalia, des sententiae, des magistralia qui s'y trouvent rassemblés. Pour l'érudit d'aujourd'hui qui dénoue avec peine les fils entrelacés des citations, il révèle une trame faite d'échanges vivaces entre Robert Grosseteste, Alexandre de Halès, le mystérieux experimentator, ou encore Arnold de Saxe, qui voyagent parmi les centres savants d'Angleterre, de France et d'Allemagne. Il livre aussi un héritage séculaire de notions enseignées depuis la fin de l'Antiquité et confrontées aux premiers apports scientifiques du monde arabe et des premiers astronomes ou lecteurs de la Perspectiva du monde latin, comme Raymond de Marseille ou Robert Grosseteste.

\section{Réception du livre VIII}

\section{A. Notes marginales destinées à la prédication}

La question reste discutée de savoir si les notes marginales qui parsèment les copies de certains des livres du De proprietatibus rerum ont été initialement pensées et rédigées par Barthélemy l'Anglais lui-même, ou s'il s'agit d'un tout premier indice d'exploitation de la 
compilation naturelle à des fins de prédication. Quoi qu'il en soit, il est certain que Barthélemy avait destiné son œuvre à des fins d'édification via la pastorale, comme un instrument de travail facilitant la rédaction de sermons par ses frères mendiants. Que les notes soient tout à fait contemporaines de l'écriture du De proprietatibus, ou légèrement postérieures, elles témoignent directement de l'utilitas de la compilation sur les propriétés des choses.

La logique qui sous-tend la moralisation du livre VIII est de nature hiérarchique. Une telle conception permet au glosateur de créer des analogies systématiques entre la sphère céleste et la hiérarchie des dignités terrestres (ecclésiastiques et laïques). Les éléments structurants sur lesquels il s'appuie sont assez divers. Chaque fois que le texte évoque des nombres, l'annotateur crée une glose morale numérologique (par exemple: les douze parts du Zodiaque sont mises en rapport avec les douze apôtres ${ }^{238}$; les quatres signes cardinaux du Zodiaque sont interprétés comme les quatre évangélistes ${ }^{239}$ ). Des réseaux sémantiques se tissent également : l'axe du monde, la sphère dans sa totalité, Jupiter, le Soleil, la lumière sont associés au Christ ${ }^{240}$, le signe de la Vierge, la Lune, l'étoile polaire à la Vierge ${ }^{241}$, l'auteur des notes négligeant les propriétés négatives de la Lune; les rayons du Soleil, en nombre de douze selon Martianus Capella, les signes du zodiaque et les étoiles fixes sont rapportés aux apôtre ${ }^{242}$; les constellations sont chacune reliées à un groupe ecclésiastique, Arcturus à l'Église ${ }^{243}$, Orion aux martyrs ${ }^{244}$, les Hyades aux confesseurs $^{245}$, les Pléiades aux saints ${ }^{246}$, Syrius, étoile des jours caniculaires, à l'Antichrist ${ }^{247}$ et aux hérétiques ${ }^{248}$; Saturne évoque le diable ${ }^{249}$. D'autres noms ou adjectifs fonctionnent sur ce même principe analogique : la matrix entraîne une note sur l'ecclesia mater $^{250}$, l'adjectif vilis fait place à une note sur les humiles ${ }^{251}$, tout ce qui est superior, nobilior, celestis appuie une opposition des catégories contemplatives aux couches actives de la société. Le phénomène des éclipses, qui implique la relation entre le Soleil/ Christ et la Lune/ Vierge, est rapporté aux nœuds lunaires qui sont identifiés aux deux personnages bibliques, antithétiques au Christ par leur fonction: le diable et son comparse eschatologique, l'Antichrist ${ }^{252}$. La théorie de l'influence du monde céleste sur les réalités inférieures, que traduisent des mots comme effectus (26 occurrences dans le texte de Barthélemy) a pour pendant des notes qui rappellent l'effet que tel groupe ecclésiastique ou tel sentiment religieux peut avoir sur le monde ici-bas (18 occurrences dans les notes marginales). Le réflexe de s'accrocher de cette manière à toute typologie fournie par le texte convainc que le rédacteur a cherché à utiliser chaque énumération de composantes d'un ensemble (signes du zodiaque, planètes, types de cercles célestes) pour établir des relations, métonymiques et synecdotiques, entre une catégorie socio-morale ou religieuse et un élément céleste. Un double jeu est donc à l'œuvre, avec la signification globale impartie à telle ou telle composante céleste, d'une part, et avec les différentes propriétés qui lui sont associées, de l'autre.

Cette volonté de rapprocher la condition de l'Église des réalités célestes est expressément formulée par la note suivante: Nota quod tota ecclesia disponitur et ordinatur secundum celestia (VIII.22, 1. 136/ n. 41). Elle se traduit aussi par la récurrence des analogies faites avec le Christ (61 occurrences), avec les prélats (60 occ.) associés aux religieux pris globalement (33) et aux différents ordres - qu'ils soient prêcheurs (10) ou cisterciens (1) -, avec l'Église (58), avec les notions de grâce (48) et de charité (20). Cette dernière vertu théologale très investie par les franciscains est le plus souvent mentionnée, à la différence de la foi (2 occurrences) et de l'espérance (1). L'ensemble de la société est divisé par l'entremise de plusieurs relations à caractère holistique, entre groupes binaires 
pour la plupart, comme celle qui existe entre bons et méchants (on repère 57 références à la catégorie des boni), entre clercs et laïcs ou entre prélats et fidèles. À l'intérieur de chaque groupe, la catégorie censée être supérieure (les bons, les clercs, les prélats) joue le rôle de modèle pour l'autre. Par ailleurs, l'analogie qui est affirmée par 23 notes marginales entre l'Église et l'âme rend toutes les moralisations ecclésiologiques transférables au domaine de la psychologie.

Tradition et innovation en matière d'allégorie caractérisent les notes moralisatrices. Au rapprochement, déjà fait à l'âge de la patristique, entre le ciel aqueux et les anges ${ }^{253}$ sont associées des correspondances qui se font l'écho des nouveaux offices instaurés par la papauté, tels que le subdélégué apostolique, office créé sous Innocent III ${ }^{254}$, et par la royauté française, tels que les baillis et officiers, dont le rôle se précise après $1240^{255}$. Elles rappellent la distinction, née au XII ${ }^{\text {e }}$ siècle ${ }^{256}$, entre les temporalia et les spiritualia qui tous deux ont pour source le Christ ${ }^{257}$. Selon l'énoncé de deux notes marginales, les temporalia entraînent la perte des biens spirituels ${ }^{258}$ et entravent l'accès aux biens éternels ${ }^{259}$. En lien direct, la critique de l'amour des biens du monde ici-bas est répétée à plusieurs reprises ${ }^{260}$ , et ne manque pas de prendre pour cible les représentants des seigneurs, les baillis, qui sont comparés aux scorpions, aux béliers et aux capricornes ${ }^{261}$. Un réseau large de notes élabore donc l'apologie du mépris ou du détachement des biens temporels.

À ces moralisations sociales se mêlent des considérations eschatologiques ${ }^{262}$, des remarques économiques ${ }^{263}$, des phrases à résonance parémiologique ${ }^{264}$. D'autres notes se ressentent des débats scolastiques du milieu du XIII ${ }^{\mathrm{e}}$ siècle, comme la question de la pluralité des formes substantielles ${ }^{265}$.

Signe d'une culture de la moralisation assez diffusée, en particulier chez les franciscains, on retrouve les analogies entre ciel et Église dans un traité classé parmi les œuvres spuria de Bonaventure, l'Opusculum de ecclesiastica hierarchia ${ }^{266}$. Son auteur s'adonne à un long exercice d'interprétation qui rappelle les notes marginales accompagnant le texte de Barthélemy, le terme de ciel recouvrant plusieurs significations: auctoritas iudicum et prelatorum; sanctitas religiosorum sive celestis et sublimis conversatio contemplativorum; circuli celi solstitiales / prelati, equinoctiales / subditi, estivales / contemplativi, hiemales / boni activi et officiati ; doctores Sacre Scripture et predicatores ; Christus ; Virgo ${ }^{267}$.

Les analogies articulées entre le monde céleste et le monde ecclésiastique sont la conséquence d'un effort d'interpréter la société sur la base de ramifications multiples issues d'un système organisé par la volonté divine. Elles s'ajoutent aux hiérarchies légitimées par la structure du corps, dont les membres, de la tête aux pieds, correspondent à telle ou telle dignité de la société ecclésiastique. Alors que d'autres entreprises tropologiques rapportaient au corps la hiérarchie laïque, la série des notes interprétatives qui parsèment le livre VIII du DPR fait du ciel le parangon de la hiérarchie ecclésiastique.

\section{B. Allégorisations, récritures, révisions, gloses : de larges emprunts au De mundo}

157 Best-seller dès le XIII ${ }^{\mathrm{e}}$ siècle, la compilation de Barthélemy l'Anglais a servi abondamment à la rédaction de nouvelles œuvres qui l'ont utilisée comme un dictionnaire ou comme un florilège, sans se soucier toujours de mentionner leur source. À l'instar d'autres livres du $D P R^{268}$, le De mundo et corporibus celestibus a été de même compulsé par plusieurs auteurs jusqu'au XVIII ${ }^{\mathrm{e}}$ siècle. Que ce soit à la lettre ou sous forme de moralisations, le texte de 
Barthélemy l'Anglais a formé une partie significative de la matière première d'encyclopédies postérieures, en passant dans l'Historia naturalis du franciscain Juan Gil de Zamora, dans le Pro conservanda sanitate attribué au franciscain Vital du Four ${ }^{269}$, dans le recueil d'exempla appelé Liber de exemplis et similitudinibus rerum du dominicain Giovanni di San Gimignano, dans le Reductorium morale du bénédictin Pierre Bersuire, dans le Liber de moralitatibus du franciscain Marc d'Orvieto, dans l'anonyme Multifarium ${ }^{270}$. Dans la lignée de la réécriture, il convient de signaler la vaste entreprise d'Alberto Marchesi di Cotignola (†1531), de l'observance franciscaine, qui, dans le Coeliloquium morale, s'adonne à une lecture allégorique de plusieurs livres du DPR dont il reprend le texte. De la sorte, aussi bien les chapitres portant sur la Trinité que sur la nature des cieux, des planètes et des signes du zodiaque se retrouvent agencés dans un nouvel exercice de moralisation ${ }^{271}$.

En-dehors des encyclopédies de la seconde génération et des réécritures et adaptations du DPR dans son ensemble, les textes où nous avons réussi à repérer des citations tirées du livre VIII en particulier relèvent de genres littéraires des plus variés: sermons, compilations à caractère médical, gloses et commentaires, etc. Le De mundo et corporibus celestibus a également servi de répertoire pour composer des notices de dictionnaire.

Dans la littérature homilétique, les premiers indices de l'utilisation du DPR apparaissent dès la fin du XIII ${ }^{\mathrm{e}}$ siècle. C'est le franciscain Servasanto di Faenza, auquel on attribue les Sermones de sanctis publiés parmi les œuvres de Bonaventure, qui serait un des premiers à emprunter les propriétés des corps célestes systématisées par Barthélemy l'Anglais ${ }^{272}$. Pour l'Angleterre, Siegfried Wenzel a signalé l'usage courant du DPR dans l'écriture des sermons au XIV siècle $^{273}$. On voit par exemple John Waldeby faire mention, dans son Ave Maria $^{274}$ composé entre 1355 et 1372, de la définition que donne Barthélemy de l'étoile polaire. À un autre moment, à un autre endroit, Pseudo-Pierre de Palude, identifié par certains à Pierre de Colle, choisit une citation du livre VIII du DPR comme thème d'un sermon prêché à la saint Andrée ${ }^{27}$. À la fin du XV siècle, ce même sermon fut à son tour copié par un moine de Vadstena, Iohannes Suenonis ${ }^{276}$. Bernardino de Busti, prédicateur de l'observance franciscaine, emploie plusieurs extraits du DPR dans son Rosarium sermonum rédigé vers 1490. Dans un très long sermon sur la conscience, Bernardino bâtit l'analogie entre la grâce et la lumière du soleil en répartissant en onze effets les propriétés que Barthélemy associe au Soleil dans le chapitre 17 du livre VIII ${ }^{277}$.

Du XIV ${ }^{e}$ au XVIII ${ }^{e}$ siècle, des théologiens continuent à faire usage des propriétés indexées par Barthélemy. Ainsi, la définition évocatrice de l'imaginaire monastique du contemptus mundi, décrivant le monde comme prison de l'âme, lieu des souffrances, tentations et illusions ${ }^{278}$, apparaît dans le Stimulus conscientiae attribué à Richard Rolle of Hampole (1290-1349) ${ }^{279}$ et sous la plume de Gerhardt Groote (1340-1384) dans le contexte théologique de la devotio moderna ${ }^{280}$ ou encore dans des miscellanées théologiques allemandes $\mathrm{du} \mathrm{XV}$ siècle ${ }^{281}$. Johannes von Paltz (1445-1511) ${ }^{282}$ reprend la définition du ciel empyrée attribuée par Barthélemy à Raban Maur (VIII.4, 1.22-30). Barthélemy de Chasseneux (c.1480-1541), dans le Catalogus glorie mundi, utilise le livre VIII dans une consideratio sur les cieux ${ }^{283}$; le jésuite Louis Ballester (1544-1624) le mentionne à quelques reprises dans les chapitres consacrés dans son Hierologia à la nature des étoiles, à la description des constellations et des planètes ${ }^{284}$, le franciscain Pedro Polo fait souvent référence, dans toutes ses œuvres, au "Minorita Anglicus", dans lequel il faut reconnaître le franciscain Barthélemy l'Anglais ${ }^{285}$.

Dans l'Epistola Augustini de Tridento, lectoris Perusii, ad Nicolaum episcopum Tridentinum, qui se trouve conservée dans un manuscrit de Munich, plusieurs citations attribuées à 
Hippocrate et Galien se rapprochent beaucoup du De mundo et corporibus celestibus, d'où provient probablement aussi la phrase du De iudiciis attribuée à Ptolémée (München, B.S.B., clm 647, f. 4v-5r). Par voie directe ou indirecte, le DPR a été compulsé aussi par Michel Savonarole qui a intégré dans son Speculum physionomie des éléments du livre V relatifs à la description des parties du corps et du livre VIII relatifs aux propriétés des signes zodiacaux et des planètes ${ }^{286}$.

Les chapitres 29-32 que Barthélemy consacre à la lumière ont été souvent utilisés, et cela dès le XIVe siècle, par exemple dans le commentaire latin anonyme du Granum sinapis, poème allemand du XIIIe siècle attribué par certains spécialistes à Maître Echkart ${ }^{287}$, jusqu'au XVIe et XVIIe siècle, lorsqu'un Annibale Rosselli ${ }^{288}$ emprunte entre autres les définitions de lumen, splendor et radius et qu'un Rudolf Goclenius ${ }^{289}$ reprend sous la rubrique lux de son Lexikon la question disputée au sujet de la lumière, que Barthélemy a enchâssée dans le chapitre 29 du livre VIII. Bien que les exposés élaborés sur la nature de la lumière soient nombreux depuis le XIIIe siècle, quatre siècles plus tard, Goclenius préfêre incorporer un abrégé déjà disponible qui mettait en place les principaux enjeux théoriques.

Parmi les indices de la très large réception du De proprietatibus rerum, les gloses sont un terrain très fertile, mais encore très peu exploré, de l'utilisation des informations regroupées par Barthélemy. C'est le cas pour l'ensemble des livres du De proprietatibus rerum, mais, selon le sujet traité, telle partie de l'encyclopédie est privilégiée par ses utilisateurs. Citons en exemple les passages du livre VIII qui se trouvent dans les gloses et commentaires sur la Comédie de Dante rédigés au XIV ${ }^{\mathrm{e}}$ siècle par Pietro Alighieri ${ }^{290}$ et par Benvenuto da Imola ${ }^{291}$.

Les multiples tentatives faites à la Renaissance et à l'âge baroque pour classifier les sciences ou élaborer des encyclopédies du savoir ont encore donné une nouvelle vie au De proprietatibus rerum. Dans la lignée du lullisme, le franciscain Bernard de Lavinheta (†c $.1530)^{292}$, dans un ouvrage qui se veut un vademecum à l'œuvre de Raymond Lulle ${ }^{293}$, systématise l'ensemble des sciences en trois parties: les arts du langage (scientia sermocinalis : grammaire, logique, rhétorique), la science de la nature (scientia naturalis : la philosophie naturelle, métaphysique, théologie), la science morale (scientia moralis: éthique, politique, économie ${ }^{294}$. Subsumée aux mathématiques - classées quant à elles parmi les sciences de la philosophie naturelle -, l'astrologie est divisée par Lavinheta en spéculative et pratique ${ }^{295}$. Les chapitres du DPR portant sur les signes du zodiaque et les propriétés des planètes sont intégrés à l'exposé de l'astrologie spéculative, aux côtés d'extraits provenant des traités d'auteurs tels que Jean de Sacrobosco et Jacques Lefèvre d'Etaples. Pour le versant pratique, Lavinheta emprunte à Barthélemy l'Anglais l'aperçu attribué à Misael sur les forces des maisons et les propriétés des aspects ${ }^{296}$.

\section{Traductions du livre VIII et excerpta}

L'intérêt pour le livre VIII se manifeste aussi via des traductions isolées et, beaucoup plus souvent, par des collectes ou des recueils d'extraits.

Le livre sur le cosmos a connu deux traductions vernaculaires, alors qu'il n'existe pas, pour les mêmes langues, de traduction complète du DPR. Ainsi, il y eut une traduction en gaélique dans la première moitié du $\mathrm{XV}^{\mathrm{e}}$ siècle, conservée dans deux manuscrits ${ }^{297}$. Sous forme de fragment, il a également été transposé en allemand, version que l'on peut lire dans un manuscrit provenant du couvent des Franciscains de Wurzburg ${ }^{298}$ : inc. Moventur 
autem omnes planete duplici motu [c. 22 du De mundo]. Ein ieclich planet het zwen löuf. Expl. daz ist dez planeten hus, der da heizzet Mars.

Par ailleurs, le livre sur les réalités célestes a fait l'objet de nombreuses reprises de compilations diverses et de découpages d'extraits de longueurs variables. La plupart des manuscrits que nous avons repérés, copiés essentiellement au $\mathrm{XV}^{\mathrm{e}}$ siècle, ont ciblé la section des chapitres 9 à 19 notamment en raison de leur contenu astrologique. Avec la dizaine de manuscrits déjà signalés par H. Meyer, le total des miscellanea dans lesquels figurent des extraits du livre VIII du DPR monte à une vingtaine de manuscrits, ce qui dénote une transmission vivace de cette partie de la compilation de Barthélemy.

1. Chantilly, Musée Condé, 322, s. XIV, f. $119 \mathrm{v}-121 \mathrm{v}^{299}$ : Spera secundum Isidorum.. revoluta (VIII.6, 1. 2-4 ; DPR_1601: $381^{300}$ ) ; Illa signa que concordant... Iovem. Sol habet... Saturni. Quilibet planeta... debilitatur. Dicunt quedam... Gemini. Domus anguli... laboris. Si aliquis planeta... Misaelem (VIII.9, 1. 43-56, 61-83, 99-101, 108-114, 123-145, 151-184 ; DPR_1601 : 385-388); Aries secundum Misael ... hominis. Et sicut narrat ... dactionis. Secundum Albumasar ... XXVI et cetera. Cancer est signum ... aeris. Signum Leonis ... Capricornus. Virgo est signum ... locum. Libra secundum Misael ... Virgo. Scorpio secundum Misael ... aeris. Sagittarius secundum Misael ... aer. Capricornus secundum Misael ... Capricorni. Aquarius secundum Misael ... Aquarii. Signum Piscium ...oriuntur (VIII.10, 1. 8-17, 21-43, 46-66, 80-97, 105-117, 123-133, 138-151, 155-169, 177-192, 196-207, 212-226, 232-248; DPR_1601 : 389-395) ; Miscalath in libro suo capitulo : Opus planetarum ... planetarum. Miscalath capitulo IIII ... caloris. Secundum astrologos ... mensis. Planetis assignantur ... occidentem. Directus motus ... non retrogradantur (VIII.11, 1. 37-41, 44-51, 81-102, 134-147, 154-165 ; DPR_1601 : 396-399). Dans ce manuscrit, la lacune du texte de Barthélemy concernant l'exaltation et la chute de Jupiter laisse place à un quiproquo qui provoque un transfert des données concernant la Queue du Dragon vers Jupiter : au lieu de Iupiter erigitur <...>. Caput draconis erigitur in Geminis gradibus III et cadit in Sagittario totidem, on y lit: Iuppiter erigitur in Sagittario 3. gradibus et cadit in Geminis totidem. Capricornus erigitur in Geminis gradibus .3. et cadit in Sagitarrio totidem. Pour le reste, le manuscrit de Chantilly comporte à un seul endroit une leçon correcte, que nous n'avons pas repérée ailleurs dans l'ensemble de la tradition du DPR : à la ligne 138 du chapitre 10, Chantilly donne aereum au lieu d'aqueum.

2. Erfurt, Wissenschaftliche Allgemeinbibl., CA fol. $\mathbf{3 4 6}^{301}$ : Le manuscrit contient plusieurs extraits thématiques tirés du DPR aux f. $5 \mathrm{v}-18 \mathrm{v}$, parmi lesquels des passages du livre VIII (f. 8r-10v, inc. Saturnus est a saturando).

3. Krakow, Biblioteka Jagiellonska, 194 ${ }^{302}$, s. XV, f. 118rb-120ra : Inter XII signa celi... equales. Inter ista... planetarum (VIII.9, 1.16-25, 33-41; DPR_1601: 385); Natura signorum... dictum. Et est signum igneum... Iupiter. De corpore... hominis. Taurus secundum Misael... gradu eius. Ex corpore... vanum. Signum Geminorum... Mercurius. In corpore... Albumasar. Cancer est signum... Mars. Super corpus... tortos. Signum Leonis... nocte. In homine... honorum. Virgo est signum... Luna. Ex parte hominis... locum. Libra secundum Misael... nocte. De corpore... rapine. Scorpius secundum Misael... Mars. Ex parte hominis... ingenii. Sagittarius secundum Misael... Iupiter. Ex parte hominis... sompniorum. Capricornus secundum Misael... Luna. In corpore... sublate. Aquarius secundum Misael... Mercurius. Dominari dicitur... peditis. Signum Piscium... Mars. De corpore hominis... carcerem. Dicitur autem illud... dinoscuntur (VIII.10, 1. 2-4, 9-11, 14-17, $32-34,37-42$, 51-55, 57-63, 80-83, 85-89, 105-108, 110-114, 123-126, 128-133, 138-141, 144-146, 155-158, 160-166, 177-180, 182-188, 196-199, 202-205, 212-215, 217-222, 232-235, 
238-244, 248-269; DPR_1601: 389-396); Ad motum planetarum... corrumpuntur. Diversificatur... naturali. Recollige ergo... completive (VIII.11, 1. 2-29, 42-120, 172-198; DPR_1601 : 396-400) ; Item Saturni circulus... nocivus. In colore... regnum (VIII.12, 1. 10-11, 14-31; DPR_1601 : 400-401) ; Iupiter est planeta... blandus. Hic bonitate... Saturni. Hic adiunctus... Pisces (VIII.13, 1. 4-6, 10-11, 16-25; DPR_1601 : 401-402) ; Mars est planeta... gracilitatem. Secundum Ptholomeum... cursum suum (VIII.14, 1. 3-14, 26-32; DPR_1601 : 402-403) ; Venus qui et Lucifer... ostendit. Ut dicit Martianus... XL diebus (VIII.15, 1. 2-18, 21-34 ; DPR_1601 : 403) ; Mercurius secundum Misael... Sole. Aliquando etiam... dominari. Qui secundum Tholomeum... occultatur quia nunc... graditur. Secundum Tholomeum... Virgine. Sub Mercurio... cursum suum (VIII.16, 1. 2-13, 17-20, 24-33, 36, 40-41, 43-48; DPR_1601 : 404-405) ; Sol quasi solus lucens... dicitur. Bissenos radios... illustrantur. Facit hominem... auri. Sub Sole... veridicus. Cum sit octies... videtur. Velocius quam sagitta... visum nostrum (VIII.17, 1. 2, 56-57, 165-167, 196-200, 205-207, 213-217; DPR_1601 : 405-406 et 408-409) ; Luna quasi luminum una etc ut in proprietatibus habetur. Brevissimum... regni eius. Ut dicit Beda... concentus. Sub Luna... cognovit (VIII.18, 1. 2, 79-84, 139-150, 176-184; DPR_1601: 410-414); Luna ut dicit Tholomeum... carebit. Preterea... caducis (VIII.19, 1. 24-31, 41-55; DPR_1601 : 414-415) ; Stelle secundum Martianum... inferiorum. Item stelle... in quolibet anno (VIII.22, 1. 100-105, 132-140; DPR_1601 : 419-420).

4. München, Bayerische Staatsbibliothek, Clm $667^{303}$, s. XV, f. 38r-40v : Saturnus secundum Mysa... Ptolomeum (VIII.12, 1. 7-38 ; DPR_1601: 400-401) ; Iupiter est benivolus... Martianus. Hic bonitate... Misael (VIII.13, 1. 4-8, 10-37 ; DPR_1601 : 401-402) ; Mars est calidus... passiones. Sub Marte... horis (VIII.14, 1. 3-19, 26-32 ; DPR_1601 : 402-403) ; Venus qui et Lucifer... veridicus (VIII.15, 1.2-32；DPR_1601: 403) ; Mercurius secundum Mysa... Soli. Aliquando... amatores. Semper iuxta... malus (VIII.16, 1.2-13, 17-25, 31-46; DPR_1601: 404-405); Sol secundum Isidorum... transcendit. Circulus Solis... gradus. Sub Sole... veridicus. Item est sue... videtur (VIII.17, 1. 2-17, 21-23, 196-200, 203-207; DPR_1601: 405 et 409) ; Luna dicitur quasi... celi (VIII.18, 1. 2, 4-19; DPR_1601: 410).

5. Oxford, Bodl. Libr., Saville $17^{304}$, s. XV, f. 22v-46r: Inc. Spera celi est, ut dicit Isidorus... ad nonas septembris et sic sunt L sicut ibi dicitur (VIII.6-VIII.28 ; DPR_1601 : 381-425).

6. Paris, B.n.F. lat. $6957^{305}$, s. XV, f. $158 \mathrm{v}-161 \mathrm{r}$ : Zodiachus est circulus oblicus... signum unum (VIII.9, 1. 2-11 ; DPR_1601 : 384-385). Naturas igitur singulorum... assumentes. Aries igitur est signum igneum... nativitatis hominis. Taurus est signum terreum... dationis. Gemini est signum aereum... somniorum. Cancer est signum aqueum... hereditatis. Leo est signum igneum... vestimentorum. Virgo est signum terreum... ad locum. Libra est signum aqueum... rapine. Scorpio est signum aqueum... ingenii. Sagittarius est signum igneum... honoris. Capricornus est signum terreum... furti. Aquarius est signum masculinum... substantie. Pisces est signum aqueum ... tristicias. Explicit tractatus de signis, Deo gratias (VIII.10, 1. 2-3, 8-17, 32-43, 51-63, 80-92, 105-114, 123-133, 138-146, 155-166, 177-187, 196-205, 212-222, 232-243; DPR_1601: 389-395); Has itaque XII signorum celi propritates... dicenda. Hii enim planete... exaltantur. Et ideo de eorum proprietatibus aliqua sunt dicenda ad Saturnum exordium sumantia. Saturnus enim est planeta malivolus... tristem. Iupiter est planeta benivolus... rotundam. Mars est planeta calidus... siccitatis. Sol est sic dictus... animam. Venus est planeta benivolus... cantu. Mercurius est planeta temperatus... calculi. Luna dicitur quasi luminum... occulorum. Et hec sint dicta de proprietatibus planetarum (VIII.10, 1. 253-271 ; VIII.11, 1. 80-117 ; VIII.12, 1. 7-9, 16-21 ; VIII.13, 1. 3-5, 16-22 ; VIII.14, 1. 2-14 ; VIII.17, 1. 2-8, 197-199 ; VIII.15, 1. 2-7, 23-24 ; VIII.16, 
1. 2-6, 24-26 ; VIII.18, 1. 2, 162-165 ; VIII.19, 1. 23-29 ; DPR_1601 : 395-398, 400-405, 409-410, 413 et 415$)$.

7. Praha, Národní knihovna České republiky, I.G.6 ${ }^{306}$, f. 270r-274r: Galaxia est circulus... manifestior. Item Aristoteles... orbis (VIII.8, 1. 2-10, 25-29; DPR_1601: 384); Zodiacus est circulus... designata. Sunt autem hec signa... sicut Aries etc. In quibus signis... operantur. Planeta igitur... fortior est. Secundum autem istorum... in suo genere (VIII.9, 1. 2-7, 11-12, 26-61, 93-101, 115-121; DPR_1601: 384-387) ; Aries est signum... hominis. Albumasar... donationis. In primo gradu... aerem. Cancer est signum... aeris. Leonis signum... Capricornus. Virgo est signum... ad locum. Libra est signum... Virgo. Scorpius est signum... serenitas. Sagittarius est signum... aeris perturbatio. Capricornus est signum... occidit. Aquarius est signum... Capricornus. Piscis est signum... Aquarii (VIII.10, 1. 4, 8-17, 22-43, 47-65, 80-97, 105-117, 123-133, 138-151, 155-169, 177-191, 196-207, 212-225, 232-248, 259-269; DPR_1601 : 389-396) ; Moventur autem planete... firmamenti. Diversificantur ... in malo. Domus Saturni ... in medio. Sunt autem iste stelle... Albumasar (VIII.11, 1. 2-6, 42-54, 110-161, 175-189; DPR_1601 : 396-400) ; Saturnus est planeta... procedendo. Duas habet... Ptolomeum (VIII.12, 1.7-13, 16-38; DPR_1601： 400-401); Iupiter est benivolus... posuerunt. Hic bonitate... coniunctus. Hic adiunctus... Ptolomeum (VIII.13, 1. 4-7, 10-12, 16-37 ; DPR_1601 : 401-402) ; Mars est calidus... passiones. Sub Marte... dolosus (VIII.14, l. 2-19, 26-31 ; DPR_1601 : 402-403) ; Venus qui et Lucifer... temperatur. Semper comitatur... serena. Quando Venus... econverso. Malitiam reprimit... veridicus (VIII.15, 1. 2-4, 9-15, 18-19, 22-32 ; DPR_1601 : 403) ; Mercurius est calidus... Sol. Dicitur autem... amatores. Semper iuxta... malus (VIII.16, 1.2-13, 23-25, 31-46; DPR_1601: 404-405); Sol totius luminis... inferiora, masculinus... siccus. Maior est... multiplicitate. Item Aristoteles... illustrantur. Vim habet... effectibus. Item temporum... pallet. Unde Beda... significat. Solis constellatione... fugat. Item que qualitatis ... videtur. Sub Sole... veridicus (VIII.17, 1. 3-4, 6-7, 9-12, 51-57, 125-135, 139, 145-147, 151-157, 162-173, 204-207, 196-200 ; DPR_1601 : 405-409) ; Luna dicitur luminum una et est planeta... nocturnus. Vim habet... ros. Item cum stellis... malivola. Benivoli sunt... bonus. Item est mutationis... futura. Secundum Ptolomeum... humiditatis. Item secundum astrologos... operatur. Unde Galienus sperandum est (VIII.18, 1. 2, 84-85, 72-76, VIII.19, 1. 9-10, VIII.11, 1. 70-75, VIII.18, 1. 138-145, VIII.19, l. 24-32, VIII.18, 1. 173-178, 187-208 ; DPR_1601 : 410, 414, 397, 413, 415 et 413-414).

8. Sheffield, Central Library, Jackson Collection $\mathbf{1 3 0 2}^{307}$, s. XVe-XVI ${ }^{e}$, f. $74 r-81 v$ : Zodiacus est circulus... auctor (VIII.9, 1.2-183; DPR_1601: 384-388); Naturas autem singulorum... Ysidorus. Nota quod secundum... agere dicuntur (VIII.10, 1.2-252, 259-269; DPR_1601 : 389-396); Moventur omnes planete... completive (VIII.11, 1.2-198; DPR_1601: 396-400) ; Sol secundum Ysidorum... sufficiant (VIII.17, 1. 2-218; DPR_1601 : 405-409) ; Luna dicitur quasi luminum... permanet (VIII.18, 1. 2-23 ; DPR_1601 : 410).

9. Venezia, Biblioteca Nazionale Marciana, lat. VIII, 69 (3349) ${ }^{308}$, daté de 1488 , f. 1r-11r : Introductorium astronomicum. Spera secundum Isidorum... revoluta (VIII.6, 1. 2-4; DPR_1601 : 381) ; Illa signa que concordant... Iovem. Sol habet... Saturni. Quilibet planeta... debilitatur. Dicunt quedam... Gemini. Domus anguli... laboris. Si aliquis planeta... Misaelem (VIII.9, 1. 43-56, 61-82, 98-100, 107-113, 122-144, 150-183; DPR_1601 : 385-388) ; Aries secundum Misaelem... hominis. Et sicut narrat... dactionis. Secundum Albumasar... XXVI et cetera. Cancer est signum... aeris. Signum Leonis... Capricornus. Virgo est signum... locum. Libra secundum Misaelem... Virgo. Scorpio secundum Misaelem... aeris. Sagittarius secundum Misaelem... aer. Capricornus secundum Misaelem... Capricorni. Aquarius secundum Misaelem... Aquarii. Signum Piscium... 
oriuntur (VIII.10, 1. 8-17, 21-43, 46-66, 80-97, 105-117, 123-133, 138-151, 155-169, 177-192, 196-207, 212-226, 232-248; DPR_1601: 389-396); Miscalath dicit suo capitulo: Opus planetarum... planetarum. Miscalath capitulo IIII... caloris. Secundum astrologos... mensis. Planetis assignantur... occidentem. Directus motus... non retrogradantur. Beda in libro... differentes (VIII.11, 1.37-41, 45-51, 81-102, 134-147, 154-165, 172-174 ; DPR_1601 : 396-399) ; Saturnus est secundum Misaelem... Ptolomeus (VIII.12, 1. 7-38; DPR_1601: 400-401) ; Iupiter planeta... Martianus. Hic adiunctus... Misael (VIII.13, 1. 3-8, 16-37; DPR_1601 : 401-402) ; Mars est planeta... vindicte. In colore... Martianus. Hic planeta... dolosus (VIII.14, 1.2-6, 9-10, 12-31; DPR_1601 : 402-403); Venus planeta... temperatus. Colorem habet... fulget. Ex parte corporis... 368 (VIII.15, 1.2-4, 11-13, 23-34; DPR_1601 : 403) ; Mercurius secundum Misaelem... efficiatur. Secundum Ptolomeum... mercatorum. Secundum Ptolomeum... malus (VIII.16, 1.2-6, 24-27, 40-46; DPR_1601: 404-405) ; Sol secundum Messealath... luminis. Ambitur in Exameron... firmamenti. Dionysius in libro... vivificat. Sol etiam, ut dicit Beda... Beda. De hoc dicit... disponit. Sub Sole... veridicus (VIII.17, 1. 5-11, 34-42, 58-64, 151-157, 165-169, 196-200; DPR_1601 : 405-406 et 408-409); Sicut dicit Ptolomeus... cursum suum. Beda si Luna... pretendit. Ptolomeus in libro... operatur (VIII.18, 1.82-87, 139-143, 175-178; DPR_1601 : 411-413) ; Luna ut Ptolomeus... carebit. Dicitur in libro... sociata (VIII.19, 1.24-31, 34-51; DPR_1601 : 415); Invisibiliter enim operatur... animatum (VIII.29, 1. 98-103; DPR_1601 : 427).

Le texte de ce manuscrit, qui a appartenu aux Capucins de Venise, est identique à celui de Chantilly, décrit plus haut, à la différence qu'il contient quelques extraits supplémentaires. Les deux manuscrits remontent probablement à un modèle commun ${ }^{309}$.

10. Zurich, Zentralbibliothek, C $\mathbf{1 5 0}^{310}$, s. XV : le manuscrit comporte plusieurs traités fondés sur les livres VIII (f. 1r-9r), IX (f. 9r-12r) et XVII (f. 114r-130v) du DPR. Aux f. 1r-9r : Incipit de celo, de stellis et temporibus tractatus, les passages extraits du livre VIII sont complétés à deux endroits par des citations du De natura rerum d'Isidore de Séville : Adiuvante gratia Christi proprietates celi aliquas breviter annotemus. Celum angelorum... actualitatem. Alexander sic... luminosum. Firmamentum itaque vocatur... sperica est (VIII.2, 1. 4-29, 37-51, 113-117 ; DPR_1601 : 372-374) ; Sextum celum est... suspense (VIII.3, 1. 2-10 ; DPR_1601 : 378) ; Celum empyreum est... propinquum. Et licet Deus... operatur (VIII.4, 1. 2-9, 30-35 ; DPR_1601 : 379-380) ; Ether grece... sive lucis. Et continet... immutentur (VIII.5, 1. 2-5, 37-45 ; DPR_1601 : 380-381) ; Spera celi... circumfertur. Axis est quedam... nuncupatur. Spera autem motus... superatur (VIII.6, 1. 12-24, 33-45, 55-67 ; DPR_1601 : 382-383) ; Circuli celi... imperfecti (VIII.7, 1. 2-30 ; DPR_1601 : 383) ; Galaxia est... manifestior (VIII.8, 1. 2-10 ; DPR_1601 : 384) ; Zodiacus circulus... immorentur. Signa ista... representent (VIII.9, 1. 2-5, 11-15 ; DPR_1601 : 384-385) ; Aries est signum... Martis. De corpore... longum. Taurus secundum Misael... Veneris. Ideo dicitur... agriculture. Corpori... sulcando. Cancer est signum... Lune. Super corpus... hereditatibus. Dicitur autem Cancer... retrogadationem. Signum Geminorum... Mercurii. In corpore... sompniorum. Isti Gemini... geminatur. Signum Leonis... Solis. In homine honorum. Dicitur autem Leo... Mercurii. Ex parte hominis... Veneris. De corpore... rapine. Dicitur ab instrumento... Martis. Ex parte hominis... ingenii. Dicitur autem signum... Caude. Ex parte hominis... sompniorum. Dicitur autem Sagittarius... domicilium. In homine... sublate. Dicitur Capricornus... Saturni. Dominari... peditis. Dicitur autem Aquarius... tempore (VIII.10, 1. 4-10, 14-17, 32-33, 43-46, 37-44, 80-81, 85-92, 97-100, 51-53, 57-63, 67-71, 105-106, 110-114, 117-124, 128-139, 144-146, 151-156, 160-166, 170-178, 182-188, 192-198, 202-205, 207-213, 217-222, 226-231 ; DPR_1601 : 389-395) ; Saturnus est a saturando... futura erit (section qui abrège et parfois réorganise les renseignements du VIII.12, 1.2-VIII.18, 1.145;DPR_1601 : 400-413) ; Et nota 
quod hii VII planete... salubris (VIII.11, 1. 80-95; DPR_1601: 397-398) ; Cometa est stella... Crisostomus (VIII.21; DPR_1601 : 417) ; Polus... invisibilis. Axis... intellectualis. Polus... intuetur (VIII.23, 1. 2-9, 13-16, 21-26 ; DPR_1601 : 421) ; Arcturus... vocatur. Apparent... centrum earum. Inter medias... prenuncians (VIII.24, $12-13,18-23$, 35-39 ; DPR_1601 : 422) ; Orion... generativus. Est autem constellatio... pulcritudinis. Nam splendor... Thauro (VIII.25, 1. 2-4, 10-12, 20-34 ; DPR_1601 : 423); Hyades... ordinatas (VIII.26, 1.2-17; DPR_1601 : 424); Plyades... poetarum (VIII.27, 1.2-14 ; DPR_1601: 424) ; Canicula... periculosum. Durant... septembris (VIII.28, 1. 2-6, 23-24 ; DPR_1601 : 425) ; Lux... substantiam (VIII.29, 1.2-22 ; DPR_1601 : 425-426) ; Radius... actionem (VIII.32, 1.2-4 ; DPR_1601 : 431); Umbra... luminosum (VIII.33, 1.2-3 ; DPR_1601 : 432) ; Tenebra... privatio (VIII.34, 1. 2-4 ; DPR_1601 : 433).

11. Zwickau, Ratsschulbibliothek, Ms. I.XV.4 ${ }^{311}$, s. XV. Aux f. 60ra-82rb, on trouve un compendium réunissant des extraits des livres VIII (f. 60ra-67va), IX (f. 67vb-74va), VIII (74va-75rb), X (f. 75va-76ra), XI (f. 76rb-78vb), XIII (f. 78vb-79ra), XIV (f. 79ra-vb) et IV (f. 79vb-82rb) du DPR. Voici les passages empruntés au livre VIII : Mundus itaque ut dicit Mercurius... laudandus. Nam illa pars... Aristotelem. Tanto enim mundus... sufficiant (VIII.1, 1. 2-125, 129-142, 166-207; DPR_1601: 367-372) ; Nunc ad scribendas... infinitum. Nota quod partes celi... conformat (VIII.2, 1.2-71, 225-248; DPR_1601: 372-373 et 377-378); Sextum celum... Alexander (VIII.3, 1.2-70 ; DPR_1601 : 378-379) ; Celum empureum... operatur (VIII.4, 1. 2-35; DPR_1601: 379-380); Ether grece... contraxisset. Secundum Macrobium... immutentur (VIII.5, 1.2-25, 35-45; DPR_1601 : 380-381) ; Spera celi... Ysidorus (VIII.6, 1. 2-46 ; DPR_1601 : 381-382) ; Naturas autem signorum... Aries est signum masculinum... Martis. De corpore... astrorum. Thaurus secundum... Veneris. Ex corpore... collo (VIII.10, 1. 2-4, 9-10, 14-22, 32-33, 37-38; DPR_1601 : 389-396); in colore... Martianus (VIII.13, 1. 5-37 ; DPR_1601 : 401-402) ; Mars deus... cursum suum (VIII.14, 1.2-32 ; DPR_1601 : 402-403); Venus qui et Lucifer... temperatus. Inter omnia... diebus (VIII.15, 1.2-4, 12-34; DPR_1601 : 403) ; Mercurius secundum... circulus. Unde secundum... post. Secundum autem... Tholomeus (VIII.16, 1. 2-14, 24-36, 40-48; DPR_1601 : 404-405) ; Sol secundum... illuminatur. De hiis... sufficiant (VIII.17, 1. 2-54, 58-218 ; DPR_1601 : 405-409) ; Luna dicitur... Macrobius. Item Luna... cursum suum. Item Luna... Beda. Item Luna... ministrativa. Item Luna... Macrobius. Item Luna... iudicandum est (VIII.18, 1. 2-25, 48-87, 138-145, 151-152, 162-166, 173-209; DPR_1601: 410-414) ; Nam ut dicit... caducis (VIII.19, 1.23-55; DPR_1601: 414-415); Stelle a stando... previdentur. Nam secundum Alfraganum... algorem. Nam stelle... sumit. Item secundum... planetarum (VIII.22, 1. 2-6, 12-36, 50-73, 106-116; DPR_1601 : 417-420); Lux ut dicit Basilius... invenitur. Preterea si lux... corporibus (VIII.29, 1. 2-18, 33-209; DPR_1601 : 425-430); Splendor est quidam... Basilius (VIII.30, 1. 2-10; DPR_1601 : 430) ; Lumen differt... invenitur (VIII.31, 1.2-48; DPR_1601: 430-431); Nota quod radius... Basilius (VIII.32, 1. 2-4 ; DPR_1601 : 431) ; Tenebra est... nutrit (VIII.34, 1. 2-8 ; DPR_1601 : 433) ; Zodiacus est circulus... de aliis. Secundum autem signorum... laboris (VIII.9, 1. 2-63, 115-144; DPR_1601 : 384-388). Le manuscrit accuse une lacune d'un feuillet entre le f. 62v et le f. 63r correspondant aux chapitres 10, 1. 38-12 du livre VIII du DPR. 


\section{BIBLIOGRAPHIE}

\section{Éditions de textes}

ABBON DE FLEURY, De ratione spere, THOMSON Ron B. (éd.), « Two Astronomical Tractates of Abbo of Fleury ", in NORTH John D. et ROCHE John J. (éd.), The Light of Nature. Essays in the History and Philosophy of Science presented to A. C. Crombie, Dordrecht, 1985 (Archives internationales d'histoire des idées, 110), p. 113-133.

AL-BATTĀNII, Albatenii Opus astronomicum ad fidem codicis Escurialensis arabice editum, latine versum, adnotationibus instructum, NALLINo Carlo Alfonso (éd.), Milan, 1899-1907.

AL-BITRŪJI, De motibus celorum, Critical edition of the Latin Translation of Michael Scot, Berkeley, CARMODY Francis J. (éd.), Los Angeles, 1952.

AL-FARGHĀNI, Differentie scientie astrorum, CARMODY Francis J. (éd.), Berkeley, 1943.

AL-FARGHĀNI, Il 'Libro dell'aggregazione delle stelle' secondo il codice mediceo-laurenziano Pl. 29. cod.9, CAMPANi Romeo (éd.), Città di Castello, 1910.

ALANUS DE INSULIS, Hierarchia, in Alain de Lille. Textes inédits avec une introduction sur sa vie et ses œuvres, D’ALVERNY Marie-Thérèse (éd.), Paris, 1965, p. 219-235.

ALBERTO MARCHESI DI COTIGNOLA, Coeliloquium morale, Bologna, 1529.

ALBERTUS MAGNUS, Summa de creaturis, BORGNET Auguste (éd.), Opera omnia Alberti Magni, t. 34, Paris, 1895.

AlBertus magnus, De homine, Borgnet Auguste (éd.), Opera omnia Alberti Magni, t. 35, Paris, 1896.

ALBERTUS MAGNUS, De homine, ANZULEWICZ, SÖDER,J. R., (éd.), Münster, 2008 (Albertus Magnus, Opera omnia, 27, pars II).

ALEXANDRI DE HALES, Summa Theologica seu 'Summa fratris Alexandri' lib. III, DOUCET Victorin (éd.), Quaracchi, 1948

ALHACEN (IBN AL-HAYTAM), De aspectibus : SMITH, Mark A., Alhacen's Theory of Visual Perception, t. 1, Philadelphia, 2001.

AL-QABĪSII (ALCABITIUS), The Introduction to Astrology, Editions of the Arabic and Latin texts and an English translation, BURNETT Charles, YAMAMOTO Keiji, yANo Michio (ed.), London, 2004.

AUGUSTINUS, Commentaire sur la Genèse au sens littéral, in CEuvres de saint Augustin. La Genèse au sens littéral, AGAËsSE Paul, solignaC Aimé (trad.), Paris, 1972.

AUGUSTINUS, De doctrina christiana, MARTIN Joseph (éd.), Sancti Aurelii Augustini De doctrina christiana, De vera religione, Turnhout, 1962 (CC, SL, 32).

AVICENNA, Liber de anima seu sextus de naturalibus, VAN RIET Simone (éd.), Louvain-Leiden, 1972.

Averrois Cordubendis Commentarium magnum in Aristotelis De anima libros, CRAWFORD Stuart F. (éd.), Cambridge (MA), 1953 (Corpus commentariorum Averrois in Aristotelem, Versio latina, 6,1). 
AVERROES, Commentarium medium in Aristotelis 'De generatione et corruptione' libros, FOBES Francis H., KURLAND Samuel (éd.), Cambridge (MA), 1956.

AVERROES, Compendium libri Aristotelis De sensu et sensato : Averrois Cordubensis Compendia librorum Aristotelis qui 'Parva Naturalia' Vocantur. Compendium libri Aristotelis De sensu et sensato, SHIELDS Emily L., BLUMBeRg Harry (éd.), Cambridge (MA), 1949 (Corpus Philosophorum Medii Aevi, Corpus Commentariorum Averrois in Aristotelem series - versio Latina, VII).

BARTHÉLEMY L'ANGLAIS, De proprietatibus rerum, t. 1, introd. B. VAN DEN ABEELE, Prohemium, éd. H. MEYER, Liv. I, éd. M. W. TWOMEY, Liv. II, éd. B. ROLING, Liv. III et IV, éd. R. J. LONG ; t. 6, Liv. XVII, éd. I. VENTURA, Turnhout, Brepols, 2007 (De diversis artibus).

[BARTHÉLEMY DE CHASSENEUX], Catalogus gloriae mundi, Lyon, 1529.

BEDA, De natura rerum, XV, JONES C. W. (éd.), Turnhout, 1975 (CC, SL, 123 A).

BENEVEnUti de RAmbaldis DE IMola, Comentum super Dantis Aldigherij Comoediam, LACAITA James Philip (éd.), Firenze, 1887, vol. IV.

BERNARD DE LAVINHETA, Practica compendiosa artis Raymundi Lullii, Lyon, 1523.

BonaVentura, Commentarium in secundum librum Sententiarum, in Opera omnia, Collegium a S. Bonaventura (éd.), Quaracchi, 1885.

BONAVENTURA, Commentarium in tertium librum Sententiarum, in Opera omnia, Collegium a S. Bonaventura (éd.), t. 3, Quaracchi, 1887.

BONAVENTURA, Commentarium in quartum librum Sententiarum, in Opera omnia, Collegium a S. Bonaventura (éd.), t. 4, Quaracchi, 1889.

BONAVENTURA, De ecclesiastica hierarchia, in Opera omnia sancti Bonaventurae, PELTIER AdolpheCharles (éd.), Paris, 1866, t. 7, p. 437-497.

BONAVENTURA, Sermones de sanctis, in Sancti Bonaventurae Opera omnia, PELTIER Adolphe-Charles (éd.), t. 14, Paris, 1868.

BURNETT Charles, PINGREE David (éd.), The Liber Aristotilis of Hugo de Santalla, London, 1997

(Warburg Institute Surveys and Texts, 26).

Catalogus gloriae mundi, Lyon, 1529.

Chartularium universitatis Parisiensis, DENIFLE Heinrich, chATELAIN Emile (éd.), Paris, 1889.

DANIEL DE MORLEY, Philosophia mundi, sUdHOFF Karl (éd.), Archiv für Geschichte der Naturwissenschaften und Technik, 8, 1917, p. 6-40.

Das Decretum gelasianum de libris recipiendis et non recipiendis, voN DOBSCHÜTZ Ernst (éd.), Leipzig, 1912.

De potentiis anime et obiectis : CALlus, D. A. (éd.), « The Powers of the Soul. An Early Unpublished Text », Recherches de théologie ancienne et médiévale, 19, 1952, p. 131-170.

Étymologies, livre III. De mathematica, GASPAROTTO Giovanni (éd.), GUILLAUMin Jean-Yves (trad.), Paris, 2009 (Belles-Lettres).

GÉRARD GROTE, Epistolae : Gérard Grote, fondateur de la Dévotion Moderne : Lettres et traités, ÉPINEYBURGARD Georgette (éd.), Turnhout, 1998.

GÉRARD GROTE, Epistolae : Gerardi Magni. Epistolae quas ad fidem codicum recognovit, annotavit, MULDER Willelm (éd.), Antwerpen, 1933. 
Gerardi Magni. Epistolae quas ad fidem codicum recognovit, annotavit, MULDER Willelm (éd.), Antwerpen, 1933.

Glossa ordinaria, in Biblia latina cum glossa ordinaria. Facsimile Reprint of the Editio Princeps Adolph Rusch of Strassburg 1480/81, Turnhout, 1992.

Glossa ordinaria, manuscrit Orléans, Bibliothèque municipale 32 (29).

Guillelmus de Conchis, Glosae super Platonem, JEAUnEAU, E. (éd.), Turnhout, 2006 (CC, CM, 203). HUGO DE SANCTO-CARO, Postilla super totam Bibliam, manuscrits Orléans, Bibliothèque municipale 28 (25) ; Reims, Bibliothèque municipale, 164.

JEAN DE LA ROCHELLE, Tractatus de divisione mutiplici potentiarum animae. Texte critique avec introduction, notes et tables, MICHAUD-QUANTIN Pierre (éd.), Paris, 1964 (Textes philosophiques du Moyen Âge, 11).

JOHANNES VON PALTZ, Opuscula, BURGER Christopher (éd.), Berlin, 1989.

KIBRE Pearl (éd.), Hippocrates latinus, New York, 1985.

Latin Sermon Collections from Later Medieval England, wENZEL Siegfried (éd.), Cambridge, 2005.

Lexikon Philosophicum (...) Informatum opera \& studio Rodolphi Goclenii (...), Frankfurt, 1613.

Les registres de Grégoire IX, AUVRAY Lucien (éd.), Paris, 1896.

[LOUIS BALLESTER], Hierologia sive de sacro sermone, continens summam atque compendium positivae theologiae, Lyon, 1617.

Macrobe. Commentaire au songe de Scipion, ARMISEN-MARCHETTI Mireille (éd.), Paris, 2001

MICHAEL SCOTUS, The Liber Introductorius of Michael Scot, EDWARDS Glenn M. (éd.), Ph.D. Diss., Univ. of Southern California, 1978. [édition du Prohemium].

MORRIN Margaret J., John Waldeby, O.S.A., c. 1315-c. 1372. English Augustinian Preacher and Writer, with a Critical Edition of His Tract on the " Ave Maria », Rome, 1975.

PEDRO POLO, Mansiones encomiasticae adaptatae quadraginta duobus iuxta Evangelium Matthaei progenitoribus Christi Domini, Madrid, 1737.

PEDRO POLO, Mansiones festaque Hebraeorum, Barcelone, 1725.

PEDRO POLO, Mansiones morales, Madrid, 1737.

PEDRO POLO, Mansiones mysticae et asceticae ad fratres, et moniales, Madrid, 1737.

Petra Alleghieri super Dantis ipsius genitoris Comoediam Commentarium, NANNUCCI Vincentio (éd.),

Firenze, 1846.

PLINE, Historia naturalis, BEAUJEU Jean (éd.), Paris, 1950 (Les Belles-Lettres).

PSEUDO-PTOlomaeus, Proportiones, éd. in JUSTE, D., Les Alchandreana primitifs. Étude sur les plus anciens traités astrologiques latins d'origine arabe ( $X^{e}$ siècle), Leiden, 2007 (Brill's Studies in Intellectual History, 152).

PTolomaeus, Tetrabiblos, RoBbins Frank E. (éd.), Cambridge (MA), 1940.

Pymander Mercurii Trismegisti cum commento fratris Hannibalis Rosseli calabri, ordinis minorum regularis observantiae, theologiae \& philosophiae, ad S. Bernardinum Cracoviae professons, liber primussextus, Cracoviae, 1584

RABAN MAUR, De Computo, STEVEnS Wesley M. (éd.), Turnhout, 1979 (CC, CM, 44). 
RAMELli Ilaria (éd.), Tutti i commenti a Marziano Capella, Milano, 2006 (Il pensiero occidentale). RAYMOND DE MARSEILLE, Opera omnia. T. I : Traité de l'astrolabe. Liber cursuum planetarum, D'ALVERNY Marie-Thérèse, BURNETT Charles, POULLE Emmanuel, Paris, 2009.

Remigii Autissiodorensis Commentum in Martianum Capellam, LUTZ Cora E. (éd.), Leiden, 1963 (lib. I-II), 1965 (lib. III-IX).

RICHARD FISHACRE, De luce : In secundum librum Sententiarum. Part 2 : dist. 21-44. Appendices, LONG Robert J. (éd.), München, 2011.

RICHARD ROLLE OF HAMPOLE, Stimulus conscientiae : The Pricke of Conscience (Stimulus conscientiae), A Northumbrian Poem by Richard Rolle de Hampole, MORRIS, Richard, (éd.), Berlin, 1863.

ROBERT GROSSETESTE, Hexaëmeron, DALES Richard. C., GIEBEN Servus, London, 1982.

ROBERT GROSSETESTE, De colore: Gr. DINKOVA-BRUUn, et al. (éd.), The Dimensions of Colour : Robert Grosseteste's De colore, Toronto, 2013 (Durham Medieval and Renaissance Texts, 4). [Edition, translation, and interdisciplinary analysis by Gr. Dinkova-Bruun, E.M. Gasper, M. Huxtable, T. C.B. McLeish, C. Panti, and H. Smithson].

ROBERT GROSSETESTE, De luce, PANTI Cecilia (éd.), in FLOOD, GINTHER, GOERING, Robert Grosseteste and his intellectual milieu, p. 193-237.

ROBERT GROSSETESTE, De sphera, De cometis, de motu supercelestium, PANTI Cecilia (éd.), Moti, virtù e motori celesti nella cosmologia di Roberto Grossatesta. Studio ed edizione dei trattati De sphera, De cometis, De motu supercelestium, Firenze, 2001 (Testi e studi per il Corpus philosophorum Medii Aevi, 16).

ROBERT GROSSETESTE (PS.), Summa philosophiae, BAUR Ludwig (éd.), Die philosophischen Werke des Robert Grosseteste, Bischofs von Lincoln, Münster, 1912 (Beiträge zur Geschichte der Philosophie des Mittelalters 9), p. 275-643.

Rosarium sermonum, Venise, 1498.

SALIMBENE DE ADAM, Cronica, Scalia Giuseppe (éd.), Turnhout, 1998-1999 (CCCM, 125-125A).

schoonheim Pieter L. (éd.), Aristotle's Meteorology in the Arabico-Latin Tradition : A Critical Edition of the Texts, with introduction and indices, Leiden, 2000 (Aristoteles Semitico-Latinus, 12).

Sermones de sanctis, Nuremberg, 1487.

Summae magistri Rolandi Cremonensis O. P. liber tercius, CORTESI Aloys, (éd.) Bergame, 1962.

Thomae Galli Explanatio in libros Dionysii, LAWELL Declan A. (éd.), Turnhout, 2011 (CC, CM, 223).

THOMAS DE AQUINO, Commentum in secundum librum Sententiarum, in Opera omnia, Parma, 1856.

THORNDIKE Lynn (éd.), The Sphere of Sacrobosco and Its Commentators, Chicago, 1949.

\section{Travaux}

ANDERSSON-SCHMITT Margarete, HALLBERG Håkan, HEDLUND Monica, Mittelalterliche Handschriften der Universitätsbibliothek Uppsala, Bd. 2, C 51-200, Stockholm, 1989 ; Bd. 3, C201-300, Stockholm, 1990.

Aujac Germaine, Claude Ptolémée, astronome, astrologue, géographe, Paris, 1993.

Bakhouche Béatrice, Les textes latins d'astronomie : un maillon dans la chaîne du savoir, Leuven, 1996. 
Baur Ludwig, Die Philosophie des Robert Grosseteste, Bischofs von Lincoln, († 1253), Münster, 1917 (Beiträge zur Geschichte der Philosophie des Mittelalters, 18, Heft 4-6).

Beschreibendes Verzeichnis der Amplonianischen Handschriften-Sammlung zu Erfurt, Berlin, 1887.

Bindschedler Maria, Der Lateinische Kommentar zum Granum sinapis, Basel, 1949.

Borrelli Arianna, Aspects of the Astrolabe : 'architectonica ratio' in tenth-and eleventh-century Europe, Stuttgart, 2008.

Borst Arno, Astrolab und Klosterreform an der Jahrtausendwende, Heidelberg, 1989 (Sitzungberichte der Heidelberger Akademie der Wissenschaften, Phil.-hist. Klasse, 1/1989).

Bos Gerrit, Burnett Charles, Scientific Weather Forecasting in the Middle Ages: The Writings of Al-Kindī, London-New York, 2000.

Bultot Robert, "Cosmologie et 'contemptus mundi' », Recherches de théologie ancienne et médiévale. $\mathrm{N}^{\circ}$ spécial I. «Sapientiae doctrina ». Mélanges de théologie et de littérature médiévales offerts à Dom Hildebrand Bascour O.S.B., 1980, p. 1-23.

Burnett Charles, «A Group of Arabic-Latin Translators Working in Northern Spain in the Mid-12th Century », Journal of the Royal Asiatic Society of Great Britain and Ireland, 1977, p. 62-108.

Burnett, "Aristotle as an Authority on Judicial Astrology », in Meirinhos José Francisco, Weijers Olga (éd.), Florilegium mediaevale. Etudes offertes à Jacqueline Hamesse à l'occasion de son éméritat, Louvain-la-Neuve, 2009, p. 39-62.

Burnett, « King Ptolemy and Alchandreus the Philosopher : The Earliest Texts on the Astrolabe and Arabic Astrology at Fleury, Micy and Chartres », Annals of Science, 55, 1998, p. 329-368.

Burnett, «Late Antique and Medieval Latin Translations of Greek Texts on Astrology and Magic », in The Occult Sciences in Byzantium, Magdalino Paul, Mavroudi Maria (éd.), Genève, 2006, p. 325-359.

Burnett, «'Partim de suo et partim de alieno' : Bartholomew of Parma, the Astrological Texts in MS Bernkastel-Kues, Hospitalsbibliothek 209, and Michael Scot », in Battistini Pierluigi (éd.), Seventh Centenary of the Teaching of Astronomy in Bologna, 1297-1997. Actes du colloque de Bologne (1997), Bologna, 2001, p. 37-76.

Burnett, « Ten or forty? A Confusing Numerical Symbol in the Middle Ages » in Mathematics Celestial and Terrestrial. Festschrift für Menso Folkerts zum 65. Geburtstag, p. 81-90.

Burnett, «The Planets and the Development of the Embryo ", in The Human Embryo. Aristotle and the Arabic and European Traditions, Dunstan Gordon R. (éd.), Exeter, 1990.

Burnett, « An Unknown Latin Version of an Ancient Parapegma: The Weather-Forecasting Stars in the Iudicia of Pseudo-Ptolemy, in Making Instruments Count: Essays on Historical Scientific Instruments presented to Gerard L'Estrange Turner, R.G.W. Anderson et al. (éd.), Aldershot, 1993, p. 27-41.

Burnett, « Vincent of Beauvais, Michael Scot and the 'New Aristotle' ", in Vincent de Beauvais. Lector et compilator, Paulmier-Foucart Monique, Lusignan Serge (éd.), Grâne, 1997, p. 189-213.

Caiazzo Irene, Burnett Charles, « New Manuscripts of On the Astrolabe by Raymond of Marseille », Scriptorium, 65, 2011, p. 338-349.

Callus Daniel A., " Philip the Chancellor and the De Anima ascribed to Robert Grosseteste ", Mediaeval and Renaissance Studies, 1, 1941/ 1943, p. 105-127. 
Callus, « The Powers of the Soul. An Early Unpublished Text », Recherches de théologie ancienne et médiévale, 19, 1952.

Cano Gonzàlez Ana Maria, Germain Jean, Kremer Dieter, Dictionnaire historique de l'anthroponymie romane. Vol. II/ 1 : L'homme et les parties du corps humain, Tübingen, 2004.

Carmody Francis, Arabic Astronomical and Astrological Sciences in Latin Translation. A Critical Bibliography, Berkeley-Los Angeles, 1956.

Catalogus codicum manuscriptorum medii aevi latinorum qui in bibliotheca Jagellonica Cracoviae asservantur, 1980, vol. I.

Chantilly. Le Cabinet des livres manuscrits, t. I, Théologie - Jurisprudence - Sciences et arts, Paris, 1900.

Crombie Alistair C., Robert Grosseteste and the Origins of Experimental Science (1100-1700), Oxford, $1961^{2}$.

Cullen Christopher M., « Alexander of Hales », in Lagerlund Henrik (éd.), Encyclopedia of Medieval Philosophy: Philosophy between 500 and 1500, Dordrecht, 2011, p. 62-64.

d'Alverny Marie-Thérèse, Alain de Lille. Textes inédits avec une introduction sur sa vie et ses œuvres, Paris, 1965.

Dales Richard C., « Robert Grosseteste’s Scientific Works », in Isis, 52/3, 1961, p. 381-402.

Dales, « The Influence of Grosseteste's Hexaemeron on the Sentences Commentaries of Richard Fishacre OP and Richard Rufus of Cornwall OFM », Viator, 2, 1971, p. 271-300.

de Callataÿ Godefroid, Annus platonicus, A Study of World Cycles in Greek, Latin and Arabic Sources, Louvain-la-Neuve, 1996 (Publications de l'Institut Orientaliste de Louvain, 47).

de Miramon Charles, «Spiritualia et Temporalia - Naissance d'un couple », Zeitschrift der SavignyStiftung für Rechtsgeschichte. Kanonistische Abteilung, 123/92, 2006, p. 224-287.

Deus Janine, Der 'Experimentator' - Eine anonyme lateinische Naturenzyklopädie des frühen 13. Jahrhunderts, Diss. Geschichtswissenschaft, Hamburg, 1998.

Dinkova-Bruun, Greta, et al. (éd.), The Dimensions of Colour : Robert Grosseteste's De colore, Toronto, 2013 (Durham Medieval and Renaissance Texts, 4).

Distelbrink Balduinus, Bonaventurae scripta authentica, dubia vel spuria critice recensita, Roma, 1975.

Donati Silvia, « Un nuovo testimone dello Scriptum super Metaphysicam di Riccardo Rufo di Cornwall (Salamanca, Bibl. Univ., Ms. 2322) », Bulletin de philosophie médiévale, 45, 2003, p. 31-60.

Draelants Isabelle, « Le dossier des livres sur les animaux et les plantes de Iorach : tradition occidentale et orientale », in Draelants Isabelle, Tihon Anne, Van den Abeele Baudouin (éd.), Occident et Proche-Orient : contacts scientifiques au temps des croisades. Actes du colloque de Louvain-laNeuve, 24-25 mars 1997, Louvain-la-Neuve, Turnhout, 2000 (Réminisciences), p. 191-276.

Draelants, Un encyclopédiste méconnu du XIII ${ }^{e}$ siècle : Arnold de Saxe. Cuvres, sources, réception, Louvain-la-Neuve, 2000 (thèse d'histoire).

Draelants, « La science encyclopédique des pierres au $13^{\mathrm{e}}$ siècle : l'apogée d'une veine minéralogique », in Thomasset Claude, Ducos Joëlle, Chambon Jean-Pierre (dir.), Aux origines de la géologie de l'Antiquité au Moyen Âge. Actes du colloque international 10-12 mars 2005, Paris Sorbonne (Paris IV), Paris, 2010, p. 91-139.

Draelants, «La science naturelle et ses sources chez Barthélemy l'Anglais et les encyclopédistes contemporains ", in Van den Abeele, Meyer 2005, p. 43-99. 
Draelants, « Le De generatione et corruptione au 'siècle d'or' des encyclopédies médiévales », in Ducos Joëlle, Giacomotto-Charra Violaine (éd.), Lire Aristote au Moyen Age et à la Renaissance. Réception du traité Sur la génération et la corruption, Paris, 2011.

Draelants, «Le De iudiciis virtutum et viciorum d'Arnold de Saxe, un florilège moral sous forme de disputatio [avec édition critique] », in Ceulemans R., De Leemans P. (éd.), Good Authority. Tradition, Compilation and the Construction of Authority in Literature from Antiquity to the Renaissance, Turnhout, 2015 (LECTIO Studies on the Transmission of Texts and Ideas, 3), p. 299-451.

Draelants Isabelle, Frunzeanu Eduard, « Sur les traces du De motibus / iudiciis planetarum attribué à Ptolémée », Early Science and Medicine, 16, 2011, p. 571-599.

Draelants Isabelle, Paulmier-Foucart Monique, «Echanges dans la societas des naturalistes au milieu du XIII ${ }^{\mathrm{e}}$ siècle : Arnold de Saxe, Vincent de Beauvais et Albert le Grand », in D James-Raoul Danièle, Soutet Olivier, (dir.), Par les mots et les textes..., Mélanges de langue, de littérature et d'histoire des sciences médiévales offerts à Claude Thomasset, Paris, 2005, p. 219-238.

Eastwood Bruce S., Ordering the Heavens. Roman Astronomy and Cosmology in the Carolingian Renaissance, Leiden/ Boston, 2007.

Épiney-Burgard Georgette, Gérard Grote, fondateur de la Dévotion Moderne : Lettres et traités, Turnhout, 1998.

Flood John, Ginther James R., Goering Joseph W. (éd.), Robert Grosseteste and His Intellectual Milieu. New Editions and Studies, Toronto, 2013 (Pontifical Institute of Mediaeval Studies, 24).

French Roger, « Astrology in Medical Practice », in Garcia-Ballester Luis, French Roger, Arrizabalaga Jon, Cunningham Andrew (éd.), Practical Medicine from Salerno to the Black Death, Cambridge, 1994, p. 30-59.

French, « Foretelling the Future: Arabic Astrology and English Medicine in the Late Twelfth Century », Isis, 87/3, 1996, p. 453-480.

Fried Johannes, Les fruits de l'Apocalypse. Origines de la pensée scientifique moderne au Moyen Âge, Paris, 2004.

Frunzeanu Eduard, recension de : «Barthélemy l'Anglais, De proprietatibus rerum, t. 1, introd. B. Van den Abeele, Prohemium, éd. H. Meyer, Liv. I, éd. M. W. Twomey, Liv. II, éd. B. Roling, Liv. III et IV, éd. R. James Long ; t. 6, Liv. XVII, éd. I. Ventura, Turnhout, Brepols, 2007 », Le Moyen Âge, 3-4, 2009, p. 617-621.

Galle Griet, « The Dating and Earliest Reception of the translatio vetus of Aristotle's De sensu », Medioevo. Rivista di storia della filosofia medievale, 33, 2008, p. 7-96.

Gauthier René-Antoine, « Notes sur les débuts (1225-1240) du premier 'averroïsme' », Revue de sciences philosophiques et théologiques, 66, 1982, p. 321-374.

Goering Joseph W., « When and Where did Grosseteste Study Theology? » in McEvoy James (éd.), Robert Grosseteste : New Perspectives on his Thought and Scholarship, Turnhout, 1995 (Instrumenta Patristica, 27), p. 17-51.

Grant Edward, Planets, Stars, and Orbs. The Medieval Cosmos, 1200-1687, Cambridge, 1996.

Henquinet Francois-Marie, « Un recueil de questions annoté par S. Bonaventure », Archivum Franciscanum historicum, 25, 1932, p. 553-555.

Hübner Wolfgang, « Astrologie et mythologie dans la Tétrabible de Ptolémée d'Alexandrie », in Sciences exactes et sciences appliquées à Alexandrie (III ${ }^{e}$ siècle av. J.-C. - I Ir siècle ap. J.-C.), p. 325-345. 
Juste David, Les manuscrits astrologiques latins conservés à la Bayerische Staatsbibliothek de Munich, Paris, 2011 (Catalogus Codicum Astrologorum Latinorum, I).

Ker Neil R., Piper A. J., Medieval Manuscripts in British Libraries, Oxford, 1992, vol. IV.

Kibre Pearl, « 'Astronomia' or 'astrologia' Ypocratis », Studies in Mediaeval Science, Alchemy, Astrology, 1984, p. 133-156.

King Donald A., Astrolabes from Medieval Europe, Farnham, 2011.

Kristeller Paul O., Iter italicum, London, Leiden, 1967.

Kunitzsch Paul, Typen von Sternverzeichnissen in astronomischen Handschriften des zehnten bis vierzehnten Jahrhunderts, Wiesbaden, 1966.

Kunitzsch Paul, «Abū Ma‘šar, Johannes Hispalensis und Alkameluz », Zeitschrift der Deutschen Morgenländischen Gesellschaft, 120, 1970, p. 103-125 (repr. in The Arabs and the Stars, art. XVII).

Kunitzsch Paul, «Zur Tradition der 'Unwettersterne' », Zeitschrift der Deutschen Morgenländischen Gesellschaft, 122, 1972, p. 108-117 (repr. in The Arabs and the Stars, art. XVI).

Kunitzsch Paul, The Arabs and the Stars. Texts and Traditions on the Fixed Stars, and their Influence in Medieval Europe, Northampton, 1989 (Variorum Reprints).

Kunitzsch Paul, «Zur Problematik der Astrolabsterne : eine weitere unbrauchbare Sterntafel », Archives internationales d'histoire des sciences, 43, 1993, p. 197-208.

Kunitzsch Paul, Dekker Elly, "The Stars on the Rete of the so-called 'Carolingian Astrolabe' », in Casulleras Josep, Samsó Julio (éd.), From Baghdad to Barcelona. Studies in the Islamic Exact Sciences in Honour of Prof. Juan Vernet, Barcelona, 1996, p. 655-672 (repr. in Stars and Numbers, art. XVIII).

Lafleur Claude, «Transformations et permanences dans le programme des études à la Faculté des arts de l'Université de Paris au XIII ${ }^{\mathrm{e}}$ siècle : Le témoignage des 'introductions à la philosophie' et des 'guides de l'étudiant' ", Laval théologique et philosophique, 54, 1998, p. 387-410.

Le Bœuffle André, Les Noms latins d'astres et de constellations, Paris, $2010^{2}$.

Lehmann Paul, Mittelalterliche Handschriften des K. B. Nationalmuseums zu München, München, 1916, (Sitzungsberichte der Königl. Bayerischen Akademie der Wissenschaften, philos.-philol. und hist. Klasse, Jg. 1916, 4. Abhandlung).

Lehoux Daryn, Astronomy, Weather, and Calendars in the Ancient World, Cambridge-New York, 2007.

Lemay Helen R., « Science and Theology at Chartres : The Case of the Supracelestial Waters », in The British Journal for the History of Science, 10, 1977, p. 226-236.

Llinarès Armand, « Le lullisme de Lefèvre d'Etaples et de ses amis humanistes ", in L'humanisme français au début de la Renaissance, Paris, 1973, p. 127-135.

Lloyd Geoffrey E. R., Une histoire de la science grecque, Paris, 1993.

Long Raymund J., Noone Timothy B., " Fishacre and Rufus on the Metaphysics of Light : two Unedited Texts ", in Hamesse Jacqueline (éd.), Roma, magistra mundi. Itineraria culturae medievalis. Mélanges offerts au Père L. E. Boyle à l'occasion de son $75^{e}$ anniversaire, Louvain-la-Neuve, 1998, p. 517-548.

Long Raymund J., « The Anonymous De anima of Assisi, Biblioteca Comunale ms. 138 », in Universalità della Ragione. Pluralità delle Filosofie nel Medioevo XII Congresso Internazionale di Filosofia Medievale de la Société Internationale pour l'Étude de la Philosophie Médiévale (S.I.E.P.M.) Palermo, 17-22 settembre 2007, Musco A., D’Agostino S., Musotto G., Palmeri P., Spallino P. (éd.), Palermo, 2007, p. 271-280. 
Long Raymund J.« The Division of the Waters (Gen 1,6-7) : the History of a Conundrum and its Resolution by the Early Oxford Masters ", in Emery Kent, Friedman Russell L., Speer Andreas (éd.), Philosophy and Theology in the Long Middle Ages. A Tribute to Stephen F. Brown, Leiden, Boston, 2011.

Lucentini Paolo, « L'Asclepius ermetico nel secolo XII », in Westra Haijo Jan, Jeauneau Édouard (éd.), From Athens to Chartres: Neoplatonism and Medieval Thought : Studies in Honour of Edouard Jeauneau..., Leiden, 1947, p. 397-420.

Mac Niocaill Gearoid, «Bartholomaei Anglici de proprietatibus rerum liber octavus. Leagan Gaeilge o thus na 15u aoise », Celtica, 8, 1968, p. 201-242; 9, 1971, p. 266-314.

Malewicz Malgorzata Hanna, « Le Libellus de efficatia artis astrologice : Traité astrologique d'Eudes de Champagne, XII ${ }^{\mathrm{e}}$ siècle ", Mediaevalia Philosophica Polonorum, 20, 1974, p. 3-95.

McEvoy James, The Philosophy of Robert Grosseteste, Oxford, $1986^{2}$.

Meier Christel E., (éd.), Die Enzyklopädie im Wandel vom Hochmittelalter bis zur frühen Neuzeit, München, 2002 (Münstersche Mittelatler-Schriften, 78).

Meyer Heinz, « Die illustrierten lateinischen Handschriften im Rahmen der Gesamtüberlieferung der Enzyklopädie des Bartholomäus Anglicus ", Frühmittelalterliche Studien, 30, 1996, p. 368-395.

Meyer Heinz, Die Enzyklopädie des Bartholomäus Anglicus. Untersuchungen zur Überlieferungs- und Rezeptionsgeschichte von De proprietatibus rerum, München, 2000 (Münstersche MittelalterSchriften, 77).

Muccillo Maria, « Der ,scholastische' Hermetismus des Annibale Rosselli und die Trinitatslehre », in Mulsow Martin (éd.), Das Ende des Hermetismus, Tübingen, 2002, p. 61-101.

North John D., Chaucer's Universe, Oxford, 1988.

Panti Cecilia, Moti, virtù e motori celesti nella cosmologia di Roberto Grossatesta. Studio ed edizione dei trattati De sphera, De cometis, De motu supercelestium, Firenze, 2001 (Testi e studi per il Corpus Philosophorum Medii Aevi, 16).

Paulmier-Foucart Monique, « Le plan et l'évolution du Speculum maius de Vincent de Beauvais : de la version bifaria à la version trifaria », in Meier, Die Enzyklopädie im Wandel, p. 245-268.

F. S. Pedersen, The Toledan Tables, København, 2002.

Pellegrin Elisabeth, Bouhot Jean-Paul, Catalogue des manuscrits médiévaux de la Bibliothèque municipale d'Orléans, Paris, 2010.

Pelster Franz, «Quästionen des Franziskaners Richardus Rufus de Cornubia (um 1250) in Cod. VII C. 19 der Nationalbibliothek Neapel und Cod. 138 der Stadtbibliothek Assisi », Scholastik, 14, 1939, p. 215-233.

Pereira Michela, « Bernardo Lavinheta e la diffusione del lullismo a Parigi nei primi anni del '500 », Interpres. Rivista di studi quattrocenteschi, 5, 1983/1984, p. 242-265.

Pingree David E., «The Diffusion of Arabic Magical Texts in Western Europe », in La diffusione delle scienze islamiche nel Medio Evo Europeo, Scarcia Amoretti Biancamaria (éd.), Rome, 1987, p. 57-102.

Poulle Emmanuel, «Quand le soleil a rendez-vous avec la lune », Il sole e la luna. The Sun and the Moon (Micrologus. Natura, Scienze e Società Medievali), XII, 2004.

Raedts Peter, Richard Rufus of Cornwall and the Tradition of Oxford Theology, Oxford, 1987, p. 64-114. 
Rigo Caterina, « Zur Redaktionsfrage der Frühschriften des Albertus Magnus », in Honnefelder Ludger (dir.), Albertus Magnus und die Anfänge der Aristoteles-Rezeption im lateinischen Mittelalter, Münster, 2005, p. 325-374.

Rouse Richard H., Rouse Mary A., « The Verbal Concordance to the Scriptures ", Archivum fratrum praedicatorum, 44, 1974, p. 5-30.

Saenger Paul, «The British Isles and the Origin of the Modern Mode of Biblical Citation », Syntagma. Revista del Instituto de Historia del Libro y de la Lectura, 1, 2005, p. 77-115.

Schipke Renate, Die mittelalterlichen Handschriften der Ratsschulbibliothek Zwickau, Berlin, 1990.

Sezgin Faut, Geschichte des arabischen Schrifttums, t. VII. Astrologie - Meteorologie und Verwandtes bis ca. 430 H, Leiden, 1979.

Seymour Michael C., Bartholomeus Anglicus and his encyclopedia, Aldershot, 1992.

Smith, Mark A., Alhacen's Theory of Visual Perception, Philadelphia, 2 vol., 2001.

Somfai Anna, « The Eleventh-Century Shift in the Reception of Plato's 'Timaeus' and Calcidius' 'Commentary' ", Journal of the Warburg and Courtauld Institutes, 65, 2002, p. 1-21.

Sturlese Loris, «Florilegi filosofici ed enciclopedie in Germania nelle prima metà del duecento. Gli scritti di Arnoldo di Sassonia e di Bartolomeo l'Inglese e la diffusione della scienza araba e aristotelica nella cultura tedesca », Giornale critico della filosofia italiana, 69 (81), fasc. 3, 1990, p. 293-318.

Thomson Samuel H., « The Text of Grosseteste's De cometis », Isis, 19, 1933, p. 19-25.

Thomson Samuel H., The Writings of Robert Grosseteste Bishop of Lincoln, 1235-1253, Cambridge, 1940.

Thorndike Lynn, A History of Magic and Experimental Science, New York, 1923.

Thorndike Lynn, « Notes upon some Medieval Astronomical, Astrological and Mathematical Manuscripts at Florence, Milan, Bologna and Venice », Isis, 50, 1959, p. 33-50.

Thorndike Lynn, « The Three Latin Translations of the Pseudo-Hippocratic Tract on Astrological Medicine ", Janus. Revue internationale de l'histoire des sciences, de la médecine, de la pharmacie et de la technique, 49, 1960, p. 104-129.

Truhlár̆, Josf, Catalogus codicum manu scriptorum latinorum qui in C. R. Bibliotheca publica atque Universitatis Pragensis asservantur, Prague, 1905.

Valentinelli Joseph, Bibliotheca manuscripta ad S. Marci Venetiarum, Venezia, 1868.

Van den Abeele Baudouin, « Moralisierte Enzyklopädien in der Nachfolge von Bartholomäus Anglicus : das 'Multifarium' in Wolfenbüttel und der 'Liber de exemplis et similitudinibus rerum' des Johannes de Sancto Geminiano », in Meier, Die Enzyklopädie im Wandel, p. 279-304.

Van den Abeele Baudouin, «A la recherche de l'Experimentator de Thomas de Cantimpré », in Bénatouill Thomas, Draelants Isabelle (éd.), Expertus sum. L'expérience par les sens dans la philosophie naturelle médiévale. Actes du colloque international de Pont-à-Mousson, 5-7 février 2009, Firenze, 2011 (Micrologus' Library, 40).

Van den Abeele Baudouin, Meyer Heinz (éd.) Bartholomaeus Anglicus, De proprietatibus rerum. Texte latin et réception vernaculaire. Lateinischer Text und volkssprachige Rezeption, Turnhout, 2005 (De diversis artibus, 74, N.S., 37), p. 43-99.

Vanhamel Willy, « Biobibliographie de Guilla 


\section{NOTES}

1. Le commentaire des sources du livre VIII du De proprietatibus rerum a été rédigé initialement par I. Draelants et E. Frunzeanu pour accompagner l'édition critique (établie principalement par Iolanda Ventura, avec notre collaboration), remise à l'éditeur en 2012. Suite aux retards considérables pris par la publication, nous avons décidé de publier séparément ce commentaire.

Pour la caractérisation de certaines œuvres contenues dans les manuscrits astronomiques, le présent commentaire a bénéficié des remarques de Charles Burnett et de David Juste, qu'il est un plaisir de remercier.

2. C'est pourquoi nous avons choisi de retenir ce titre au livre VIII.

3. BaRtholomaeus ANGlicus, De proprietatibus rerum VIII, c.1, 1.2-26: Mundus itaque, ut dicit Mercurius, dicitur tribus modis. Nam mundus dicitur divinus intellectus, mundus scilicet archetipus, incorporeus, invisibilis et eternus, ad cuius exemplar mundus sensibilis est creatus [...] Secundo modo dicitur mundus universitas eorum que celi ambitu continentur, ut celum, in quo sidera lucent, ignis, quo omnia calent, aer, quo omnia viventia spirant et vigent, aqua, que terre latera circumcingit, terra, que omnia inferiora sustinet atque nutrit, de quo dicitur 'Mundus per ipsum factus est'. Tertio dicitur homo minor mundus, quia totius mundi imaginem in se representat. Est itaque primus mundus eternus in divina mente eternaliter permanens. Secundus mundus perpetuus est, divina voluntate esse perpetuum et originem ex nihilo contrahens. Tertius secundum quid est perpetuus, secundum quid est caducus, omnium similitudinem in se gerens. De primo autem mundo et tertio in precedentibus dictum est. Nunc de mundo sensibili aliquid dicendum est.

4. En commentant le Timée de Platon, Guillaume de Conches propose une dichotomie semblable (I, c. 43, éd. Jeauneau $2006: 78,1.18-23$ ) : Quod ut melius intelligatur, quid proprie sit aeternum, quid sempiternum, quid perpetuum dicamus. Et est aeternum quod caret principio et fine ut Creator; sempiternum quod, carens fine, habet principium non in tempore sed cum tempore ut mundus; perpetuum est quod incipit in tempore et caret fine ut anima hominis.

5. Analyse de ce passage par P. LUCENTINI, « L'Asclepius ermetico nel secolo XII », in WESTRA H. J. (éd.), From Athens to Chartres: Neoplatonism and Medieval Thought: Studies in Honour of Edouard Jeauneau..., Leiden, 1947, p. 397-420 : 416-417.

6. DANIEL DE MORLEY, Philosophia mundi, éd. SUDHOFF $1917: 8$.

7. PAUL, Ep. ad Rom., I, c. 20 : Invisibilia Dei a creatura mundi, per ea quae facta sunt, intellecta conspiciuntur ; AUGUSTIN, De doctrina christiana, II, c. 16, 24, éd. MARTIN 1962 : 49-50, sur la nécessité de connaître les propriétés des choses : Rerum autem ignorantia facit obscuras figuratas locutiones, cum ignoramus uel animantium uel lapidum uel herbarum naturas aliarumue rerum, quae plerumque in scripturis similitudinis alicuius gratia ponuntur; (...) nam et de serpente (...), quantum inlustrat sensum illum, quod dominus iubet astutos nos esse 'sicut serpentes', ut scilicet (...) Ut ergo notitia naturae serpentis inlustrat multas similitudines, quas de hoc animante scriptura dare consueuit, sic ignorantia nonnullorum animalium, quae non minus per similitudines commemorat, impedit plurimum intellectorem. Sic lapidum, sic herbarum, uel quaeque tenentur radicibus. Nam et carbunculi notitia, quod lucet in tenebris, multa inluminat etiam obscura librorum, ubicumque propter similitudinem ponitur, et ignorantia berylli uel adamantis claudit plerumque intellegentiae fores.

8. DPR VIII, Prologus, 1. 2-15: Postquam, auxiliante Deo, tractatum de divinis nominibus, de angelorum proprietatibus nec non de homine et eius partibus et accidentalibus ipsius conditionibus complevimus, restat ut ad proprietates mundi sensibilis, quantum nobis dabitur, desuper manus apponamus, ut materiam divine laudis ex proprietatibus operum possimus elicere Conditoris. Invisibilia enim Dei per ea que facta sunt intellecta conspiciuntur, ut dicit Apostolus. Et ideo aliquas mundi huius et contentorum ipsius proprietates huic opusculo sub brevi compendio interserere proponimus, ut per similitudinem 
proprietatum corporalium et intellectum spiritualem et misticum facilius in divinis Scripturis intelligere valeamus. A proprietatibus itaque mundi primitus inchoandum est.

9. DPR VIII.17, 1. 36-39 et VIII.18, 1. 5-7 : Unde Ambrosius in Hexameron sic nobis describit virtutes ipsius Solis dicens: Sol est oculus mundi, iocunditas diei, pulcritudo celi, mensura temporum, virtus et vigor omnium nascentium, dominus planetarum, decor et perfectio omnium stellarum ; Luna enim, ut dicitur in Hexameron, est decor noctis, mater roris, humoris ministra, dominatrix maris, temporum mensura, emulatrix Solis, immutatrix aeris.

10. Nous appelons «marqueur de source » la référence médiévale (le titulus) à un auteur et/ou à une œuvre, placée par le compilateur avant une ou plusieurs citations.

11. C. LAFLEUR, «Transformations et permanences dans le programme des études à la Faculté des arts de l'Université de Paris au XIII siècle : Le témoignage des 'introductions à la philosophie' et des 'guides de l'étudiant' ", Laval théologique et philosophique, 54, 1998, p. 387-410: 406.

12. AL- BATTĀNĪ, Albatenii Opus astronomicum... éd. NALLINO 1899-1907. Traduit à deux reprises, par Robert de Chester et par Platon de Tivoli, le traité est connu aussi sous d'autres titres : De scientia stellarum, De motu stellarum, De numeris stellarum.

13. De motibus astrorum, pourtant traduit dès 1217 par Michel Scot.

14. Traduit en latin à deux reprises, par Jean Hispalensis et Limiensis (Differentie scientie astrorum, éd. CARMODY 1943) et par Gérard de Crémone (Il 'Libro dell'aggregazione delle stelle' secondo il codice mediceo-laurenziano Pl. 29. cod. 9, CAMPANI R. (éd.), Città di Castello, 1910).

15. AL-BITRŪJī, De motibus celorum, éd. CARMODY 1952 : 35. F. J. Carmody passe rapidement en revue les auteurs médiévaux qui se sont intéressés au traité d'al-Bitrūjī (34-38).

16. C. PANTI, Moti, virtù e motori celesti nella cosmologia di Roberto Grossatesta. Studio ed edizione dei trattati De sphera, De cometis, De motu supercelestium, Firenze, 2001 (Testi e studi per il Corpus philosophorum Medii Aevi, 16), notamment 79-82 et 169-186.

17. Dès la rédaction de son De IV coaequaevis (1240-1250), Albert le Grand fait quelques références aux deux astronomes arabes (ALBERTUS MAGNUS, Summa de creaturis, éd. BORGNET 1895, t. 34 - qui édite la deuxième version du De IV coaequaevis -, pars I, tract. III, q. $8: 409,411$; tract. III, q. 12 : 426-427 ; tract. III, q. $13: 429$; tract. III, q. $16: 438$ ). Sur les versions de ce texte, voir l'étude de C. RIGO, «Zur Redaktionsfrage der Frühschriften des Albertus Magnus », in HONNEFELDER L. (éd.), Albertus Magnus und die Anfänge der Aristoteles-Rezeption im lateinischen Mittelalter, Münster, 2005, p. 325-374.

18. DPR VIII.1, 1. 63-84 : Unde istam materiam primam Plato in Tymeo, prout potuit, sic descripsit: Fuit, inquit, yle, id est materia prima, sine qualitate, sine quantitate, sine colore, sine specie, sine loco, sine tempore, inter aliquam substantiam et nullam et cetera. Hec verba multum sunt difficilia, tamen sic exponuntur, quia dicitur fuisse sine quantitate, non quia fuerit sine omni quanto, sed quia non fuit determinate quantitatis quo ad nos, sicut gigas dicitur immensus, quia excedit aliorum hominum quantitatem. Ideo etiam sine qualitate dicitur, quia non habuit a qualitate aliqua specialiter nominari. Non enim plus potuit calida quam frigida dici nec econverso, et sic de aliis. Sine colore dicitur, quia non habuit aliquem colorem innatum ab aliquo elemento. Sine tempore fuit, quia nondum fuerant tempora et temporum vicissitudines, quando hec materia est creata. Sine loco etiam fuit, quia non habuit determinatum situm plus sursum quam deorsum, nec plus in latum quam in longum. Inter aliquam substantiam est et nullam, quia nulla substantia materialis precessit illam, sed aliqua est secuta. A. SOMFAI , «The Eleventh-Century Shift in the Reception of Plato's 'Timaeus' and Calcidius' 'Commentary' ", Journal of the Warburg and Courtauld Institutes, 65, 2002, p. 1-21. Dans le traité De commixtionibus elementorum, Urso de Salerne discute des attributs de la matière à partir de la même citation du Timée.

19. Les sondages que nous avons faits dans les manuscrits du corpus dionysien de l'Université de Paris ou encore dans les commentaires sur les traités de Pseudo-Denys l'Aréopagite (Guillaume de Lucques, Thomas Gallus, Albert le Grand, Robert Grosseteste) n'ont pas été fructueux. 
20. Das Decretum gelasianum de libris recipiendis et non recipiendis, éd. voN DOBSCHÜTZ 1912 : 36-37 et 44-45.

21. Dans la traduction française médiévale de Jean Corbechon, c'est le nom de Rabbi Moise (Maïmonide) qui figure à la place de Rabanus.

22. DPR VIII.2, 1. 90-94: Unde axem vocat Aristoteles quamdam lineam intellectualem porrectam a polo usque ad polum diametraliter et extensam, circa cuius medium totius celi ambitus equaliter circumfertur, sicut dicit ibidem Commentator.

23. R.-A. GAUTHIER, "Notes sur les débuts (1225-1240) du premier 'averroïsme' ", Revue de sciences philosophiques et théologiques, 66, 1982, p. 321-374, en part. 337-40.

24. J. DEUS, Der 'Experimentator' - Eine anonyme lateinische Naturenzyklopädie des frühen 13. Jahrhunderts, Diss. Geschichtswissenschaft, Hamburg, 1998.

25. Berlin, Deutsche Staatsbibliothek, Hamilton 277 ; Pisa, Bibl. Cateriniana, 30 ; Roma, Bibl. Angelica, 1038 (R.7.1) ; Stuttgart, Württembergische Landesbibliothek, med. et phys. $2^{\circ} 24$.

26. Arras, Bibl. municipale 76. ; Chambéry, Bibl. municipale, 30 ; London, British Library, Sloane 3167 ; Madrid, Bibl. Nacional, 12803 ; Milan, Bibl. Ambrosiana, A 147 sup.; Soissons, Bibl. municipale, 26.

27. Wolfenbüttel, Herzog August Bibliothek, 8.8 Aug. $4^{\circ}$.

28. H. MEYER, Die Enzyklopädie des Bartholomäus Anglicus. Untersuchungen zur Überlieferungs- und Rezeptionsgeschichte von De proprietatibus rerum, München, 2000 (Münstersche MittelalterSchriften, 77) : 149-163, qui ne fait pourtant pas référence à la thèse de J. Deus. Par ailleurs, aux manuscrits retenus par J. Deus, H. Meyer et B. Van den Abeele ont ajouté d'autres témoins, sans les rapprocher de l'une ou l'autre version de l'Experimentator: Cambridge (MA), Harvard College Library, Riant 89 ; Cortona, Biblioteca communale, 85 ; Firenze, Bibl. naz. Centrale, Landau Finaly 58 ; Leiden, Bibl. Rijksuniversiteit, Voss. Lat. Q 50 ; Pavia, Bibl. Universitaria, Aldini 165 ; Città del Vaticano, Bibl. Apost. Vat., Chigi E.V.160. Un autre manuscrit décrit dans le catalogue de l'antiquaire Jacques Rosenthal (catalogue 90, 1928, item 118) n'a pas été retrouvé.

29. B. VAN DEN ABEELE, "A la recherche de l'Experimentator de Thomas de Cantimpré», in BÉNATOUÏL T., DRAELANTS I. (éd.), Expertus sum. L'expérience par les sens dans la philosophie naturelle médiévale. Actes du colloque international de Pont-à-Mousson, 5-7 février 2009, Firenze, (Micrologus' Library, 40), 2011, p. 41-65.

30. Nous avons constaté que le texte de la Hierarchia repris par l'Experimentator est entremêlé de quelques notes sur les anges qui, dans les manuscrits, se trouvent à la fin du traité d'Alain de Lille. Marie-Thérèse d'Alverny signale la présence de ces notes dans trois des cinq manuscrits qui conservent le texte, mais choisit de ne pas les éditer (Alain de Lille. Textes inédits avec une introduction sur sa vie et ses cuvres, Paris, 1965, p. 219-235). Cela signifie que l'Experimentator de la rédaction I transmise dans le manuscrit de Stuttgart a dû avoir accès à une famille particulière des manuscrits de la Hierarchia d'Alain de Lille.

31. Pour le texte de l'Experimentator, nous renvoyons à l'édition DEus 1998 pour les citations communes aux trois versions, et aux manuscrits de Chambéry $(\mathrm{Ch})$ et de Stuttgart (St) dans le reste des cas ; Remigii Autissiodorensis Commentum in Martianum Capellam, éd. LUTZ 1963 et 1965; Meteorologica, éd. SCHOONHEIM 2000 ; ROBERT GROSSETESTE, Hexaëmeron, éd. DALES, GIEBEN 1982.

32. DPR VIII.2, 1. 154-156: Unde motus celi est totius temporis primum subiectum, et modus et regula omnium motuum aliorum.

33. Experimentator (Stuttgart, Württembergische Landesbibliothek, med. et phys. $2^{\circ} 24, \mathrm{f} .93 \mathrm{v}$, Berlin, Deutsche Staatsbibliothek, Hamilton 277, f. 48ra); ROBERT GROSSETESTE, Hexaëmeron, I, 10-11: 99. Nous n'avons pas retrouvé cette phrase chez Barthélemy l'Anglais.

34. Voir les introductions aux éditions du commentaire de Rémi d'Auxerre : éd. LUTZ 1963 et I. RAMELLI, Tutti i commenti a Marziano Capella, Milano, 2006. 
35. DPR VIII.10, 1. 4-8 : Aries ... quia sicut Aries in iacendo super latus unum equaliter mutat vices, sic Sol existens in illa parte zodiaci, que dicitur Aries, facit equinoctium et equales facit dies artificiales atque noctes.

36. Ed. partielle THORNDIKE 1949, appendix V :477-80.

37. On trouvera une brève description des œuvres d'Arnold de Saxe, de leurs rapports entre elles, de leur chronologie et de leur tradition manuscrite dans I. DRAELANTS, « Le De iudiciis virtutum et viciorum d'Arnold de Saxe, un florilège moral sous forme de disputatio [avec édition critique] », in Ceulemans R., De leemans P. (éd.), Good Authority. Tradition, Compilation and the Construction of Authority in Literature from Antiquity to the Renaissance, (LECTIO Studies on the Transmission of Texts and Ideas, 3), Turnhout, 2015, p. 299-451.

38. Sur ces emprunts et les sources communes entre Barthélemy et Arnold de Saxe, voir I. DRAELANTS, « La science naturelle et ses sources chez Barthélemy l'Anglais et les encyclopédistes contemporains ", in VAN DEN ABEELE B., MEYER H. (éd.), Bartholomaeus Anglicus, De proprietatibus rerum. Texte latin et réception vernaculaire. Lateinischer Text und volkssprachige Rezeption, Turnhout, 2005, p. 43-99, avec en annexe une édition synoptique de passages communs aux deux auteurs.

39. G. GALLE, "The Dating and Earliest Reception of the translatio vetus of Aristotle's De sensu ", Medioevo. Rivista di storia della filosofia medievale, 33, 2008, p. 7-96: 67.

40. I. DRAELANTS, E. FRUNZEANU, "Sur les traces du De motibus / iudiciis planetarum attribué à Ptolémée », Early Science and Medicine, 16, 2011, p. 571-599.

41. Sur la division du Zodiaque et le choix des termes Chelae ou Libra pour désigner le signe de la Balance, A. Le Beuffle, Les Noms latins d'astres et de constellations, Paris, 2010², p.169-173 ; B. BAKHOUCHE, Les textes latins d'astronomie : un maillon dans la chaîne du savoir, Leuven, 1996 : 132-133.

42. PSEUdo-PTOlomaeus, Proportiones, éd. in JUSTE, D., Les Alchandreana primitifs. Étude sur les plus anciens traités astrologiques latins d'origine arabe ( $X^{e}$ siècle), Leiden, 2007 (Brill's Studies in Intellectual History, 152) : 510, 1.13. L'auteur des Proportiones utilise l'archaïsme Chelae pour désigner le Scorpion, ce qui pourrait indiquer que les Iudicia de Pseudo-Ptolémée se sont fondés sur un texte proche des Proportiones.

43. DPR VIII.22, 1. 106-109: Item secundum Aristotelem in libro De celo et mundo stelle sunt ex materia corporis celi, in quo posite sunt atque fixe, et ideo naturaliter sunt splendide sicut et celum, in quo revolvuntur.

44. DPR VIII.2, 1. 215-218 : Unde Macrobius in libro Ciceronis: Impulsu et motu ipsorum orbium efficitur sonus ille, qui acuta cum gravibus temperans equalem sonum efficit et concentum ; DPR VIII.6, 1. 61-63 : Unde Macrobius: Impulsu et motu ipsorum orbium efficitur sonus ille, et acuta cum gravibus temperans efficit concentus.

45. Arnold le déclare d'emblée dans le prologue à la première partie, intitulée De celo et mundo: Postquam in eius nomine completus est sermo de libris philosophorum, per ordinem textus sub eisdem verbis abreviatis a me, Arnoldo Saxone, numeroque centum et nonaginta, exceptis libris medicinalibus, ut facilius in componendis libris auctoritates sic paterent, nunc ergo [...].

46. Des citations du De animalibus de Iorach sont ainsi littéralement communes aux deux auteurs, cf. I. DRAELANTS, «Le dossier des livres sur les animaux et les plantes de Iorach: tradition occidentale et orientale ", in DRAELANTS I., TIHON A., VAN DEN ABEELE B. (éd.), Occident et ProcheOrient: Contacts scientifiques au temps des croisades. Actes du colloque de Louvain-la-Neuve, 24-25 mars 1997, Louvain-la-Neuve, Turnhout, 2000, p. 191-276 (une version plus complète des comparaisons de texte se trouve dans la thèse d'I. Draelants, Un encyclopédiste méconnu du XIII siècle: Arnold de Saxe. CEuvres, sources, réception, Louvain-la-Neuve, 2000 (thèse d'histoire), URL: http:// halshs.archives-ouvertes.fr/tel-00700745/). Pour une comparaison de l'usage que les deux auteurs ont fait du De generatione et corruptione, voir DRAELANTS, « Le De generatione et corruptione au 'siècle d'or' des encyclopédies médiévales ", in Ducos J., Giacomotto-charra V. (éd.), Lire Aristote 
au Moyen Age et à la Renaissance. Réception du traité Sur la génération et la corruption, Paris, 2011, p. 135-173.

47. En 1232 pour H. MEYER, «Die illustrierten lateinischen Handschriften im Rahmen der Gesamtüberlieferung der Enzyklopädie des Bartholomäus Anglicus », Frühmittelalterliche Studien, 30, 1996, p. 368-95: 368.

48. Sur les utilisations du De floribus rerum naturalium (en particulier le lapidaire) par Vincent de Beauvais et Albert le Grand, voir entre autres I. DRAELANTS - M. PAULMIER-FOUCART, «Échanges dans la societas des naturalistes au milieu du XIII ${ }^{\mathrm{e}}$ siècle : Arnold de Saxe, Vincent de Beauvais et Albert le Grand ", in James-Raoul D., Soutet O. (dir.), Par les mots et les textes..., Mélanges de langue, de littérature et d'histoire des sciences médiévales offerts à Claude Thomasset, Paris, 2005, p. 219-38, et DRAELANTS, «La science encyclopédique des pierres au $13^{\mathrm{e}}$ siècle: l'apogée d'une veine minéralogique ", in thomASSET C., DUCOS J., CHAMBON J.-P. (éd.), Aux origines de la géologie de l'Antiquité au Moyen Âge. Actes du colloque international 10-12 mars 2005, Paris Sorbonne (Paris IV), Paris, 2010, p. 91-139.

49. Magdebourg comme un des endroits d'activité d'Arnold de Saxe est l'hypothèse qui ressort des travaux d'I. DRAELANTS sur cet auteur. Le manuscrit d'Heidelberg, Universitätsbibl., Cod. Pal. germ. 263, f. 161r-171r donne pour auteur du De virtutibus gemmarum et lapidum (3 ${ }^{\mathrm{e}}$ partie du De floribus) Arnoldus Luce Magdeburgensis.

50. M.C. SEYMOUR, Bartholomeus Anglicus and his encyclopedia, Aldershot, 1992 : 1-4, s'est risqué à prolonger la biographie "saxonne" de Barthélemy jusqu'à une mort supposée en 1272, après avoir été le sixième ministre provincial de Saxe (puis d'Autriche) succédant à Conrad de Brunswic élu en 1247, et avoir exercé d'autres fonctions, plus hypothétiques, comme légat du pape Alexandre IV au nord des Carpathes et légat de Bohème, Moravie, Pologne et Autriche. L. STURLESE «Florilegi filosofici ed enciclopedie in Germania nelle prima metà del duecento. Gli scritti di Arnoldo di Sassonia e di Bartolomeo l'Inglese e la diffusione della scienza araba e aristotelica nella cultura tedesca ", Giornale critico della filosofia italiana, 69 (81), fasc. 3, 1990, p. 293-318, considère que l'achèvement du DPR a eu lieu en 1248.

51. Pour un accès en ligne à bon nombre d'œuvres de Grosseteste, cf. URL http:// www.grosseteste.com/download.htm.

52. Éd. DALES, GIEBEN, p. xii.

53. S.H. THOMSON, The Writings of Robert Grosseteste Bishop of Lincoln, 1235-1253, Cambridge, 1940 : 93 ; PANTI, Moti, virtù e motori celesti, 2001 : 360-364 ; Gr. Dinkova-Bruun et alii, (éd.), The Dimensions of Colour: Robert Grosseteste's De colore, Toronto, 2013 (Durham Medieval and Renaissance Texts, 4) : 74-78.

54. R. C. DALES, «Robert Grosseteste's Scientific Works », in Isis, 52/3, 1961, p. 381-402 : 402. Pour une table chronologique récente des écrits scientifiques de Robert Grosseteste: J. FLOoD, J.R. GINTHER, J. W. GOERING, Robert Grosseteste and His Intellectual Milieu. New Editions and Studies, Toronto, 2013 (Pontifical Institute of Mediaeval Studies, 24) : 185 ; contient aussi une édition critique du De luce par Cecilia Panti (193-237) et une traduction anglaise de cet opuscule par Neil Lewis (239-47). 55. Cf. J. MCEvoY, The Philosophy of Robert Grosseteste, Oxford, $1986^{2}, 3^{\mathrm{e}}$ partie, "The Light of Nature » : 149-222.

56. SAlimbene De AdAm, Cronica, éd. Scalia 1998-99 : 351-53 (Bari, 1966, t. I, 334-336) : Porro frater Hugo solitus erat dicere quod IIII habebat amicos quos specialiter diligebat [...] tertius fratris Hugonis amicus fuit Robertus Grossatesta Linconiensis episcopus, unus de maioribus clericis de mundo. Hic secundo post Burgundionem iudicem Pisanum transtulit Damascenum et Testamenta patriarcharum XII et multos alios libros. Quartus eius amicus fuit frater Adam de Marisco ex Ordine fratrum Minorum, unus de maioribus clericis de mundo. In Anglia claruit et multa scripta fecit sicut Linconiensis. Ambo fuerunt Anglici et ambo socii et ambo in episcopali ecclesia fuerunt sepulti. Tertius istorum duorum socius fuit magister Alexander ex Ordine fratrum Minorum, natione Anglicus et magister cathedratus Parisius, qui 
multa opera scripsit et, ut dicebant omnes qui eum bene noverant, suo tempore similem sibi in mundo non habuit. Le rappel de ce réseau d'amitiés est inséré par Salimbene sous l'année 1248, après avoir relaté un discours critiquant le népotisme des cardinaux tenu par Hugues de Digne, partisan du joachimisme, devant Innocent IV et le collège des cardinaux. On peut donc concevoir que la liste des amis est dressée avec le souci de créditer la personne d'Hugues de Digne et donc de donner du poids à ses paroles, et qu'elle n'apporte pas nécessairement des informations sur la décennie 1220-1230.

57. Cf. J. W. GOERING, «When and Where did Grosseteste Study Theology? » in MCEVoY J. (éd.), Robert Grosseteste: New Perspectives on his Thought and Scholarship, Turnhout, 1995 (Instrumenta Patristica, 27), p. 17-51 : 35-37. On connaît un document de legs d'une maison dans le cloître Saint-Opportune par un Robert Grosseteste marié et père de trois enfants. Le document est dressé par Guillaume d'Auvergne lui-même en février 1224. Il peut s'agir d'un parent très proche du savant Robert Grosseteste. Il ne faut toutefois pas négliger le fait que le nom Grosseteste apparaît dans plusieurs documents du XIII ${ }^{\mathrm{e}}$ siècle, en Angleterre comme en France et en Belgique, ce qui rend plausible l'existence de nombreux homonymes (v. la base de données Patronymica romanica, http://patrom.fltr.ucl.ac.be/, et les exemples réunis dans A. M. CANO GONZÀlEZ, J. GERMAIN, D. KREMER, Dictionnaire historique de l'anthroponymie romane. Volume II/1: L'homme et les parties du corps humain, Tübingen, 2004, sous les entrées caput : 155-156 et testa : 261-262).

58. GOERING, "When and Where »:42-43. Grosseteste a accumulé des bénéfices en Angleterre pendant cette décennie, mais ce pourrait être pour soutenir sa carrière académique, sans devoir nécessairement résider de manière continue en Angleterre. Alexandre de Halès, théologien parisien, percevait également des bénéfices ecclésiastiques en Angleterre.

59. GOERING, « When and Where » : 44-46.

60. Toutefois, D. A. CALlus, «Philip the Chancellor and the De Anima ascribed to Robert Grosseteste ", Mediaeval and Renaissance Studies, 1, 1941/ 1943, p. 105-127, a rejeté l'attribution à Grosseteste précisément à cause des liens avec Philippe le Chancelier (qui impliquent que Grosseteste aurait compilé ce traité vers 1235, à une époque où il était en pleine maturité intellectuelle).

61. C. M. cullen, "Alexander of Hales ", in LAGERLund H. (éd.), Encyclopedia of Medieval Philosophy: Philosophy between 500 and 1500, Dordrecht, 2011 : 62-64. Dans ses prolégomènes à l'édition de la Summa theologica, V. Doucet a essayé de déceler la contribution des différents franciscains à sa rédaction (ALEXANDRI DE HALES, Summa Theologica seu 'Summa fratris Alexandri' lib. III, Quaracchi, 1948).

62. Chartularium universitatis Parisiensis, éd. DENIFLE, CHATELAIN E. 1889, t. I, $\mathrm{n}^{\circ} 286: 328$.

63. R. J. LONG, T. B. NOONE, "Fishacre and Rufus on the Metaphysics of Light: two Unedited Texts ", in HAMESSE J. (éd.), Roma, magistra mundi. Itineraria culturae medievalis. Mélanges offerts au Père L. E. Boyle à l'occasion de son $75^{e}$ anniversaire, Louvain-la-Neuve, 1998, p. 517-48: 519, n. 16 et 531, n. 8. Le texte de la questio est réédité en tant qu'Appendix B dans RICHARD FISHACRE, In secundum librum Sententiarum. Part 2 : dist. 21-44. Appendices, éd. LONG 2011 : 333-37.

64. Il s'agit de London, British Library, Royal 10.B.VII, qui contient la questio à la fin du commentaire, et Paris, B.n.F. lat. 15754, dans lequel la questio est placée dans le livre II, dist. 13.

65. Sur les deux versions principales du Speculum maius, voir M. PAULMIER-FOUCART, « Le plan et l'évolution du Speculum maius de Vincent de Beauvais : de la version bifaria à la version trifaria", in MEIER C. E. (éd.), éd., Die Enzyklopädie im Wandel vom Hochmittelalter bis zur frühen Neuzeit, München, 2002 (Münstersche Mittelatler-Schriften, 78), p. 245-68.

66. E. Frunzeanu étudie la question du Memoriale rerum difficilium et des sources qui le composent, pour proposer une édition de ce texte d'après les manuscrits du Speculum naturale de Vincent de Beauvais. 
67. Sur cette influence, voir entre autres DALES, «The Influence of Grosseteste's Hexaemeron on the Sentences Commentaries of Richard Fishacre OP and Richard Rufus of Cornwall OFM », Viator, 2, 1971, p. 271-300.

68. Le texte de Richard Rufus qui résume l'argumentaire de Fishacre est édité par Long et Noone, «Fishacre and Rufus on the Metaphysics of Light »: 537-548, à la suite de la questio de luce de Fishacre.

69. Sur ce genre de manuscrits, voir entre autres: F.-M. HENQUINET, « Un recueil de questions annoté par S. Bonaventure », Archivum Franciscanum historicum, 25, 1932, p. 553-55 ; F. PELSTER, "Quästionen des Franziskaners Richardus Rufus de Cornubia (um 1250) in Cod. VII C. 19 der Nationalbibliothek Neapel und Cod. 138 der Stadtbibliothek Assisi », Scholastik, 14, 1939, p. 215-33 et P. RAEDTS, Richard Rufus of Cornwall and the Tradition of Oxford Theology, Oxford, 1987, p. 64-114. R. J. Long a discuté à nouveau de ces recueils de questions dans sa communication au XII ${ }^{\text {th }}$ International Congress of Medieval Philosophy. Universality of Reason - Plurality of Philosophies in the Middle Ages, Palermo, 2007 : « The Anonymous De anima of Assisi, Biblioteca Comunale ms. $138 »$.

70. Le manuscrit est numérisé sur le site de la Società Internazionale di Studi Francescani d'Assise : http://www.sisf-assisi.it/digitalizzazione.htm.

71. S. DONATI, «Un nuovo testimone dello Scriptum super Metaphysicam di Riccardo Rufo di Cornwall (Salamanca, Bibl. Univ., Ms. 2322) », Bulletin de philosophie médiévale, 45, 2003, p. 31-60.

72. P. SAENGER, "The British Isles and the Origin of the Modern Mode of Biblical Citation", Syntagma. Revista del Instituto de Historia del Libro y de la Lectura, 1, 2005, p. 77-115 : 82.

73. Sur les particularités de chacune de ces concordances et sur l'identité de leurs supposés auteurs (Hugues de St-Cher, Richard de Stavensby et Conrad d'Halberstadt), voir R. H. ROUSE - M. A. ROUSE, «The Verbal Concordance to the Scriptures ", Archivum fratrum praedicatorum, 44, 1974, p. 5-30.

74. De carduo - IV Regum XIVb (DPR XVII.36:69, 1. 18) ; de palma - Canticorum Va (DPR XVII.116 : $173,1.98)$; de sepe - Ecclesiastici XXXVIg (DPR XVII.152 : 215, 1.6); de thimiamate - Exodi XXXg ( DPR XVII.172, p.232, 1.4). Nous avons pu constater que ce même système est utilisé par Barthélemy pour l'Anticlaudianus d'Alain de Lille également (de ysopo - Alanus libro IVf, DPR XVII.85 : 125, 1. 26-27).

75. Dans le cas du Tractatus d'Etienne de Bourbon, qui est tenu pour avoir employé assez précocément le système des concordances, les copistes n'ont pas ensuite rendu correctement ces références (SAENGER, « The British Isles » : 91-92).

76. DPR VIII.2, 1.7-18: Celum autem visibile est multiplex, sicut dicit Glossa super Deuteronomii $X f$. ibi 'En celum domini Dei tui est et celum celi'. Septem sunt celi, scilicet aereum, ethereum, olimpium, igneum, firmamentum, aqueum, empyreum celum, celum scilicet angelorum. Celum itaque aereum vocatur medium aeris interstitium quod a grossis vaporibus aque et terre non per omnia est infectum. Unde illa pars aeris purior et ab inferioribus qualitatibus impermixtior propter sui puritatem et dyaphanitatem sive naturam transparentem celi nomine nuncupatur, sicut dicitur super Matthei XIII 'Volucres celi comederunt illud et cetera'. Dans tous les manuscrits des concordances d'Hugues et de Richard que nous avons consultés (pour Hugues: München, Bayer. Staatsbibl., Clm 14060; Praha, Narodni Knihovna V.C.15; Troyes, Médiathèque patrimoniale 938 et 1603 ; pour Richard : Assisi, Bibl. comunale 42), le verset du Deutéronome est placé sous la lettre $e$ (et non pas $f$ ) du chapitre X. La troisième concordance, entreprise probablement elle aussi à Paris, avant 1286, n'indexe pas ce verset.

77. La teneur du texte biblique (volucres celi comederunt illud) indiquerait une confusion entre le texte de Matthieu (13.4: et venerunt volucres et comederunt ea) et celui de Luc ( 8.5 : et volucres celi comederunt illud). Pourtant, plusieurs manuscrits de l'évangile de Matthieu ont le texte volucres celi comederunt ea ou illa, mais il nous a été impossible de repérer le manuscrit qui comporte le texte cité par Barthélemy et la postille d'Hugues sur le Deutéronome. La postille sur Matthieu du 
manuscrit Orléans, Bibl. municipale 28 (25), daté de 1239, a le texte : et venerunt volucres celi et comederunt ea (p.120b). Ce n'est que chez Thomas d'Aquin que nous avons repéré le verset de Matthieu dans les mêmes termes que sous la plume de Barthélemy (Super evangelium Iohannis reportatio, c. 6, lectio 4, n. ${ }^{\circ} 909$ et Super evangelium Matthei reportatio, c. 13, lectio 1, n. ${ }^{\circ} 1088$ ).

78. Glossa ordinaria Deut. X (ms. Orléans, Bibl. municipale 32 (29), vers 1235-1248, p. 417 ; PL 113, c. 462b) : «Celum celi : Quidam tres dicunt esse celos : aereum, ethereum et sydereum. Nonnulli septem : primum aereum, secundum ethereum, tertium olimpum, quartum igneum, quintum firmamentum, sextum aquarum, septimum angelorum"; HUGO DE SANCTO-CARO, Postilla super totam Bibliam, Deut. X (ms. Reims, Bibl. municipale 164, f. 132ra) : «celi celi: quidam dicunt tres celos esse: aereum, ethereum, sydereum. Nonnulli VII : primum aereum, scilicet sub Luna, ubi habitant aves et etiam demones, unde Matth. XIII 'volucres celi comederunt illud'; secundum ethereum, scilicet in lunaris globi altitudine, tertium olimpum sub Sole, quartum igneum supra Solem, quintum firmamentum quod idem est quod sidereum, sextum aqueum quod est cristallinum, septimum angelorum sive empireum ».

79. La postille d'Hugues de St-Cher était probablement entièrement disponible avant 1239, date à laquelle Jean, abbé de St-Benoît-sur-Loire, en fait faire une copie (ROUSE, Rouse, "The Verbal Concordance $»: 8$ n. 11). Pour la description de ces manuscrits, voir E. PELLEGRIN, J.-P. BOUHOT, Catalogue des manuscrits médiévaux de la Bibliothèque municipale d'Orléans, Paris, 2010, p. 26-31.

80. SAENGER, «The British Isles »: 87-91, qui ne discute pourtant pas les réserves exprimées par les époux Rouse au sujet de la présence de ce système chez Thomas Gallus.

81. Thomae Galli Explanatio in libros Dionysii, éd. LAWELL 2011 ; D.A. LAWELL, "Thomas Gallus's Method as Dionysian Commentator: A Study of the Glose super Angelica Ierarchia (1224), with considerations on the Expositio librorum beati Dionysii ", Archives d'Histoire doctrinale et littéraire du Moyen Âge, 76, 2009, p. 89-117.

82. Voir par exemple la table des occurrences de plusieurs notions, que Thomas Gallus a insérée dans le commentaire du De divinis nominibus (Thomae Galli Explanatio in libros Dionysii : 95-98).

83. éd. CORTESI 1962.

84. L. THORNDIKE, A History of Magic and Experimental Science, New York, 1923, II, p. 401-435 : 418. L'identification de Misael avec Messahalla est réaffirmée dans la brève présentation du manuscrit Venezia, Bibl. Marciana VIII.69, qui contient entre autres des extraits de Barthélemy l'Anglais ( THORNDIKE, «Notes upon some Medieval Astronomical, Astrological and Mathematical Manuscripts at Florence, Milan, Bologna and Venice ", Isis, 50, 1959, p. 33-50 : 46). Nous revenons plus loin sur les extraits contenus dans ce manuscrit. Voir aussi, ci-dessous, l'analyse des extraits empruntés à Zael via Raymond de Marseille.

85. G. M. EDWARDS, The Liber Introductorius of Michael Scot, Ph.D. Diss., Univ. of Southern California, 1978. [édition du Prohemium]. Le renvoi est fait aux pages et aux lignes des éditions respectives.

86. DPR I, 1-2; Michel Scot, Liber introductorius, 1. 8, éd. Edwards 1978: 74, 1. $8:$ ut probatur in principio decretalium; éd. Edwards : 105, 1. 5-6 : in lege canonica decretalis, citation d'Innocent III, la même que celle que reprend Barthélemy, qui continue jusqu'à la p. 108.

87. Les registres de Grégoire IX, éd. AUVRAY 1896, t. I, nº 2083 : 1125-26.

88. Dans les registres de Grégoire IX, l'éditeur résume le texte de la lettre 2084: in eundem modum scriptum est pluribus aliis. Dans l'introduction à son édition des Décrétales, E. Friedberg précise, mais sans donner des détails, que la lettre du pape Grégoire IX fut adressée le 5 septembre 1234 aux universités de Bologne et de Paris (Corpus iuris canonici, II, Leipzig, 1879, réimp. Graz, 1959 : IX).

89. Voir la recension par FRUNZEANU de: «Barthélemy l'Anglais, De proprietatibus rerum, t.1, introd. B. Van den Abeele, Prohemium, éd. H. Meyer, Liv. I, éd. M. W. Twomey, Liv. II, éd. B. Roling, Liv. III et IV, éd. R. J. Long ; t. 6, Liv. XVII, éd. I. Ventura, Turnhout, Brepols, 2007 », Le Moyen Âge, 3-4, 2009, p. 617-21. 
90. I. Draelants remercie Charles Burnett d'avoir attiré son attention sur cette équivalence linguistique.

91. F. SEZGIN, Geschichte des arabischen Schrifttums, VII. Astrologie - Meteorologie und Verwandtes bis ca. $430 \mathrm{H}$, Leiden, 1979:125-128. Cet auteur était bien connu de Michel Scot qui le mentionne comme astrologue dans le Liber introductorius. Les œuvres astrologiques de Zael sont aussi répertoriées dans le chapitre 6 du Speculum astronomiae attribué à Albert le Grand.

92. La forme « Thetel » est associée à un livre des sceaux lapidaires qui a été exploité par tous les compilateurs naturalistes du XIII ${ }^{\mathrm{e}}$ siècle (Arnold de Saxe, Barthélemy l'Anglais, Thomas de Cantimpré, Vincent de Beauvais) et, par leur biais, par Albert le Grand également dans le De mineralibus. Toutefois, on ne conserve plus la version arabe du texte (SEZGIN, Geschichte des arabischen Schriftums : 128).

93. L'enquête pourrait être poussée également du côté des astrologues anglais, comme Roger de Hereford, auteur de plusieurs œuvres sur les jugements astrologiques dont les dépendances n'ont pas encore été élucidées : un Liber de quatuor partibus iudiciorum astronomie et un Liber de tribus generalibus iudiciis astronomie, tous deux inédits, qui pourraient être d'après R. French deux parties d'une même œuvre principale (R. FRENCH, «Foretelling the Future: Arabic Astrology and English Medicine in the Late Twelfth Century ", Isis, 87/3, 1996, p. 453-480: 466, n. 42). La source principale de Roger de Hereford est Abu Ma'shar, qu'il a connu dans la traduction donnée par Hermann de Carinthie, même si Roger ne le cite jamais explicitement. Voir aussi la thèse discutable de N. WHYTE, Roger of Hereford's Liber de astronomice (sic) iudicandi: A Twelfth-Century Astrologer's Manual, M. Phil. Diss., Clare College, 1991. Le sondage que nous avons réalisé dans les traductions des Tables d'al-Khwārizmi par Adélard de Bath, revues par Robert de Chester (qui a aussi traduit les Iudicia d'al-Kindī), montre qu'il y a des points communs entre le Liber de astronomia iudicandi de Roger de Hereford, les Tables d'al-Khwārizmi, les traductions de 'Jafar Indus' par Hugues de Santalla et l'Introductorium de Zael. Ces rapprochements s'expliquent par les échanges qui auraient pu avoir lieu au XII siècle: Hugues de Santalla aurait été l'associé d'Hermann de Carinthie, qui a étudié l'astronomie avec Robert de Ketton au nord de l'Espagne ( BURnetT C., PIngree D. (éd.), The Liber Aristotilis of Hugo de Santalla, London, 1997, (Warburg Institute Surveys and Texts, 26) : 1-2). Par ailleurs, Petrus Alfonsi a, comme Adélard de Bath, établi une version des Tables d'al-Khwārizmi (les chapitres de P. Alfonsi portent la date de 1116, ceux d'Adélard celle de 1126, comme nous l'a fait remarquer obligeamment Ch. Burnett) et a enseigné l'astronomie en Angleterre et en France.

94. Il est probable que cette traduction ait été menée par Iohannes Hispalensis et Limiensis. Sur cette oeuvre, voir JUSTE, CCAL, I, Les manuscrits astrologiques latins conservés à la Bayerische Staatsbibliothek de Munich, Paris, $2011: 31$ et n. 20 ; sur le manuscrit Paris, B.n.F. lat. 16204, voir PINGREE, "The Diffusion of Arabic Magical Texts in Western Europe ", in La diffusione delle scienze islamiche nel Medio Evo Europeo, SCARCIA AMORETTI Biancamaria (éd.), Rome, 1987, p. 57-102, notamment 80-88 et 100-102. Ce manuscrit fut copié par un des scribes de Richard de Fournival. Les 27 traités qu'il contient sont mentionnés dans le Speculum astronomie, à l'exception des De revolutionibus annorum nativitatum d'Albumasar (353b-369b), Liber sigillorum de Thetel (500a-505a) et De lapidibus d'Azareus (505a-507a).

95. Le début de l'Introductio au Liber iudiciorum (Iudicia) de Zael est édité par BURNETT, «A Group of Arabic-Latin Translators Working in Northern Spain in the Mid-12th Century ", Journal of the Royal Asiatic Society of Great Britain and Ireland, 1977, p. 62-108:103-104, avec en regard la traduction d'Hermann de Carinthie et la version du Liber novem iudicum.

96. L'édition de Venise que nous avons utilisée (1493; GW M36394) contient les sections suivantes : Liber quadripartiti Ptholemei, f. 2r-106v; Centiloquium eiusdem; f. 107r-116v; Centiloquium Hermetis, f. 117r-118r ; Liber Hermetis de stellis beibeniis, f. 118r-119r ; Centiloquium Bethem, de horis planetarum et de significatione triplicitatum ortus, f.119r-120r; Almansoris iudicia seu propositiones, 
f. 120v-122r ; Zahel Introductorium de principiis iudiciorum: de interrogationibus, f. 122v-138r ; de electionibus, f. $138 \mathrm{v}-141 \mathrm{v}$; de significatione temporis ad iudicia, f. $141 \mathrm{v}-142 \mathrm{v}$; Messahallach de receptione planetarum (de interrogationibus), f.143r-148r); Epistola eiusdem de coniunctionibus planetarum, f. 148r-149r ; Eiusdem de revolutionibus annorum mundi, f. 149r-152r.

97. Inc. Signorum alia sunt masculina alia feminina. Manuscrit le plus ancien : London, British Libr., Harley 5402, XII ${ }^{\text {e }}$ s., f. 1r-14v (manuscrit numérisé et accessible sur le site de la British Library).

98. JUSTE, Les manuscrits astrologiques, $2011: 51-52$.

99. Le manuscrit Paris, B.n.F., lat. 16208, f. 13ra-26rb contient ce texte, à savoir la longue introduction et les jugements astrologiques, dans une copie du XII ${ }^{\mathrm{e}}$ siècle. Toutefois, le texte du manuscrit de Munich, Bayer. Staatsbibl., Clm 51 contient trois parties (II : f. 63va ; III : f. 65rb, de exposicione et fortitudinis).

100. Decem continens tractatus astronomie sive Liber astronomicus, Augsburg, E. Ratdolt, 1491 (GW n ${ }^{\circ}$ 4643). Bonatti cite souvent l'autorité d'Adila (ou sacerdos Adila) lorsqu'il présente les propriétés des planètes et des signes. Les termes de ces descriptions se rapprochent fortement de la famille des Iudicia. Adila apparaît aussi dans le Liber iudiciorum de Raymond de Marseille : Sed Albumasar atque Adila ceterique quorum auctoritas potior habetur (Paris, B.n.F. lat. 16208, f. 24ra). Selon Ch. Burnett, Adila est une forme abrégée d'Alcabitius («Ten or Forty? A Confusing Numerical Symbol in the Middle Ages» in Mathematics Celestial and Terrestrial. Festschrift für Menso Folkerts zum 65. Geburtstag, p. 81-90). Pourtant, chez Bonatti, les citations associées au nom d'Adila suivent parfois directement des citations mises sous l'autorité d'Alcabitius, ce qui laisse entendre que Bonatti n'identifiait pas l'un avec l'autre. De plus, les passages associés par Bonatti au nom Adila correspondent parfois à la lettre au texte de Raymond de Marseille (p. ex. la description de Saturne, Decem continens tractatus astronomie, tract. III, pars I, c. I, f. $45 \mathrm{v}$ et Liber iudiciorum, Paris, B.n.F., lat. 16208, f. 13rb). Ailleurs, au sujet des propriétés de la cinquième maison, Bonatti conjoint Adila à l'autorité de "Vuellius» (déformation probable de Vettius Valens) et "Alchaiat»: Dixit Adilla quod significat gaudium et vestimenta. Et dixit Vuellius quod significat cartas, libros, nuncios atque novellas. Et dixit Alchaiat quod significat omne in quo habetur fiducia, honorem, petitionem, mulierem, amiciciam et esse civium et fructus hereditatis (Decem continens tractatus astronomie, tract. II, pars III, c. 5, f. 31v). Ce passage apparaît à la lettre chez Raymond de Marseille, les autorités insérées par Bonatti n'y figurant pourtant nulle part (Paris, B.n.F., lat. 16208 , f. 15vb). Bonatti a-t-il eu accès à un manuscrit de Raymond doté de marqueurs dans les marges?

101. DRAELANTS, FRUNZEANU, «Sur les traces du De motibus ».

102. Sur ces deux textes et leurs copies manuscrites, voir JUSTE, CCAL I. Les manuscrits astrologiques latins, et BURNETT, « Aristotle as an Authority on Judicial Astrology », in MEIRINHOS J. F., WEIJERS O. (éd.), Florilegium mediaevale. Etudes offertes à Jacqueline Hamesse à l'occasion de son éméritat, Louvainla-Neuve, 2009, p. 39-62.

103. Un vif merci à David Juste de nous avoir transmis la transcription du texte des Iudicia Aristotelis.

104. Chez Barthélemy suivent les extraits de l'autre groupe de Iudicia Ptolomei, empruntés à Arnold de Saxe: De hoc Saturno dicit Ptolomeus: Sub Saturno sunt Capricornus et Aquarius, eius dominium est in Libra, sed in Ariete regnum abstrahit, etc.

105. Al-Qabisīi (Alcabitius), The Introduction to Astrology, éd. BURNETT, YAMAMOTO, YANo 2004. Cf. F. CARMODY, Arabic Astronomical and Astrological Sciences in Latin Translation. A Critical Bibliography, Berkeley-Los Angeles, 1956 : 144-149 (Carmody répertorie 106 manuscrits, Burnett 200). Résumé du contenu de l'Introductorius dans J. D. NORTH, Chaucer's Universe, Oxford, 1988, p. 192-254 (règles d'astrologie arabo-latine). Il est aussi une source importante de l'astrologie de Michel Scot dans son Liber introductorius et est utilisé par Vincent de Beauvais dans le Speculum naturale, XV.39-42. Le texte suivi par Vincent de Beauvais s'apparente à la famille «Beta » de l'Introductorius, famille 
qui se rapproche de la version arabe du texte. Voir aussi BURNETT, «Vincent of Beauvais, Michael Scot and the 'New Aristotle' ", in PAULMIER-FOUCART M., LUSIGNAN S. (éd.), Vincent de Beauvais. Lector et compilator, Grâne, 1997, p. 189-213 : 209, n. 23.

106. La citation mise sous le marqueur De motibus planetarum pourrait être rapprochée de l' Epistola de rebus eclipsium et coniunctionibus planetarum de Messahalla (voir DRAELANTS, Arnold de Saxe : 214).

107. P. KUNITZSCH, «Zur Tradition der 'Unwettersterne'», Zeitschrift der Deutschen Morgenländischen Gesellschaft, 122, 1972, p. 108-117 ; BURNETT, «An Unknown Latin Version of an Ancient Parapegma: The Weather-Forecasting Stars in the Iudicia of Pseudo-Ptolemy ", in Making Instruments Count: Essays on Historical Scientific Instruments presented to Gerard L'Estrange Turner, R.G.W. ANDERSON et al. (éd.), Aldershot, 1993, p. 27-41; G. BOS, C. BURNETT, Scientific Weather Forecasting in the Middle Ages: The Writings of Al-Kindī, London-New York, 2000 ; BURNETT, «Late Antique and Medieval Latin Translations of Greek Texts on Astrology and Magic ", in The Occult Sciences in Byzantium, MAGdalino P., MAVRoudi M. (éd.), Genève, 2006, p. 325-359 ; D. LeHouX, Astronomy, Weather, and Calendars in the Ancient World, Cambridge-New York, 2007.

108. A. BORST, Astrolab und Klosterreform an der Jahrtausendwende (Sitzungberichte der Heidelberger Akademie der Wissenschaften, Phil.-hist. Klasse, 1/1989), Heidelberg, 1989 ; P. KUNITZSCH, E. DEKKER , "The Stars on the Rete of the so-called 'Carolingian Astrolabe' », in Casulleras J., Samsó J. (ed.), From Baghdad to Barcelona. Studies in the Islamic Exact Sciences in Honour of Prof. Juan Vernet, Barcelona, 1996, p. 655-672; BURNETT, «King Ptolemy and Alchandreus the Philosopher: The Earliest Texts on the Astrolabe and Arabic Astrology at Fleury, Micy and Chartres ", Annals of Science, 55, 1998, p. 329-368; KUNITZsCH, «Zur Problematik der Astrolabsterne: eine weitere unbrauchbare Sterntafel », Archives internationales d'histoire des sciences, 43, 1993, p. 197-208; A. BORRELLI, Aspects of the Astrolabe: 'architectonica ratio' in tenth- and eleventh-century Europe, Stuttgart, 2008 ; RAYMOND DE MARSEILLE, Liber cursuum planetarum, éd. D'ALVERNY, BURNETT, POULLE 2009 (avec compléments I. CAIAzzo, Ch. BURNETT, "New Manuscripts of On the Astrolabe by Raymond of Marseille», Scriptorium, 65, 2011, p. 338-349) ; D. A. KING, Astrolabes from Medieval Europe, Farnham, 2011.

109. KUNITZSCH, Typen von Sternverzeichnissen in astronomischen Handschriften des zehnten bis vierzehnten Jahrhunderts, Wiesbaden, 1966 ; KUNITZSCH, The Arabs and the Stars. Texts and Traditions on the Fixed Stars, and their Influence in Medieval Europe, Northampton (Variorum Reprints), 1989. Les listes d'étoiles que P. Kunitzsch a mises en rapport avec Albumasar ou avec l'Epitome totius astrologiae écrit en 1142 et attribué à Jean de Séville (Iohannes Hispalensis) font usage d'une terminologie latine pour désigner les constellations ( $« \mathrm{Abu}$ Ma`šar, Johannes Hispalensis und Alkameluz », Zeitschrift der Deutschen Morgenländischen Gesellschaft, 120, 1970, p. 103-125. Pour le rapport avec les écrits d'Albumasar, voir aussi DRAELANTS, Arnold de Saxe, 2000 : 212-214.

110. Merci à Arnaud Zucker pour les remarques et les compléments d'information qu'il nous a fournis au sujet de l'astronomie antique.

111. Étymologies, III.26.2, éd. GASPAROTTO 2009 : 88.

112. DPR VIII.1, 1.16: Secundo modo dicitur mundus universitas eorum que celi ambitu continentur [...] de quo dicitur 'Mundus per ipsum factus est'; VIII.1, 1.117-118: [...] dicit Glossa ibi 'Celum autem et terra transient et cetera' (Mattheus); VIII.2, 1.8-9 : Celum autem visibile est multiplex, sicut dicit Glossa super Deuteronomii Xf. ibi 'En celum domini Dei tui est et celum celi'; VIII.2, 1.14-18 : Unde illa pars aeris purior et ab inferioribus qualitatibus impermixtior [...] celi nomine nuncupatur, sicut dicitur super Matthei XIII 'Volucres celi comederunt illud et cetera'; VIII.2, 1. 31-32: Secundum Gregorium autem super locum illum Iob 'Intuere celum et contemplare ethera'; VIII.3, 1.4-5: Aquas enim esse super celos collocatas Scripture divine auctoritas nobis tradit ; VIII.3, 1.34-35 : Et tamen scriptum est Iob XXXVIII: 'Qui facit concordiam in sublimibus suis'; VIII.10, 1.253-258: Has itaque XII signorum celi proprietates et occultas virtutes secundum astrologorum iudicium huic opusculo interserui, estimans utile esse legentibus eorum nomina in 
Scriptura breviter et pariter invenire quid de eis senserint antiqui et quare eis talia nomina imposuerunt astrologi siderum inspectores.

113. Au début du c. 49 , intitulé "Quod ex significationibus stellarum in conceptionibus destituitur omnis astrologica scientia»: Zehel Embenidis Iudeus genere Arabs natione, optimus astrologus, in commento suo De astrologia (M. H. MALEWICZ, «Le Libellus de efficatia artis astrologice: Traité astrologique d'Eudes de Champagne, XII ${ }^{\mathrm{e}}$ siècle », Mediaevalia Philosophica Polonorum, 20, 1974, p. 3-95).

114. DPR, $1601: 469$ : Has et multas alias proprietates habet materia, quas superfluum esset hic recitare. Quere tamen supra libro octavo de bonitate mundi.

115. Monde - VIII.1, 1. 115 ; firmament - VIII.2, 1. 77 et 1. 237, VIII.22, 1. 34, VIII.23, 1. 19 ; lumière VIII.5, 1. 4, VIII.17, 1. 72, VIII.29, 1.126. Il convient de signaler la seule occurrence de l'adjectif eternus, dans une citation tirée de Martianus Capella (eternis celi raptibus, DPR VIII.1, 1. 44), où eternus doit être compris au sens de continuel davantage que d'éternel. D'ailleurs, lorsqu'il est question du mouvement du firmament, Barthélemy reprend la même image et utilise l'expression sempiternis raptibus (DPR VIII.23, 1. 19).

116. DPR VIII.1, 1.27-34: Mundus igitur, ut dicit Martianus, est universitas creatorum conglobata in spere modum. Nam mundana superficies spericam et circularem habet speciem et figuram. Nec fuit, ut dicit Martianus, alia figura mundo ita congrua sicut orbicularis et rotunda, et hoc propter universitatis perfectionem et propter illam quam habet in esse perpetuo cum suo opifice, qui fine caret et principio, configurationem ; DPR VIII.6, 1. 2-4: Spera celi, ut dicit Isidorus, est species quedam in rotundum formata ab eodem in idem circa suum centrum equis spatiis circulariter revoluta.

117. Le terme circulus est utilisé pour désigner autant les cercles célestes que ceux des planètes, alors qu'orbis est plutôt réservé aux cercles des planètes. Il existe des endroits où les deux termes sont rapportés aux planètes : DPR VIII.2, 1.34 et 189 : orbis et circulus planetarum.

118. La source que Barthélemy reprend pour décrire la Voie Lactée reste fidèle à Macrobe pour dire qu'elle croise le cercle du zodiaque dans le signe du Cancer et du Capricorne : DPR VIII.8, 1. 2-6: Galaxias est circulus celestis ceteris circulis celi pulcrior et candidior, incedens per medium celi, incipiens ab oriente usque ad septentrionem, per Cancrum et Capricornum procedens iterum ad punctum suum. Or, dès le IX $\mathrm{I}^{\mathrm{e}}$ siècle, on avait corrigé cette erreur pour y substituer les Gémeaux et le Sagittaire (M. ARMISEN-MARCHETTI (éd.), Macrobe. Commentaire au songe de Scipion, Paris, 2001, lib. I : 168, n. 261 ; B. S. EASTWOoD, Ordering the Heavens. Roman Astronomy and Cosmology in the Carolingian Renaissance, Leiden/ Boston, 2007 : 69-70).

119. Le chapitre consacré aux cercles célestes est mis sous l'égide d'Isidore (DPR VIII.7, 1. 37). Or, comme nous l'avons déjà mentionné, Isidore reprend in extenso dans ses Étymologies les définitions données par Hygin dans son De astronomia (Etym., III.44 et XIII.6), alors que le texte compilé par Barthélemy est fondé sur Macrobe et Martianus Capella. Guillaume de Conches avait déjà combiné ces deux dernières sources dans la Philosophia et son exposé a été plus tard complété par l'Experimentator par des termes définissant les parallèles. C'est la version de l' Experimentator que Barthélemy reprend à cet endroit.

120. Cependant, dans la description de la sphère armillaire que Ptolémée fait dans l'Almageste (5.1), l'armille mobile de réglage du méridien figure parmi les 7 armilles.

121. DPR VIII.2, 1. 125-142: Omne autem quod sub ipso est, ut dicit Rabanus, virtuti eius motive obedit ordine uniformi et imperturbato. Virtus quoque eius motiva, sicut ipsemet dicit super expositione Geneseos, extendit se usque ad hec inferiora elementa. Unde et ignem superiorem creditur secum circumrotare. Pervenit quoque huius motive virtutis actio usque ad aerem et etiam usque ad aquas, in quibus quo ad fluxum et refluxum in parte maxima facit exuberationem. Hec autem tria elementa, scilicet aer, ignis et aqua, obediunt cuidam motui celi, sed non uno modo neque uno ordine ipsum imitantur, sed secundum maius et minus, quia quod superius est et levius et purius obedit magis, et quod minus est, tale sequitur tardius et obedit minus. Terra huic celesti virtuti et motui non obedit, ut ab eo suscipiat localem motionem, quamvis ad producendas ex se species diversas suscipiat impressiones. 
122. DPR VIII.2, 1.219-235: [...] dicitur in libro De celo et mundo in fine quod celum motus sui continuitate inflammat illud super quod movetur, unde et ether ex motu celi ignitur. Motus enim causat calorem. Unde ibidem dicitur per se et sensibiliter invenitur quod ex motu fit calor et ex quiete frigiditas. Et ideo calor materias emollit et dissolvit, frigiditas vero aggregat et constringit. Unde partes elementares motui celi vicinius existentes ceteris partibus mundi inferioris sunt calidiores, ut patet in igne et in aere. In tertio autem elemento, scilicet in aqua, est motus debilitatus, et ideo in ea est frigiditas, sed non in ultimo. In quarto autem, scilicet in terra, propter nimiam elongationem a motu celi fit constrictio in ultimo propter caloris absentiam et dominium frigiditatis. Et ideo terra permanet quiescens et perennis, ut non moveatur, sicut dicit Aristoteles ibidem.

123. L'immobilité de la Terre au centre de l'univers est une thèse largement acceptée depuis l'Antiquité (BAKHOUCHE, Les textes latins d'astronomie : 109-111).

124. DPR VIII.2, 1.55-57 : Immo, ut dicit Basilius in Hexameron, primo philosophi linguas suas corroderent, quam plures celos esse consentirent.

125. Sur les partisans d'un cosmos à ciel unique, voir E. GRANT, Planets, Stars, and Orbs. The Medieval Cosmos, 1200-1687, Cambridge, 1996 : 97-100.

126. DPR VIII.2, 1.31-35: Secundum Gregorium autem super locum illum Iob 'Intuere celum et contemplare ethera' et cetera, nomine etheris intelligitur tota regio a Luna usque ad stellas fixas, in qua sunt orbes et circuli septem planetarum.

127. DPR VIII.2, 1. 35-36: Quere infra proximo de ethere quid super hoc sentiat Martianus.

128. DPR VIII.2, 1.37-51: Alexander enim sic ordinat septem celos, dicens primum celum est luminis dativum, uniforme et non motum, scilicet celum empyreum. Secundum celum esse dicit dativum luminis, uniforme et motum, scilicet aqueum sive cristallinum. Tertium dativum luminis non secundum omnem partem luminositatis uniforme et motum, ut celum stellatum. Quartum est receptivum luminis sine calore, scilicet olimpium. Quintum est receptivum luminis cum calore, scilicet celum igneum. Sextum est receptivum luminis coniunctum cum parte superiori, et dicitur celum ethereum. Septimum est receptivum luminositatis coniunctum cum parte inferiori, scilicet aereum. Et sic videtur vocare Olimpum regionem orbium planetarum, quia illud spatium est continue luminosum.

129. Bonaventure, In II Sent., II, dist. 2, pars 2, dub. 2, éd. Quaracchi 1885 : 85b-86b : Nota tamen, quod in dispositione caelorum sive numero videntur contradicere doctores sacrae Scripturae philosophis. Nam philosophi sphaeras octo esse dicunt, doctores aliqui novem ponunt, aliqui octo, aliqui septem caelos; et ideo sibi ipsis videntur contrariari. Ratio autem huius diversitatis est, quia philosophi nihil de empyreo sunt locuti, nihil etiam de igne et aëre, quando loquuntur de caelis. Scriptura sacra etiam aërem caelum appellat, et aquas supra caelum et ipsum empyreum et ipsum Deum. Ratio autem diversitatis inter doctores ex hoc venit, quod quidam numerant caelum spirituale cum corporalibus, ut Beda; quidam vero omittunt ulterius; quidam dividunt firmamentum in duo, scilicet in caelum sidereum et planetarum; quidam coniungunt. Similiter quidam dividunt aëreum in duos, quidam coniungunt; similiter de igneo. Tamen secundum communiorem computationem novem ponuntur caeli, largissime accipiendo, scilicet aëreum, aethereum, igneum, olympicum, caelum planetarum, firmamentum, aqueum, empyreum et caelum Trinitatis.

130. ALBERTUS MAGNUS, Summa de creaturis, pars I, tract. III, quaest. XIV : éd. Borgnet 1895 : 430 : Si autem queritur de ratione numeri celorum secundum Rabanum, dicendum quod natura communis omnibus celis secundum eum est duplex, scilicet natura continendi circulariter et natura perspicui. Unde quot modis contingit multiplicari perspicuum continens circulariter, tot erunt celi. Perspicuum autem tale aut erit receptivum luminis aut dativum. Si dativum aut est dativum uniforme aut non uniforme. Item aut motum aut non motum. Si dativum uniforme non motum, tunc est celum empyreum. Si autem est dativum uniforme motum, tunc est chrystallinum sive aqueum. Si autem dativum non uniforme motum, tunc erit firmamentum. Quarta vero combinatio non potest esse, scilicet non uniforme, non motum, quia supra probatum est, quod omne quod non est uniforme movetur. Et ita in quinta essentia secundum Rabanum non sunt nisi tres celi. Si autem est receptivum luminis aut separabiliter aut inseparabiliter. Si inseparabiliter aut secundum circulum superioris superficiei aut inferioris. Si primum, tunc est igneum, 
quia ignis est elementum receptivum luminis inseparabiliter. Si autem secundum superficiem inferiorem, tunc est olimpium. Si vero est receptivum luminis separabiliter, sicut aer, qui illuminatur ex presentia luminosi et obtenebratur absentia eiusdem, aut secundum superius vel inferius. Si secundum superius, tunc est ethereum, quia Rabanus superiorem regionem aeris vocat etherem. Si autem secundum inferius, tunc est aereum. La différence qui sépare Albert le Grand d'Alexandre de Halès au sujet de l'ordre de l'igné et de l'olympien est discutée par Vincent de Beauvais dans son Speculum naturale (III, 105 , Actor et IV, 8, Actor) où il fait place à l'exposé des deux auteurs.

131. THOMAS DE AQUINo, In II Sent., dist. 14, quaest. 1, art. 4, éd. Opera omnia 1856, t. VI : 508b : Natura enim corporis caelestis est ut separatum sit a corruptione et a contrariis; proprietates autem eius praecipue attenduntur in altitudine situs et claritatis participatione. Si ergo nominemus caelum a natura et proprietate caelesti, sic est triplex caelum; quorum unum est uniforme et immobile, scilicet empyreum; secundum uniforme et mobile, scilicet caelum chrystallinum; tertium difforme in partibus et mobile, scilicet sidereum. Si autem dicatur caelum a proprietatibus caelestibus tantum, sic erit quadruplex caelum. Sunt enim duo elementa, quae simul claritatem lucis participant et altitudinem situs, scilicet aer et ignis, in quorum utroque distinguitur pars superior quantum ad convexum sphaerae ipsius, et pars inferior quantum ad concavum. Pars ergo superior sphaerae ignis dicitur caelum igneum, eo quod ignis ibi purissimus est, propriam naturam servans; pars autem inferior eius caelum olympium dicitur, a quodam monte, qui nominatur Olympus in Macedonia, qui ultra nubes in altum dicitur porrigi; superior vero pars aeris dicitur caelum aethereum, propter inflammationem illius partis ex propinquitate ignis, quia, ut in 1 cael. et mund. dicitur, nomen aetheris designat corpus velocis motus, cito inflammabile vel inflammans; inferior vero pars aeris dicitur caelum aereum, in qua est aer servans proprietatem aeris nobis noti et in usum nostrum venienti.

132. E. Grant rappelle que cet « apparent conflit » a retenu très peu l'attention au Moyen Âge, si ce n'est après la traduction du commentaire de Simplicius sur le De celo (Planets, Stars, and Orbs : 422-428).

133. DPR VIII.2, 1.59-71: Aristoteles in libro De causis elementorum: Celum, inquit, est elementum quintum ab elementis inferioribus discretum naturali proprietate ac distinctum. Neque enim grave est, quia tunc descendere posset, neque leve, quia forsan tunc ascenderet, quia si esset unum ex quatuor vel esset compositum ex quatuor, ingrederetur in ipsum corruptio universaliter vel particulariter. Immo, sicut dicitur ibidem, Creator posuit eum causam et principium generationis et corruptionis. Et ideo necesse fuit ipsum esse ingenerabile et incorruptibile, ne pro querende generationis et corruptionis principio esset abire in infinitum; VIII.22, 1.29-31: Aristoteles autem et alii philosophi, qui ponunt celum esse elementum quintum natura et proprietate ab elementis quatuor discretum.

134. VIII.2, 1.95-112: De istius celi natura dicitur in libro De celo et mundo secundum novam translationem: Celum, inquit, est unum compositum in materia, quoniam continet materiam ex qua et celum nominatur, quod est ultimus incessus totius, scilicet universi. Et sequitur in eodem: Non sunt neque fuerunt neque erunt celi multi. Celum enim est unum perfectum completum, cui non est simile, neque est locus extra celum neque corpus neque vacuitas neque plenitudo neque tempus, quod est numerus motus. Unde illic est vita fixa, scilicet extra ultimum, et est sempiterna que neque deficit neque finitur, et illa est vita vera. Unde idem ibidem: Celum neque fabricatum est neque generatum, sed simplex est, cuius motus est equalis, in quo non est diversitas. Et est motus eius singularis et circularis, cuius motor est spiritus qui movet illud sua voluntate, et continuatur radius celi cum radio ignis, et coniunctus est cum illo ad utilitatem hominum propter permanentiam vite.

135. Nous avons déjà comparé plus haut la teneur de cette phrase chez Barthélemy, Arnold de Saxe et Aristote.

136. DPR VIII.2, 1. 240-241: Nobilitas itaque celi attenditur [...] in situs sublimitate, quia quo ad situm a centro terre maxime distat .

137. DPR VIII.2, 1.59-60 : Aristoteles in libro De causis elementorum: Celum, inquit, est elementum quintum ab elementis inferioribus discretum naturali proprietate ac distinctum. 
138. AUGUSTINUS, Commentaire sur la Genèse au sens littéral, II, 1-5, éd. AGAËSSE, SOLIGNAC 1972 : 147-149, 161 : «Il est une question par contre qui mérite maintenant d'être posée : ce ciel dont il s'agit estil celui qui se trouve au-dessus de toutes les étendues de l'air et même de ces hauteurs où sont placés, le quatrième jour, les corps lumineux et les étoiles? ou bien est-ce l'air lui-même qui est appelé firmament? Beaucoup prétendent en effet que ces eaux ne peuvent, du fait de leur nature, être situées au-dessus du ciel sidéral, attendu que, en vertu de leur propre poids, elles doivent ou s'écouler sur le sol ou rester en suspension dans l'air, à l'état de vapeurs, au voisinage des terres. Il ne convient pas de les réfuter en disant que, eu égard à la toute-puissance de Dieu auquel tout est possible, nous devons croire que ces eaux, fussent-elles aussi lourdes que nous le savons et l'expérimentons, ont été répandues au-dessus de ce ciel matériel qui contient les astres. L'objet actuel de nos recherches en effet est de savoir comment, d'après les Ecritures, Dieu a constitué la nature des choses, non ce qu'il lui plaît de faire en elles ou à partir d'elles pour manifester miraculeusement sa puissance. (...) En tous cas, quelles que soient ces eaux et sous quelque forme qu'elles soient là-haut, ne doutons aucunement qu'elles y soient, car l'autorité de l'Ecriture prévaut contre les ressources de tout esprit humain ».

139. H. R. LEMAY, "Science and Theology at Chartres: The Case of the Supracelestial Waters ", in The British Journal for the History of Science, 10, 1977, p. 226-36.

140. DPR VIII.3, 1. 7-19: Beda tamen dicit quod aque ille celestes non vaporabili tenuitate, sed glaciali soliditate virtute divina super firmamentum sunt suspense, et hoc modo ad impetus firmamenti moderationem vel ad caloris generati ex eius velocissimo motu repressionem. Opinio enim fuit Bede quod celum ignee sit nature, sicut Platonici posuerunt. Unde dicit Beda: Celum est subtilis et ignee nature, rotundum a centro terre equalibus spatiis collocatum. Et ideo forte Bede videbatur quod ideo fuit necesse ibi esse aquas, ut calor ille celestis ad temperantiam duceretur et quod ex celi inflammatione mundus inferior dispendium non pateretur.

141. R. J. Long a synthétisé les différentes réponses apportées par Robert Grosseteste, Richard Fishacre et Richard Rufus: LONG, «The Division of the Waters (Gen 1,6-7): The History of a Conundrum and its Resolution by the Early Oxford Masters ", in EMERY K., FRIEDMAN R. L., SPEER A. (éd.), Philosophy and Theology in the Long Middle Ages. A Tribute to Stephen F. Brown, Leiden, Boston, 2011, p. 41-62 ; voir aussi GRANT, Planets, Stars, and Orbs, 1996 : 332-334.

142. LONG, « The division of the Waters (Gen $1,6-7) »: 62$.

143. Nous reprenons l'édition de ce tableau à F. S. Pedersen dans The Toledan Tables, København, 2002 : 1590-91, tables RA11 et RA11a. A l'exception des exaltations données pour la Tête et la Queue du Dragon, toutes les autres données sont attestées par différents représentants de l'astrologie antique, que ce soit Ptolémée, Pline ou Firmicus Maternus.

144. JUSTE D., Les Alchandreana primitifs : 223.

145. BAKHOUCHE, Les textes latins d'astronomie, $1996: 169-172$.

146. EASTWOOD, Ordering the Heavens, 2007 : 32. La rotondité de la terre est admise et démontrée de longue date à l'époque de Barthélemy. On connaît par exemple les démonstrations qu'en fait Guillaume de Conches, Philosophia (schéma de la terre dans le manuscrit Paris, B.n.F. lat. 11130, f. 47v), en s'appuyant sur Macrobe [cf. B. OBRIST et I. CAIAzzo, éd., Guillaume de Conches. Philosophie et science au XII ${ }^{e}$ siècle, Firenze, 2011, (Micrologus' Library 42), en particulier l'article de P. GAUTIER DALCHÉ sur l'influence de Macrobe pour le problème de la sphère et des antipodes], tandis que Robert Grosseteste, De sphera, cite à ce propos Ptolémée et Thâbit ibn Qurrâ [cf. J. P. cunNInGHAM et M. HOCKNULL, éd., Robert Grosseteste and the Pursuit of Religious and Scientific Learning in the Middle Ages, s. l., 2016, (Studies in the History of Philosophy of Mind 18), p. 12].

147. Sur l'ordre des planètes et leur tempérament, voir W. HÜBNER, «Astrologie et mythologie dans la Tétrabible de Ptolémée d'Alexandrie ", in Sciences exactes et sciences appliquées à Alexandrie (III siècle av. J.-C. - I ${ }^{\text {er }}$ siècle ap. J.-C.), p. 325-45. Voici la nature des planètes (qualités fondamentales) selon Ptolémée : Soleil (chaud-sec), Lune (humide-chaude), Saturne (froid-sec), Jupiter (chaud-humide), Mars (sec-chaud), Vénus (humide-chaude), Mercure (sec-humide) ( 
PTolomaeus, Tetrabiblos, I.4, éd. RoBbins 1940: 35-39). La première qualité domine. Chez Barthélemy, la nature des planètes est un peu différente : la Lune est froide et humide, Mercure est tempéré (DPR VIII.11, 1. 56-68).

148. Cf. Ptolomaeus, Tetr., I.6 : 41. Sur les couleurs des planètes, voir entre autres PLINE, NH, II.79 éd. BEAUJEU 1950 : 34, repris par BEDA, De natura rerum, XV, éd. JONES 1975 : 207 ; RABAN MAUR, De Computo, XLVIII, éd. STEVENS 1979 : 258, et ABBON DE FLEURY, De ratione spere, éd. THOMSON 1985 : 122. 149. Macrobe associe Archimède et Cicéron au modèle chaldéen et Platon au modèle égyptien ( Somn. Scip. I.19. 1-10). Macrobe intervertit en fait les places que Platon, et Chalcidius avec lui, attribuait à Mercure et à Vénus, ce qui plaçait le Soleil entre la Lune et Vénus.

150. L'intérêt pour la question de l'ordre des planètes au Moyen Âge est patent du fait que l'un des compendia astronomiques rédigés à l'époque carolingienne intègre un traité $D e$ ordine planetarum fondé sur Pline, Macrobe et Chalcidius (EASTwOoD, Ordering the Heavens, 2007 : 68-69).

151. B. S. Eastwood, Ordering the Heavens, 2007 : 43-52 étudie la façon dont l'interprétation donnée par Dungal du texte de Macrobe a déterminé la représentation graphique de l'ordre planétaire chaldéen, alors que Macrobe était l'adepte du modèle égyptien.

152. DPR VIII.16, 1. 10-17 : Et dicitur Mercurius quasi medius currens, quia quasi medius est secundum Bedam inter Venerem et Solem. Unde in superiori parte sui circuli coniungitur cum Venere, in inferiori vero convenit cum Sole. Circulus etiam suus in superiori parte sui intrat circulum Veneris, in inferiori autem parte circulum intrat Solis. Quando est in superiori parte sui circuli, melius videtur et minus a Sole obscuratur.

153. DPR VIII.11, 1. 110-114: Nam domus Saturni prima et propria est Capricornus, similiter et Aquarius, domus Iovis Piscis et Sagittarius, domus Martis Aries et Scorpius, domus Veneris Libra et Taurus, domus Mercurii Virgo et Gemini, domus Solis Leo, domus Lune Cancer.

154. Somn. Scip., I, 21.24-27.

155. DPR VIII.11, 1. 81-105: Unde secundum astrologos et etiam secundum Galienum et alios peritos medicos Saturnus materiam conceptam in matrice frigiditate sua et siccitate coagulat et condensat. Iupiter autem secundo mense spiritum et membra tribuit. Tertio mense Mars subtiliat sanguinem et humores condensat et ordinat et componit. Sol in quarto mense vitalem calorem et spiritum epati et cordi tribuit. Venus in quinto mense organa sensibilia et officialia membra, aures scilicet et nasum et huiusmodi, perficit et disponit. Mercurius sexto mense omnia corporis foramina et porosa membra, ut linguam et renes, componit. Luna mense septimo suis limitibus membra dividit et distinguit, et ideo fetus tunc nascitur utilis et salubris. Si vero nasci distulerit usque ad octavum mensem, fetus tunc moritur, et hoc quia virtus Saturni tunc iterum redit, qui utraque qualitate sua, frigida scilicet et sicca, fetum mortificat et constringit. Nono autem mense regnat Iupiter iterum, qui fetus calore suo temperato refovet et custodit, unde partus tunc nascens vitalis erit. Similiter nascens in principio decimi mensis potest esse salubris et vitalis, quia tunc iterum regnat Mars, cuius calore et siccitate membra nascentis roborantur. Unde fetus caloris et siccitatis beneficio egreditur iam robustus. Pour un aperçu de cette doctrine au Moyen Âge, qui ne fait pas la distinction entre les phases embryonnaire et fœtale, voir C. BURNETT, « The Planets and the Development of the Embryo ", in The Human Embryo. Aristotle and the Arabic and European Traditions, DUNSTAN Gordon R. (éd.), Exeter, 1990, p. 95-112. A la différence des autres auteurs, Barthélemy retient l'hypothèse, véhiculée depuis l'Antiquité, que le fœtus peut venir au monde au début du dixième mois de grossesse.

156. DPR VIII.9, 1. 81-94 : Prima facies datur Marti, secunda Soli, tertia Veneri. Prima facies Tauri est Mercurii, secunda Lune, tertia Saturni. Prima Geminorum est Iovis, secunda Martis, tertia Solis. Prima Cancri est Veneris, secunda Mercurii, tertia Lune. Prima facies Leonis est Saturni, secunda Iovis, tertia Martis. Prima Virginis est Solis, secunda Veneris, tertia Mercurii. Prima Libre est Lune, secunda Saturni, tertia Iovis. Prima Scorpionis est Martis, secunda Solis, tertia Veneris. Prima Sagittarii est Mercurii, secunda Lune, tertia Saturni. Prima Capricorni est Iovis, secunda Martis, tertia Solis. Prima Aquarii est Veneris, secunda Mercurii, tertia Lune. Prima Piscium est Saturni, secunda Iovis, tertia Martis. 
157. DPR VIII.9, 1. 61-73 : Nam Sol habet virtutem suam et exaltationem in decimoctavo gradu Arietis et casum in eodem gradu Libre. Venus habet gloriam suam in Piscibus vigesimoctavo gradu, et casum sive tristitiam suam in consimili casu signi oppositi, scilicet Virginis. Mercurius erigitur in Virgine decimoquinto gradu et cadit in Piscibus gradu totidem. Luna erigitur in Tauro tertio gradu et in totidem cadit in Scorpione. Saturnus erigitur in Tauro, vigesimoprimo gradu, et cadit totidem in Ariete. Mars erigitur in Capricorno, gradu vigesimoseptimo et totidem in Cancro cadit. Iupiter erigitur <in decimoquinto gradu Cancri et cadit in Capricorno totidem>.

158. JUSTE, Les Alchandreana primitifs, 2007: 129-135.

159. DPR VIII.20, 1. 22-34 : Est autem hic advertendum quod quandoque Luna coniungitur Soli, Capite vel Cauda existente prope gradum coniunctionis a XII gradibus vel infra. Semper est eclipsis futura maior vel minor, secundum quod Caput vel Cauda magis vel minus accedit ad gradum coniunctionis, et hoc potissime habet locum in eclipsi Solis. Eodem modo de eclipsi Lune hoc tenendum est, quia si Luna convenerit cum Cauda in aliquo signo et Sol fuerit cum Capite in signo opposito, erit eclipsis Lune, et econverso, si Sol fuerit in aliquo signo cum Cauda et Luna in signo opposito cum Capite, erit iterum eclipsis Lune. Et si in eodem gradu convenerit, erit eclipsis generalis. JUSTE, Les Alchandreana primitifs : 463-64, § 24 ; E. Poulle, "Quand le soleil a rendez-vous avec la lune», Il sole e la luna. The Sun and the Moon (Micrologus. Natura, Scienze e Società Medievali), XII, 2004, p. 9-26.

160. Procédant d'une critique systématique des théories précédentes sur la base de la raison et de l'expérience, Grosseteste avance que les comètes sont produites par du feu sublimé, séparé de sa nature terrestre par la virtus celestis qui descend des étoiles fixes ou des planètes et qui attire la comète comme l'aimant attire le fer (S. H. Thomson, " The Text of Grosseteste's De cometis », Isis, 19, 1933, p. 19-25 ; A. C. Crombie, Robert Grosseteste and the Origins of Experimental Science (1100-1700) , Oxford, $\left.1961^{2}: 88-90\right)$.

161. DPR VIII.21, 1. 2-5: Cometa est stella quedam flammis circuita, ut dicit Beda, repente nascens, regni mutationem aut pestilentiam aut bella aut ventos sive estus significans sive pretendens.

162. DPR VIII.11, 1. 2-7: Moventur autem omnes planete duplici motu, scilicet naturali et proprio, scilicet $a b$ occidente in orientem contra motum firmamenti, et motu alieno, scilicet ab oriente in occidentem, et hoc raptu firmamenti, cuius violentia singulis diebus ab ortu ad occasum rapiuntur.

163. DPR VIII.6, 1. 24-31:Tanta autem celeritate spera celi circumfertur, quod nisi adversum eius precipitem cursum planete ei occurrerent et eius impetum moderarent, mundi machina solveretur. Et ideo secundum Alfraganum VII orbes planetarum sunt sub spera se mutuo intersecantes, per quos planete motu consono incedentes raptui firmamenti obviant et eius velocitatem reprimunt et retardant.

164. DPR VIII.2, 1. 78 : Movetur enim a motore infinite potentie, id est a Deo.

165. DPR VIII.2, 1. 109-110 : [...] motus eius singularis et circularis, cuius motor est spiritus qui movet illud sua voluntate.

166. PANTI, Moti, virtù e motori celesti, 2001 : 204-210.

167. DPR VIII.2, 1.145-146: Habet igitur firmamentum [...] omnium tamen motuum velocissimum ; VIII.3, 1. 10-12: [...] hoc modo ad impetus firmamenti moderationem vel ad caloris generati ex eius velocissimo motu repressionem.

168. DPR VIII.22, 1. $45:$ :...] stelle [...] quo ad motum velocissime.

169. DPR VIII.23, 1. 22 : Polus igitur est stella [...] quo ad motum velocissima.

170. DPR VIII.11, 1. 7-10: In motu autem naturali, quo nituntur contra firmamentum, quidam celerius, quidam tardius perficiunt cursum suum, et hoc propter circulorum suorum disparem quantitatem; VIII.11, 1. 197-198: Quanto habent altiores circulos, tanto tardius sunt sui cursus completive.

171. DPR VIII.17, 1.203-217 : Item, cum sit maxime quantitatis et velocissimi motus, sue velocitatis et magnitudinis est occultativus. Cum enim sit octies maior tota terra, ut dicit Macrobius, propter altitudinem sui situs vix bipedalis videtur. Quod autem incomparibiliter maior sit quam apparet patet ex hoc, quod maior videtur in suo ortu et occasu, quando a nobis maxime elongatur, quam in puncto meridiei, quando nobis maxime appropinquat. Unde patet quod in ipsius aspectu decipitur visus noster. Et hoc aperte declaratur in ipsius motu, quia continue movetur velocius quam sagitta, et tamen moveri in aliquo non 
videtur. Pre nimie enim claritatis intensione et excessu, sicut eius quantitas, sic et motus velocitas subterfugit visum nostrum.

172. DPR VIII.15, 1.18-20: Quando Venus est altior Mercurio, tunc est illo segnior motus eius, et econverso, cum est inferior, tunc est celerior, ut dicit Macrobius.

173. DPR VIII.18, 1. 179-181: Et hoc accidit tum propter motus sui velocitatem, tum etiam propter ipsius ad nos vicinitatem, tum propter occultam sibi naturaliter insitam potestatem.

174. Ces notions semblent connues au Moyen Âge depuis la diffusion du commentaire du Timée de Chalcidius, qui ne prendra toutefois une place importante dans la culture astronomique qu'avec le XI ${ }^{\mathrm{e}}$ siècle, et du De nuptiis de Martianus Capella (BАКноUсHE, Les textes latins d'astronomie, 1996 : 255-266 ; EASTWOOD, Ordering the Heavens, $2007: 14$ et 26).

175. DPR VIII.11, l. 127-150 : Primus autem motus planete et proprius fit in circulo qui dicitur ecentricus, sic dictus, eo quod non habeat terram pro centro, sicut zodiacus habet terram pro centro. Unde circulus planete dicitur ecentricus, cuius superficies, cum per centrum terre transeat et terram in duas medietates dividat, centrum tamen suum habet extra centrum terre. Et iste circulus ecentricus est duplex, scilicet deferens et equans. Nam planetis assignantur tres circuli, ut dicit Tholomeus, scilicet equans, deferens et epiciclus. Circulus deferens est circulus planete, per cuius circumferentiam semper movetur epiciclus ipsius planete, dictus ideo deferens, quia defert circulum epicicli. Circulus equans dicitur, super cuius centrum movetur centrum epicicli planete uniformiter, et dicitur equans, quia in illo tenet equaliter planeta cursum suum. Epiciclus etiam est quidam parvus circulus, quem describit planeta motu corporis sui, per cuius circumferentiam movetur corpus planete. Unde in superiori parte sui epicicli movetur ab occidente in orientem, in inferiori vero parte movetur ab oriente versus occidentem. Et ideo patet quod Sol et alii planete in propriis circulis moventur et primis uniformiter, quamvis in diversis diversimode moveantur.

176. Pour les solutions variées apportées par Ptolémée pour expliquer le mouvement de la Lune, de Mercure et des planètes supérieures, voir G. E. R. LLOYD Une histoire de la science grecque, Paris, 1993 : 314-23.

177. VINCENT DE BEAUVAIS, Spec. nat., XV.25: Omnes ergo planete preter Solem, qui progressivus tantum dicitur, dicuntur progressivi, retrogradi, stationarii. Progressivus dicitur planeta, quando movetur ab initio sui epicycli versus finem. Retrogradus vero quando movetur a fine versus principium. Stationarius quando incipit esse progressivus et desinit esse retrogradus vel econverso. Directus etiam dicitur, quando movetur in superiori parte epicycli. Indirectus vero, quando in inferiori.

178. DPR VIII.11, 1. 151-171: In istis itaque circulis diversis triplex motus planetarum est ab astrologis sapientissime deprehensus, motus scilicet directus, stationarius et retrogradus. Directus est, quando movetur directe a principio signi versus finem. Retrogradus vero dicitur, quando econverso tendit a fine signi versus principium. Stationarius autem est, quando stat quasi in medio, ita quod nec principio signi videtur accedere neque fini. Fit autem directio semper in parte epicicli superiori, retrogradatio vero in parte inferiori, statio vero fit in medio. Cuius diversitatis ratio a philosophis diversimode assignatur. Ista tamen retrogradatio solum in $V$ planetis habet locum, non autem in Sole nec in Luna. Non enim Sol neque Luna retrogradiuntur, quamvis in epiciclo moveantur. Et hoc ideo, ut dicunt aliqui, quia radii solares sunt causa istius retrogradationis. Nam virtus radiorum solarium aliquando illos repellit et facit retrogrados, quandoque eos attrahit et sic eos quasi stare cogit, sicut dicit Alfraganus. Alias autem causas assignat Tholomeus, sed hec nunc sufficiant.

179. Ce type de renseignements se trouvait dans l'Histoire naturelle de Pline ou la Mathesis de Firmicus Maternus, mais également dans le De temporum ratione de Bède ou le Liber Alchandrei.

180. DPR VIII.11, 1. 11-25: Nam Saturnus moratur in quolibet signo per XXX menses et complet cursum suum in XXX annis. Iupiter moratur in quolibet per annum unum, et XII annis complet cursum suum. Mars moratur in quolibet signo XLV diebus, et perficit cursum suum in II annis. Sol autem moratur in quolibet $X X X$ diebus et $X$ horis et semisse, et perficit cursum suum in CCC et LXV diebus et quadrante. Mercurius moratur in quolibet signo XXVIII diebus et VI horis, et complet cursum suum in CCCXXXVIII diebus. Venus moratur in quolibet signo XXIX diebus, et complet cursum suum in CCCXLVIII diebus. Luna moratur in 
quolibet signo II diebus et dimidio et VI horis et bisse unius, et complet cursum suum a puncto usque ad punctum in XXXII diebus et VIII horis.

181. Sur ce traité de Pseudo-Ptolémée que Barthélemy a connu par le biais de la compilation d'Arnold de Saxe, voir DRAELANTS, FRUNZEANu, "Sur les traces du De motibus», 2011, et le développement ci-dessus.

182. DPR VIII.14, 1. 30-31: Moratur in quolibet signo LX diebus et XXI horis, et II annis complet cursum suum.

183. DPR VIII. 18, 1. 85-87: Moratur autem in quolibet signo duobus diebus et sex horis et bisse, et in XXVIII diebus complet cursum suum.

184. Pour les valeurs attribuées à la révolution sidérale de la Lune par les auteurs antiques, v. BAKHOUCHE, Les textes latins d'astronomie, 1996 : 188-190, ARMISEN-MARCHETTI, Macrobe. Commentaire au Songe de Scipion : I.6.50-52, t. I : 151-52.

185. DPR VIII.22, 1. 135-142: [...] permutatio temporis non est nisi propter permutationem stellarum in signis diversis super climata VII, ut ex permutatione Lune singulis XXVIII diebus, aut ex permutatione Mercurii et Veneris in omnibus X mensibus vel minus illo, aut ex permutatione Solis in quolibet anno, aut ex permutatione Martis in II annis, aut Iovis in XII aut Saturni in XXX.

186. DPR VIII.22, 1. 135-153: permutatio temporis non est nisi propter permutationem stellarum in signis diversis super climata VII, ut ex [...] aut propter permutationem stellarum fixarum orbis. Permutantur enim in $C$ annis uno gradu, quarum accidit permutatio in omnibus XXXVI milibus annis. Et hoc est annus magnus, quod est ultimum omnium rerum. Hucusque Aristoteles in eodem libro. In libro autem Ciceronis dicit Macrobius: Istius mundani anni finis est, cum stelle omnes omniaque sidera, que aplanes habet, a certo loco ad eundem remeaverint. Hoc autem, ut phisici volunt, post annorum XV milia peracta contingit. 187. Cette confusion apparaît chez plusieurs auteurs, tels Hermann de Carinthie et Alexandre Nequam ; cf. G. de Callataÿ, Annus platonicus, A Study of World Cycles in Greek, Latin and Arabic Sources, Louvain-la-Neuve, 1996 (Publications de l'Institut Orientaliste de Louvain, 47) : 154-159.

188. Sur la relation dialectique entre pensée eschatologique et intérêt pour l'astronomie au Moyen Âge, voir le livre audacieux de J. Fried, Les fruits de l'Apocalypse. Origines de la pensée scientifique moderne au Moyen Âge, Paris, 2004.

189. Timée (35b-36b). Cette théorie est reprise également, entre autres, par Ptolémée dont les traités sont une autre voie de sa diffusion à la fin du Moyen Âge (G. Aujac, Claude Ptolémée, astronome, astrologue, géographe, Paris, 1993 : 83). Selon la tradition pythagoricienne relayée par Macrobe, l'âme est elle aussi constituée d'accords harmoniques, parmi lesquels l'octave, à son tour composée de la quarte et de la quinte (In somn. Scip. I, 6.43).

190. DPR VIII.2, 1. 134-142: Hec autem tria elementa, scilicet aer, ignis et aqua, obediunt cuidam motui celi, sed non uno modo neque uno ordine ipsum imitantur, sed secundum maius et minus, quia quod superius est et levius et purius obedit magis, et quod minus est, tale sequitur tardius et obedit minus. Terra huic celesti virtuti et motui non obedit, ut ab eo suscipiat localem motionem, quamvis ad producendas ex se species diversas suscipiat impressiones.

191. On connaît trois versions latines de ce texte : une anonyme et deux traduites du grec par Guillaume de Moerbeke d'abord et par Pietro d'Abano ensuite (THORNDIKE, «The Three Latin Translations of the Pseudo-Hippocratic Tract on Astrological Medicine", Janus. Revue internationale de l'histoire des sciences, de la médecine, de la pharmacie et de la technique, 49, 1960, p. 104-129; P. KIBRE, "'Astronomia' or 'astrologia' Ypocratis », Studies in Mediaeval Science, Alchemy, Astrology, 1984, p. 133-156; KIBRE, Hippocrates latinus, New York, 1985, VII, p. 94-107; voir aussi les réserves exprimées par W. Vanhamel au sujet du fait que Guillaume de Moerbeke aurait traduit ce texte du grec, «Biobibliographie de Guillaume de Moerbeke », in BRAMS J., VANHAMEL W. (éd.), Guillaume de Moerbeke. Recueil d'études à l'occasion du 700 anniversaire de sa mort, 1286, Leuven, 1989 (Ancient and medieval philosophy. Series 1):370-73). Les passages cités par Barthélemy proviennent de la traduction anonyme que L. Thorndike et P. Kibre pensent avoir été tirée de l'arabe et dont on conserve de nombreux manuscrits dès le XIII ${ }^{\mathrm{e}}$ siècle. Selon Roger 
French, ce texte aurait déjà été connu au XII e siècle FRENCH R., " Astrology in Medical Practice », in Garcia-BAllester L., fRench R., arrizabalaga J., cunningham Andrew (éd.), Practical Medicine from Salerno to the Black Death, Cambridge, 1994, p. 30-59 : 39-42).

192. DPR VIII.18, 1. 193-197 : Et ideo cautus et perfectus medicus secundum doctrinam Ypocratis aspicere debet primam Lunam, et quando est plena luminis, quia tunc crescunt humores in hominibus et medulle, et fit incrementum in mari et in omnibus rebus mundanis.

193. DPR VIII.21, 1. 3-5: Cometa est stella quedam flammis circuita, ut dicit Beda, repente nascens, regni mutationem1 aut pestilentiam aut bella aut ventos sive estus significans sive pretendens.

194. DPR VIII.10, 1. 46 : [...] quando Sol est in Tauro, terra est arabilis et congrua agriculture.

195. DPR VIII.8, 1. 6-8: Dicitur autem lacteus circulus, quia inter omnes circulos celestes notabiliorem habet claritatem. Unde et de nocte navigantes et itinerantes dirigit et deducit ; VIII.22, 1. 61-63 : Stelle [...] Sunt etiam navigantibus prospere et ipsorum viarum in medio maris directive ; VIII.23, 1. 31-33 : Et ideo vocatur stella maris, qui navigantium et scientiam navigalem habentium in mari est directiva .

196. DPR VIII.18, 1.182-211: Unde morborum mutationem medicus perfecte non discernit, qui lunares effectus in corporibus non cognovit. Unde Ypocras in principio Pronosticorum de Luna loquens ait: Est quoddam celeste sidus, in quo oportet medicum providere, cuius providentia est mirabilis et stupenda. Et Galenus in commento De diebus creticis dicit: Rem certam, que non fallit, attendat medicus, quam docuerunt astrologi Egyptiorum, quod per coniunctionem corporis lunaris cum stellis fortunatis fiunt egritudines terminabiles ad bonum, cum contrariis vero contrarie, scilicet ad malum. Et ideo cautus et perfectus medicus secundum doctrinam Ypocratis aspicere debet primam Lunam, et quando est plena luminis, quia tunc crescunt humores in hominibus et medulle, et fit incrementum in mari et in omnibus rebus mundanis. Quando igitur infirmus cadit in lectum, necesse est videre utrum Luna tunc exeat de combustione; tunc enim crescit infirmitas, quousque veniat ad oppositionis gradum, scilicet in plenilunio. Et tunc si fuerit cum malo planeta aut in malo signo et aspexerit dominum domus mortis, qui est signum octavum, id est si aspexerit Martem, qui dicitur dominus signi Scorpionis, tunc de morte timendum est. Si vero fuerit cum bono planeta et in bona domo sive signo et aspexerit dominum domus vite, scilicet Martem, qui est dominus Arietis sive primi signi, de vita tunc sperandum est. Et sic de aliis iudicandum est, ut patet in libro Ypocratis, quem dicitur composuisse de iudiciis infirmitatis secundum Lunam; VIII.28, 1. 5-23 : Ab hac stella dicuntur dies [i.e. canicula] caniculares, in quibus sanguinem minuere vel medicinam laxativam accipere est summe periculosum propter excessum distemperantie aeris in calore. Unde Ypocras in Aphorismis: Ante Canem et post Canem et sub Cane moleste sunt farmacie. Ibi dicitur in Commento quod ante ortum illius stelle et in ortu et post ortum statim periculosum est dare potionem laxativam, quia tunc est aer calidus et siccus, scilicet a calido signo et a calida stella et a Sole tunc in Leone cum Canicula existente. Unde ex modica medicina corpus fieret nimis calidum et siccum, et febris forsitan sequeretur. Medicina etiam evaporaret et minus operaretur, cum corporis pori tunc ex calore aeris sint aperti. Dicit enim ibidem Galenus: Calor extraneus vires extrahit medicine, unde et aqua calida repugnat solutioni, si calor aeris tunc est fortis. Preterea virtus interior tunc est debilis et per potionem vel minutionem plus debilitatur.

197. DPR VIII.1, 1. 180-206 : Et quamvis mundus tot laudabilibus rerum differentiis sit divine virtutis insignitus potentia, multis tamen defectibus et conditionibus miserabilibus quantum ad eius partem inferiorem undique est subiectus. Nam mundus iste, quamvis videatur esse genitor et nutritor corporum, carcer tamen est spirituum et animarum, durissimum est exilium ac locus miseriarum innumerabilium et penarum. Nam mundus est locus reatus et transgressionis, incolatus et peregrinationis, doloris et lacrimationis, laboris et fatigationis, horroris et confusionis, motus et mutationis, fluxus et alterationis, transitus et corruptionis, insolentie et perturbationis, violentie et oppressionis, fraudulentie et deceptionis. In mundo nihil aliud invenitur quam vanitas, malignitas, cupiditas, anxietas, defectibilitas et vetustas. Mundus notos allicit et diligit, ignotos abicit et contemnit. Mundus multis obest, paucis prodest, amatores suos decipit et fallit. Nam multa promittit, sed ad ultimum pauca solvit. Contemptores suos ad modum umbre insequi et apprehendere non desinit, sed suos sequaces fugere satagit et contendit. Et ideo illos, quos maxime divitiis et honoribus hic extollit, in fine maxime depauperare et deicere consuevit. Et, ut verbis 
beati Gregorii utar, fugiendus esset mundus, etiam si nos prosperis demulceret. Qui ergo tot calamitatibus pulsat, quid aliud quam ut deseratur clamat. Sur ce thème, voir R. BULTot, "Cosmologie et 'contemptus mundi' ", Recherches de théologie ancienne et médiévale. Numéro spécial I. "Sapientiae doctrina ». Mélanges de théologie et de littérature médiévales offerts à Dom Hildebrand Bascour O.S.B., 1980, p. 1-23.

198. Dans le Liber de anima, Avicenne réserve un chapitre aux distinctions à faire entre lux, lumen, radiositas, radius: <De luce et lumine>, titre supléé par l'éditrice (Liber de anima seu sextus de naturalibus, VAN RIET S. (éd.), Louvain-Leiden, 1972, pars III, c. 1, p. 169-177).

199. DPR VIII.29, 1. 3-38: De luce autem quid sit secundum rem, utrum scilicet sit substantia vel accidens, diversimode loquuntur auctores. Nam Aristoteles dicit lumen non esse corpus nec a corpore defluxum. Damascenus etiam dicit lumen propriam ypostasim, id est substantiam, non habere. Secundum autem beatum Augustinum super Genesim ad litteram lux est substantia corporea summe simplex in genere corporum, summe multiplex in efficacia, summe mobilis et absolute penetrationis et minime resistentie, et summe disparia et contraria generans et concilians, summe convertibilis, omnis naturalis motionis principium et origo, summe perfectibilis, summe iocunda, summe communicabilis. Unde luce in corporibus nihil est utilius, nihil convenientius, nihil pulcrius, nihil velocius, nihil subtilius, nihil impassibilius, nihil virtuosius invenitur. Est autem differentia inter lucem et lumen. Nam lumen est quidam defluxus a luce sive irradiatio defluens a substantia lucis. Lux autem dicit ipsam fontalem substantiam, super quam lumen innititur. Si enim lux considerata in se esset accidens, necessario per se esset alicui accidens. Accidens autem per se causatur a vera forma subiecti sui. Unde si lux in aere esset accidens, causaretur lux a vera forma aeris, quod esse non potest. Preterea lux mutat subiectum suum. Quod patet, quia lux est naturaliter prius in oriente, consequenter vero in occidente, et lux orientalis generat proximam sibi, et ita deinceps usque ad occidentem. Accidens enim non permutat suum subiectum nec extra illud agit. Ex quo videtur quod lux non sit accidens. Preterea si esset accidens aeris, necessario moveretur aer subito ab oriente in occidentem secundum subitum motum lucis; sic autem moveri subito non convenit aeri nec alicui elemento. Preterea luce nihil est nobilius, accidens autem est ignobilius substantia.

200. Ces deux propriétés lui sont conférées aussi par Bonaventure, (In III Sent., dist. 18, art. 1, q. 1, éd. Opera omnia $1887: 380)$ : Lux propter suam nobilitatem et actualitatem in eodem instanti incipit esse et lucere.

201. DPR VIII.29, 1.138-151: Ex quo manifestum est quod cum lux in genere corporum minimum habeat de materia et maximum de forma, vicinior est forme quam materie. Et ideo ratione materie est in minimo, scilicet in puncto, ratione vero forme est in omni loco. Nam unumquodque habet ita moveri ad locum sicut ad formam suam, ut dicit Aristoteles, quia forma quanto est nobilior, tanto materiam suam plus extendit, ut dicit Algazel. Propter quod forma lucis, quia est nobilissima in genere formarum corporalium, maxime materiam suam distendit. Et ideo unus punctus luminis sive lucis sufficeret ex se ad illuminationem universi orbis propter materie sue nobilitatem et forme summam actualitatem, ut dicit Algazel.

202. De luce, éd. PANTI 2013 : 227, 1.19-21 ; aussi J. P. cUnNINGHAM et M. HOCKNULL, éd., Robert Grosseteste... and Scientific Learning : 8, 22-23, 169-170.

203. DPR VIII.29, 1. 39-87 : Ex quo videtur quod lux non sit accidens, cum aer longe sit ignobilior ipsa luce. Sed si lux est corpus, difficile est intelligere qualiter lux sit in aere vel cum aliquo corpore dyaphano, ut in cristallo, cum duo corpora non possunt simul esse in eodem loco. Non est tamen inconveniens nec impossibile ponere lucem esse substantiam corpoream et esse simul cum alio corpore. Videmus enim aquam et cinerem pariter iungi et misceri, salva utriusque corporeitate et localitate utriusque superficiem ab alio distinguente et utriusque partes infra propriam superficiem continuante. Quantumcumque enim simul misceantur cinis et aqua, permanet tamen aqua in sua corporeitate per partium suarum coherentiam et continuitatem et cinis in sua. Nec propter istam commixtionem et unionem simul sunt duo corpora in uno loco, immo aqua habet suum locum proprium et cinis suum. Sic lux potest esse in aere vel in quocumque alio corpore, salva utriusque corporeitate et suarum partium substantialium continuitate. Unde et lux subintrans ad interiora aeris vel cristalli habet suum locum ipsius superficiem ambientem et a substantia adiuncti sibi corporis distinguentem, quamvis non possit propter simplicitatem sue substantie sensu percipi 
vel videri. Immo quod mirabilius est, multe luces pariter concurrentes impermixte et tamen unite coniunguntur, salva cuiuslibet substantiali forma, qua ab alia differt, licet nulla respectu alterius materialis sit vel formalis, sicut manifeste docet et dicit Dionysius in libro De divinis nominibus. Cuius hec sunt verba: Lumina, inquit, lampadarum cum sint in domo una, tota invicem totis sunt communia, diligentem tamen habent a se invicem discretionem, unita discretione et unitate discreta. Videmus enim multis ad unum lumen accensis lampadibus iuxta omnium lumina unam claritatem indiscrete relucentem, nec poterit quis, ut arbitror, alicuius eorum ab aliis ex aere continente cetera, dum simul sunt ibi, sequestrare aut alterum sine altero cernere, totis inconfuse invicem contemperatis. Subducta autem una simul et proprium lumen recedet, nihil secum ceterorum luminum auferens aut de suo aliquid eis relinquens. Erat enim omnium, ut dictum est, perfecta unitas universaliter commixta et nulla parte confusa, que si tanta est in hoc corporali aere et materiali lumine et cetera. Hucusque Dionysius. In quibus verbis Dionysius manifeste docet luminaria simul uniri, salva tamen cuiuslibet proprietate substantiali et accidentali, quas deferunt secum sive accedendo sive recedendo; VIII.29, 1. 152-165: Una igitur est lux simplex et uniformis in sua essentia, quamvis diversa sint luminaria, que inter se sunt distincta, quamvis videantur in uno actu uniformiter aggregata. Nec unum lumen intrat substantiam alterius, quamvis sensibiliter tamquam unum lumen uniri videantur. Non est ergo inconveniens ponere duo corpora simul esse, quorum unum est subtile et formale et quasi complementum respectu alterius, reliquum autem est materiale et imperfectum. Duo vero corpora glorificata in eodem ubi esse non possunt, quia neutrum respectu alterius materiale potest esse in aliquo vel formale. Sed glorificatum et non glorificatum simul esse possunt sine aliquo inconvenienti, sicut expresse dicit Augustinus.

204. RICHARD FISHACRE, De luce, éd. LONG $2011: 336,1.102-105$ : Nullum mihi est inconveniens multa corpora subtilia vel spiritualia esse in eodem loco et etiam in eodem loco cum corpore grossiori, sicut constat de corpore Christi et aliis corporibus glorificatis quod ianuis clausis intrare possunt.

205. BONAVENTURA, In Sent. IV, dist. XLIX, pars II, sect. II, art. III, quaest. I, éd. Quaracchi 1889, t. IV : 1029 : Ad illud quod obiicitur de corpore glorificato, utrum possit esse simul cum alio; aliqui dixerunt, quod sic, sicut duo radii et lumina simul sunt secundum Dionysium. Sed tamen hoc non credo, quia unum non est materiale respectu alterius nec pertransibile alio; et ideo, quamvis sit ibi subtilitas, deficit tamen penetrabilitas. Nec est simile de luminibus, quia formae sunt habentes multiplicationem non a subiecto, in quo sunt, sed a principio effectivo, sicut dicit Dionysius; et ideo plura corpora gloriosa non possunt esse simul, sicut gloriosum cum non glorioso..

206. DPR VIII.29, 1. 114-128: Lux itaque a summo celo empyreo usque ad centrum mundi est diffusa, que in sua substantia una est et radice et simplex, variata tamen secundum diversitatem recipientium sive Solis sive aliorum corporum superiorum vel inferiorum, ubi est vera substantia, virtus et operatio lucis, licet non luminositatis. Hec est primogenita lux que creata fuit die prima, ut dicit Basilius, que solari corpori et ceteris celestibus quarta die factis superfertur. Unde Sol et cetera sidera sunt primevi luminis vehicula, que propter susceptionem lucis incorporalis sufficiunt sine sue substantie deperditione ad illuminationem perpetuam et perennem, unde ista lux ubique attingit et omnia corpora secundum magis et minus perficit et disponit.

207. DPR VIII.29, 1.111-113 : Presentia lucis necessaria est in omni corpore mixto, per quam contraria in elementatis uniuntur.

208. DPR VIII.2, 1. 157-177 : Est etiam firmamentum mediante motu suo generationis et corruptionis in mundo inferiori principium effectivum. Cuius ratio est, ut dicit Rabanus, quoniam firmamentum sui luminis virtutem generationis effectivam ad terram tamquam ad proprium centrum dirigit et destinat, et radios suos in terre superficie aggregat maxime et coadunat. Sicut enim ostenditur in scientia perspectiva, omne corpus spericum, concavum et luminosum in quolibet puncto sui dirigit unam lineam radiosam in centrum illius corporis luminosi, scilicet ad superficiem illius centri, et quanto quelibet linea radiosa est vicinior alteri linee exeunti ad superficiem centri, tanto ex vicinitate unius ad aliam est fortior ad imprimendum in centro effectum suum. Ex quo manifestum est quod cum corpus celi sit spericum, convexum et luminosum, et terra respectu magnitudinis celi non obtineat nisi vicem puncti, cum sit 
centrum, in terra necessario fit maxima aggregatio celestium radiorum, ex quorum virtute et contactu fit maxima generatio et productio rerum generabilium in terra, que est centrum ipsius firmamenti.

209. De luce, éd. PANTI 2013 : 231-32, l. 103-111 et cUNNINGHAM, HOCKNULL, éd., Robert Grosseteste and ... Scientific Learning : 22-23. Dans le De colore, Grosseteste emploie parfois le mot lux pour désigner la lumière physique incorporée.

210. DPR VIII.29, 1.97-100: Unde virtutem lucis sentit et percipit omnis creatura; invisibiliter enim operatur virtus lucis sensum et motum in animalibus, ut dicitur in libro Fontis vite.

211. DPR VIII.29, 1.101-103: Albumasar in libro De iudiciis astrorum Ypocratem docuisse quod nisi stellarum lux de nocte subtiliaret densitatem aeris, destrueretur omne corpus animatum.

212. P. ex. Richard Fishacre dans sa Questio de luce, éd. LONG 2011 : 337, 1. 114, 1. 117-120 : continue creatur materia lucis, sicut quando creatur anima. [...] Sed in hoc, ut mihi videtur, differt factio lucis et anime quod anima tota, scilicet forma eius et materia creantur, cum creatur anima. Cum autem fit lux, materia tantum creatur; forma vero gignitur a corpore luminoso. Richard Rufus (In II Sent., dist. 13, art. 1, in LONG, NOONE, "Fishacre and Rufus on the Metaphysics of Light: Two Unedited Texts » :544) : lux [...] videtur media esse inter animam et corpus, ipsa adinvicem coniungens. Et est intellectivae partis anime tamquam occasionalis pedisequa; sensitivae vero quasi ministra necessaria; vegetativae vero quasi comes et in coniugium sibi associata.

213. Dans la Summa philosophiae attribuée à Grosseteste, la différenciation entre lux, lumen et splendor est longuement argumentée (Summa phil., tract. 14, c. 5, éd. BAUR 1912 : 534-35).

214. DPR VIII.4, 1. 6-9: Illud enim celum summe est lucidum, influens lucem et splendorem super celum cristallinum sibi propinquum et proximum.

215. DPR VIII.2, 1. 19-25: Celum vero ethereum secundum quosdam vocatur supremum aeris interstitium, quod immediatius spere ignis est coniunctum. Et dicitur ethereum, id est splendidum, quia illud medium inter ignem et aerem confinium ab igne est illuminationis et splendoris receptivum. Etheros enim grece dicitur splendor latine, ut dicit Isidorus; VIII.5, 1. 2-5 : Ether grece splendor dicitur latine. Et vocatur ether secundum Isidorum superior regio supremi interstitii aeris et ignis, ubi est perpetuus splendor luminis sive lucis.

216. DPR VIII.17, 1.45-51: Ideo dicitur splendor Olimpi, quia ut dicit Macrobius in libro Ciceronis, diffinitio fuit Platonis omnium sperarum lumen esse a Sole. Dicit enim Plato in Tymeo: Ingeniavit deus rerum conditor lucem clarissimam, quam Solem vocamus, cuius splendore celum et infra omnia illustrantur, et numerus existeret omnium animantium.

217. Sur cette question, voir A. Vasiliu, Du diaphane. Image, milieu, lumière dans la pensée antique et médiévale, Paris, 1997 (Etudes de philosophie médiévale, 76).

218. DPR VIII.2, 1. 208-211: Unde ex parte forme habet rotunditatem, cum convexitate evexitatem, cum claritate dyaphanitatem sive transparentiam et perspicuitatem, uniformitatem in toto, sed in partibus diversitatem.

219. DPR VIII.2, 1.236-248: Nobilitas itaque celi attenditur in substantie sue simplicitate, puritate, perpetuitate, in forme claritate, dyaphanitate sive perspicuitate et in sperica orbicularitate, in motus uniformitate, velocitate et in virtuositate; in situs sublimitate, quia quo ad situm a centro terre maxime distat, in dimensiva quantitate, quia eius magnitudo imaginationem, immo rationis mensuram superat in potestate, quia omnia inferiora sibi subdita regulat, ordinat et mensurat et, quod super omnia mirabile est, omnia inferiora celum ordinat et immutat, et tamen a nullo inferiori se aliquam immutationem recipit, nec aliquid sibi dissimile in natura et virtute aliqua ipsi celesti corpori se conformat.

220. DPR VIII.29, 1. 40-43: Si lux est corpus, difficile est intelligere qualiter lux sit in aere vel cum aliquo corpore dyaphano, ut in cristallo, cum duo corpora non possunt simul esse in eodem loco ; VIII.31, 1. 4-6 : Lumen est defluxus quidam a substantia lucis in aere sive in alio dyaphano corpore receptus et undique circumfusus; VIII.31, 1. 34 : Lux [...] In materia enim dyaphana obstaculum non invenit ; VIII.32, 1. 8-9 : Radius [...] qui a corpore luminoso exiens et aliquod dyaphanum inveniens non recte procedit.

221. VASILIU, Du diaphane:90-91, n. 138. Elle souligne aussi que la notion de diaphane apparaît ensuite dans la traduction par Guillaume de Moerbeke du De anima de Themistius et le terme 
transparens en lien avec diaphanus dans sa traduction du commentaire d'Alexandre d'Aphrodise sur les Météores d'Aristote. Les deux traductions sont trop tardives pour une utilisation par Barthélemy.

222. D'après le corpus Library of Latin Texts (Brepolis, dir. P. TOMBEUR, corpus en ligne de la latinité patristique et chrétienne médiévale), les contextes liés au diaphane apparaissent dans le milieu franciscain, chez Bonaventure (1217/18-1274), qui n'use qu'une fois du terme dans les Collationes in Hexaemeron, pour qualifier l'eau, tandis qu'on en trouve cinq utilisations chez Jean Pecham (1225/30-1292), dans ses questions sur le De anima et dans ses questions sur la béatitude de l'âme et du corps, pour qualifier un élément et un milieu transparent. Enfin, le mot apparaît plus de cinquante fois chez Ramon Lull (1232/33-1315), en particulier dans l'Arbor scientiae. Chez les dominicains, le terme est présent chez Albert le Grand dès son Commentaire des Sentences, et on le trouve en très grande quantité - plus 170 fois - chez Thomas d'Aquin, principalement dans son commentaire au De anima, II et III (qui contient un développement sur le diaphane et la couleur présentant des similarités avec l'exposé de Barthélemy sur ces sujets), dans son commentaire au De sensu et sensato, mais aussi dans son commentaire aux Sentences (p. ex. dist. 17, q. 1, art. 1) et, avec une signification morale et moins philosophique, dans la Somme contre les Gentils et la Somme théologique, en rapport avec la lumière. Plus tard, Guillaume d'Ockam (c. 1290-1349/50) utilise massivement le terme en lien avec l'exposé sur la couleur, puisqu'on le retrouve une trentaine de fois dans la reportatio de ses Questions sur le livre III des Sentences. Quant aux termes dérivés de celui de transparence, on les trouve plus rarement, mais chez les mêmes auteurs. Evidemment, les résultats des sondages dans ce corpus dépendent des œuvres qui s'y trouvent répertoriées : peu d'œuvres de Bonaventure, et aucune de Robert Grosseteste n'y sont saisies, ce qui déforme nécessairement la perspective. Dans "Le mot et le verre. Une définition médiévale du diaphane", Journal des Savants, 1, 1994, p. 135-162, A. Vasiliu discute des attestations de ces termes chez Robert Grosseteste, Albert le Grand, Thomas d'Aquin, Bonaventure, Jean Buridan, Witelo, etc., en lien avec les traités aristotéliciens De anima, De sensu, De caelo, Metaphysica, Physica, mais aussi avec la Hiérarchie céleste du Pseudo-Denys l'Aréopagite où le mot s'applique au contact lumineux avec la divinité, ce qui n'est pas le cas chez Barthélemy l'Anglais.

223. Sous la graphie diafona, diafonitas I.4,15, éd. SMITH 2001, I : 7, I.4.22-24, aussi p. 9, etc.

224. AVERrò̀s, Commentarium magnum in Aristotelis De anima libros, notamment lib. II.67-76 (418a31-419b45), éd. CRAWFORD 1953 : 230-246; AVERROÈs, Commentarium medium in Aristotelis De generatione et corruptione libros, éd. FOBES, KURLAND 1956, lib. I.24 (159rb40) : 35 ; I.56 (163vb56-59) : 70 ; I.76 (165va54) : 82 ; Compendium libri Aristotelis De sensu et sensato, éd. SHIELDS, BLUMBERG 1949 : 12-18 surtout : $192 \mathrm{rb1}-192 \mathrm{va} 11$.

225. ALBERTUS MAGNus, De homine, tract. I, q. 21, art. 3, part. 1, éd. BORGNET 1896, t. 35 : 187, éd. Anzulewicz, Söder $2008: 165,1.41-43$ : Preterea queritur : quid dicatur ibi perspicuum. Et si dicitur quod perspicuum idem dicitur quod transparens.... Dans les années 1260, Roger Bacon parmi d'autres essaye de délimiter le sens des termes diaphanum, diaphonum, perspicuum, pervium, transparens, translucens, lucidum, dont il dit qu'ils signifient la même chose secundum rem, mais qu'ils diffèrent secundum considerationes aliquas (Tractatus de multiplicatione specierum, in Opus maius, pars II, c. 2).

226. VASILIU, Du diaphane, $1997: 42$.

227. VASILIU, Du diaphane, $1997: 60$.

228. VASILIU, Du diaphane, $1997: 60$.

229. GALLE, "The Dating... of the translatio vetus of Aristotle's De sensu »: 36, cite le Compendium d'Averroès où intervient souvent l'adjectif «diaphane »: diaphanum autem est innatum recipere lucem et perfici ab illa. Et cum diaphanum receperit lucem, fiunt ex hoc colores diversi secundum fortitudinem et debilitatem lucis et paucitatem et multitudinem diaphaneitatis. [...] Necesse est igitur ut color fiat ex admixtione corporis lucidi cum diaphano. Et quia omnia composite sunt ex quatuor elementis et diaphanum ex elementis est aqua et aer et lucidus est solus ignis et hoc est quando fuerit mixtus, ideo 
necesse est ut colores sint compositi ex istis duabus naturis, scilicet natura diaphani et natura luminosi. [...] Et quia hoc manifestum est de colore et color est in superficie corporis terminate, necesse est ut color sit finis diaphani terminate, secundum quod est diaphanum terminatum. Et per hoc distinguitur color a luce: lux enim est perfectio diaphani non terminati.

230. GALLE, « The Dating » : 66-67.

231. Éd. DPR_ $1601: 1143$, c. $7:$ [...] sit extremitas corporis perspicui terminati, vel in eius extremitate, et ibi magis proprie est, eadem natura coloris intus et extra [...] ut dicitur in libro de sensu et sensato capitulo 7. [...] Unde patet quod color perspicui extremitas est, ut dicitur in libro de sensu et sensato capitulo 7 [=De sensu 3, 439a30-b1, b10-12] - et 1172, c. 42 : [...] ut dicitur in libro de sensu et sensato, ubi dicitur : nigredo est privatio albi in perspicuo, sicut amaritudo est privatio dulcedinis in humido [De sensu 4, 442a25-27]. Citations données et comparées avec le De sensu dans GALLE, « The Dating » 67.

232. La controverse demeure au sujet de la chronologie du De colore. Habituellement, on s'accorde pour le placer à la fin de la carrière scolastique de Grosseteste à Oxford, comme une sorte d'aboutissement de son travail sur la lumière (DALES, « Robert Grosseteste's Scientific Works », p. 396-97), mais, de l'avis de Cecilia Panti, il daterait de 1225 (Moti, virtù e motori : 362 ; dans DINKovABRUN et al.. The Dimensions of Colour : 38-43 ; et dans FLOOD, GINTHER et al., Robert Grosseteste and His Intellectual Milieu : 184-190).

233. M. C. SEYMour, Bartholomeus Anglicus and His Encyclopedia: 24-28 et n. 26, exclut à tort l'utilisation du De colore relevée par тномsоN, The Writings of Robert Grosseteste Bishop of Lincoln : 93, qui tire argument de son utilisation par Barthélemy pour dater le De colore de 1220 environ. D'après CROMBIE, Robert Grosseteste and the Origins of Experimental Science (1100-1700): 138, Barthélemy utilise le De colore, sans le nommer, dans le livre III.17, De virtute visibili (où il est question de la multiplication des espèces, de l'émission des species, de la réflexion et de la réfraction), et dans le livre XIX.7 également. Sur l'utilisation du De colore chez Barthélemy et dans sa traduction par Jean de Trévise, voir DINKOVA-BRUN et al.. The Dimensions of Colour : 74-84, et 75 avec la transcription d'après le manuscrit Paris, B.n.F. lat. 16096 conservant le DPR, d'un passage distinct et plus long que celui que nous reproduisons.

234. Est autem color secundum Aristotelem in libro meteororum extremitas perspicui in corpore terminato. Sur cette citation, voir l'abbreviatio d'Oxford au De sensu et sensato, translatio vetus, documentée par GALLE, «The Dating » : 36 : De sensu 3, 439b11-12, tr. vetus : Quare color utique erit perspicui extremitas in corpore determinato [...]. GALLE "The Dating»: 52-53, note que cette définition de la couleur est l'une des citations les plus précoces et les plus populaires du De sensu. On la trouve déjà (isolée) dans le petit traité De potentiis anime et obiectis écrit entre 1228 et 1232 en Angleterre ou à Paris : Unde sua definitio est in libro De sensibus et sensibilibus : 'Color est extremitas perspicui in corpore determinato' (éd. CALlus $1952: 152,1.1-2$ ). On trouve aussi cette définition isolément chez JEAN DE LA ROCHELLE, Tractatus de divisione mutiplici potentiarum animae, composé entre 1233 et 1236, avant sa Summa de anima, éd. MICHAUD-QUANTIN 1964 : 61, 1. 279-280 : color enim est extremitas perspicui in corpore terminato.

235. BAUR, Die philosophischen Werke des Robert Grosseteste : 79-82.

236. Capitulum tertium in quo de natura coloris secundum suam sententiam et etiam secundum differentias et secundum <...> eius ponit multorum recapitulationem. Et alia dicit quod color sit. Color est ultimum perspicui quod est in corporibus. Lux enim secundum se penetrat totum diaffonum materialiter. Non incorporata ei, manet in specie lucis et est perfectio diaffoni non terminati. Secundum vero quod mediate dyaffono admisscitur et incorporatur corpori corpulento inquantum in extremitate ipsius corporis sistitur fluxus sui fortitudo et permanet illustrans corporis determinati superficiem, sic color. Et ideo color est extremitas perspicui secundum quod perspicuum est in extremitate corporis determinati corpulenti, ut materialiter dicatur coloris natura. [...] Ex hiis patet quod in simplicibus non est color. Est enim color per admixtionem corporis luminosi cum dyaffono, ut ignis vel ignei cum reliquis tribus: $\mathrm{ms}$. Oxford, Bodl. Libr., Tanner, 116, f. 87r-111, ici f. 87ra-b, cité par GALLE, « The Dating » : 35-36. 
237. Manuscrits Oxford, Bodl. Libr., Tanner 116, Digby 17, f. 116r-178r, et Oxford, New College 285 , f. 13-27v, d'après GALLE, « The Dating » $: 32$.

238. DPR VIII.9, 1.2/ n. 1: Zodiacus est circulus obliquus in XII partes equales distinctus - Nota de ecclesia et XII apostolorum vita et doctrina

239. DPR VIII.9, 1. 16/ n. 5: Inter ista autem XII signa IV sunt cardinalia - Nota de quatuor Evangelistis et eorum doctrina.

240. DPR VIII.2, 1. 90/ n. 10: Celum [...] semper et uniformiter sicut rota movetur circa axem - Nota quod anima semper movetur circa Christum ; VIII.6, 1.2/ n. 1 : Spera celi, ut dicit Isidorus, est species quedam in rotundum formata - Nota de Christo ; VIII.13, 1.2/ n. 1, et passim: Iupiter, qui secundum errorem gentilium et fictiones poetarum summus deorum fuit pater, planeta est benivolus, calidus et humidus, diurnus, masculinus, in suis qualitatibus temperatus - Nota de Christo et eius conditionibus ; VIII.17, 1. 2/ n. 1, et passim : Sol secundum Isidorum quasi solus lucens dicitur, eo quod sit fons totius luminis - Nota de Christo et de prelato ; VIII.29, 1. 2/ n. 1, et passim : Lux, ut dicit Basilius, est species per omnia sibi similis - Nota de Christo et gratia vel de viro iusto.

241. DPR VIII.10, 1.123/ n. 26, et passim : Virgo est signum terreum, frigidum, mediocre, nocturnum Nota de beata Virgine et eius conditionibus ; VIII.18, 1. 2-4/ n. 1, et passim : Luna dicitur quasi luminum una, id est principalis et maxima, quia corpori solari in magnitudine et pulcritudine est simillima - Nota de beata Virgine, de anima, de ecclesia; VIII.23, 1.2/ n. 1, et passim: Polus, ut dicit Beda, est stella parvissima, a qua tota pars celi superior est sic dicta - Nota de beata Virgine.

242. DPR VIII.17, 1. 55-56/ n. 15 : Sol in medio signiferi incedens bissenos radios emittit ex se - Nota de XII apostolis et XII prophetis ; VIII.22, 1.12/ n. 4 : Secundum Alfraganum stella est lux aggregata in suo orbe Nota de apostolis et aliis, in quibus est gratie plenitudo ; VIII. 29, 1.123/ n. 14 : Unde Sol et cetera sidera sunt primevi luminis vehicula - Nota de angelis et apostolis et religiosis.

243. DPR VIII.24, 1.2/ n. 1 : Arcturus est signum a VII stellis sitis in axe in se revolutis constitutum - Nota de ecclesia et prelatis.

244. DPR VIII. 25, 1. 2/ n. 1 : Orion est astrum tempore hiemali exortum - Nota de martyribus.

245. DPR VIII.26, 1. 7-8/ n. 2 : Ille stelle dicuntur Hyades, id est succule, quia attrahendo humores vel resolvendo eos - Nota de confessoribus et eorum devotione et doctrina.

246. DPR VIII. 27, 1.2/ n. 1 : Pliades sunt VII stelle a pluralitate dicte, que sibi sunt vicine, ab invicem tamen sunt distincte - Nota de differentia et convenientia sanctorum vel virtutum.

247. Le rapprochement entre Canicula et l'Antichrist apparait dans plusieurs textes astrologiques $\mathrm{du}$ XIII ${ }^{\mathrm{e}}$ siècle. C'est le cas par exemple d'un texte de Barthélemy de Parme : alia coniunctio fiet [...] in Lyra, alia in Cigno et alia in Syrio, per quas erit nativitas Antichristi et persecutio fidelium Christianorum per Antichristum (BURNETT, "'Partim de suo et partim de alieno' : Bartholomew of Parma, the Astrological Texts in MS Bernkastel-Kues, Hospitalsbibliothek 209, and Michael Scot ", in BATTISTINI P. et alii (éd.), Seventh Centenary of the Teaching of Astronomy in Bologna, 1297-1997. Actes du colloque de Bologne (1997), Bologna, 2001, p. 37-76 : 66 [21]).

248. DPR VIII.28, 1. 2, n. 1 : Canicula est stella quedam ferventissima - Nota de Antichristo et hereticis.

249. DPR VIII.12, 1.29/ n. 10 : Sub Saturno sunt Capricornus et Aquarius - Nota quales sunt in quibus diabolus regnat ; VIII.13, 1.11/ n. 4 : Hic [Iupiter] bonitate sua reprimit malitiam Saturni - Nota qualiter Christus reprimit vires diaboli.

250. DPR VIII.1, 1. 97-98/ n. 18 : Prima materia [...] a qua omnia materialia incipiunt et in quam redeunt tamquam ad matricem - Nota quod ecclesia est mater omnium electorum.

251. DPR VIII. 1, 1.125/ n. 24 : Nulla enim est tam vilis, tam infima in tota mundi machina pars sive particula- Nota de humilibus.

252. DPR VIII.20, 1. 2-4/ n. 1 : Sunt autem due stelle que non sunt planete, sed tamen naturam et effectum videntur habere planetarum, scilicet Caput Draconis et Cauda - Nota de Antichristo et diabolo.

253. DPR VIII.3, 1.2, n. 1 : Sextum celum est aqueum sive cristallinum - Nota de angelis et de animabus beatis. AUGUSTINUS, De civitate Dei, XI, c. 34, 1. 16 : Hi, qui in nomine aquarum angelos, quae supra caelos sunt, intellegi volunt. 
254. DPR VIII.11, 1.123-126/ n. 17 : Secundus eius motus est quem facit sub zodiaco et habito respectu ad zodiacum, semper in inequali tempore et per inequale spatium transit - Nota de officio delegati vel subdelegati.

255. DPR VIII.9, 1.131/ n. 36 : Domus succedentes sunt mediocris effectus et virtutis - Nota contra ballivos et officiales.

256. Ch. de Miramon, "Spiritualia et Temporalia - Naissance d'un couple », Zeitschrift der SavignyStiftung für Rechtsgeschichte. Kanonistische Abteilung, 123/ 92, 2006, p. 224-287.

257. DPR VIII.17, 1. 46-47/ n. 13 : [...] diffinitio fuit Platonis omnium sperarum lumen esse a Sole - Nota quod a Christo procedunt spiritualia et temporalia.

258. DPR VIII.18, 1.108-109/ n. 24 : Item Luna per interpositionem tumoris terre inter se et Solem defectus sui luminis est passiva - Nota quod abundantia temporalium inducit defectum spiritualium.

259. DPR VIII.19, 1. 20-22/ n. $7:$ Et quanto plus proficit et crescit versus terram, tanto plus decrescit et deficit in lumine versus celum - Nota quod temporalia impediunt vel auferunt eterna.

260. DPR VIII.10, 1.155-157/ n. 34 : Scorpius est secundum Misael signum aqueum, frigidum, fixum et nocturnum, domus prima Martis, nullius exaltatio - Nota de malis mundanorum ; VIII.18, 1. 24/ n. 5 : Quando Soli opponitur [Luna], tota ad terram et nihil ad celum illuminatur - Nota contra gloriam mundanam ; VIII.19, 1. 12/ n. 4 : Luna etiam, quando incidit in umbram terre, perdit lumen suum - Nota contra amorem mundanorum ; VIII.21, 1. 13/ n. 3 : Brevi spatio solent apparere, scilicet per VII dies - Nota contra brevitatem glorie mundane; VIII.33, 1.15/ n. 3 : Ex quo patet quod cum Sol sit maior terra, ipsa terra facit umbram conoidem - Nota de amore mundanorum veniali.

261. DPR VIII.14, 1. 26-27/ n. 5 : Secundum Tholomeum etiam sub Marte sunt hec signa Scorpius et Aries, et sunt domus eius; regnat in Capricorno - Nota de ballivis potentum, qui sunt scorpiones per dolositatem, arietes per cervicositatem et capricorni per voluptatem.

262. DPR VIII.20, 1.25/ n. 4 : Nota quod maior tribulatio erit, prout coniunctio Christi et ecclesie plus appropinquabit; VIII.22, 1. 155/ n. 43 : nota quod ultimus finis omnibus est incertus.

263. DPR VIII.18, 1. 98/ n. 22 : Nota quod oppositio spiritualis potestatis contra regalem vel econverso est causa depauperationis ecclesie et clericorum.

264. DPR VIII.22, 1. 79/ n. 26 : Nota quod familiaritas parit contemptum; VIII.22, 1.96/ n. 30 : nota de illo qui habet trabem in oculo proprio et videt festucam in alieno.

265. DPR VIII.2, 1.97/ n. 11 : Nota quod una est anima rationalis, quamvis plures habeat rationes, qui pointe vers une des définitions de l'âme données par Aristote dans le De anima.

266. Sur l'authenticité du traité voir B. DISTELBRINK, Bonaventurae scripta authentica, dubia vel spuria critice recensita, Roma, 1975 [171].

267. BONAVentura, De ecclesiastica hierarchia, IV.3, éd. PELTIER 1866, t. VII : 476 : Coelorum nomine auctoritas judicum et praelatorum designatur ; IV.4, éd. : 480 : Coelorum nomine religiosi et contemplativi significantur; Coelorum nomine doctores sacrae scripturae significantur; IV.6, éd. : 489 : Firmamenti nomine Christus significatur; IV.7, éd.: 493 : Coeli natura et proprietas quomodo B. Virgini Mariae conveniant.

268. Voir MEYER, Die Enzyklopädie des Bartholomäus Anglicus, et l'introduction de I. VENTURA à l'édition du livre XVII du DPR (éd. VENTURA 2007).

269. Cette compilation de contenu médical emprunte une bonne partie de sa matière au DPR. Cf. mss Bâle, Universitätsbibl. D.II.13, Sevilla, Bibl. Capitular y Colombina 7-4-23 et édition à Mainz, 1531.

270. Cf. B. VAN DEN ABEELE, «Moralisierte Enzyklopädien in der Nachfolge von Bartholomäus Anglicus : das 'Multifarium' in Wolfenbüttel und der 'Liber de exemplis et similitudinibus rerum' des Johannes de Sancto Geminiano ", in MEIER, Die Enzyklopädie im Wandel : p. 279-304, et I. VENTURA, «Die moralisierten Enzyklopädien des späteren Mittelalters: ein Überblick unter Berücksichtigung der Fallbeispiele des Lumen Anime, des Liber de exemplis et similitudinibus rerum und des Liber Similitudinum Naturalium ", Reti medievali, 4, janvier 2003 (http:// 
www.storia.unifi.it/_RM/rivista). Dans la partie consacrée au monde végétal par Domenico Bandini dans son Fons memorabilium, I. Ventura a identifié l'apport important du livre XVII du DPR. Ce n'est pas le cas pour les deux livres où Bandini traite des réalités célestes (lib. VII et VIII), pour lesquels Bandini ne cite qu'à de rares occasions le texte de Barthélemy (nous avons repéré une seule occurrence: Oxford, Balliol College 238B, p.105rb, ms. consulté sur le site Early Manuscripts at Oxford University, URL http://image.ox.ac.uk/ . Sur Bandini, voir, avec précautions, M. SCHÜRER, Die Enzyklopädie der berühmten Männer und Frauen. Domenico Bandini, sein „Fons memorabilium universi“ und die kompilatorische Biographik der Renaissance, Tübingen, 2017 (Spätmittelalter, Humanismus, Reformation, 97) et H. MEYER, Das Enzyklopädiekonzept des 'Fons memorabilium universi' des Domenico Bandini im Verhältnis zur Tradition, in Frühmittelalterliche Studien , 27 (1993), p. 220-240.

271. Coeliloquium morale, éd. Bologna 1529. Le dossier trinitaire du DPR est déployé au livre I, ch. $6-17$, f. $8 \mathrm{v}-14 \mathrm{r}$, alors que les chapitres du livre VIII du DPR sont parsemés partout dans le reste de l'ouvrage. Ainsi, les lignes réservées au monde et au ciel empyrée figurent au livre II, ch. 1-2, f. 20r-23r, les descriptions des signes zodiacaux sont abondamment moralisées dans le livre VI, ch. 1-39, f. $74 \mathrm{v}-116 \mathrm{v}$, etc.

272. Bonaventura, Sermones de sanctis, éd. Peltier 1868, t. 14, sermo I (p. 1b, 3a), sermo II (p. 5b, $6 a)$.

273. S. WENZEL, Latin Sermon Collections from Later Medieval England, Cambridge, 2005 : 319.

274. M. J. MORRIN, John Waldeby, O.S.A., c. 1315-c. 1372. English Augustinian Preacher and Writer, with a Critical Edition of His Tract on the "Ave Maria», Roma, 1975 : Stella maris est polus articus que secundum Bartholomeum [...] in mare est directiva (p.90). Waldeby est entré chez les chanoines augustins de Lincoln vers 1334. Devenu docteur en théologie en 1354, on le trouve ensuite au couvent des augustins de York dont la bibliothèque possédait un exemplaire du DPR.

275. Sermones de sanctis, éd. Nuremberg 1487 : aaiiii. Il s'agit d'une collection de sermons connue sous le titre Thesaurus novus.

276. M. ANDERSSON-SCHMitT, H. HALlBerG, M. HEDLUnd, Mittelalterliche Handschriften der Universitätsbibliothek Uppsala, Bd. 3, C201-300, Stockholm, 1990 : 313-323, ms. C295, f. 4.

277. Rosarium sermonum, pars secunda, sermo XXXIII, éd. Venezia 1498 : 326vb-327vb.

278. Voir le contenu de ce passage plus haut $n .197$.

279. Stimulus conscientiae, II, v. 1162-93, éd. MORRIS 1863.

280. Gerardi Magni. Epistolae, éd. MULDER 1933 : 272 et G. ÉPINEY-BURGARD, Gérard Grote, fondateur de la Dévotion Moderne : Lettres et traités, Turnhout, 1998.

281. M. ANDERSSON-SCHMITT, H. HALLBERG, M. HEDLUND, Mittelalterliche Handschriften der Universitätsbibliothek Uppsala, Bd. 2, C 51-200, Stockholm, 1989: 20-24, ms. C59, f. 154v, où le passage est attribué à Albertus De proprietatibus rerum. Ms signalé par MEYER, Die Enzyklopädie des Bartholomäus Anglicus, $2000: n^{\circ} \mathrm{A} 26$.

282. JOHANNES VON PALTZ, Opuscula, éd. BURGER 1989, t. III : 111, 1. 21-28.

283. Catalogus gloriae mundi, Lyon, 1529, pars XII, consid. 5, f. 6 rb.

284. Hierologia sive de sacro sermone, continens summam atque compendium positivae theologiae, lib. II, Lyon, 1617 : 82-96.

285. Mansiones festaque Hebraeorum, Barcelone, 1725 (p. ex. propriétés de Vénus et d'Orion, p. 69 et 122); Mansiones encomiasticae adaptatae quadraginta duobus iuxta Evangelium Matthaei progenitoribus Christi Domini, Madrid, 1737 (p. ex. prima pars : propriétés du Soleil :229; secunda pars : étymologie de Saturne et propriétés du signe du Verseau : 733 et 855); Mansiones morales, Madrid, 1737 (p. ex. propriétés du Soleil : 77) ; Mansiones mysticae et asceticae ad fratres, et moniales, Madrid, 1737 (p. ex. propriétés d'Arcturus : 575).

286. Nous avons consulté le manuscrit Paris, B.n.F. lat. 7357. Les éléments anatomiques repris par Savonarole correspondent généralement aux résumés récapitulatifs que Barthélemy insère par la 
formule Recollige (p. ex. au sujet du nez f. $29 \mathrm{vb}=D P R$ V.13, des lèvres f. $35 \mathrm{vb}=$ DPR V.17) ; les données astrologiques se retrouvent dans la section qui occupe les f. 64r-66v.

287. M. BINDSCHEDLER, Der Lateinische Kommentar zum Granum sinapis, Basel, 1949 : 106-112.

288. Annibale Rosselli (1525-1592), Pymander Mercurii Trismegisti, Cracovie, 1584 : 10 (IV, De coelo, comm. III, quaest. I) ; M. MUcCILLO, « Der ,scholastische' Hermetismus des Annibale Rosselli und die Trinitatslehre », in MULSow M., éd., Das Ende des Hermetismus, Tübingen, 2002, p. 61-101.

289. R. Goclenius (1547-1628), Lexikon Philosophicum (...), Frankfurt, 1613:654-655 ; M. Wolfes, «Goclenius, Rudolph » in Biographisch-Bibliographisches Kirchenlexikon, t. 18I, 2001 : 514-519; voir aussi la notice à son nom sur le site Scholasticon (disponible à l'URL : http://www.scholasticon.fr/ index_fr.php) s. dir. Jacob Schmutz.

290. Ed. NANNUCCI 1846, où l'on trouve des références au mouvement des planètes (DPR VIII.6, 1. 24-29 = Paradiso, X, p. 620) et la description de Saturne (DPR VIII.12 = Paradiso, XXI, p. 685).

291. Ed. LACAITA 1887, vol. IV, p. ex. au sujet des phénomènes météorologiques (DPR VIII.17, 1. 180-183 = Purgatorio, XXI, p. 8).

292. Sur Bernard de Lavinheta, voir A. LLINARÈs, « Le lullisme de Lefèvre d'Etaples et de ses amis humanistes ", in L'humanisme français au début de la Renaissance, Paris, 1973, p. 127-135 ; M. PEREIRA , «Bernardo Lavinheta e la diffusione del lullismo a Parigi nei primi anni del '500 ", in Interpres. Rivista di studi quattrocenteschi, 5, 1983/1984, p. 242-65.

293. Practica compendiosa artis Raymundi Lullii, Lyon, 1523. L'ouvrage figure parmi les livres interdits à Parme en 1580 (Index des livres interdits. T. 9 : Index de Rome 1590, 1593, 1596, avec étude des index de Parme, 1580 et Munich, $\left.1582: 91, \mathrm{n}^{\circ} 65\right)$. Il sera toutefois réédité à Cologne en 1612 par un autre partisan du lullisme, le calviniste Johann Heinrich Alsted.

294. Practica compendiosa, f. 100r.

295. Sans dissocier l'astronomie de l'astrologie, Lavinheta attribue un sens large à la science des astres pour y inclure des notions de comput et un calendrier (Practica compendiosa, f. 128r-145r).

296. Toutes ces occurrences, qui ne sont jamais associées au nom de Barthélemy l'Anglais, figurent aux f. 131v-133r, 136v-137r.

297. Gearoid Mac Niocaill a édité le texte sur la base des manuscrits Dublin, Trinity College, ms. 1299 et Royal Irish Academy, ms. 23 Q10, disponibles sur le site Irish Script on Screen : http:// www.isos.dias.ie/ («Bartholomaei Anglici de proprietatibus rerum liber octavus. Leagan Gaeilge o thus na $15 \mathrm{u}$ aoise », Celtica, 8, 1968, p. 201-242 ; 9, 1971, p. 266-314).

298. Il s'agit du manuscrit München, B.S.B., Clm 28846, ancienne cote Nat. Museum 3631, f. $115 \mathrm{v}-120 \mathrm{v}$. La description des manuscrits de cette série de cotes est en cours par Brigitte Gullath. En attendant, voir la notice de P. LEHMANN, Mittelalterliche Handschriften des K. B. Nationalmuseums zu München, München, 1916 (Sitzungsberichte der Königl. Bayerischen Akademie der Wissenschaften, philos.-philol. und hist. Klasse, Jg. 1916, 4. Abhandlung) : 55-58. Le manuscrit contient surtout du matériel de prédication, des traités de pénitence, de confession. Il est disponible dans la Digitale Bibliothek de la Bayerische Staatsbibliothek: http://www.digitalesammlungen.de.

299. Chantilly. Le Cabinet des manuscrits, Paris, 1900, t. I : 258-60 (la description du Catalogue général des manuscrits des bibliothèques publiques de France est disponible sur le site http:// www.calames.abes.fr. Nous remercions D. Juste de nous avoir signalé ce manuscrit.

300. À toutes fins utiles, nous indiquons pour chaque extrait la page de l'édition du DPR publiée à Francfort en 1601 (sigle DPR_1601).

301. Nous n'avons pas vu ce manuscrit. Toutefois, les différentes phrases transcrites par W. Schum, Beschreibendes Verzeichnis der Amplonianischen Handschriften-Sammlung zu Erfurt, Berlin, $1887: 242$ pour les items allant du f. $5 \mathrm{v}$ au f. $18 \mathrm{v}$ se retrouvent toutes dans le DPR. 
302. Pour une description de l'ensemble du manuscrit, voir Catalogus codicum manuscriptorum medii aevi latinorum qui in bibliotheca Jagellonica Cracoviae asservantur, 1980, vol. I : 268-275. Nous remercions chaleureusement $\mathrm{Sl}$. Szyller pour nous avoir procuré la copie de ces feuillets.

303. JUSTE, CCAL, I, Les manuscrits astrologiques : 81-83. Manuscrit consulté sur le site de la Digitale Bibliothek de la Bayerische Staatsbibliothek : http://www.digitale-sammlungen.de/.

304. Le manuscrit Oxford, Bodl. Library, Digby 58, f. 10-34, a le même incipit, mais nous n'avons pas eu la possibilité de le consulter.

305. Thorndike-Kibre, col. 1714.7. JUSTE, Les Alchandreana primitifs : 352) présente ce manuscrit pour les extraits des Alchandreana qu'il contient.

306. J. TRUHLÁ $\check{R}$, Catalogus codicum manu scriptorum latinorum qui in C. R. Bibliotheca publica atque Universitatis Pragensis asservantur, Prague, 1905, I: 117-119. Manuscrit consulté sur le site Manuscriptorium de l'European Digital Library : http://www.manuscriptorium.com.

307. N. R. KER, A. J. PIPER, Medieval Manuscripts in British Libraries, Oxford, 1992, vol. IV : 266-269.

308. I. VALENTINELLI, Bibliotheca manuscripta ad S. Marci Venetiarum, Venezia, 1868, t. IV : 262-263, classis XI, cod. 86. Voir aussi L. Thorndike, «Notes upon some Medieval astronomical... manuscripts » : 46 qui, en signalant le manuscrit, mais sans remarquer qu'il s'agit d'excerpta du DPR, rapprochait les graphies Misael et Miscalath de Messahala. Le manuscrit est mentionné également par P. O. Kristeller, Iter italicum, 1967, vol. II : 228, qui n'indexe toutefois pas le texte des f. 1r-11r. Nous tenons à exprimer notre gratitude à M. Paulmier-Foucart d'avoir collationné pour nous ce manuscrit.

309. Le texte du Multifarium compilé à partir du De proprietatibus rerum a le même incipit. Conservé dans un seul manuscrit (Wolfenbüttel, Herzog-August Bibl. 200, Gud. lat.4), le Multifarium débute avec des allégories concernant les réalités célestes (cf. B. Van den Abeele, «Moralisierte Enzyklopädien in der Nachfolge von Bartholomäus Anglicus: das „Multifarium“... »).

310. Manuscrit consulté sur le site de la Bibliothèque virtuelle des manuscrits en Suisse : http:// www.e-codices.unifr.ch/fr, où l'on trouve aussi sa description.

311. R. SCHIPKE, Die mittelalterlichen Handschriften der Ratsschulbibliothek Zwickau, Berlin, 1990 : 93-94. Le texte a le même incipit que le manuscrit Oxford, Lincoln College, 57, que signale déjà MEYER, Die Enzyklopädie des Bartholomäus Anglicus, 2000 :176.

\section{RÉSUMÉS}

Le livre VIII de l'encyclopédie naturelle de Barthélemy l'Anglais, intitulé De mundo et corporibus celestibus, porte sur la cosmologie, l'astrologie et l'astronomie (le texte critique, établi depuis 2012, est en cours de parution dans la collection De diversis artibus chez Brepols). Les sources explicites et implicites en sont ici identifiées dans le détail. Certaines montrent l'influence de traités rédigés au début $d u$ XIII ${ }^{\mathrm{e}} \mathrm{s}$. entre la France et l'Angleterre et l'usage de compilations intermédiaires élaborées à la même époque, d'où proviennent $\mathrm{p}$. ex. certaines références à Isidore de Séville, à Ptolémée ou Abû Ma'shar. Parmi les sources explicites, on trouve Raban Maur, Michel Scot, Zael (Misael); parmi les implicites, l'Experimentator (compilation encyclopédique se référant souvent à Isidore et Ptolémée), et des auteurs contemporains comme Robert Grosseteste, Alexandre de Halès, Arnold de Saxe (à travers lequel sont cités les Iudicia Ptolomei), mais on note aussi une influence de Richard Fishacre et Richard Rufus. La deuxième 
partie de l'article étudie les théories cosmologiques et astrologiques décelables dans ce livre, et met en lumière de nombreuses erreurs et approximations qu'il comporte. Si la transmission du texte peut en être la cause, il est aussi probable que Barthélemy, davantage intéressé par le potentiel d'allégorisation de ces informations, ne soit pas lui-même expert en matière cosmologique et ait puisé ses informations à d'autres compilations qu'il comprenait mal. Néanmoins, son texte se révèle être un témoin intéressant des efforts de synthèse du savoir entrepris dans les années 1250 et sa réception abondante tout au long du Moyen Âge relativise les constats d'incohérence et de méconnaissance.

The title of Book VIII of Bartholomeus Anglicus' natural encyclopedia is 'De mundo et corporibus celestibus'; it deals with cosmology, astrology and astronomy. The critical edition, achieved since 2012, is being published at Brepols in the 'De diversis artibus' series (Draelants-FrunzeanuVentura). This article discusses in detail each explicit and implicit sources of Book VIII. Some of them are showing the influence of treatises written in north-west France or southern England in the beginning of the thirteen century and some show the use of intermediary compilations of the same period; some references to Isidore of Seville, Ptolemy or Abu Ma'Shar are coming from them. Among the explicit sources, one finds Hrabanus Maurus, Michael Scot, Zael ("Misael"); among the implicit sources, the so-called Experimentator (an encyclopedic compilation often referring to Isidore and Ptolemy), and some contemporary scholars such as Robert Grosseteste, Alexander of Hales, Arnoldus Saxo (who quotes the Iudicia Ptolemei used by Bartholomeus). There is also an influence of Richard Fishacre and Richard Rufus. The second part of the article looks at the cosmological and astrological theories of Book VIII and highlights numerous mistakes and approximations. These may be due to the transmission of the text, but it may also be possible that Bartholomeus, not an expert in cosmology, was more interested by the potential allegorisation of these data and founded them in other compilation, without fully understanding them. Nevertheless, his text remains an interesting testimony of the effort made in the years 1250 to gather a specific knowledge. Its afterlife throughout the Middle Ages put into perspective or counteract the findings of inconsistencies or ignorance.

\section{INDEX}

Keywords : Bartholomeus Anglicus, De proprietatibus rerum, Medieval Encyclopaedia, Michael Scot, Experimentator, Astronomy, Cosmology, Manuscripts

Mots-clés : Barthélemy l'Anglais, De proprietatibus rerum, Medieval Encyclopaedia, Michel Scot, experimentator, astronomie, cosmologie, manuscrits

\section{AUTEURS}

\section{ISABELLE DRAELANTS}

Isabelle Draelants, docteur en histoire (Université catholique de Louvain) et habilitée à diriger des recherches (Université Paris IV Sorbonne), est directrice de recherche au CNRS. Ses recherches portent sur la transmission des connaissances en sciences naturelles au Moyen Âge. Elle porte le projet SOURCENCYME de corpus des encyclopédies médiévales, dont l'objectif est l'identification des sources latines, gréco- et arabo-latines véhiculées par les encyclopédies médiévales du XIII ${ }^{\mathrm{e}} \mathrm{s}$. (sourcencyme.irht.cnrs.fr). 


\section{EDUARD FRUNZEANU}

Eduard Frunzeanu, docteur en histoire de l'Université de Montréal depuis 2007, s'intéresse à l'histoire intellectuelle et à la transmission des textes au Moyen Âge. Il a collaboré au projet SOURCENCYME et travaille à présent pour l'EquipEx Biblissima (Bibliotheca bibliothecarum). 